\title{
Auflösungsexperimente von Kaolinit, Montmorillonit, Illit, Serizit und Talk in Batch- und Durchfluß-Reaktoren
}

\author{
Dissertation \\ zur Erlangung des Doktorgrades \\ der Mathematisch-Naturwissenschaftlichen Fakultäten \\ der Georg-August-Universität zu Göttingen
}

vorgelegt von

Sabine Schlabach

aus Göttingen

Göttingen 2000 
D 7

Referent:

Prof. Dr. H. Heinrichs

Korreferent:

PD Dr. M. Dietzel

Tag der mündlichen Prüfung: 31. Oktober 2000 


\section{$1 \quad$ Einleitung}

1.1 Bedeutung der chemischen Verwitterung ....................................

1.2 Der Boden als Reaktionskörper .............................................

1.3 Experimentelle Untersuchungen und natürliche Reaktionsmechanismen ............2

$1.4 \quad$ Experimenteller Ansatz ........................................................

2 Theoretische Grundlagen

2.1 Allgemeines...............................................................

2.2 Bestimmung von Auflösungsraten aus Lösungs-Experimenten.......................7

2.3 Ermittlung weiterer Kenngrößen aus den Experimenten ........................9

2.4 Fehlerbetrachtung der berechneten Kenngrößen ...............................9

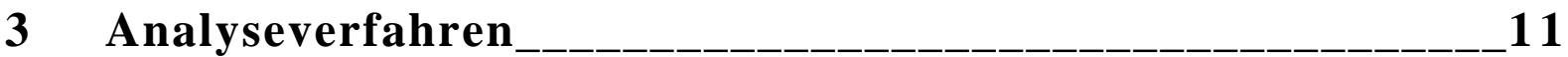

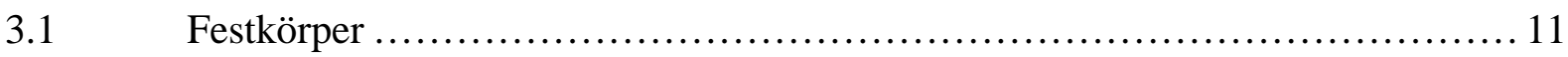

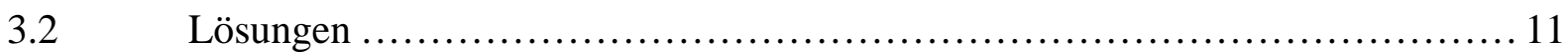

Fehlerbetrachtung .......................................................... 12

4 Experimenteller Aufbau _-_-_-_-_-_---_-_-_-_-_---_-_-_14

4.1 Zusammenstellung verschiedener experimenteller Methoden........................ 14

4.2 Beschreibung der verwendeten experimentellen Methoden..................... 16

4.2.1 Geschlossene Systeme - Batch-Experimente ............................. 16

4.2.2 Offene Systeme - Durchfluß-Experimente ............................... 17

5 Probenmaterial - 20

5.1 Mineralpulver......................................................... 20

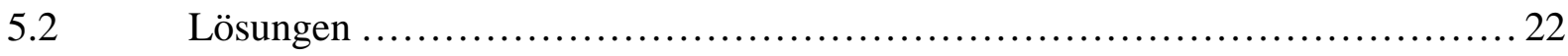

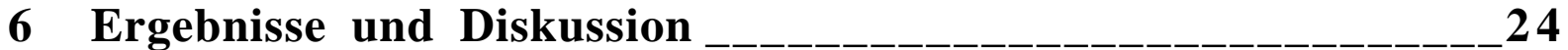

6.1 Durchgeführte Experimente........................................... 24

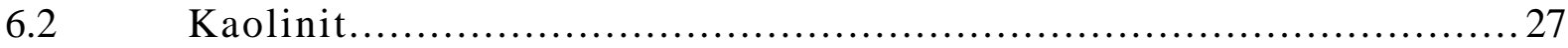

$6.3 \quad$ Montmorillonit ........................................................ 42

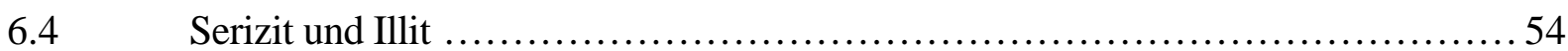

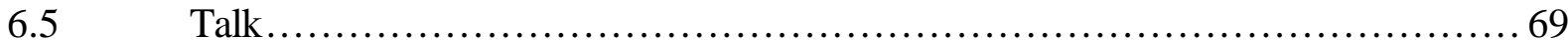

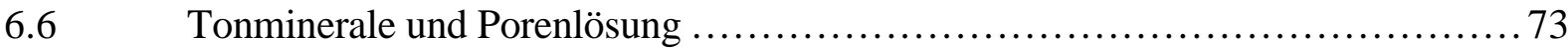

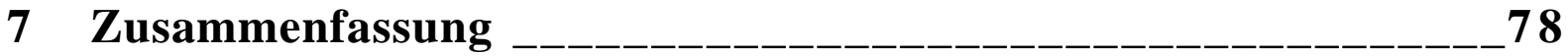

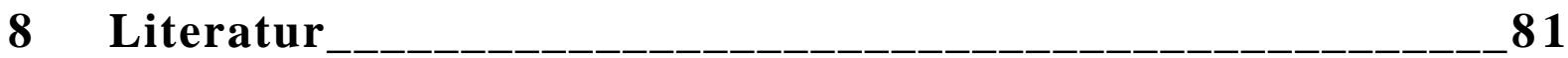


9 Anhang _-_-_-_-_-_-_-_-_-_-_-_-_-_-_-_-_-_-_-_-_-_ 1

9.1 Abbildungs- und Tabellenverzeichnis des Anhangs ..........................

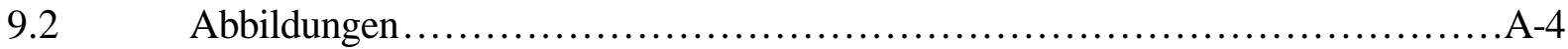

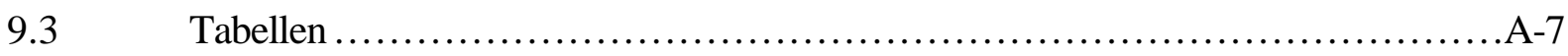

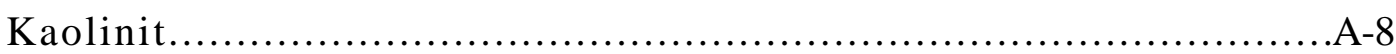

Montmorillonit ................................................. A-25

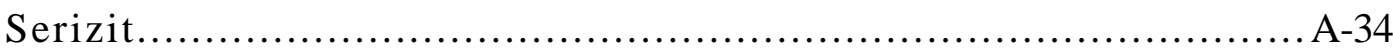

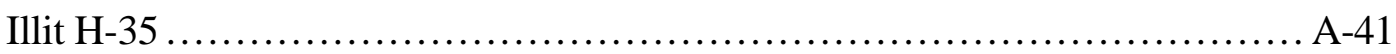

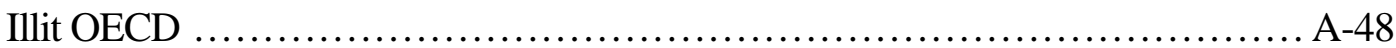

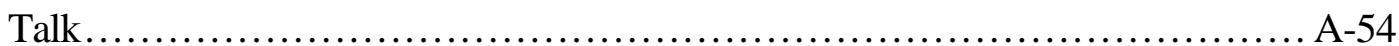




\section{$1 \quad$ Einleitung}

Die vorliegende Arbeit entstand im Teilprojekt „Wechselwirkungen zwischen Lösungen und Festkörpern in offenen Systemen und strömenden Medien" im Rahmen des von der Deutschen Forschungsgemeinschaft unter der Kurzbezeichnung „SFB468” geförderten Sonderforschungsbereiches „Wechselwirkungen an geologischen Grenzflächen”.

\subsection{Bedeutung der chemischen Verwitterung}

Wechselwirkungen zwischen Festkörpern und Lösungen spielen in geologischen Prozessen eine wichtige Rolle. Die chemische Verwitterung kontrolliert maßgeblich die Hydrogeochemie der Gewässer. Wasser tritt sowohl als Reaktant als auch als Transportmedium für die gelösten oder partikulären Komponenten auf. Die Geschwindigkeit der Verwitterung hat einen entscheidenden Einfluß auf die Entwicklung der Böden und die Verteilung von Nährstoffen in Böden. Hinzu kommt noch ein wesentlicher Einfluß auf die Zusammensetzung und Qualität des Grundwassers. So liegen Böden als Puffer zwischen Atmosphäre und Grundwasser, indem sie eingetragene Säuren teilweise neutralisieren können. Bedeutende Protonenquellen hierbei sind durch anthropogene Verunreinigungen in die Atmosphäre eingebrachte Schwefel- und Stickoxide, die in Verbindung mit Wasser zu Schwefelsäure, schwefeliger Säure und Salpetersäure reagieren. Nach Pleßow et al. (1997) beträgt der allein auf Basis der S- und NOxide basierende jährliche Eintrag von $\mathrm{H}^{+}$in der Bundesrepublik Deutschland etwa 3,5 kg/ha. Die Hauptmenge der atmosphärisch transportierten Säurebildner wird zunächst trocken deponiert und schließlich vom Regen abgewaschen. Mit dem Sickerwasser erfolgt dann der Weitertransport in die Böden.

Mit zunehmender Versauerung der Böden und somit auch der Bodenwässer steigen die Gehalte von ,umweltrelevanten” Elementen in den Bodenwässern an (Kuntze \& Herms 1986). Bei tiefgründiger Versauerung der Böden, wie sie bereits in einigen Gebieten Deutschlands (z.B. Solling und Sösegebiet) beobachtet wurde (Deutschmann 1994, Malessa \& Andreae 1990, Malessa 1993), sind schließlich auch die Grundwässer und damit verbunden die Trinkwässer gefährdet. So sind in Einzugsgebieten von Talsperren im Harz, einem wichtigen Trinkwasserreservoir, bereits einige Quellen und Zuflüsse zeitweise oder dauernd versauert und zeigen erhöhte Konzentrationen toxischer Elemente (Frei et al. 2000, Heinrichs et al. 1986, Matschullat et al. 1990). Die freigesetzten Elemente stammen dabei nicht nur aus dem natürlichen Stoffkreislauf, vielmehr werden auch die über viele Jahrzehnte in den oberen Bodenschichten akkumulierten, anthropogenen Spurenstoffe durch die Versauerung mobilisiert. Besonders zu berücksichtigen sind hierbei die verschiedenen Schwermetalle, die häufig schon in geringen Mengen schädlich sein können. 


\subsection{Der Boden als Reaktionskörper}

Der Feinbodenanteil $(\varnothing<2 \mathrm{~mm})$ des Bodenkörpers besteht aus Mineralen, organischen Komponenten und röntgenamorphen Si/Al-Fe-Phasen. In den silikatischen Böden Mitteleuropas sind als kristalline Phasen vor allem Quarz und Feldspat sowie die Schichtsilikate Illit, Chlorit, Kaolinit und verschiedene Wechsellagerungsminerale von Bedeutung (Scheffer \& Schachtschabel 1998, Sposito 1998).

Bei der Verwitterung der silikatischen Bodenminerale werden Alkalien und Erdalkalien freigesetzt und $\mathrm{H}^{+}$-Ionen verbraucht. Die Lösungsgeschwindigkeit der Minerale reicht jedoch aufgrund des geringen Umsatzes nicht aus, um die eingetragenen Säuren vollständig zu neutralisieren. Als Folge dieser Reaktionsträgheit sind in vielen Gebieten die Versauerungsfronten bereits in größere Tiefen verlagert. Besonders betroffen sind dabei Standorte, die aus basenarmen Gesteinen aufgebaut sind (van Breemen et al. 1984, Reuss et al. 1987, Schindler 1988).

In Mitteleuropa werden überwiegend saure bis neutrale Lösungen als Niederschläge in die Böden eingetragen. Die Verwitterung ist somit protonenkontrolliert und stellt eine Neutralisationsreaktion dar. Zur Beurteilung des Neutralisationsvermögens ist es wichtig, die geschwindigkeits-bestimmenden Parameter der ablaufenden Reaktionen zu kennen. Außerdem spielt in der Natur auch die ligandenkontrollierte Auflösung eine bedeutende Rolle. Lieferant der Liganden sind vor allem verschiedene organische Säuren. In den Boden werden sie durch Zersetzung organischen Materials oder direkt über die Pflanzenwurzeln (Exsudate) eingetragen. Neben reinen Auflösungsreaktionen treten weitere Prozesse wie Adsorption, Ionenaustausch, Komplexierung und Bildung von Präzipitaten auf. Diese Prozesse können gleichzeitig oder nacheinander ablaufen.

\subsection{Experimentelle Untersuchungen und natürliche Reaktionsmechanismen}

Silikatverwitterung ist in der Vergangenheit sowohl in Laborexperimenten als auch in Felduntersuchungen betrachtet worden.

Die einzelnen Arbeiten mit Laborexperimenten unterscheiden sich u.a. durch das mineralische Ausgangsmaterial, die Vorbehandlung der Mineralproben, die Zusammensetzung der Verwitterungslösungen, die verwendeten Versuchsreaktoren, das Mineral/Lösungs-Verhältnis und die Versuchstemperatur. Trotz dieser Unterschiede handelt es sich bei den einzelnen Experimenten um gut definierte Systeme. Ihr Nachteil ist aber, daß der experimentelle Ansatz die natürlichen Bedingungen nur sehr eingeschränkt widerspiegelt.

In den Felduntersuchungen gibt es ebenfalls deutliche Unterschiede. Zunächst muß hier ein Wassereinzugsgebiet definiert werden, und es müssen u.a. Annahmen über den Anteil der 
verwitterbaren Minerale, die mit dem Wasser in Kontakt stehende Festkörperoberfläche und den Einfluß durch Flora und Fauna getroffen werden. Der Vorteil dieses Untersuchungsansatzes ist, daß er eine realistische Größe der natürlichen Verwitterungsgeschwindigkeiten geben sollte. Die gewonnenen Geschwindigkeiten sind aber nur so gut, wie die in das System gesteckten Annahmen. Ein weiterer Nachteil ist, daß die ermittelten Verwitterungsgeschwindigkeiten nur für das jeweilige Untersuchungsgebiet gelten und keine Informationen über die an der Verwitterung beteiligten Mechanismen gewonnen werden können.

Wie in zahlreichen Arbeiten beschrieben (u.a. Stumm \& Morgan 1996), führen beide Untersuchungsansätze zu Diskrepanzen der Auflösungsgeschwindigkeiten von Mineralen. Dabei sind die in Felduntersuchungen gefundenen Auflösungsgeschwindigkeiten immer geringer als die in Laborexperimenten beobachteten. Diese Diskrepanzen sind auf eine Vielzahl von Faktoren zurückzuführen, die in Laborexperimenten nur eingeschränkt nachvollziehbar sind. So lassen sich z.B. Wegsamkeiten, Benetzbarkeiten und der Wechsel zwischen Lösungszufuhr und Trockenfallen von Böden nur schlecht simulieren. Weitere kritische Faktoren sind der pH-Wert, $\mathrm{P}_{\mathrm{CO} 2}$, Benetzungsfläche der Mineralkörner, Konzentration von anorganischen Säuren und komplexierenden Liganden, Salinität, Temperatur, Lösungsaustausch zwischen Porenräumen, Reaktivität der Mineraloberflächen, Oberflächenladungen, Oberflächenbeläge (insbesondere von Al- und Fe-Oxiden-Hydroxiden) (Anbeek 1993, Casey et al. 1993, Drever 1997, Drever \& Clow 1995, Furrer et al. 1993, Schnoor 1990, Sverdrup \& Warfvinge 1988, Swoboda-Colberg \& Drever 1993, Velbel 1993, u.a.).

Trotz der Diskrepanzen in den Auflösungsgeschwindigkeiten aus Felduntersuchungen und Laborexperimenten sowie den nur unzureichenden Möglichkeiten, natürliche Systeme im Experiment zu simulieren, sind Laborexperimente für die Untersuchung der einzelnen an der Verwitterung beteiligten Prozesse unverzichtbar. Aus den experimentell gewonnenen Auflösungsgeschwindigkeiten können Rückschlüsse auf die Reaktionsmechanismen gezogen und Verwitterungsmodelle entwickelt werden. Bei der Durchführung von Laborexperimenten ist daher auf möglichst naturnahe Versuchsbedingungen bei gleichzeitig gut definierten Systemen zu achten.

\subsection{Experimenteller Ansatz}

Wegen ihrer relativ großen Oberfläche sind Schichtsilikate maßgeblich am Stoffumsatz in Böden beteiligt. Zudem besitzen einige Schichtsilikate eine hohe Ionenaustauschkapazität. Für die experimentellen Untersuchungen wurden daher die für Böden wichtigsten Schichtsilikate ausgewählt. 
Über die Wechselwirkungen zwischen Schichtsilikaten und wäßrigen Lösungen gibt es zahlreiche Untersuchungen (Carroll \& Walther 1990, Kalinowski \& Schweda 1996, Knauss \& Wolery 1989 u.a.). Aber nur eine begrenzte Anzahl befaßt sich mit den Prozessen in verdünnten Lösungen, wie sie im oberflächennahen Bereich auftreten.

Im Mittelpunkt der Untersuchungen stehen daher Prozesse, die zur Mobilisierung und zur Ausfällung von gelöstem oder partikulärem (kolloidalem) Material führen, wie sie bei der Verwitterung in Bodenhorizonten auftreten. Hierzu soll die Geschwindigkeit der Mineralauflösung unter kontrollierten Randbedingungen in möglichst monomineralischen Systemen unter Verwendung naturnaher Lösungszusammensetzungen ermittelt werden. Einzelne Variablen wie pH-Wert, Säure-Anionen (Sulfat, Nitrat) oder das Vorhandensein organischer Komponenten werden isoliert betrachtet. Um den in Böden auftretenden Porenraumsituationen Rechnung zu tragen, werden die Experimente sowohl in Batch- als auch in Durchfluß-Reaktoren durchgeführt. Sie können als Grenzfälle geschlossener und offener Systeme betrachtet werden.

Die meisten Experimente wurden im stark sauren pH-Bereich (1-3) durchgeführt. Unter diesen Bedingungen erzielt man einen höheren Umsatz in experimentell besser zugänglichen Reaktionszeiten als im schwach sauren pH-Bereich. Simultan mit dem Auflösungsprozeß ablaufende Reaktionen, wie Adsorption oder Präzipitatbildung, können in diesem stark sauren pH-Bereich weitgehend ausgeschlossen werden, so daß die einzelnen Auflösungsprozesse betrachtet werden können. In der Literatur finden sich durchaus Beispiele für die Bedeutung von Auflösungsversuchen im niedrigen pH-Bereich um 2. So haben z.B. Galan et al. (1999) die Auswirkungen von sauren Drainagewässern aus Lagerstätten oder von sauren Flußwässern (Rio Tinto, Südspanien) auf die in diesen Wässern suspendierte Materie beschrieben. Dabei wurde beobachtet, daß die Tonminerale unter diesen Bedingungen stark angegriffen oder vollständig aufgelöst wurden. 


\section{Theoretische Grundlagen}

\subsection{Allgemeines}

Die Auflösung im sauren Milieu ist ein protonenkontrollierter Prozeß, der im wesentlichen in 3 aufeinanderfolgenden Schritten erfolgt (vgl. Lasaga 1981, Wieland et al. 1988 und Zysset \& Schindler 1996):

1. Schnelle Adsorption von Reaktanten,

2. Koordinationsreaktionen der Oberflächenspezies

3. langsame Loslösung der Oberflächenspezies von der Mineraloberfläche

In Abbildung 2-1 sind diese 3 Schritte am Beispiel des Kaolinits verdeutlicht.

Im Fall der „Gibbsit”-Oberfläche werden drei Protonen benötigt, um eine Al(III)-Gruppe aus der Fläche herauszulösen. Im Fall der „Kanten”-Fläche wird hierfür nur ein Proton benötigt. Nach Herauslösen von $\mathrm{Al}(\mathrm{III})$ erfolgt das Freisetzen von $\mathrm{Si}(\mathrm{OH})_{4}$ als schneller Folgeschritt.

Allgemein kann der Vorgang der oberflächenkontrollierten Auflösung nach Stumm (1992) in zwei Umsatzgleichungen wie folgt dargestellt werden:

Oberflächenposition + Reaktanten $\left(\mathrm{H}^{+}, \mathrm{OH}^{-}\right.$, Liganden $) \stackrel{\text { schnell }}{\longrightarrow}$ Oberflächenspezies (2-1)

$$
\text { Oberflächenspezies } \frac{\text { langsam }}{\text { Freisetzung Metall-Kation }} \text { Metall-Kation (aq) (hydratisiert) }
$$

Im ersten Schritt wird die Auflösungsreaktion durch Oberflächenkoordinationen mit den Reaktanten $\left(\mathrm{H}^{+}, \mathrm{OH}^{-}\right.$oder Liganden) eingeleitet. Diese wirkt polarisierend und führt zum Bruch der Metall-Sauerstoff-Bindungen im Kristallgitter. In dieser Abfolge der Reaktionen ist die Auflösungsrate eines Minerals proportional der Konzentration der Oberflächenspezies. Die Gesamtauflösungsrate R setzt sich aus dem Beitrag jedes beteiligten Reaktanten zusammen und ist gegeben durch:

$$
\mathrm{R}=\mathrm{k}_{\mathrm{H}}^{\prime}\left(\mathrm{C}_{\mathrm{H}}^{\mathrm{s}}\right)^{\mathrm{j}}+\mathrm{k}_{\mathrm{OH}}^{\prime}\left(\mathrm{C}^{\mathrm{s}} \mathrm{OH}\right)^{\mathrm{i}}+\mathrm{k}_{\mathrm{L}}^{\prime}\left(\mathrm{C}_{\mathrm{L}}^{\mathrm{s}}\right)+\mathrm{k}_{\mathrm{H}_{2} \mathrm{O}}
$$

Dabei steht k' für die Geschwindigkeitskonstante und $C^{s}$ für die Konzentration des jeweils im Index bezeichneten Reaktanten an der Mineraloberfläche. Das L bezeichnet stellvertretend einen Liganden. Der Term $\mathrm{k}^{\prime} \mathrm{H}_{2} \mathrm{O}$ beschreibt den Hydratationseffekt und spiegelt den $\mathrm{pH}$ unabhängigen Anteil der Auflösungsrate wider (Stumm 1992). Wird die Auflösungsreaktion von Mineralen durch einen Mechanismus gesteuert, so sind die Exponenten i und j gerade Zahlen, die im einfachen Fall der Ladung des Zentralions entsprechen. 
a "Gibbsit"-Oberfläche

b "Kanten"-Oberfläche
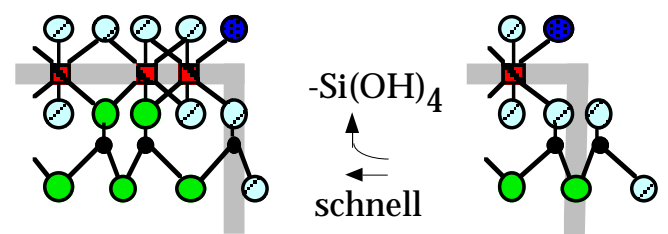

schnell $\downarrow \mathbf{\nabla}+3 \mathrm{H}^{+}$
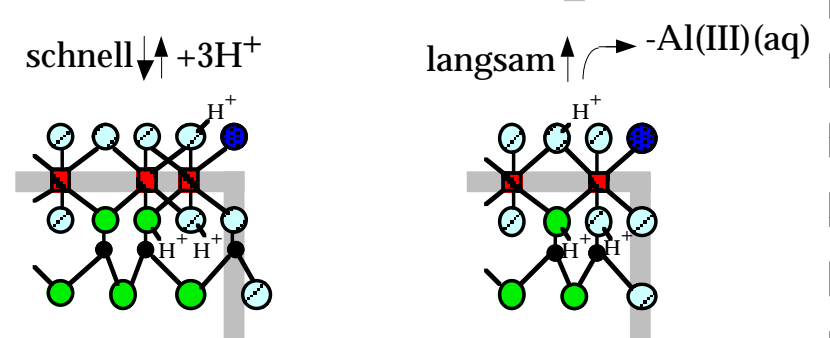

langsam $\downarrow \smile-\mathrm{Al}(\mathrm{III})(\mathrm{aq})$

schnell $\downarrow+3 \mathrm{H}^{+}$
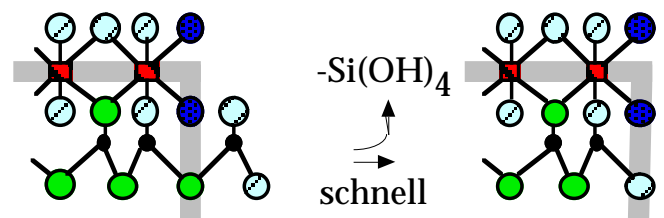

$\Delta \mathrm{Al} \bullet \mathrm{Si} \quad \mathrm{OO}$
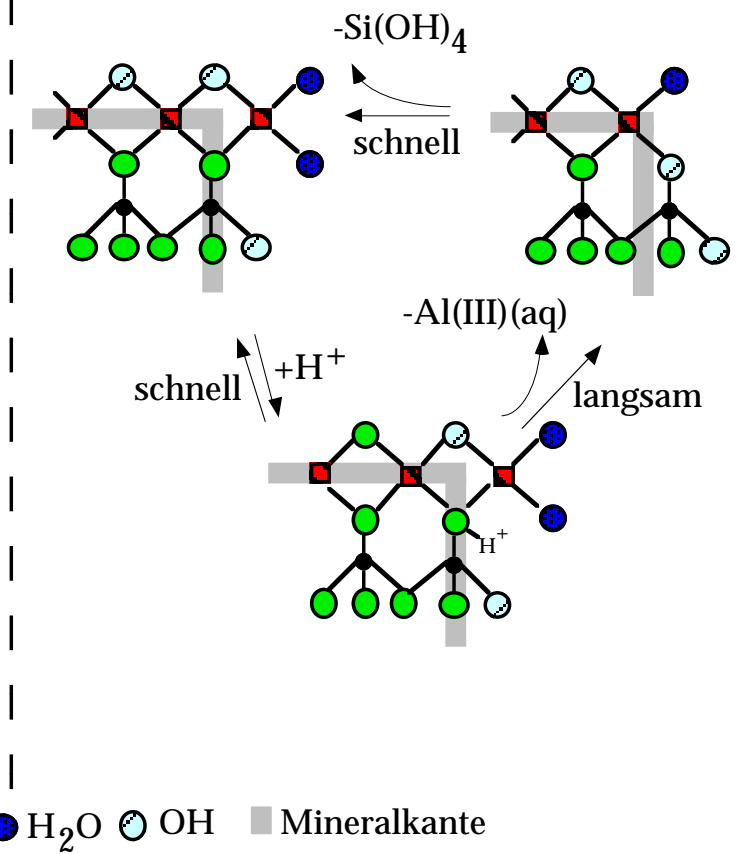

$-\mathrm{Al}(\mathrm{III})(\mathrm{aq})$

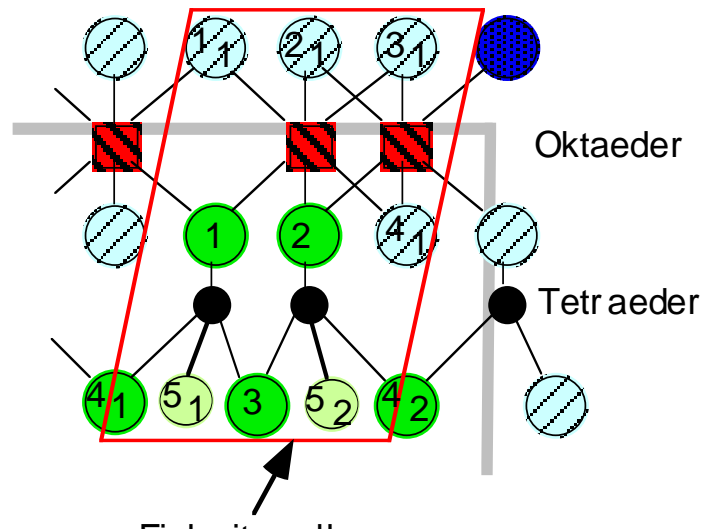

$\mathrm{Al}_{2}(\mathrm{OH})_{4}\left[\mathrm{Si}_{2} \mathrm{O}_{5}\right]$

$\mathrm{OH}: 1_{2}, 2_{2}, 3_{2}, 4_{2}$ liegen außerhalb der Bildebene und sind jeweils deckungsgleich mit den Positionen $1_{1}, 2_{1}, 3_{1}, 4_{1}$

O: $\quad 5_{1}$ und $5_{2}$ liegen ebenfalls außerhalb der Bildebene; sie sind in den obigen Abbildungen nicht dargestellt

Abb. 2-1: Auflösung von Kaolinit nach Stumm (1992). 
Treten mehrere Prozesse simultan auf, so sind die Exponenten gebrochene Zahlen. Die Exponenten werden auch als Reaktionsordnung bezeichnet.

Im einfachsten Fall kann z.B. eine rein protonengeförderte Lösungsrate angenommen werden, wobei die Gleichung 2-3 sich wie folgt vereinfachen läßt :

$$
\mathrm{R}_{\mathrm{H}}=\mathrm{k}_{\mathrm{H}}^{\prime}\left(\mathrm{C}_{\mathrm{H}}^{\mathrm{S}}\right) \quad(2-4) \quad \text { und } \quad \mathrm{C}_{\mathrm{H}}^{\mathrm{S}}=\frac{\left.\left[\Sigma \mathrm{H}^{+}\right]-\left[\mathrm{H}^{+} \text {(Lösung }\right)\right]}{\text { Mineraloberfläche }}
$$

$\mathrm{C}^{\mathrm{s}}{ }_{\mathrm{H}}$ steht in diesem speziellen Fall für die Konzentration der $\left(\equiv \mathrm{MeOH}_{2}{ }^{+}\right)$-Gruppen der Mineraloberfläche.

Wie aus Gleichung 2-5 ersichtlich, geht in die Bestimmung der Auflösungsrate die Oberfläche der Minerale ein. Problematisch ist hierbei, daß für die Betrachtung der Mineralauflösung zwischen gesamter Mineraloberfläche und reaktiver Oberfläche unterschieden werden muß. Für Schichtsilikate wird allgemein angenommen, daß die Auflösung hauptsächlich über die Kanten erfolgt (u.a. Wieland \& Stumm 1992). Eine Ermittlung dieser Kantenflächen ist aber nicht exakt möglich. Bei Kaoliniten kann der Anteil der Seitenflächen an der Gesamtoberfläche zwischen 20 und 47\% variieren (Nagy 1995).

Die protonengeförderte Lösungsrate $\mathrm{R}_{\mathrm{H}}$ kann auch pauschal auf die freien Wasserstoffionen bezogen werden:

$$
\mathrm{R}_{\mathrm{H}}=\mathrm{k}_{\mathrm{H}}^{\prime}\left(\mathrm{H}^{+}\right)^{\mathrm{n}}
$$

Der Wert $\mathrm{n}$ gibt die Reaktionsordnung in bezug auf die Wasserstoffionenkonzentration an. Durch Logarithmieren läßt sich eine lineare Beziehung zwischen Lösungsrate $\mathrm{R}_{\mathrm{H}}$ und dem $\mathrm{pH}$ Wert der Lösung herstellen:

$$
\lg \mathrm{R}_{\mathrm{H}}=-\mathrm{n} \mathrm{pH}+\lg \mathrm{k}_{\mathrm{H}}^{\prime}
$$

wobei $\mathrm{n}$ in Form der Steigung aus einer entsprechenden Auftragung ermittelt werden kann.

\subsection{Bestimmung von Auflösungsraten aus Lösungs-Experimenten}

Die Ermittlung von Auflösungsgeschwindigkeiten basiert auf der Freisetzungsrate der Gitterbausteine in die Lösung. Bei den einzelnen Silikaten sind dies vor allem $\mathrm{Si}, \mathrm{Al}$ und $\mathrm{Mg}$. Bei kontantem pH-Wert erhält man für die Änderung der Konzentrationen von Si bzw. Al als Funktion der Zeit:

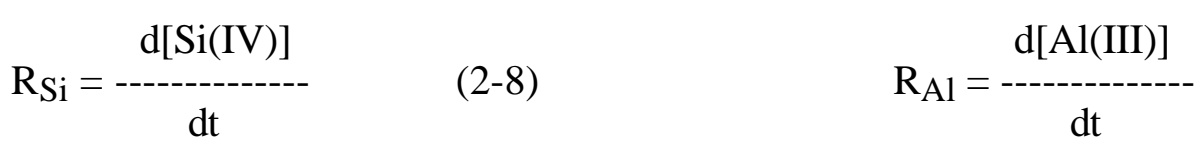

Weil Mineralauflösung, wie beschrieben, ein oberflächenkontrollierter Prozeß ist, geben die meisten Autoren Auflösungsraten in $\left[\mathrm{mol} /\left(\mathrm{m}^{2} \bullet \mathrm{h}\right)\right]$ an. Da die Ermittlung der Oberfläche aber, 
wie bereits erwähnt, problematisch ist, werden die Auflösungsraten für die durchgeführten Experimente in Anlehnung an Furrer et al. (1993) bzw. Zysset \& Schindler (1996) in $[\mathrm{mol} /(\mathrm{g} \bullet \mathrm{h})]$ angegeben. Die Ermittlung der Auflösungsraten aus den Batch- bzw. DurchflußExperimenten ist in Abbildung 2-2 für typische Experimente dargestellt.

In Durchfluß-Experimenten erreichen die durch Mineralauflösung in Lösung gegangenen Ionen mit der Zeit einen stationären Zustand (,steady state”). Die in diesem Bereich vorhandene Konzentration ist in Abbildung 2-2a mit „Cst” bezeichnet. Zur Bestimmung der Auflösungsrate wird der Mittelwert der Konzentrationen in diesem Bereich dividiert durch die Verweilzeit der Lösung im Reaktor und die Partikelkonzentration.

In Batch-Experimenten steigt die Konzentration mit der Zeit stetig an (Abb. 2-2b). Zur Bestimmung der Auflösungsrate wird die daraus resultierende Steigung durch die Partikelkonzentration dividiert.

(a)

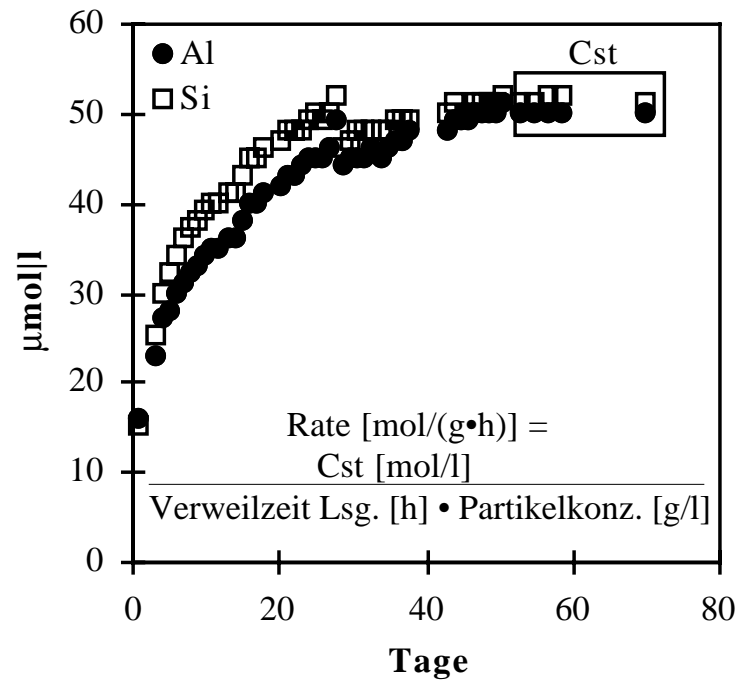

(b)

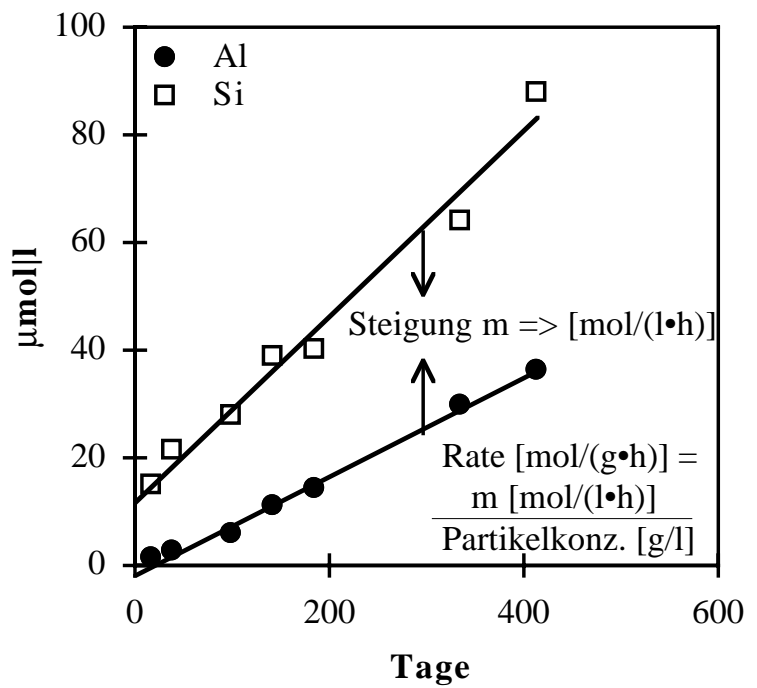

Abb. 2-2: Bestimmung von Auflösungsraten am Beispiel eines Durchflu $\beta-(a)$ und eines BatchExperimentes (vgl. Versuche IV250(1) (a) bzw. 8 (b) im Anhang) 


\subsection{Ermittlung weiterer Kenngrößen aus den Experimenten}

Weitere Informationen über das Auflösungsverhalten der Minerale kann man aus dem Verhältnis der in Lösung gegangenen Ionen zueinander und der Reaktionsordnung gewinnen. Bei stöchiometrischer Auflösung entspricht das Verhältnis der Gitterbausteine in der Lösung dem im Festkörper.

Nichtstöchiometrische Auflösung in Experimenten kann verschiedene Gründe haben, wie z.B. (Zusammenstellung aus Bloom \& Nater 1991):

1. Hydrolyse und Austausch ladungsausgleichender Kationen in der äußersten Mineral-Lage

2. Bildung sekundärer Präzipitate

3. Bildung von verarmten Schicht-Lagen durch bevorzugtes Herauslösen einzelner Ionenspezies

4. Bildung von stark hydratisierten Resten des Silikat-Netzwerks

5. Vorhandensein weiterer Mineral-Komponenten anderer Zusammensetzung

Wie bereits in Gleichung 2-7 gezeigt, kann die Abhängigkeit der Auflösungsrate vom pH-Wert in Form der Reaktionsordnung $\mathrm{n}$ abgeleitet werden. Diese wird zwar durch gelöste Komponenten beeinflusst, stellt aber dennoch eine gute Möglichkeit dar, Daten aus der Literatur untereinander zu vergleichen.

Besondere Beachtung finden hierbei die auf Basis der $\mathrm{R}_{\mathrm{Si}}$-Daten ermittelten Reaktionsordnungen. Zum einen ist Si ein Hauptgitterbaustein der Silikate, und zum anderen liegt es in den verwendeten Lösungen als ungeladener $\mathrm{Si}(\mathrm{OH})_{4}$-Komplex vor. Im Gegensatz dazu liegt das $\mathrm{Al}$ in den verwendeten Lösungen 3-fach positiv geladenen als $\mathrm{Al}^{3+}$ bzw. $\mathrm{Al}\left(\mathrm{H}_{2} \mathrm{O}\right)_{6}{ }^{3+}$ vor. Aufgrund der geringeren elektrostatischen Kräfte sollte der $\mathrm{Si}(\mathrm{OH})_{4}-\mathrm{Komplex}$ geringer mit den Partikeloberflächen oder den in den Lösungen vorhandenen Ionen reagieren als das Al-Ion bzw. der Al-Komplex.

\subsection{Fehlerbetrachtung der berechneten Kenngrößen}

Sowohl für die Batch- als auch für die Durchfluß-Experimente wurde für die angegebenen Auflösungsraten ein Fehler von 15\% ermittelt. Die Fehlerbereiche sind in den ensprechenden Abbildungen graphisch dargestellt.

Für die Batch-Experimente ergibt sich der Fehler aus der Variation der Steigung - Konzentration mit der Zeit -, welche wiederum hauptsächlich vom Fehler der Konzentrationsbestimmung abhängt. Im Fall der Durchfluß-Experimente setzt sich der Fehler zusammen aus der Schwankung der Elementkonzentration im „steady state” inkl. des Fehlers der 
Konzentrationsbestimmung sowie des Fehlers der Verweilzeit, welcher abhängt von der Schwankung der Durchflußgeschwindigkeit.

Der Fehler der Partilkelkonzentration ist definiert durch den Einwaagefehler und kann vernachlässigt werden.

Der Fehler für die Reaktionsordnung n resultiert direkt aus der Variation der Steigung - $\lg ($ Rate $)$ gegen pH-Wert -. Er wird in den entsprechenden Tabellen zusammen mit der Reaktionsordnung angegeben. 


\section{Analyseverfahren}

\subsection{Festkörper}

Die chemische Charakterisierung der Festkörper erfolgte mit einem Röntgenfluoreszenzspektrometer (RFA, Philips PW 1480/X automated sequential spectrometer) an LithiumboratSchmelztabletten (Hartmann 1994).

Die mineralogische Zusammensetzung wurde an Texturpräparaten nach der „Filter-Membrane Peel Technique" (Drever 1973) mit einem Röntgendiffraktometer (Philips X’Pert-MPD/MRD) ermittelt. Zusätzlich erfolgte eine Untersuchung mittels FouriertransformationsInfrarotspektroskopie (FT-IR, Perkin Elmer 1600 Series FTIR) an KaliumbromidPreßtabletten. Eine ausführliche Beschreibung dieser Methode findet sich bei Flehmig \& Kurze (1973) und Wilson (1987).

Die Oberflächenbeschaffenheit wurde mit einem Feldemissions-Rasterelektronenmikroskop (FEM, LEO GEMINI 1530) dokumentiert. In einem Einzelfall erfolgte zusätzlich eine Untersuchung mit einem Raster-Kraftmikroskop (AFM, Digital Instruments Nanoscope II) im Institut für Nichtmetallische Werkstoffe in Clausthal-Zellerfeld.

\subsection{Lösungen}

Zum Einstellen der pH-Werte in den Input-Lösungen und für die Messung der OutputLösungen wurde ein pH-Meter der Firma Sentron mit ISFET- (Ion Sensitive Field Effect Transistor) Sensor eingesetzt. Dieser besteht aus einem Transistor und einer ionenselektiven Membran, die elektrolytfrei und dadurch kontaminationsfrei arbeitet. Die Kalibrierung wurde mit entsprechenden Pufferlösungen (Merck Darmstadt) durchgeführt.

Für die chemische Analyse der Lösungen wurde ein Atomemissionsspektrometer mit induktiv gekoppeltem Plasma (ICP-OES, Perkin Elmer Optima 3300 DV mit Probenautomat AS91) verwendet bzw. ein Graphitrohrofen-Atomabsorptionsspektrometer (GF-AAS, Unicam 939 Solar System mit GF90 Graphitrohrofeneinheit und Probenautomat FS90). Eine Beschreibung der Meßverfahren ist bei Heinrichs \& Herrmann (1990) bzw. Boss \& Fredeen (1997) gegeben. Die Kalibrierung erfolgte mit Maßlösungen und Multielementstandardlösungen der Bernd Kraft GmbH (Duisburg-Neumühl) und der Firma Merck (Darmstadt). Die GF-AAS wurde nur für die Analysen von Al und Si in den Lösungen von Experiment II (Kaolinit) verwendet. Da diese Lösungen $\mathrm{H}_{2} \mathrm{SO}_{4}$-sauer waren, sind entsprechende Kalibrationslösungen verwendet worden. Für die Analysen mit der ICP-OES wurden in allen Fällen $\mathrm{HNO}_{3}$-saure Kalibrationslösungen eingesetzt. Probleme durch abweichende Säure-Konzentrationen oder Säure-Arten in den 
Probe-Lösungen konnten nicht festgestellt werden. Die in den Lösungen gemessenen Elemente mit ihren Meßbereichen sind in Tabelle 3-1 aufgeführt.

Tab. 3-1: Übersicht der in den Lösungen gemessenen Elemente und die zugehörigen Meßbereiche mit der ICP-OES und GF-AAS.

\begin{tabular}{|c|c|c|}
\hline \multicolumn{3}{|c|}{ ICP-OES } \\
\hline Element & Min $\mu \mathbf{g} / \mathbf{l}(\mu \mathbf{m o l} / \mathbf{l})$ & Max mg/l (mmol/l) \\
\hline \hline $\mathrm{Al}$ & $10(0,37)$ & $500(18,53)$ \\
\hline $\mathrm{Fe}$ & $10(0,18)$ & $500(8,95)$ \\
\hline $\mathrm{K}$ & $100(2,6)$ & $100(2,6)$ \\
\hline $\mathrm{Mg}$ & $20(0,82)$ & $500(20,57)$ \\
\hline $\mathrm{Na}$ & $100(4,4)$ & $100(3,56)$ \\
\hline $\mathrm{Si}$ & $10(0,36)$ & $\mathbf{M a x} \mu \mathbf{g} / \mathbf{l}(\mu \mathbf{m o l} / \mathbf{l})$ \\
\hline $\mathrm{Element}$ & $\mathbf{G F}-\mathbf{A A S}$ & $1000(37,06)$ \\
\hline $\mathrm{Al}$ & $10(0,37)$ & $1000(35,61)$ \\
\hline \hline $\mathrm{Si}$ & $10(0,36)$ & \\
\hline
\end{tabular}

\subsection{Fehlerbetrachtung}

Für die verwendete $\mathrm{pH}$-Elektrode gibt der Hersteller einen Fehler von $\pm 0,01 \mathrm{pH}$ an. In den Batch-Experimenten kann aber eine Drift der $\mathrm{pH}$-Werte durch den Verbrauch von $\mathrm{H}^{+}$-Ionen während der Reaktion auftreten. Dieser Effekt kann vor allem bei $\mathrm{pH}-$ Werten > 3 beträchtlich sein. In der Ergebnisdarstellung wird in Fällen besonders deutlicher pH-Drift diese in den Grafiken vermerkt. Ein Verbrauch von $\mathrm{H}^{+}$-Ionen wirkt sich in den Durchfluß-Experimenten nicht aus, da fortlaufend frische Lösung nachgeliefert wird.

Die Richtigkeit der Analysen mit der RFA, ICP-OES und GF-AAS wurde durch Messungen von institutsinternen (TW, BB) und internationalen (SLRS-3, JB-3, JR-2) Referenzmaterialien kontrolliert. Im Falle der Lösungsanalytik mittels ICP-OES und GF-AAS wurde sowohl auf den Wasserstandard SLRS-3 als auch auf Aufschlußlösungen der Gesteinsstandards JB-3 und 
JR-2 zurückgegriffen. Letztere dienten vor allem zum Vergleich sehr hoch konzentrierter Output-Lösungen, wie sie in einigen Batch-Experimenten auftraten.

Neben den Fehlern der Analyseverfahren müssen auch die experimentell bedingten Fehler berücksichtigt werden. Im Fall der Input-Lösungen kann dieser durch Verwendung möglichst reiner Chemikalien und inerter Materialien für deren Aufbewahrung vernachlässigt werden. Im Falle der Batch-Experimente konnte weder ein Einfluß durch den Reaktor noch durch die verwendeten Filter beobachtet werden. Bei den Durchfluß-Experimenten hingegen setzte der Reaktor in geringen Mengen Ca und Zn frei. Ihre Auswirkung auf die Experimente scheint aber vernachlässigbar. Ein Einfluß der Filtermembranen konnte nicht festgestellt werden.

Unter Einbeziehung der genannten Fehlerquellen ergibt sich für die angegebenen Konzentrationen ein relativer Gesamtfehler von maximal 10\%, der aber im Bereich der Bestimmungsgrenzen der einzelnen Analyseverfahren bis auf 50\% ansteigen kann. 


\section{Experimenteller Aufbau}

\subsection{Zusammenstellung verschiedener experimenteller Methoden}

Einen Überblick über verschiedene experimentelle Methoden zur Gewinnung kinetischer Parameter geben u.a. Amacher (1991) und Stumm \& Morgan (1996). Ebenso findet sich bei Amacher (1991) eine Zusammenstellung der Vor- und Nachteile von Batch- bzw. DurchflußReaktoren. Im folgenden werden die verschiedenen Methoden anhand ihrer wichtigsten Merkmale kurz vorgestellt.

\section{Batch Methode}

Die einfachste Methode zur Untersuchung kinetischer Parameter stellt die Arbeit mit BatchReaktoren dar. In bezug auf $\mathrm{H}_{2} \mathrm{O}$ stellt diese Methode ein geschlossenes System dar. Ein BatchReaktor besteht aus einem einfachen Glas- oder Kunststoffgefäß, wobei die Materialart sich nach den zu untersuchenden Lösungen richtet. In diesem Gefäß wird durch Rühren des Festkörper-Lösungsgemisches oder Schütteln bzw. Rotieren des Gefäßes eine ständige Durchmischung des zu untersuchenden Materials erreicht. In der Regel können auf diese Weise Diffusionsprozesse unterdrückt werden. Die physiko-chemischen Parameter, wie pH, Ionenstärke, $\mathrm{P}_{\mathrm{CO} 2}$ sind nachregelbar, wobei aber in das bestehende System eingegriffen werden muß (z.B. durch Zugabe von Säure).

Im Gegensatz zu den Durchfluß-Methoden werden die gelösten Komponenten nicht durch nachströmende Lösung aus dem System ausgetragen und können akkumulieren. Bei genügend hohen Konzentrationen der gelösten Komponenten müssen daher mögliche Rückreaktionen oder die Bildung von sekundären Phasen entsprechend berücksichtigt werden.

\section{Durchfluß Methoden}

Die Durchfluß-Methoden stellen in bezug auf $\mathrm{H}_{2} \mathrm{O}$ ein offenes System dar. Die verschiedenen Methoden unterscheiden sich dabei im wesentlichen durch die Art der Suspendierung des Probenmaterials. Außerdem sind die Parameter wie $\mathrm{pH}$, Ionenstärke usw. variabel einstellbar. Durch die Wahl der Fließgeschwindigkeit der Lösungen durch den Reaktor können die Konzentrationen in den Output-Lösungen beeinflußt werden. Die während der Reaktion gelösten Komponenten werden ständig aus dem System ausgetragen, womit die Berücksichtigung von Rückreaktionen oder die Bildung sekundärer Phasen in der Regel nicht notwendig ist. 


\section{„Thin-disk" Methode}

Diese Methode wird in der Literatur auch als „miscible-displacement” oder „continuous-flow” Methode bezeichnet. Die Probe wird als dünne Schicht auf eine poröse Membran gegeben, welche in einem Halter fixiert ist. Mittels einer Pumpe wird mit konstanter Fließgeschwindigkeit Lösung durch die Membran gepumpt und nach dem Durchströmen der Probe in Aliquoten gesammelt. Ein Vorteil dieser Methode ist ihr einfacher Aufbau. Aufgrund der kurzen Verweildauer der Lösung in der Probe können schnelle Reaktionen untersucht werden. Der Nachteil besteht darin, daß die Probe nicht gerührt wird und ein ungleichmäßiger Kontakt zwischen Festkörper und Lösung besteht. Außerdem kann die Lösung auf bevorzugten Wegsamkeiten die Probe durchfließen und so nur mit einem Teil des Festkörpers reagieren.

\section{,Stirred-flow" Methode}

Der Aufbau dieser Methode ist dem der „thin-disk” Methode sehr ähnlich. Statt der Membran, auf der die Probe verteilt wird, befindet sich zwischen Pumpe und Fraktionssammler ein Gefäß. In diesem wird das zu untersuchende Material mittels eines Magnetrührers oder eines Flügelpropellers in einer Lösung suspendiert. Die Verwendung eines solchen Reaktors ermöglicht die Untersuchung verschiedenster Materialien mit einer großen Korngrößenvariabilität. Ein Nachteil ist das mechanische Rühren, wodurch die Probenpartikel zerkleinert werden können.

\section{„Fluidized-bed” Methode (Wirbelschicht Methode)}

Bei der „fluidized-bed” Methode wird die Durchmischung von Partikeln und Lösung im Reaktor nicht durch einen Rührer erreicht, sondern durch einen aufwärtsgerichteten Strom der Input-Lösung. Dieser wird so gewählt, daß er der Sinkgeschwindigkeit der Partikel in der Lösung gerade entspricht. Hierzu wird mit zwei verschiedenen Pumpen gearbeitet. Eine regelt den Lösungstrom zur Suspendierung der Partikel und eine zweite regelt den der OutputLösung, die den Reaktor nach einer definierten Kontaktzeit mit dem Festkörper verläßt. Die Vorteile der „fluidized-bed” Methode sind ähnlich, wie sie bereits bei der „stirred-flow” Methode beschrieben wurden. Darüber hinaus werden die Partikel nicht mechanisch beansprucht. 


\section{„Mixed-flow" Methode}

Bei der „mixed-flow” Methode wird vergleicbar der Batch-Methode ein geschlossener Behälter verwendet. Die Lösung wird nicht kontinuierlich durch das System gepumpt sondern nach einer definierten Kontaktzeit mit dem Festkörper komplett oder teilweise gegen frische Lösung ausgetauscht.

\subsection{Beschreibung der verwendeten experimentellen Methoden}

In der vorliegenden Arbeit wurden in den Experimenten Batch- und Durchfluß-Reaktoren verwendet. Letztere entsprechen der „stirred-flow” Methode, die in der Literatur auch als „flowthrough"- bzw. Durchfluß-Methode bezeichnet wird. Der Aufbau der in dieser Arbeit verwendeten Reaktoren und ihre wesentlichen Merkmale werden in den nächsten beiden Abschnitten beschrieben.

\subsubsection{Geschlossene Systeme - Batch-Experimente}

Geschlossene Systeme können in Batch-Reaktoren simuliert werden. Im Verlauf der Reaktion werden keine Reaktanten durch Lösungszufuhr hinzu- oder abgeführt. Gelöste Komponenten können akkumulieren. Bei entsprechend hoher Konzentration gelöster Ionen können so Bedingungen simuliert werden, wie sie in Feinporen oder im ungesättigten Wasserfluß in Böden vorliegen. Die akkumulierten Produkte können am Festkörper adsorbieren und dadurch die Auflösungsgeschwindigkeit beeinflussen. Außerdem ist bei entsprechender Konzentration die Bildung von Präzipitaten möglich.

Zur Durchführung der Batch-Experimente dienten 100 ml-PE-Weithalsflaschen. Die Lösungen wurden mit Hilfe einer vertikal rotierenden Apparatur (30 Umdrehungen pro Minute) ständig durchmischt und regelmäßig beprobt. Die Beprobung erfolgte mit 5 ml-Kunststoff-Spritzen und einem Spritzenfiltervorsatz mit einer Cellulose-Acetat-Membran bei einer Porengröße von 0,2 $\mu \mathrm{m}$. Die entnommenen Proben wurden bis zur Analyse in Reagenzrohren aus Kunststoff aufbewahrt. Die Messung der pH-Werte erfolgte unmittelbar bei der Probenahme. Der gewonnene Filterrückstand wurde mit destilliertem Wasser von den Filtern in PE-Gefäße überführt. Anschließend erfolgte eine Gefriertrocknung, wodurch weitere Reaktionen mit dem Festkörper verhindert werden sollten. Der Festkörper konnte dann mit optischen Methoden auf Oberflächenveränderungen untersucht werden. Das Filtrat wurde keiner weiteren Behandlung unterzogen.

Um Algenwachstum in den Experimenten weitestgehend zu unterdrücken, wurden sie in dunklen, fensterlosen Räumen durchgeführt. Die Temperatur betrug $20 \pm 2^{\circ} \mathrm{C}$. 


\subsubsection{Offene Systeme - Durchfluß-Experimente}

Experimente in Durchfluß-Reaktoren stellen offene Systeme dar. Im Gegensatz zum geschlossenen System werden gelöste Komponenten durch ständige Lösungszufuhr abtransportiert. In bezug auf Böden entspricht dies etwa Bedingungen im gesättigten Wasserfluß in Grobporen.

Als Reaktoren dienten Ultrafiltrationsgefäße der Firma Amicon® mit einem Volumen von 90 bzw. $250 \mathrm{ml}$. Eine Beschreibung des Reaktors und seiner Teile ist den Abbildungen A-1 und A2 zu entnehmen. Der Durchfluß der Lösungen durch die Reaktoren wurde über eine MehrkanalPeristaltik-Pumpe (Ismatec $\left.{ }^{\circledR}\right)$ gesteuert. Als Schlauchmaterial in der Pumpe diente Tygon®, für die Output-Schläuche PTFE (Polytetrafluorethylen, Teflon ${ }^{\circledR}$ ). Als Behälter für die InputLösungen wurden 500 ml PFA- (Perfluoralkoxy) Flaschen benutzt. Durch die begrenzte Größe der Input-Gefäße wurde regelmäßig frische Lösung angesetzt und ein altern der Lösungen vermieden. Das Auffangen der Output-Lösungen erfolgte in Reagenzrohren aus Kunststoff. Aus der gesammelten Menge pro Zeitintervall wurde für jede Probe die entsprechende Durchflußgeschwindigkeit ermittelt. Wie bei den Batch-Experimenten wurde das gewonnene Filtrat keiner weiteren Behandlung unterzogen.

Am Ausgang des Reaktors wurde die Lösung über eine PTFE-Filter-Membran mit einer Porenweite von 0,2 $\mu \mathrm{m}$ geleitet. Da diese Filter hydrophob sind, mußten sie vor dem Einbau in den Reaktor zunächst mit Alkohol hydrophilisiert werden. Damit kein Alkohol in den Reaktor hineingelangte, wurden die Filter vor dem Einbau mit hochreinem Wasser $\left(\mathrm{H}_{2} \mathrm{O}^{* * * *}\right)$ gespült.

Das Einfüllen der Input-Lösungen in die Reaktoren geschah möglichst luftblasenfrei. Dies war u.a. notwendig, da die Lösungen von unten in die Reaktoren hineingepumpt wurden und diesen über den oben eingebauten Filter wieder verließen. Diese Anordnung gewährleistete auch bei Verwendung sehr feiner Mineralfraktionen im Verlauf lange dauernder Versuche einen uneingeschränkten Filterdurchsatz, ohne mögliche Probleme durch einen Verschluß der Poren mit dem Probenmaterial. Damit in verschiedenen Reaktoren die gleiche Rührgeschwindigkeit vorlag, wurden Rührvorrichtungen angefertigt, mit denen bis zu 6 Reaktoren gleichzeitig betrieben werden konnten.

Das Rühren des Festkörper-Lösungsgemisches stellt eine mechanische Beanspruchung der Partikel dar, die zu deutlicher Oberflächenvergrößerung führen kann. Ein Experiment von Metz \& Ganor (1998) zeigt hierzu einen sichtbaren Einfluß des Suspensionsgrades auf die Auflösungsgeschwindigkeit von Kaolinit. Bei der Einstellung der Rührgeschwindigkeit wurde daher darauf geachtet, daß sämtliches Mineralpulver in Suspension war. Eine mechanische Zerkleinerung des Pulvers durch den Rührvorgang sollte dabei so gering wie möglich gehalten werden. Eine ausreichende Geschwindigkeit, die beide Bedingungen berücksichtigt, konnte mit 
der Einstellung auf 250 Umdrehungen pro Minute erreicht werden. Ein schematischer Aufbau der Apparatur ist in Abbildung 4-1 gezeigt. Außerdem findet sich in Abbildung 4-2 ein Photo des Aufbaus mit der Rührvorrichtung.

Wie die Batch-Experimente wurden auch die Durchfluß-Experimente in dunklen, fensterlosen Räumen durchgeführt. Die Temperatur lag mit $25 \pm 2{ }^{\circ} \mathrm{C}$ etwas über der für die BatchExperimente.

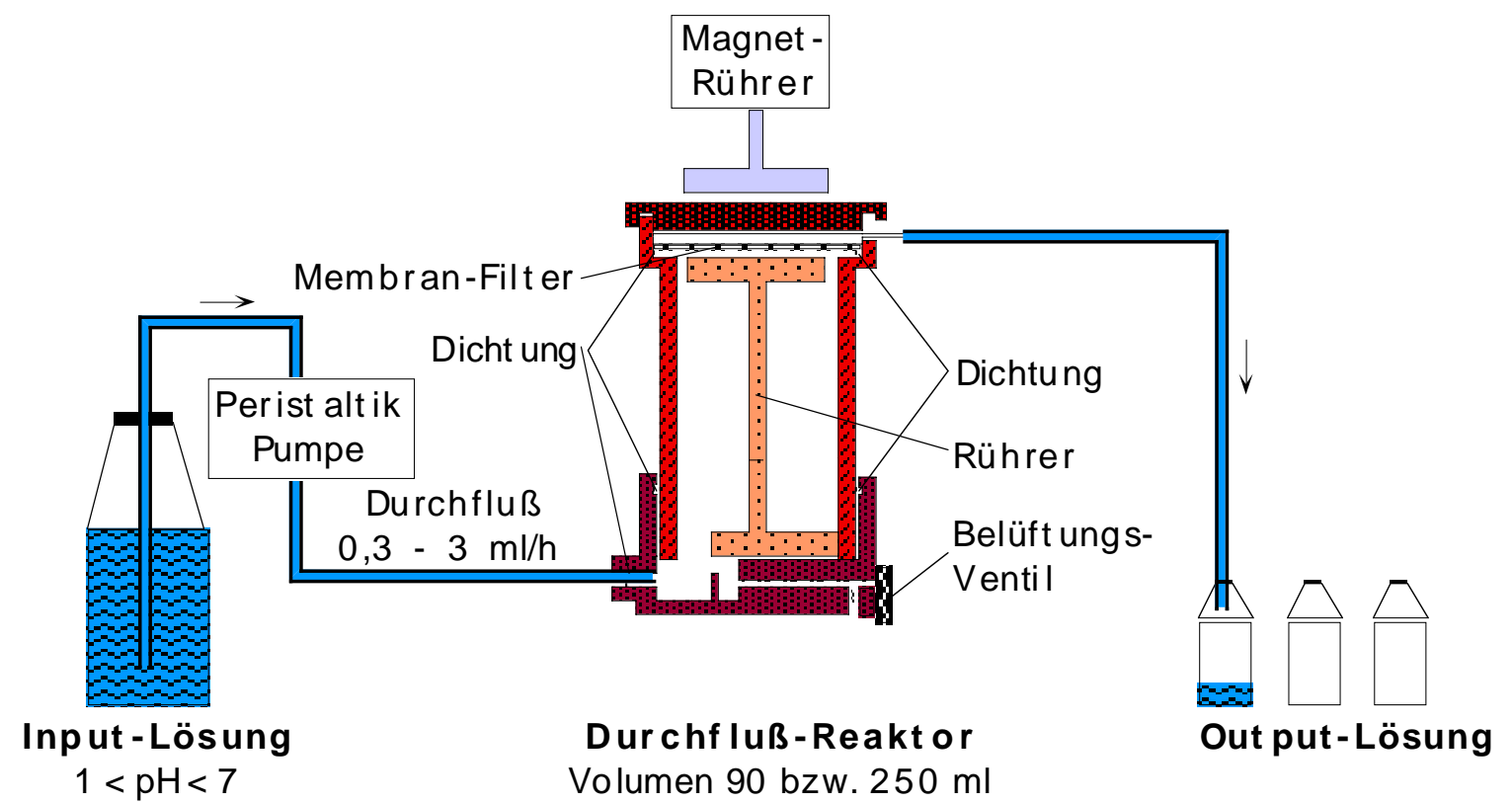

Abb. 4-1: Schematische Darstellung der Durchfluß-Apparatur. 


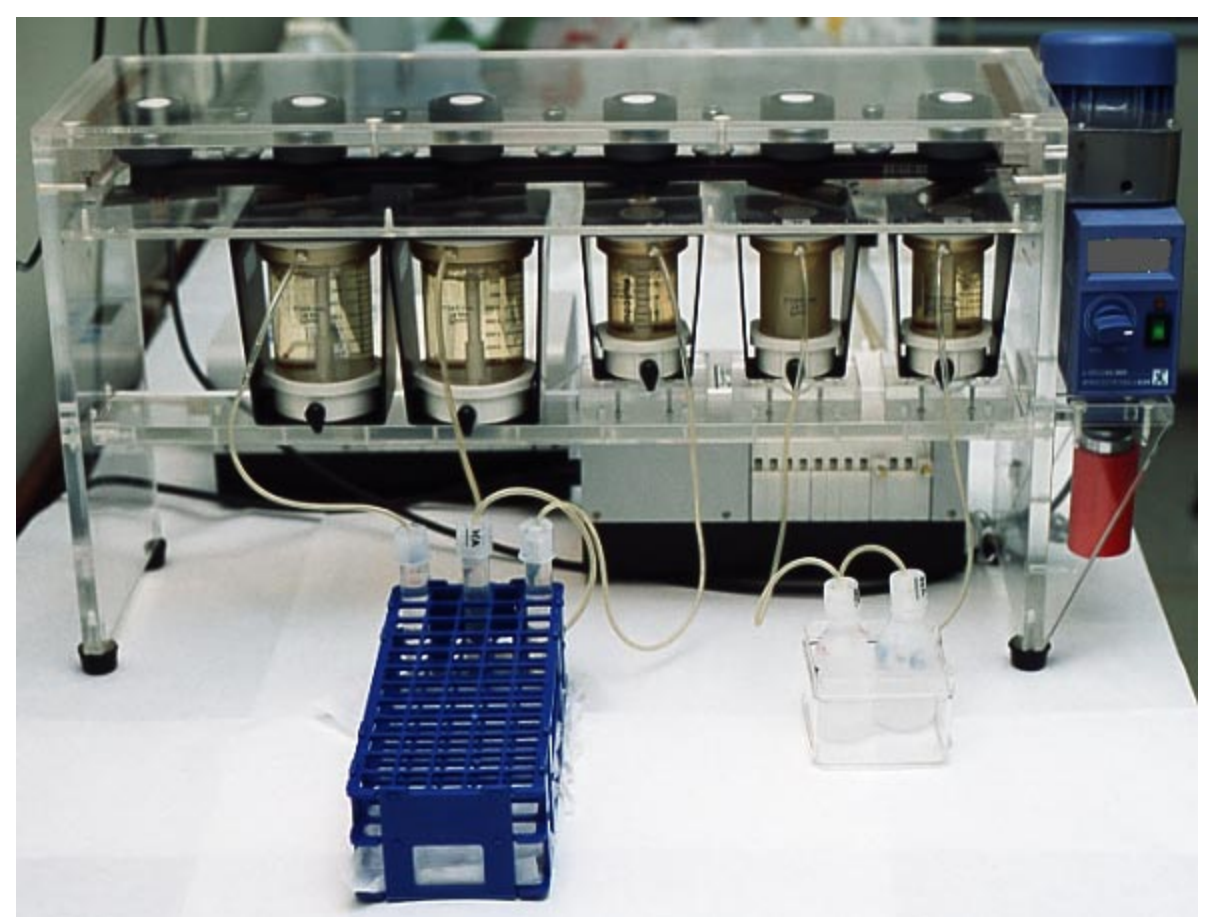

Abb. 4-2: Abbildung der Durchfluß-Reaktoren in der Rührvorrichtung. 


\section{Probenmaterial}

\subsection{Mineralpulver}

In den Experimenten wurden Kaolinit, Illit, Montmorillonit und Serizit als Hauptvertreter der in Böden auftretenden Schichtsilikate eingesetzt. Die in Böden häufig vorkommenden Wechsellagerungsminerale sind durch die Endglieder Illit und Montmorillonit vertreten. Talk wurde als Modellsubstanz für Mg-reiche Schichtsilikate eingesetzt.

Da die verwendeten Mineralpulver aus Lagerstätten stammen, haben sie alle eine breite Streuung der Partikelgröße und einen gewissen Anteil Nebengemengteile. Es wurde daher eine Auftrennung in definierte Korngrößenbereiche mittels Atterbergverfahren und Zentrifugation durchgeführt. Um bei der Korntrennung mögliche chemische Kontaminationen zu vermeiden wurde $\mathrm{H}_{2} \mathrm{O}^{* * * *}$ verwendet. Eine Behandlung mit Ultraschall erfolgte nur für das erstmalige Suspendieren. Jeder weitere Schritt wurde ohne weitere Behandlung mit Ultraschall durchgeführt. Da der verwendete Talk nur in derben Massen vorlag, wurde er zusätzlich gemahlen und als Fraktion 2 - $63 \mu \mathrm{m}$ verwendet. Während der Experimente zeigten sich durch diese Vorbehandlung jedoch Probleme, die im Abschnitt Ergebnisse näher beschrieben werden.

In Tabelle 5-1 ist eine kurze Charakterisierung der verwendeten Mineralproben gegeben. Die für die Minerale angegebenen Elementverhältnisse und Strukturformeln wurden aus der chemischen Analyse berechnet. Für die Berechnung der Strukturformeln wurde die typische Zusammensetzung der Minerale berücksichtigt. Es kann daher zu Unterschieden der molaren Elementverhältnisse auf Basis der Strukturformel bzw. der chemischen Analyse des Festkörpers kommen. In Tabelle 5-1 werden daher sowohl die aus der Strukturformel als auch die aus der chemischen Analyse bestimmten Elementverhältnisse angegeben.

Tab. 5-1: Charakterisierung der verwendeten Mineralproben (HWB: Halbwertsbreite eines RDA-Peaks)

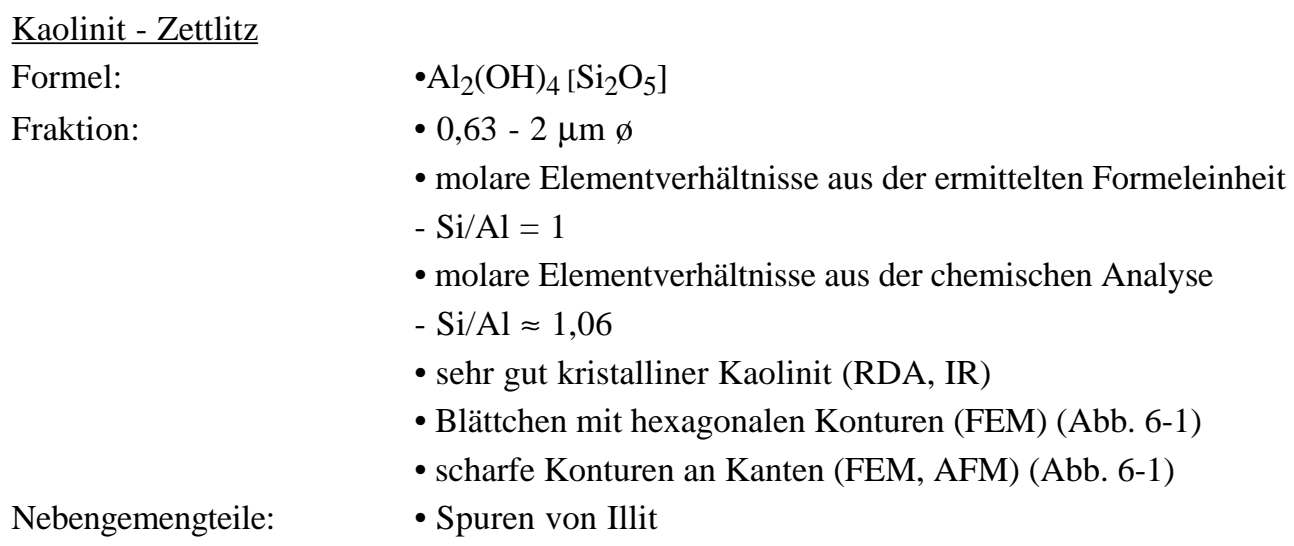


Tab. 5-1: Fortsetzung

Illit H-35 - Fithian/Illinois

\begin{tabular}{|c|c|c|c|c|}
\hline Formel: * & \multicolumn{4}{|c|}{ - $\mathrm{K}_{0,65} \mathrm{Al}_{1,45} \mathrm{Fe}^{3+}{ }_{0,2} \mathrm{Mg}_{0,35}(\mathrm{OH})_{2}\left[\mathrm{Si}_{3,7} \mathrm{Al}_{0,3} \mathrm{O}_{10}\right]$} \\
\hline \multirow[t]{6}{*}{ Fraktion: } & \multicolumn{4}{|l|}{ • $0,63-2 \mu \mathrm{m} \varnothing$} \\
\hline & \multirow{2}{*}{\multicolumn{4}{|c|}{$\begin{array}{l}\text { - sehr schlecht kristalliner Illit, HWB }>1^{\circ} 2 \Theta \text { (FEM, Abb. 6-17C) } \\
\text { - molare Elementverhältnisse aus der Formeleinheit }\end{array}$}} \\
\hline & & & & \\
\hline & $-\mathrm{Si} / \mathrm{Al} \approx 2,1$ & $\mathrm{Si} / \mathrm{Fe} \approx 19$ & $\mathrm{Si} / \mathrm{Mg} \approx 11$ & $\mathrm{Si} / \mathrm{K} \approx 5,7$ \\
\hline & \multicolumn{4}{|c|}{ - molare Elementverhältnisse aus der chemischen Analyse } \\
\hline & $-\mathrm{Si} / \mathrm{Al} \approx 2,41$ & $\mathrm{Si} / \mathrm{Fe} \approx 13,27$ & $\mathrm{Si} / \mathrm{Mg} \approx 18,32$ & $\mathrm{Si} / \mathrm{K} \approx 9,43$ \\
\hline mineralogische & - Illit & $55-6$ & Gew.- \% & \\
\hline \multirow[t]{5}{*}{ Zusammensetzung } & Quarz & $\approx 15$ & & \\
\hline & Chlorit/Kaolinit & $\approx 15$ & & \\
\hline & Albit & $<5$ & & \\
\hline & Mikroklin & $<5$ & & \\
\hline & Fe-Oxidhydrat & $<3$ & & \\
\hline
\end{tabular}

* nach Hower \& Mowatt (1966)

Illit OECD - Le Puy en Velay

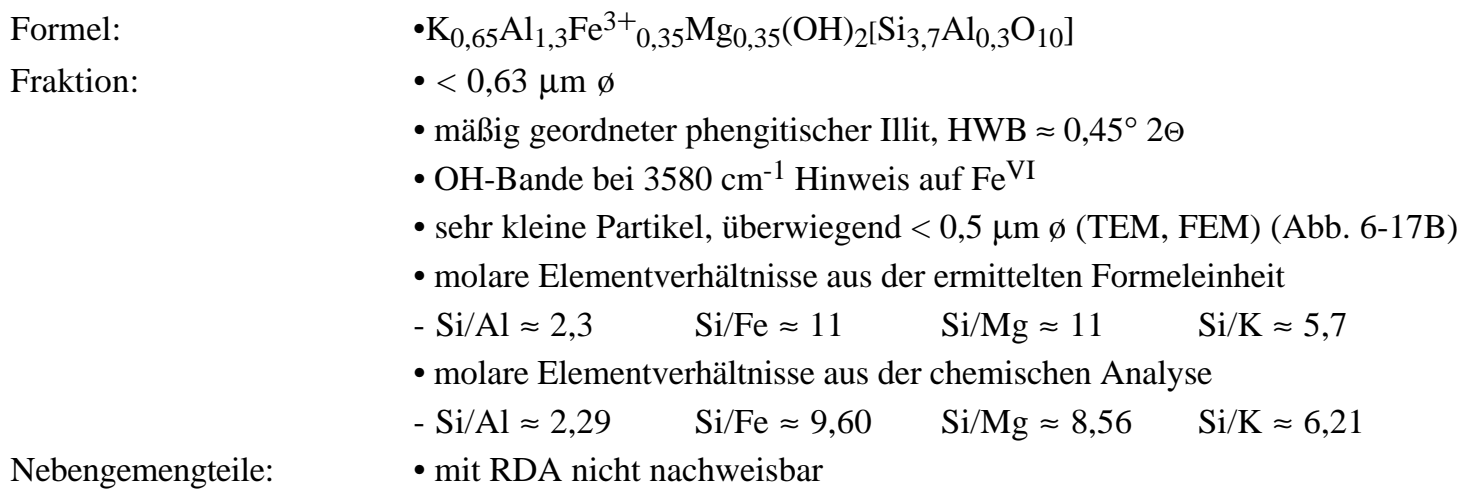

Montmorillonit H25 - Upton/Wyoming

$\begin{array}{ll}\text { Formel: } & \cdot \mathrm{Al}_{1,5} \mathrm{Fe}_{0,18} \mathrm{Mg}_{0,23}(\mathrm{OH})_{2}\left[\mathrm{Si}_{3,94} \mathrm{Al}_{0,06} \mathrm{O}_{10}\right] \mathrm{Na}_{0,34} \\ \text { Fraktion: } & -<0,63 \mu \mathrm{m} \emptyset \quad(\mathrm{FEM}, \mathrm{Abb} .6-10) \\ & \cdot \text { molare Elementverhältnisse aus der ermittelten Formeleinheit } \\ & -\mathrm{Si} / \mathrm{Al} \approx 2,5 \quad \mathrm{Si} / \mathrm{Fe} \approx 22 \quad \mathrm{Si} / \mathrm{Mg} \approx 17 \quad \mathrm{Si} / \mathrm{Na} \approx 12 \\ & \cdot \text { molare Elementverhältnisse aus der chemischen Analyse } \\ & -\mathrm{Si} / \mathrm{Al} \approx 2,44 \quad \mathrm{Si} / \mathrm{Fe} \approx 21,73 \quad \mathrm{Si} / \mathrm{Mg} \approx 17,00 \quad \mathrm{Si} / \mathrm{Na} \approx 13,95 \\ & - \text { mit RDA nicht nachweisbar }\end{array}$


Tab. 5-1: Fortsetzung

Serizit - Ausschlag/Zöbern

Formel:

- $\mathrm{KAl}_{1,6} \mathrm{Fe}^{3+}{ }_{0,1} \mathrm{Mg}_{0,3}(\mathrm{OH})_{2}\left[\mathrm{Si}_{3,3} \mathrm{Al}_{0,7} \mathrm{O}_{10}\right]$

Fraktion:

- $6,3-20 \mu \mathrm{m} \varnothing$

- sehr gut kristalliner phengitischer Serizit, $\mathrm{HWB}<0,2^{\circ} 2 \Theta$

- große schuppige, sehr dünne Plättchen bis zu $50 \mu \mathrm{m} \varnothing$

(Mikroskopie, FEM) (Abb. 6-17A)

- molare Elementverhältnisse aus der ermittelten Formeleinheit

- $\mathrm{Si} / \mathrm{Al} \approx 1,4 \quad \mathrm{Si} / \mathrm{Fe} \approx 33 \quad \mathrm{Si} / \mathrm{Mg} \approx 11 \quad \mathrm{Si} / \mathrm{K} \approx 3,3$

- molare Elementverhältnisse aus der chemischen Analyse

mineralogische

- $\mathrm{Si} / \mathrm{Al} \approx 2,77$

$\mathrm{Si} / \mathrm{Fe} \approx 31,26$

$\mathrm{Si} / \mathrm{Mg} \approx 18,55$

$\mathrm{Si} / \mathrm{K} \approx 6,53$

Zusammensetzung

- Serizit $\approx 70$ Gew. $\%$

Quarz $\approx 30$ Gew.- $\%$

Talk - Valle del Chisone

Formel:

Fraktion:

- $\mathrm{Mg}_{2,9} \mathrm{Fe}_{0,05}(\mathrm{OH})_{2}\left[\mathrm{Si}_{4} \mathrm{O}_{10}\right]$

- 2 - $63 \mu \mathrm{m} \emptyset$

- Talkpartikel zeigen lamellaren Habitus mit unregelmäßigen Konturen, eine Folge des Mahlprozesses (TEM, FEM) (Abb. 6-28)

- molare Elementverhältnisse aus der ermittelten Formeleinheit

- $\mathrm{Si} / \mathrm{Mg} \approx 1,4 \quad \mathrm{Si} / \mathrm{Fe} \approx 80$

- molare Elementverhältnisse aus der chemischen Analyse

- $\mathrm{Si} / \mathrm{Mg} \approx 1,40 \quad \mathrm{Si} / \mathrm{Fe} \approx 95,09 \quad \mathrm{Si} / \mathrm{Al} \approx 67,54$

Nebengemengteile:

- Spuren von Chlorit

\subsection{Lösungen}

Da der Schwerpunkt des Projektes auf der Untersuchung von Verwitterungsprozessen unter naturnahen Bedingungen lag, wurden als Säuren solche verwendet, die auch in der Natur vorkommen. So sind vor allem verdünnte Schwefelsäure $\left(\mathrm{H}_{2} \mathrm{SO}_{4}\right)$, aber auch Salpetersäure $\left(\mathrm{HNO}_{3}\right)$ und Salzsäure $(\mathrm{HCl})$ verwendet worden. Als Vertreter organischer Säuren diente Zitronensäure $\left(\mathrm{C}_{6} \mathrm{H}_{8} \mathrm{O}_{7}\right)$. Die Einstellung der unterschiedlichen $\mathrm{pH}$-Werte erfolgte nur mit der jeweiligen Säure. Um Effekte durch adsorbierbare Ionen, die nicht aus der Mineralauflösung stammen, weitgehend auszuschließen, wurde auf die Verwendung von Puffer-Lösungen oder die Einstellung einer definierten Ionenstärke verzichtet. Einige Experimente erfolgten auch mit $\mathrm{H}_{2} \mathrm{O}^{* * * *}$ und mit abgepreßter Poren-Lösung eines Bodenprofils im Solling.

Das zum Herstellen der Lösungen verwendete Wasser $\left(\mathrm{H}_{2} \mathrm{O}^{* * * *}\right)$ wurde mit einer Wasseraufbereitungsanlage (Seralpur Pro $90 \mathrm{CN}$ ) gewonnen. Die verwendeten Säuren entsprachen dem Reinheitsgrad suprapur $\left(\mathrm{H}_{2} \mathrm{SO}_{4}, \mathrm{C}_{6} \mathrm{H}_{8} \mathrm{O}_{7}\left(\right.\right.$ kristallin $\left.\left.\bullet 10 \mathrm{H}_{2} \mathrm{O}\right)\right)$ oder wurden durch Destillation in Suprasil-Quarz-Destillen $\left(\mathrm{HNO}_{3}\right)$ bzw. Teflon-Apparaturen $(\mathrm{HCl})$ in vergleichbarer Qualität gewonnen. 
Die Gewinnung der Poren-Lösung erfolgte durch Pressen des Feinbodenanteils $(\varnothing<2 \mathrm{~mm})$ mit einer Sedimentpresse (Böttcher et al. 1997). Die hierzu verwendete Apparatur ist in Abbildung A-3 schematisch dargestellt. Die chemische Charakterisierung der Porenlösung kann der Tabelle A-1 im Anhang entnommen werden. 


\section{Ergebnisse und Diskussion}

\subsection{Durchgeführte Experimente}

Viele der Versuche wurden im stärker sauren pH-Bereich ( $\mathrm{pH} 1$ und 2) durchgeführt. Solche $\mathrm{pH}-$ Werte liegen deutlich unter den $\mathrm{pH}-$ Werten in den meisten Böden und treten höchstens in speziellen Milieus wie Grubenwässern oder Deponiewässern auf. Die Auflösungsversuche wurden aber trotzdem im niedrigen $\mathrm{pH}$-Bereich durchgeführt, weil die Auflösungsprozesse unter diesen Bedingungen schneller ablaufen, wenn auch mit etwas anderen Reaktionsverläufen unter diesen stark sauren Bedingungen gerechnet werden muß.

Die Einstellung des „steady state” in den Durchfluß-Experimenten betrug meist mehr als 80 Tage. Für die Batch-Experimente wurden noch längere Versuchszeiträume gewählt, damit durch Akkumulation der gelösten Komponenten Konzentrationsbereiche erreicht werden konnten, bei denen mit der Bildung sekundärer Phasen gerechnet werden konnte. Bei den Durchfluß-Experimenten konnten unter den gewählten Bedingungen simultan mit dem Auflösungsprozeß ablaufende Reaktionen, wie Adsorption oder Präzipitatbildung weitgehend ausgeschlossen werden.

Für die im folgenden diskutierten Elemente wurden jeweils die wichtigen Gitterbausteine der Mineralproben ausgewählt. Eine Übersicht, welche Elemente für welches Mineral gemessen worden sind, findet sich in Tabelle 6-1.

Eine Übersicht über die durchgeführten Experimente ist in den Tabellen 6-2 und 6-3 gegeben. Weitere Daten wie Lösungsvolumina und eingewogene Menge Mineralpulver finden sich im Datenanhang unter den jeweiligen Versuchsbezeichnungen. Die in den Tabellen 6-2 und 6-3 genannten $\mathrm{pH}-\mathrm{Werte}$ entsprechen den zu Beginn der Experimente eingestellten Werten. Im Verlauf der Batch-Experimente stiegen diese zum Teil deutlich an. 
Tab. 6-1: Übersicht über die für die verschiedenen Mineralproben gemessenen Elemente.

\begin{tabular}{|l||cccccc|}
\hline & Kaolinit & Montmorillonit & Serizit & Illit H-35 & Illit OECD & Talk \\
\cline { 2 - 7 } $\mathrm{Al}$ & $\mathrm{X}$ & $\mathrm{X}$ & $\mathrm{X}$ & $\mathrm{X}$ & $\mathrm{X}$ & $\mathrm{X}$ \\
$\mathrm{Fe}$ & & $\mathrm{X}$ & $\mathrm{X}$ & $\mathrm{X}$ & $\mathrm{X}$ & $\mathrm{X}$ \\
$\mathrm{K}$ & & & $\mathrm{X}$ & $\mathrm{X}$ & $\mathrm{X}$ & \\
$\mathrm{Mg}$ & & $\mathrm{X}$ & $\mathrm{X}$ & $\mathrm{X}$ & $\mathrm{X}$ & $\mathrm{X}$ \\
$\mathrm{Na}$ & & $\mathrm{X}$ & & & & \\
$\mathrm{Si}$ & $\mathrm{X}$ & $\mathrm{X}$ & $\mathrm{X}$ & $\mathrm{X}$ & $\mathrm{X}$ & $\mathrm{X}$ \\
\hline
\end{tabular}

Tab. 6-2: Übersicht der durchgeführten Batch-Experimente. Die in () angeführten Zahlen stehen für die Versuchsbezeichnung unter der die zugehörigen Daten im Anhang aufgeführt sind.

\begin{tabular}{|c||cc|ccc|}
\hline Mineral & $\mathbf{H}_{2} \mathbf{S O}_{4}$ & $\mathbf{H N O}_{3}$ & $\mathbf{C}_{6} \mathbf{H}_{\mathbf{8}} \mathbf{O}_{7}$ & $\mathbf{H}_{2} \mathbf{O}^{* * * *}$ & $\begin{array}{c}\text { Poren- } \\
\text { Lösung-1 } \\
\end{array}$ \\
& $\mathrm{pH}$ & $\mathrm{pH}$ & $\mathrm{M}^{2}$ & $\mathrm{pH}^{3}$ & $\mathrm{pH}$ \\
\hline \hline Kaolinit & $1 ; 2 ; 3 ; 4$ & & 0,01 & $>4$ & 3,5 \\
$0,63-2 \mu \mathrm{m}$ & $(1 ; 7) ;(2 ; 8) ;(3) ;(4)$ & & $(18)$ & $(5)$ & $(47)$ \\
\hline Illit H35 & $1 ; 2 ; 3$ & $1 ; 2 ; 3$ & 0,01 & & 3,5 \\
$0,63-2 \mu \mathrm{m}$ & $(10) ;(11) ;(28)$ & $(31) ;(32) ;(33)$ & $(25)$ & & $(46)$ \\
\hline Illit OECD & $1 ; 2 ; 3$ & $1 ; 2 ; 3$ & 0,01 & & 3,5 \\
$<0,63 \mu \mathrm{m}$ & $(19) ;(22) ;(29)$ & $(34) ;(35) ;(36)$ & $(43)$ & & $(48)$ \\
\hline Montmorillonit & $1 ; 2 ; 3$ & $1 ; 2 ; 3$ & 0,01 & $>4$ & 3,5 \\
$<0,63 \mu \mathrm{m}$ & $(16) ;(17) ;(30)$ & $(37) ;(38) ;(39)$ & $(26)$ & $(49)$ & $(45)$ \\
\hline Serizit & $1 ; 2 ; 3$ & $1 ; 2 ; 3$ & 0,01 & $>4$ & 3,5 \\
$6,3-20 \mu \mathrm{m}$ & $(21) ;(24) ;(51)$ & $(52) ;(53) ;(54)$ & $(55)$ & $(50)$ & $(44)$ \\
\hline Talk OECD & $1 ; 2$ & & & & \\
$2-63 \mu \mathrm{m}$ & $(20) ;(23)$ & & & & \\
\hline
\end{tabular}

1 : abgepreßte Poren-Lösung eines Bodenprofils vom Solling

2 : M: $\mathrm{mol} / \mathrm{l} \rightarrow>0,01 \mathrm{~mol} / \mathrm{l}$ entspricht $\mathrm{pH} \approx 2,50$

3: wegen der geringen Ionenkonzentration in destilliertem Wasser ließ sich der $\mathrm{pH}-$ Wert nur schlecht messen. Ein $\mathrm{P}_{\mathrm{CO} 2}$ von $0,03 \mathrm{kPa}$ in der Luft entspricht einem $\mathrm{pH}$-Wert von 5,65 (Scheffer \& Schachtschabel 1992). 
Tab. 6-3: Übersicht der durchgeführten Durchfluß-Experimente. Die hinter den pH-Werten angeführten Ziffern stehen für die Versuchsbezeichnung unter der die zugehörigen Daten im Anhang aufgeführt sind.

\begin{tabular}{|c|c|c|c|c|c|c|c|c|}
\hline \multirow[t]{2}{*}{ Mineral } & \multicolumn{2}{|c|}{$\mathrm{H}_{2} \mathrm{SO}_{4}$} & \multicolumn{2}{|c|}{$\mathrm{HNO}_{3}$} & \multicolumn{2}{|c|}{$\overline{\mathrm{HCl}}$} & \multicolumn{2}{|c|}{$\mathrm{C}_{6} \mathrm{H}_{8} \mathrm{O}_{7}$} \\
\hline & $\mathrm{pH}$ & $\mathrm{g} / \mathrm{h}^{1}$ & $\mathrm{pH}$ & $\mathrm{g} / \mathrm{h}^{1}$ & $\mathrm{pH}$ & $\mathrm{g} / \mathrm{h}^{1}$ & $\mathrm{M}^{2}$ & $\mathrm{~g} / \mathrm{h}^{1}$ \\
\hline \multirow{6}{*}{$\begin{array}{l}\text { Kaolinit } \\
0,63-2 \mu \mathrm{m}\end{array}$} & 1 IV90(1) & 1 & $1 \mathrm{~V} 90$ & 0,4 & $1 \mathrm{VI} 90$ & 0,4 & $\mathbf{0 , 0 1} X(1)$ & 2 \\
\hline & 1 IV250(1) & 1 & $1 \mathrm{~V} 250(2)$ & 1 & 1 VI250(1) & 0,4 & $0,05 \times(2)$ & 2 \\
\hline & 2 IV90(2) & 0,3 & 2 VI250(2) & 1 & 2 VI250(2) & 1 & & \\
\hline & 2 IV250(2) & 0,3 & 3 VI250(3) & 1 & 3 VI250(3) & 1 & & \\
\hline & |2 IV250(4) & 1 & 3 VI250(4) & 1 & & & & \\
\hline & 2 II & 1 & & & & & & \\
\hline Illit H35 & $1 \mathrm{VII}(1)$ & 2 & & & & & & \\
\hline $0,63-2 \mu \mathrm{m}$ & $2 \mathrm{VII}(2)$ & 3 & & & & & & \\
\hline & $\mathbf{3} \mathrm{VII}(3)$ & 3 & & & & & & \\
\hline Illit OECD & $1 \mathrm{XII}(1)$ & 2 & & & & & & \\
\hline$<0,63 \mu \mathrm{m}$ & 2 XII(2) & 3 & & & & & & \\
\hline Montmorillonit & $1 \mathrm{VIII}(1)$ & 2 & & & & & $\mathbf{0 , 0 1}$ XIV & 2 \\
\hline$<0,63 \mu \mathrm{m}$ & 2 VIII(2) & 3 & & & & & & \\
\hline & 3 VIII(3) & 3 & & & & & & \\
\hline $\begin{array}{l}\text { Serizit } \\
6,3-20 \mu \mathrm{m}\end{array}$ & $\begin{array}{ll}1 & \mathrm{IX}(1) \\
2 & \mathrm{IX}(2)\end{array}$ & $\begin{array}{l}3 \\
3\end{array}$ & & & & & $\mathbf{0 , 0 1}$ X V & 3 \\
\hline $\begin{array}{l}\text { Talk OECD } \\
2-63 \mu \mathrm{m}\end{array}$ & $1 \mathrm{XI}$ & 3 & 1 (XIII) & 3 & & & & \\
\hline
\end{tabular}

1: Durchflußrate der Lösung durch den Reaktor

2: $\mathrm{M}: \mathrm{mol} / \mathrm{l}->0,01 \mathrm{~mol} / \mathrm{l}$ entspricht $\mathrm{pH} \approx 2,50 ; 0,05 \mathrm{~mol} / \mathrm{l}$ entpricht $\mathrm{pH} \approx 2,17$ 


\subsection{Kaolinit}

Kaolinite sind Zweischicht-Minerale, die aus einer Tetraeder- und einer Oktaederschicht mit $\mathrm{Si}^{4+}$ in den Tetraeder- und $\mathrm{Al}^{3+}$ in den Oktaederpositionen aufgebaut sind. Sie zeigen nur geringe chemische Varianz. Der für die Experimente ausgewählte Kaolinit ist gut kristallin mit hexagonalen Umrissen und scharfen Konturen an den Kanten. In Abbildung 6-1 sind diese morphologischen Eigenschaften beispielhaft in einer FEM- (Abb. 6-1A) und einer AFMAufnahme (Abb. 6-1B) gezeigt. In der FEM-Aufnahme ist als ein weiteres Merkmal die Zusammenlagerung von Mineralblättchen zu Stapelpaketen zu erkennen.

In Abbildung 6-2 sind exemplarisch die Reaktionsverläufe von drei Batch-Versuchen dargestellt, die bei unterschiedlichen $\mathrm{pH}$-Bedingungen aber sonst gleichen Startvorgaben durchgeführt wurden (Versuche 1, 2 und 5; Tab. A-5 und A-7).

Aus dem Kurvenverlauf ist zunächst ablesbar, daß sich der Auflösungsprozeß mit steigendem pH verlangsamt. Die Konzentrationen der zu einem bestimten Zeitpunkt in Lösung gegangenen Ionen sind bei höheren $\mathrm{pH}-$ Werten niedriger. Dies spiegelt sich auch deutlich wider in den Lösungsgeschwindigkeiten $\mathrm{R}_{\mathrm{Si}}$ und $\mathrm{R}_{\mathrm{Al}}$ (Tab. 6-4). Neben dem Auflösungsprozeß des Kaolinites muß noch mit anderen Prozessen gerechnet werden, da sich mit steigendem $\mathrm{pH}$ auch die $\mathrm{R}_{\mathrm{Si}} / \mathrm{R}_{\mathrm{Al}}$-Verhälnisse ändern, die etwa die Element-Molverhältnisse repräsentieren.

Tab. 6-4: Lösungsgeschwindigkeiten $\mathrm{R}_{S i}$ und $\mathrm{R}_{\mathrm{Al}}$ von Kaolinit in $\mathrm{H}_{2} \mathrm{SO}_{4}$-saurer Lösung bzw. in $\mathrm{H}_{2} \mathrm{O}^{* * * *}$ in Batch-Versuchen (50 mg Kaolinit / $100 \mathrm{ml}$ Lösung).

\begin{tabular}{ccccc}
\hline Versuch & $\mathrm{pH}$ & $\begin{array}{c}\mathrm{R}_{\mathrm{Si}} \\
{[\mathrm{mol} /(\mathrm{g} \bullet \mathrm{h})]}\end{array}$ & $\begin{array}{c}\mathrm{R}_{\mathrm{Al}} \\
{[\mathrm{mol} /(\mathrm{g} \bullet \mathrm{h})]}\end{array}$ & $\mathrm{R}_{\mathrm{Si}} / \mathrm{R}_{\mathrm{Al}}$ \\
1 & 1 & $1,1 \cdot 10^{-8}$ & $9,8 \bullet 10^{-9}$ & 1,1 \\
2 & 2 & $4,6 \bullet 10^{-9}$ & $3,3 \bullet 10^{-9}$ & 1,4 \\
5 & $\approx 4$ & $1,7 \bullet 10^{-9}$ & $2,3 \bullet 10^{-10}$ & 7,4 \\
\hline
\end{tabular}

Bei pH 1 liegt das Molverhältnis Si/Al in der Lösung bei $\approx 1$ (Abb. 6-2), die Auflösungsgeschwindigkeiten $\mathrm{R}_{\mathrm{Si}}$ und $\mathrm{R}_{\mathrm{Al}}$ sind annähernd gleich groß. Daraus folgt, daß sich der Kaolinit, mit einem Molverhältnis Si/Al im Festkörper von 1,06 (Tab. 5-1), kongruent unter diesen Bedingungen auflöst.

Bei pH 2 liegt im gesamten Versuchsverlauf die Al-Kozentration in der Lösung immer deutlich unter der der Si-Konzentration (Abb. 6-2). Sie spiegelt sich auch in den Lösungsgeschwindigkeiten wider, $\mathrm{R}_{\mathrm{Si}}>\mathrm{R}_{\mathrm{Al}}$. Bei dem Versuch bei $\mathrm{pH} \approx 4$ tritt dieser Effekt noch stärker auf, hier steigt der Wert $\mathrm{R}_{\mathrm{Si}} / \mathrm{R}_{\mathrm{Al}}$ auf fast $10 \mathrm{an}$. Solche Reaktionsabläufe müßten 

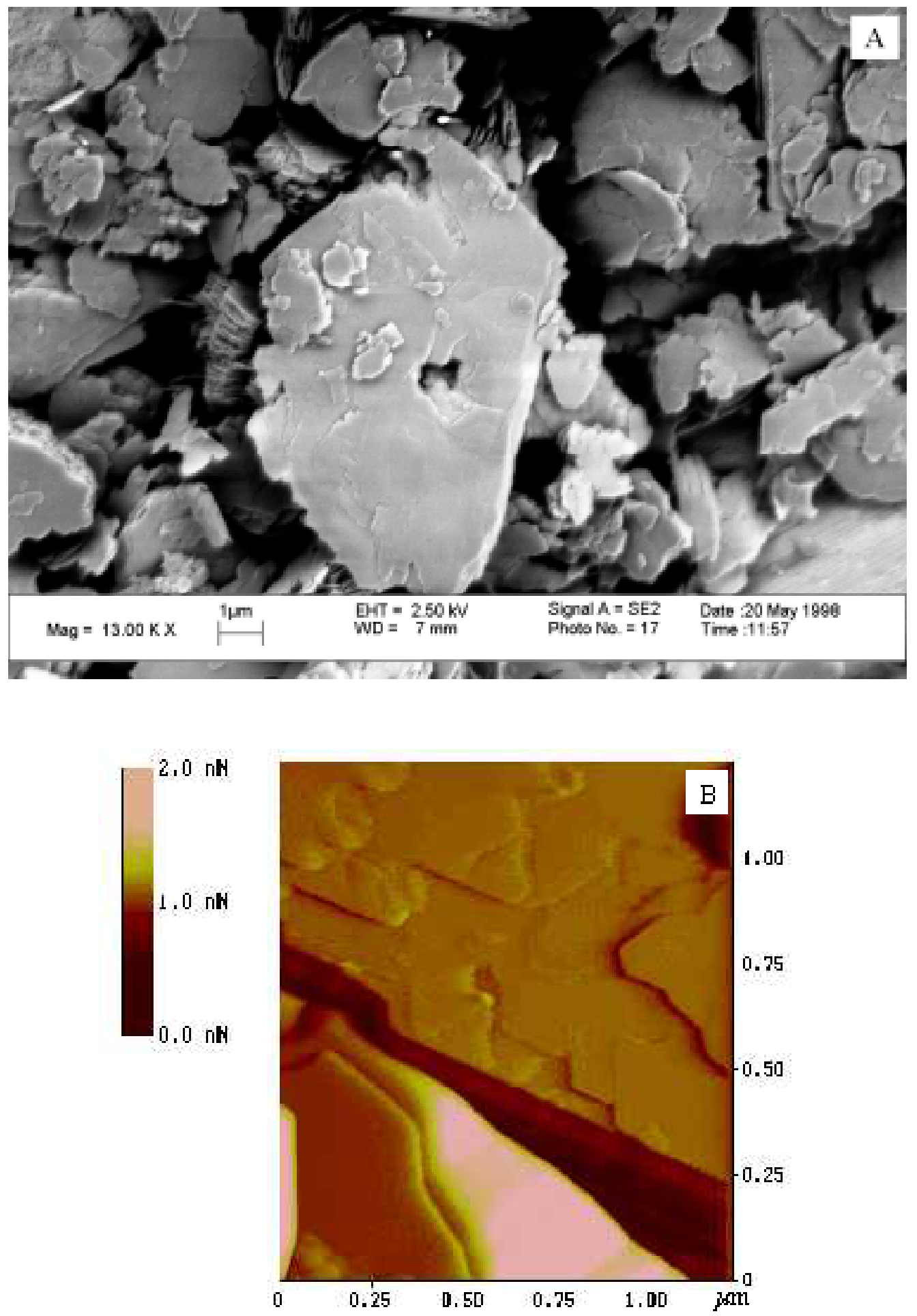

Abb. 6-1: FEM- (A) und AFM (B)-Aufnahmen des unbehandelten Kaolinites. In der AFMAufnahme ist die Größe der Partikel in $x$ - und y-Richtung in $\mu m$ aufgetragen. Die Höhenunterschiede der Oberflächentopographie sind als Resultat aus der Wechselwirkung der abrasternden Sonde mit der Oberfläche in nano Newton (nN) angegeben. 
als inkongruente Auflösungsprozesse beschrieben werden. Es wurde aber bereits in Kapitel 2.3 darauf hingewiesen, daß eine nichtstöchiometrische Auflösung durch simultan mit der kongruenten Auflösung ablaufende Reaktionen erklärt werden kann. Auf der Basis der Ergebnisse scheinen folgende Reaktionen simultan abzulaufen:

kongruente Auflösung des Kaolinits gekoppelt mit fast gleichzeitigem Entfernen der in Lösung gegangenen $\mathrm{Al}^{3+-}$ Ionen durch Adsorption an den Partikeloberflächen. Auf diese simultan ablaufenden Reaktionen hat bereits Heydemann (1966) hingewiesen. Das von ihr untersuchte Fireclay-Mineral zeigte bei $\mathrm{pH} 3$ in $\mathrm{H}_{2} \mathrm{SO}_{4}$-saurer Lösung ein ähnliches Verhalten wie der hier untersuchte Kaolinit von Zettlitz, während der von ihr untersuchte Kaolinit H-7 unter den gleichen Bedingungen kongruent - ohne merkliche Adsorptionseffekte - in Lösung ging.

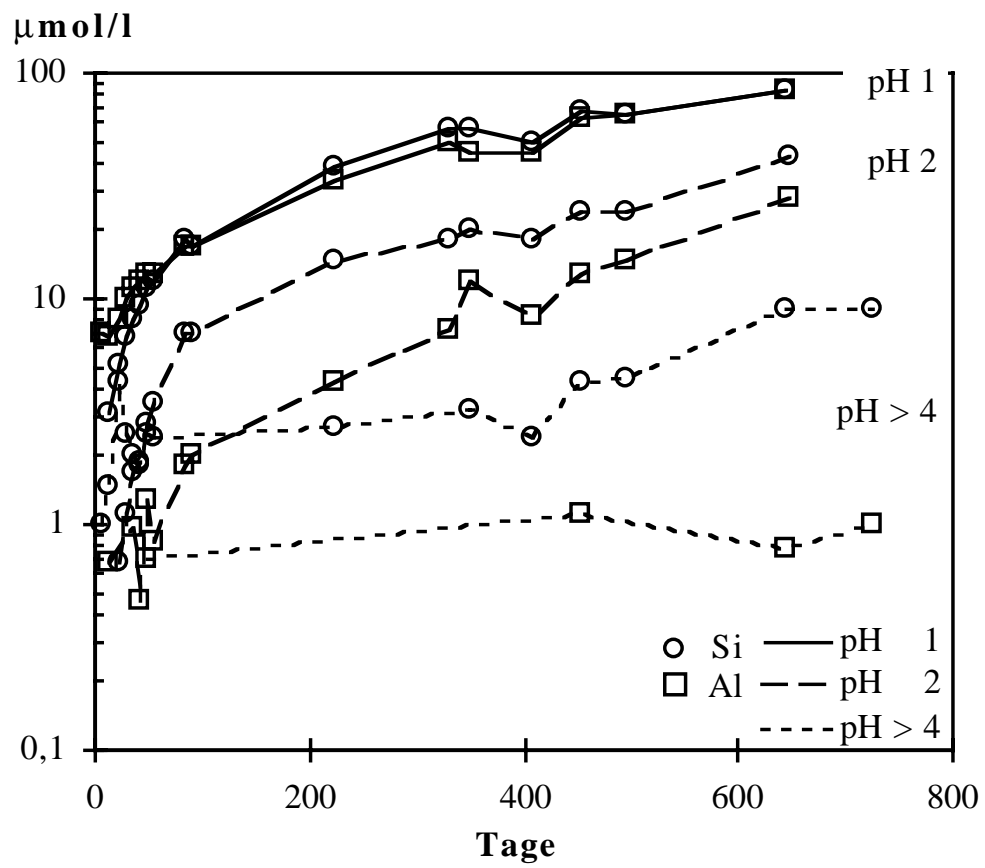

Abb. 6-2: Batch-Versuche mit $50 \mathrm{mg}$ Kaolinit in $100 \mathrm{ml}$ Lösung; Versuche 1 (pH $1 \mathrm{H}_{2} \mathrm{SO}_{4}$ ), 2 $\left(\mathrm{pH}_{2} \mathrm{H}_{2} \mathrm{SO}_{4}\right)$ und $5\left(\mathrm{pH}>4 \mathrm{H}_{2} \mathrm{O}^{* * * * *}\right)$.

Ähnliche Kurvenverläufe wurden bei Versuchen mit erhöhtem Festkörper- / Lösungs-Verhältnis beobachtet (Abb. 6-3). Insgesamt liegen hier die Si- und Al-Konzentrationen bei vergleichbaren Reaktionszeiten höher, die Lösungsgeschwindigkeiten $\mathrm{R}_{\mathrm{Si}}$ und $\mathrm{R}_{\mathrm{Al}}$ sind jedoch vergleichbar. Bemerkenswert ist bei dem Versuch 7 bei pH 1, daß nach etwa 300 Tagen Versuchsdauer die Al-Konzentration langsamer ansteigt. Die zunächst beobachtete kongruente Auflösung scheint nun von einem komplizierteren Prozeß abgelöst zu werden. Möglicherweise findet bei erhöhter Al-Konzentration in der Lösung auch unter diesen sauren Bedingungen Adsorption statt. 


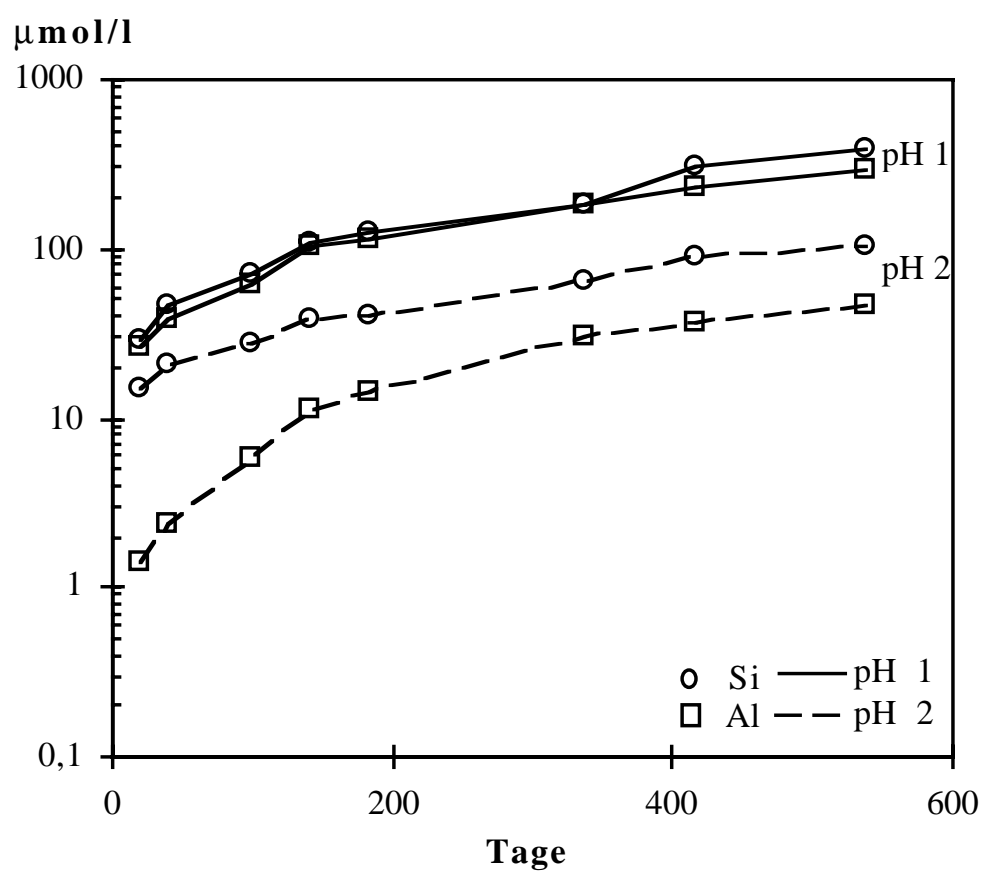

Abb. 6-3: Batch-Versuche mit $150 \mathrm{mg}$ Kaolinit in $100 \mathrm{ml} \mathrm{H}_{2} \mathrm{SO}_{4}$-saurer Lösung; Versuche 7 (pH 1) und 8 ( $\mathrm{pH} 2$ ).

Die mit $\mathrm{H}_{2} \mathrm{SO}_{4}$-sauren Lösungen durchgeführten Durchfluß-Experimente lassen auf ähnliche pH-abhängige Reaktionsabläufe schließen (Tab. 6-5), wie sie in den entsprechenden BatchVersuchen beobachtet wurden. Auch bei den Durchfluß-Experimenten mit $\mathrm{H}_{2} \mathrm{SO}_{4}$-sauren Lösungen geht der Kaolinit bei pH 1 kongruent in Lösung, während bei pH 2 mit simultan erfolgenden Adsorptionsprozessen gerechnet werden muß.

Tab. 6-5: Lösungsgeschwindigkeiten $R_{S i}$ und $R_{A l}$ sowie Angabe der Si- bzw. AlKonzentrationen im ,steady state” in Durchflu $\beta$-Experimenten mit Kaolinit in $\mathrm{H}_{2} \mathrm{SO}_{4}$-sauren Lösungen (0,75 g Kaolinit / $250 \mathrm{ml}$ Lösung, Durchflußgeschwindigkeit $\approx 1 \mathrm{~g} / \mathrm{h})$.

\begin{tabular}{ccccccc}
\hline Versuch & $\mathrm{pH}$ & $\begin{array}{c}\mathrm{R}_{\mathrm{Si}} \\
{[\mathrm{mol} /(\mathrm{g} \bullet \mathrm{h})]}\end{array}$ & $\begin{array}{c}\mathrm{R}_{\mathrm{Al}} \\
{[\mathrm{mol} /(\mathrm{g} \bullet \mathrm{h})]}\end{array}$ & $\mathrm{Si} / \mathrm{Al}$ & $\mathrm{Si}[\mu \mathrm{mol} / \mathrm{l}]$ & $\mathrm{Al}[\mu \mathrm{mol} / \mathrm{l}]$ \\
$\mathrm{IV}-250(1)$ & 1 & $6,6 \bullet 10^{-8}$ & $6,5 \bullet 10^{-8}$ & $\approx 1$ & 51 & 50 \\
$\mathrm{IV}-250(4)$ & 2 & $4,2 \cdot 10^{-8}$ & $1,9 \bullet 10^{-8}$ & 2,3 & 27 & 12 \\
\hline
\end{tabular}

Bemerkenwert ist, daß die Lösungsgeschwindigkeiten in den Durchfluß-Experimenten mit $\mathrm{H}_{2} \mathrm{SO}_{4}$-sauren Lösungen höher liegen als bei den entsprechenden Batch-Versuchen. Dies gilt auch für die Versuche mit Zitronensäure (Versuche 18, X(1), X(2)). Dieser Trend wird nicht auf das unterschiedliche Festkörper-/Lösungs-Verhältnis in den Versuchen zurückzuführen sein, sondern auf die unterschiedliche Art der Suspendierung der Partikel in den Experimenten. 
So wird im Fall der Batch-Versuche der Reaktor 30 mal pro Minute über Kopf gedreht, während in den Durchfluß-Experimenten die Rührgeschwindigkeiten in den Reaktoren bei 250 Umdrehungen pro Minute liegen. Durch die stärkere mechanische Beanspruchung der suspendierten Partikel in den Durchfluß-Reaktoren werden wahrscheinlich die teilweise in Form von Stapelpaketen vorliegenden Partikel (Abb. 6-1A) weiter aufbereitet, die reaktive Oberfläche dadurch vergrößert. (vgl. Kap. 4.2).

Bei den Durchfluß-Experimenten mit verdünnter $\mathrm{HNO}_{3}$ und $\mathrm{HCl}$ ist zwar auch eine deutliche Abhängigkeit des Auflösungsprozesses vom $\mathrm{pH}$-Wert erkennbar, aber schon bei $\mathrm{pH} 1$ beobachtet man in diesen Systemen nichtstöchiometrische Auflösung (Tab. 6-6).

Tab. 6-6: Lösungsgeschwindigkeiten $R_{S i}$ und $R_{A l}$ sowie Angabe der Si- und AlKonzentrationen im ,steady state” in Durchflu $\beta$-Experimenten mit Kaolinit in $\mathrm{HNO}_{3}$ - und HCl-sauren Lösungen.

\begin{tabular}{cccccccc}
\hline Versuch & Säure & $\mathrm{pH}$ & $\begin{array}{c}\mathrm{R}_{\mathrm{Si}} \\
{[\mathrm{mol} /(\mathrm{g} \bullet \mathrm{h})]}\end{array}$ & $\begin{array}{c}\mathrm{R}_{\mathrm{Al}} \\
{[\mathrm{mol} /(\mathrm{g} \bullet \mathrm{h})]}\end{array}$ & $\mathrm{Si} / \mathrm{Al}$ & $\mathrm{Si}[\mu \mathrm{mol} / \mathrm{l}]$ & $\mathrm{Al}[\mu \mathrm{mol} / \mathrm{l}]$ \\
$\mathrm{V}-250(2)$ & $\mathrm{HNO}_{3}$ & 1 & $4,9 \cdot 10^{-8}$ & $2,7 \cdot 10^{-8}$ & 2,0 & 41 & 21 \\
$\mathrm{VI}-250(1)$ & $\mathrm{HCl}$ & 1 & $4,6 \cdot 10^{-8}$ & $2,9 \bullet 10^{-8}$ & 1,6 & 90 & 55 \\
$\mathrm{VI}-250(2)$ & $\mathrm{HCl}$ & 2 & $1,3 \cdot 10^{-8}$ & $7,3 \cdot 10^{-9}$ & 2,4 & 11 & 4,6 \\
$\mathrm{VI}-250(3)$ & $\mathrm{HCl}$ & 3 & $1,1 \cdot 10^{-8}$ & $2,910^{-9}$ & 2,9 & 7,2 & 2,5 \\
$\mathrm{VI}-250(4)$ & $\mathrm{HCl}$ & 4 & $7,9 \cdot 10^{-9}$ & $3,3 \cdot 10^{-9}$ & 2,1 & 5,3 & 2,5 \\
\hline
\end{tabular}

Unter identischen experimentellen Bedingungen jedoch unterschiedlichem Einsatz von SäureAnionen ist zu beobachten, daß die Auflösungsgeschwindigkeit $\mathrm{R}_{\mathrm{Si}}$ für $\mathrm{H}_{2} \mathrm{SO}_{4}$-saure Lösungen am größten ist, gefolgt von $\mathrm{HNO}_{3}$ - und $\mathrm{HCl}$-sauren Lösungen (Tab. 6-5 und 6-6).

Abbildung 6-4 gibt noch einmal einen Überblick über die Ergebnisse der verschiedenen Batchund Durchfluß-Experimente. Die aufgetragenen $\mathrm{pH}$-Werte repräsentieren die in den Lösungen gemessenen Werte, die teilweise von den ursprünglich in den Experimenten eingestellten Werten (Tab. 6-2 und 6-3) abweichen können. Bei den Batch-Versuchen sind die im Verlauf der Versuche akkumulierten Si- und Al-Konzentrationen dargestellt, bei den DurchflußExperimenten geben die Konzentrationsangaben die Situation im „steady state” wider. Dies erklärt die niedigeren Konzentrationen im Teildiagramm (b)-Durchfluß verglichen mit Teildiagramm (a)-Batch. In beiden Diagrammen deutlich erkennbar ist die Abhängigkeit der SiKonzentrationen vom pH-Wert der Lösungen, d.h. mit steigendem pH-Wert nimmt die SiKonzentration in der Lösung ab. Im Fall der Al-Konzentrationen wird diese Abhängigkeit vom 
pH noch verstärkt, da die Al-Konzentrationen in der Lösung ja in vielen Fällen durch simultan mit der Auflösung ablaufende Reaktionen zu niedrigeren Werten verändert werden.

(a)

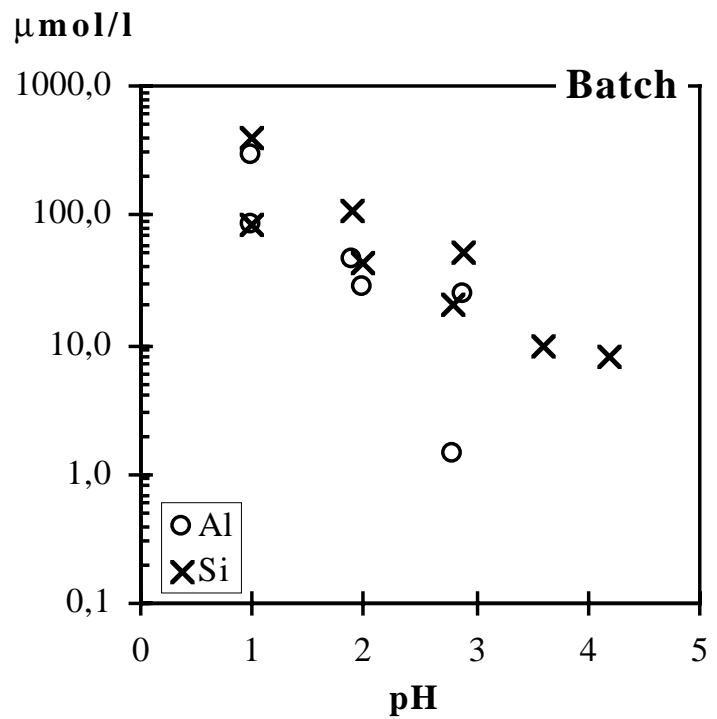

(b)

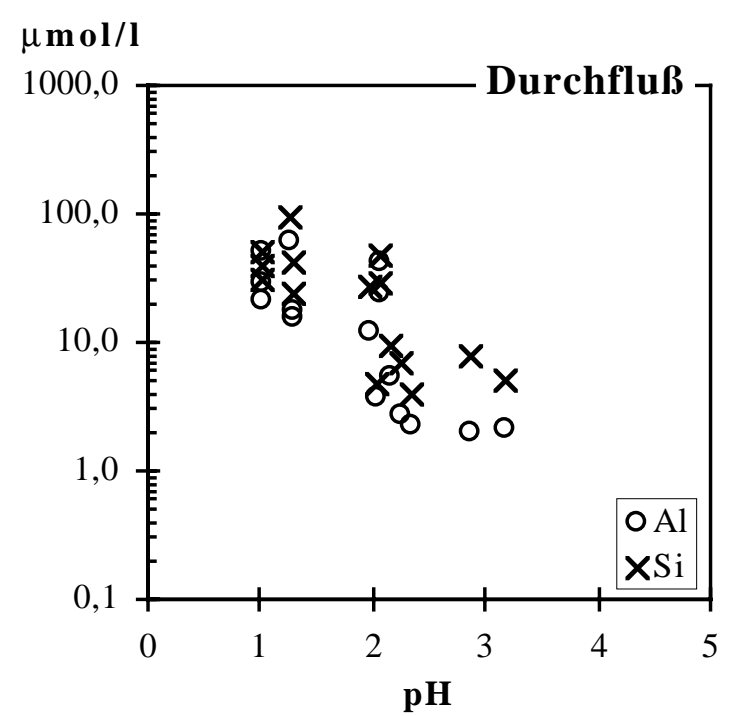

Abb. 6-4: Si- und Al-Konzentrationen in den verschiedenen Batch- und DurchflußExperimenten in Abhängigkeit vom $\mathrm{pH}$-Wert.

Batch -> im Verlauf der Versuche akkumulierte Konzentration

Durchflu $\beta$-> Konzentrationsangabe im , steady state”

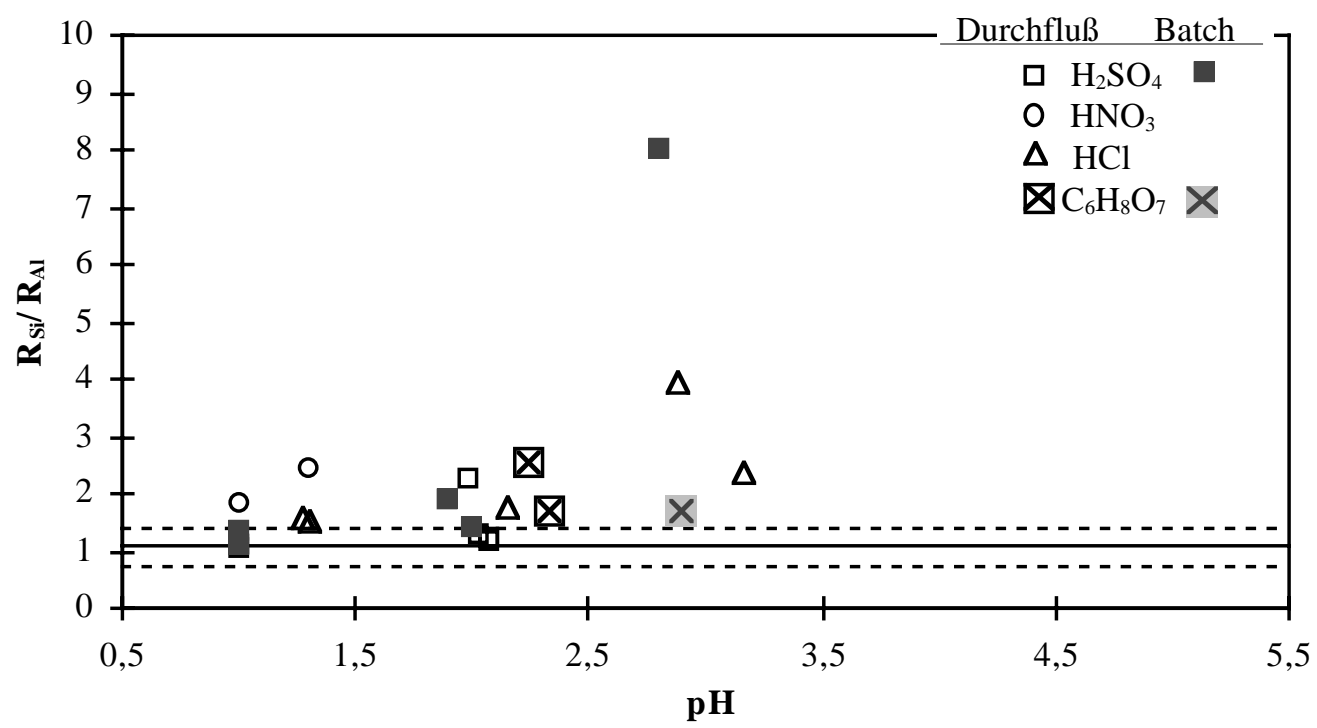

Abb. 6-5: $R_{S i} / R_{A l}$-Verhältnis in Abhängigkeit vom $p H$-Wert für die verschiedenen Lösungen in Batch- und Durchfluß-Experimenten. Die durchgezogene Linie entspricht dem stöchiometrischen Verhältnis im Festkörper. Die gestrichelten Linien repräsentieren den Fehlerbereich. 
Aus Abbildung 6-5 ist das unterschiedliche Verhalten von Kaolinit beim Auflösungsprozeß in Lösungen mit unterschiedlicher chemischer Zusammensetzung abzulesen: Kongruente Auflösung ohne simultan ablaufende Reaktionen ist eigentlich nur in $\mathrm{H}_{2} \mathrm{SO}_{4}$-saurer Lösung bei $\mathrm{pH} 1$ vorhanden, das $\mathrm{R}_{\mathrm{Si}} / \mathrm{R}_{\mathrm{Al}}$-Verhältnis liegt bei diesen Versuchen bei $\approx 1$.

Das variable Verhalten von Kaolinit-Mineralen beim Auflösungsprozeß beruht mit Sicherheit auf Unterschieden in den Oberflächenladungscharakteristika der Kaolinite, wird aber auch beeinflußt durch die unterschiedlichen Komplexierungseigenschaften der Komponenten in der Lösung. Die Oberflächenladungen der Kaolinite sind auf strukturell bedingte permanente Ladungen zurückzuführen, die $\mathrm{pH}$ unabhängig sind. Sie werden verursacht durch isomorphen Ersatz u.a. von $\mathrm{Si}^{4+}$ durch $\mathrm{Al}^{3+}$ oder $\mathrm{Fe}^{3+}$ in den Tetraederschichten sowie entsprechende geringfügige Substitutionen in der Oktaederschicht. Daraus resultieren negative Ladungen. Nur in Lösungen mit sehr geringer Ionenstärke werden diese für den Ionenaustausch verantwortlichen Plätze für Adsorptionsprozesse in Lösung gegangener $\mathrm{Al}^{3+}$-Ionen zur Verfügung stehen (Huertas et al. 1998). Die pH abhängige Ladung entsteht durch die Komplexierung der Oberfläche als Folge der Wechselwirkung mit der Lösung (Schroth \& Sposito 1997, Ward \& Brady 1998) und kann negativ oder positiv sein. Der dazwischen liegende Ladungsnullpunkt wird als ,point of zero charge” (PZC) bezeichnet.

Die Auflösung des Kaolinites erfolgt überwiegend durch Abbau über die Kanten, was dem in Kapitel 2 theoretisch beschriebenen Prozeß entspricht. Dabei kommt es zu Rundungseffekten an den Kanten (Abb. 6-6A-C). Dies ist schwach erkennbar nach Versuchsdauern von 28 Tagen bei $\mathrm{pH} 3$, deutlicher aber nach längeren Versuchszeiten und niedrigeren $\mathrm{pH}-$ Werten in der Lösung. Nach einer Reaktionszeit von 336 Tagen läßt sich bei dem Batch-Versuch mit $\mathrm{H}_{2} \mathrm{SO}_{4}$-saurer Lösung bei pH 2 außerdem eine Abnahme der Partikelgröße beobachten (Abb. 6-6C). Nach Vergleich mit dem Ausgangszustand (Abb. 6-1) kann nicht entschieden werden, ob dieser Effekt allein auf den Auflösungsprozeß zurückzuführen ist oder gekoppelt ist mit gewisser mechanischer Zerkleinerung der Kaolinitpartikel im Verlauf des Experimentes. Als weiterer Effekt ist eine zunehmende Bildung von Stufen und Rauhigkeiten auf den Partikeloberflächen zu beobachten. Als Beispiel dient hier Material aus einem Batch-Versuch nach 56 Tagen in $\mathrm{H}_{2} \mathrm{SO}_{4}$-saurer Lösung mit pH 1 (Abb. 6-6D). 
Ergebnisse und Diskussion
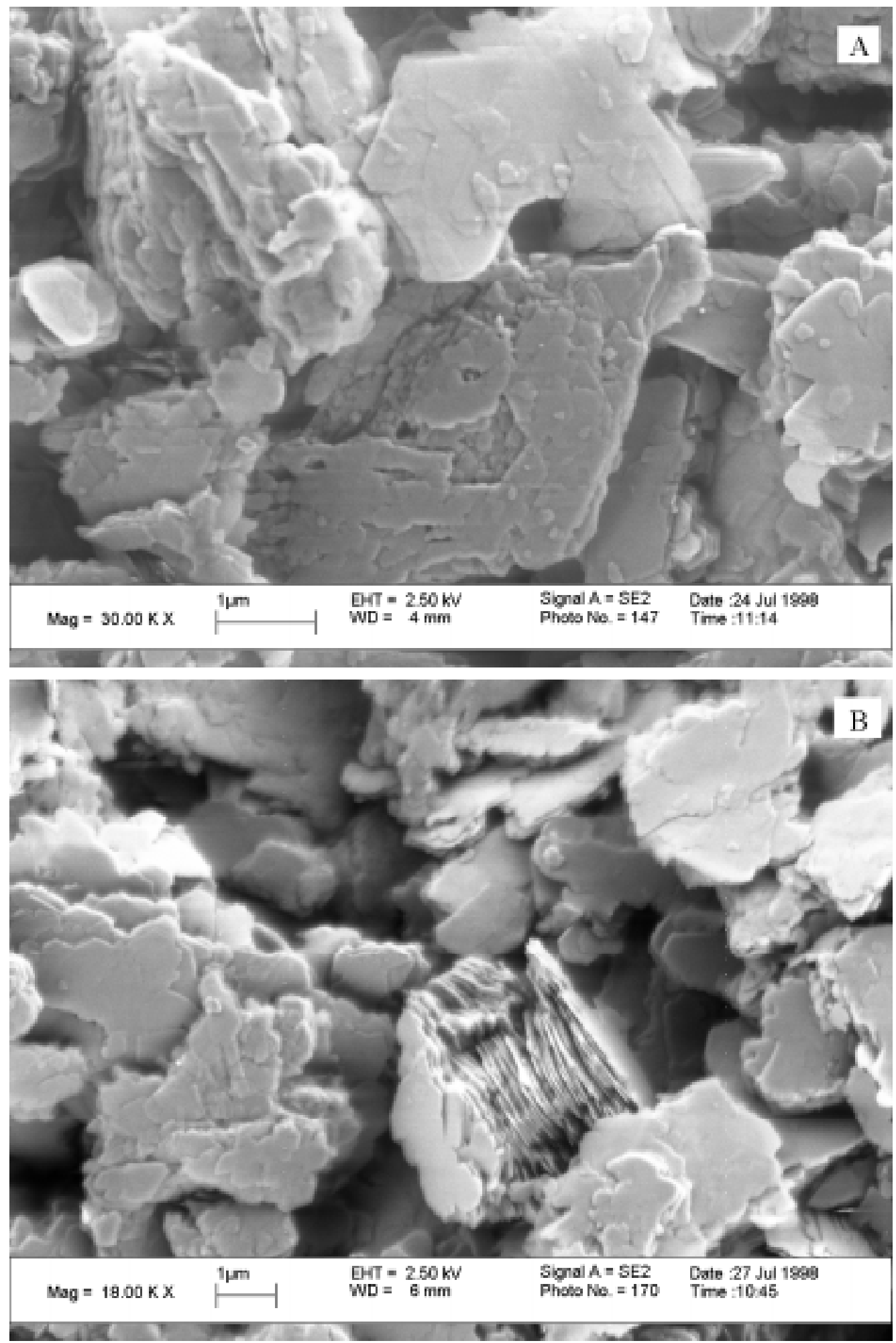

Abb. 6-6: Beschreibung siehe nächste Seite 

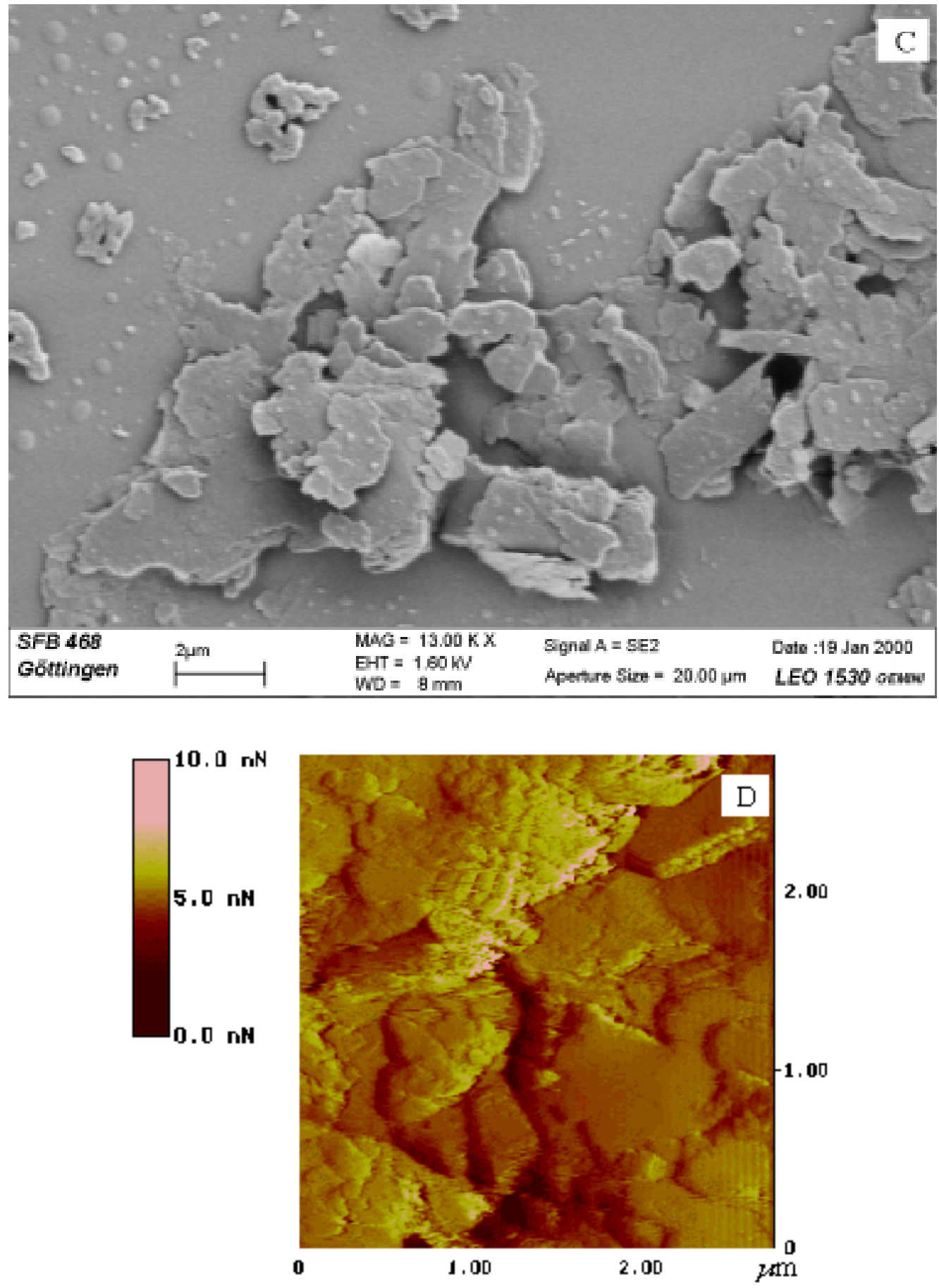

Abb. 6-6: FEM- (A-C) und AFM-Aufnahmen (D) des behandelten Kaolinites. A: BatchExperiment mit $\mathrm{H}_{2} \mathrm{SO}_{4}$ bei pH 3 nach 28 Tagen; B: Batch-Experiment mit $\mathrm{H}_{2} \mathrm{SO}_{4}$ bei pH 2 nach 91 Tagen; $C$ : Batch-Experiment mit $\mathrm{H}_{2} \mathrm{SO}_{4}$ bei pH 2 nach 336 Tagen; D: BatchExperiment mit $\mathrm{H}_{2} \mathrm{SO}_{4}$ bei pH 1 nach 56 Tagen. In der AFM-Aufnahme ist die Größe der Partikel in $x$ - und y-Richtung in $\mu m$ aufgetragen. Die Höhenunterschiede der Oberflächentopographie sind als Resultat aus der Wechselwirkung der abrasternden Sonde mit der Oberfläche in nano Newton (nN) angegeben. 
Ein Vergleich der hier ermittelten Ergebnisse mit Daten aus der Literatur erwies sich als schwierig, da nur wenige Experimentatoren ihre Versuche in verdünnten Lösungen ähnlicher chemischer Zusammensetzung und geringer Ionenstärke durchgeführt haben.

Huang \& Keller (1971) berichten über Batch-Versuche mit Kaoliniten aus Georgia und Keokuk in Wasser bei pH 4,2. Si/Al-Molverhältnisse in der Lösung mit Werten > 10 deuten auf einen ähnlichen Reaktionsablauf wie bei dem Versuch 5 (Tab. 6-4) hin, also möglichereise kongruente Auflösung gekoppelt mit starken Adsorptionseffekten. Bei Verwendung von $\mathrm{C}_{6} \mathrm{H}_{8} \mathrm{O}_{7}$ beobachteten sie im pH-Bereich 2 - 3 kongruente Auflösung der Kaolinite und führten dies auf die komplexierenden Eigenschaften von $\mathrm{C}_{6} \mathrm{H}_{8} \mathrm{O}_{7}$ in bezug auf gelöste Al-Ionen zurück, also auf einen ligandenkontrollierten Prozeß. Bei eigenen Untersuchungen mit $\mathrm{C}_{6} \mathrm{H}_{8} \mathrm{O}_{7}$ (Versuche 18, X(1) und X(2)) lagen die Si/Al-Molverhältnisse in der Lösung > 1,5 (Abb. 6-5).

Ganor et al. (1995) arbeiteten mit verdünnter $\mathrm{HClO}_{4}$, einem Medium, das in der Natur kaum vorkommt. Sie fanden bei ihren Durchfluß-Experimenten mit Kaolinit aus Georgia sogar noch bei pH 4,2 nach einer Versuchszeit von 29 Stunden annähernd kongruente Auflösung. Das Molverhältnis Si/Al in der Lösung lag bei $\approx 1,2$. Möglicherweise ist dies ein Hinweis auf die komplexierende Wirkung der $\mathrm{ClO}_{4}^{-}$-Anionen auf die in Lösung gegangenen $\mathrm{Al}^{3+}{ }^{3+}$ Ionen.

Viele in der Literatur beschriebenen Auflösungsuntersuchungen wurden in Systemen mit erhöhtem Elektrolytgehalt durchgeführt. Unter diesen Bedingungen sind die Adsorptionseffekte von gelösten Al-Ionen an der Oberfläche der Kaolinitpartikel natürlich stark eingeschränkt, die Ergebnisse also nur bedingt vergleichbar mit den hier vorgestellten Daten.

Carroll-Webb \& Walther (1988) verwendeten in ihren Batch-Versuchen im sauren Bereich Puffer $(\mathrm{KCl}+\mathrm{HCl}, \mathrm{KHP}$ thalate $+\mathrm{HCl})$ unterschiedlicher Ionenstärke $(0,005-0,05 \mathrm{Mol} / \mathrm{l})$. Bei einem pH-Wert von 1 beobachteten sie kongruente Auflösung des Kaolinites von Georgia. Mit steigendem $\mathrm{pH}$ nahm die Lösungsgeschwindigkeit $\mathrm{R}_{\mathrm{Si}}$ und $\mathrm{R}_{\mathrm{Al}}$ ab. Die Werte für das $\mathrm{R}_{\mathrm{Si}} / \mathrm{R}_{\mathrm{Al}}{ }^{-}$ Verhältnis stiegen an, was ein Hinweis auf nichtstöchiometrische Auflösung ist.

Wieland \& Stumm (1992) benutzten für ihre Batch-Experimente ( $\leq 15$ Tage) Lösungen mit konstantem Elektrolytzusatz von $0,1 \mathrm{M} \mathrm{NaNO}_{3} / 1$. In $\mathrm{HNO}_{3}$-sauren Lösungen im pH-Bereich 2 - 5 lösten sich die Kaolinite nichtstöchiometrisch auf. Die bei protonen-gesteuerten Auflösungsprozessen zum Teil gegenüber den $\mathrm{R}_{\mathrm{Al}}$-Werten höheren $\mathrm{R}_{\mathrm{Si}}$-Werte versuchten sie durch Adsorption von $\mathrm{Al}$ an den Kaolinitpartikeln und die Bildung sekundärer Al-reicher Präzipitate zu erklären. Zusatz von Oxalat zu den Lösungen unterband diese Reaktionen im pHBereich 2 - 4, der Kaolinit löste sich unter diesen überwiegend ligandenkontrollierten Bedingungen kongruent auf. Die Lösungsgeschwindigkeiten lagen unter diesen Bedingungen höher als bei dem rein protonengesteuerten Abbau (Tab. 6-7). 
Auch Sutheimer et al. (1999) untersuchten das Auflösungsverhalten von Kaoliniten in BatchExperimenten unter Verwendung von Elektrolytzusatz $\left(0,01 \mathrm{M} \mathrm{NaNO}_{3}\right)$. Der parabolische Verlauf der Kurven zu Beginn der Versuche deutet auf Effekte hin, die von der Auflösung sehr kleiner reaktiver Partikel und/oder von amorphem Material herrühren und bei den kurzen Versuchszeiten ( $\leq 13$ Tage) die Endergebnisse durchaus beeinflussen können. Bei den ligandenkontrollierten Versuchen mit Oxalat-Zusatz beobachteten sie bei pH 3 zum Teil höhere Konzentrationen von $\mathrm{Al}$ in der Lösung im Vergleich zu Si, also Si/Al-Molverhältnisse in der Lösung von 0,7 bis 0,85 (Tab. 6-7). Von Sutheimer et al. (1999) wird dies als annähernd kongruente Auflösung beschrieben. Unter $\mathrm{HNO}_{3}$-sauren, protonengesteuerten Bedingungen lagen dagegen die Al-Konzentrationen in der Lösung deutlich unter denen von Si (also nichtstöchiometrische Auflösung). Im Gegensatz zu Wieland \& Stumm (1992) ist bei Sutheimer et al. (1999) eine Zunahme der Lösungsgeschwindigkeit durch Ligandenzugabe nicht immer erkennbar.

Tab. 6-7: Auflösungsraten für Si und Al bei protonen- und/oder ligandengesteuerten Auflösungsprozessen von Kaoliniten bei pH 3 und $20-25^{\circ} \mathrm{C}$ in Abhängigkeit von der Lösungszusammensetzung (Daten aus der Literatur). k.A.: keine Angabe, ${ }^{1} \mathrm{Al}<<\mathrm{Si}[\mathrm{nmol} / \mathrm{l}]$

\begin{tabular}{|c|c|c|c|c|c|}
\hline $\begin{array}{l}\text { Literatur } \\
\text { (Probe) }\end{array}$ & Säure & $\begin{array}{c}\text { Elektrolyt } \\
{[\mathrm{mol} / \mathrm{l}]}\end{array}$ & $\begin{array}{l}\text { Ligand } \\
{[\mathrm{mol} / \mathrm{l}]} \\
\end{array}$ & $\begin{array}{c}\mathrm{R}_{\mathrm{Si}} \\
{\left[\mathrm{nmol} /\left(\mathrm{m}^{2} \bullet \mathrm{h}\right)\right]} \\
\end{array}$ & $\begin{array}{c}\mathrm{R}_{\mathrm{Al}} \\
{\left[\mathrm{nmol} /\left(\mathrm{m}^{2} \bullet \mathrm{h}\right)\right]}\end{array}$ \\
\hline \multirow[t]{2}{*}{ Ganor et al. (1995) } & $\mathrm{HClO}_{4}$ & --- & --- & 2,4 & 3,1 \\
\hline & $\mathrm{HClO}_{4}$ & --- & --- & 2,4 & 2,6 \\
\hline $\begin{array}{l}\text { Carrol-Webb \& } \\
\text { Walther (1988) }\end{array}$ & $\mathrm{HCl}$ & $\mathrm{KCl}: 0,005$ & --- & 0,47 & 0,34 \\
\hline \multirow{5}{*}{$\begin{array}{l}\text { Wieland \& Stumm } \\
\text { (1992) }\end{array}$} & $\mathrm{HNO}_{3}$ & $\mathrm{NaNO}_{3}: 0,1$ & --- & 2,63 & 2,71 \\
\hline & $\mathrm{HNO}_{3}$ & $\mathrm{NaNO}_{3}: 0,1$ & --- & 3,34 & 1,78 \\
\hline & $\mathrm{HNO}_{3}$ & $\mathrm{NaNO}_{3}: 0,1$ & Oxalat: 0,001 & 3,84 & 3,83 \\
\hline & $\mathrm{HNO}_{3}$ & $\mathrm{NaNO}_{3}: 0,1$ & Oxalat: 0,001 & 4,22 & 4,18 \\
\hline & $\mathrm{HNO}_{3}$ & $\mathrm{NaNO}_{3}: 0,1$ & Oxalat: 0,001 & 4,21 & 4,09 \\
\hline \multicolumn{6}{|c|}{ Sutheimer et al. (1999) } \\
\hline \multirow[t]{2}{*}{ Kaol 1} & $\mathrm{HNO}_{3}$ & $\mathrm{NaNO}_{3}: 0,01$ & --- & 1,16 & k.A. 1 \\
\hline & $\mathrm{HNO}_{3}$ & $\mathrm{NaNO}_{3}: 0,01$ & Oxalat: 0,001 & 0,96 & 1,31 \\
\hline \multirow[t]{2}{*}{ Kaol 2} & $\mathrm{HNO}_{3}$ & $\mathrm{NaNO}_{3}: 0,01$ & --- & 0,86 & k.A. ${ }^{1}$ \\
\hline & $\mathrm{HNO}_{3}$ & $\mathrm{NaNO}_{3}: 0,01$ & Oxalat: 0,001 & 2,27 & 2,66 \\
\hline
\end{tabular}

In der Literatur werden die Raten $\mathrm{R}_{\mathrm{Si}}$ und $\mathrm{R}_{\mathrm{Al}}$ bei den Auflösungsversuchen mit Kaolinit im allgemeinen in $\left[\mathrm{nmol} / \mathrm{m}^{2} \mathrm{~h}\right]$ angegeben. In dieser Arbeit wird die Angabe für die Lösungsgeschwindigkeit in $[\mathrm{mol} /(\mathrm{g} \bullet \mathrm{h})]$ bevorzugt. In dieser Form lassen sich auch Untersuchungsergebnisse von Smektit- und Illit-Abbau einander gegenüberstellen (vgl. Kap. 2). 
Eine Möglichkeit, die verschiedenen Untersuchungsergebnisse trotzdem miteinander vergleichen zu können, bietet die Reaktionsordnung (vgl. Kap. 2.3). In Abbildung 6-7 ist diese Reaktionsordnung n in Form der Steigung sowohl für die durchgeführten Durchfluß- (Abb. 67a) als auch für die Batch-Experimente (Abb. 6-7b) abzulesen.

Bei den Durchfluß-Experimenten lassen sich innerhalb des Fehlerbereiches identische Reaktionsordnungen für die mit $\mathrm{H}_{2} \mathrm{SO}_{4}$ bzw. $\mathrm{HCl}$ durchgeführten Versuche ablesen. Die Ausgleichsgerade für die Experimente mit $\mathrm{HCl}$ ist aber mit nur wenig geringeren Auflösungsraten parallel verschoben. Die Reaktionsordnung bei den Batch-Experimenten mit $\mathrm{H}_{2} \mathrm{SO}_{4}$ ist identisch mit der in den Durchfluß-Experimenten ermittelten Reaktionsordnung.
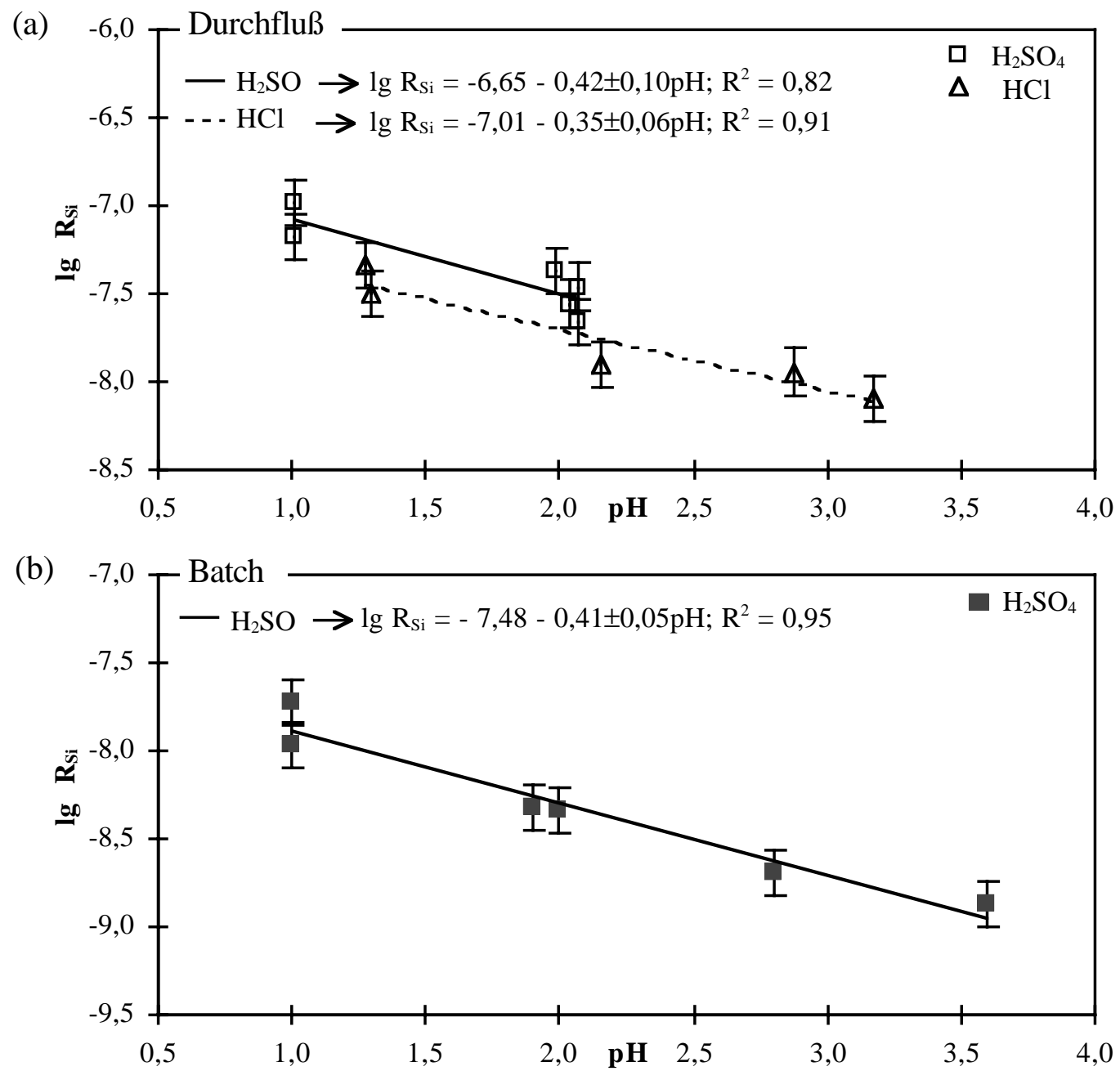

Abb. 6-7: Logarithmus der Auflösungsrate von $S i\left(R_{S i}\right)$ in Abhängigkeit vom pH-Wert für Batch- und Durchfluß-Experimente zur Bestimmung der Reaktionsordnung. Die Reaktionsordnung $n$ ergibt sich jeweils aus der Steigung der Geraden. 
In Tabelle 6-8 sind einige Reaktionsordnungen protonengesteuerter Auflösungsversuche aus dieser Arbeit mit Daten aus der Literatur zusammengestellt und in Abbildung 6-8 graphisch dargestellt.

Tab. 6-8: Reaktionsordnungen n für protonengesteuerte Auflösung von Kaolinit bei $20-25^{\circ} \mathrm{C}$ (a: $n$ basiert auf 2 Datenpunkten).

\begin{tabular}{lccccl}
\hline \multicolumn{1}{c}{ Lösung } & Methode & $\begin{array}{c}\mathrm{pH}- \\
\text { Bereich }\end{array}$ & $\mathrm{n}$ & $\begin{array}{c}\text { Fehler (n) } \\
( \pm)\end{array}$ & Autor \\
\hline \hline $\mathrm{H}_{2} \mathrm{SO}_{4}$ & Durchfluß & $1-2$ & 0,42 & 0,10 & diese Arbeit \\
$\mathrm{HCl}$ & Durchfluß & $1-3,5$ & 0,35 & 0,06 & diese Arbeit \\
$\mathrm{H}_{2} \mathrm{SO}_{4}$ & Batch & $1-3$ & 0,41 & 0,05 & diese Arbeit \\
$\mathrm{H}_{2} \mathrm{SO}_{4}$ & Mixed flow & $3-6$ & 0,06 & $\mathrm{a}$ & Heydemann (1966) \\
$0,1 \mathrm{M} \mathrm{NaNO}_{3} / \mathrm{HNO}_{3}$ & Batch & $3-4$ & 0,38 & 0,04 & Wieland \& Stumm (1992) \\
$0,1 \mathrm{M} \mathrm{NaNO}_{3} / \mathrm{HNO}_{3}$ & Batch & $4-6,5$ & 0,09 & 0,01 & Wieland \& Stumm (1992) \\
$\mathrm{Puffer} \mathrm{HCl}_{\mathrm{HClO}_{4}}$ & Batch & $1-4$ & 0,12 & 0,05 & Carroll \& Walther (1990) \\
\hline
\end{tabular}

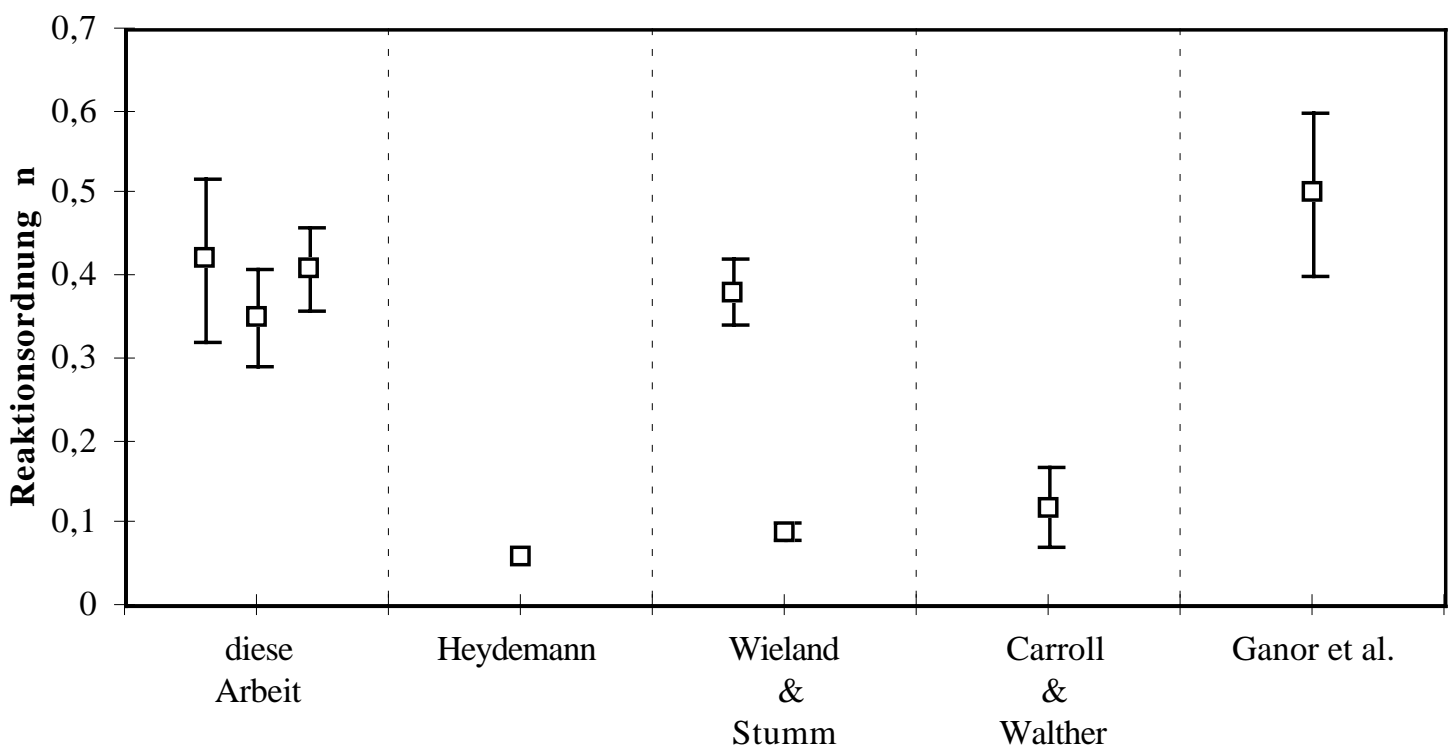

Abb. 6-8: Vergleich der ermittelten Reaktionsordnungen $n$ mit Daten aus der Literatur (vgl. Tab. 6-8). 
Zusammenfassend läßt sich über das Auflösungsverhalten von Kaolinit sagen:

bei protonenkontrollierten Prozessen können sich Kaolinite in $\mathrm{H}_{2} \mathrm{SO}_{4}$-saurer Lösung im pHBereich 1 - 3 kongruent auflösen (in der Lösung $\mathrm{R}_{\mathrm{Si}} \approx \mathrm{R}_{\mathrm{Al}}$ ), in $\mathrm{HNO}_{3}$ - oder $\mathrm{HCl}$-saurer Lösung gehen Kaolinite auch bei $\mathrm{pH} \leq 3$ nichtstöchiometrisch in Lösung (in der Lösung im allgemeinen $\mathrm{R}_{\mathrm{Si}}>\mathrm{R}_{\mathrm{Al}}$ ), mit steigendem $\mathrm{pH}$ nimmt die Lösungsgeschwindigkeit von $\mathrm{R}_{\mathrm{Si}}$ und $\mathrm{R}_{\mathrm{Al}} \mathrm{ab}$;

bei ligandengesteuerten Prozessen, also beim Einsatz bestimmter anorganischer oder organischer Säuren oder deren Salzen kann es auch im $\mathrm{pH}$-Bereich $\geq 3$ zu kongruenter Auflösung kommen, in $\mathrm{HClO}_{4}$-saurer Lösung beobachtet man dies noch bei pH 4 .

Es wurde auch beobachtet, daß die Auflösungsgeschwindigkeiten in ligandengesteuerten Systemen größer sind als in vergleichbaren protonengesteuerten Versuchen.

Vor allem die Al-Konzentration in den Lösungen wird nicht allein kontrolliert durch die Auflösungsgeschwindigkeit der Kaolinite, vielmehr spielen Adsorptions- und Komplexierungseffekte eine wichtige Rolle. Wieland \& Stumm (1992) deuteten bei der Interpretation ihrer Versuchsergebnisse auch noch daraufhin, daß man wohl selbst im stärker sauren $\mathrm{pH}$-Bereich mit der Ausfällung Al-reicher Präzipitate rechnen müßte. Im pH-Bereich $\geq$ 4,5 kann es aufgrund der Löslichkeitsdaten zur Ausfällung von amorphen Al-Hydroxiden und Gibbsit kommen (Dietzel 1998, Raupach 1960). Nach Nagy \& Sturchio (1997) soll die Ausfällung von Gibbsit bevorzugt auf der Tetraederschicht erfolgen. In $\mathrm{H}_{2} \mathrm{SO}_{4}$-saurer Lösung kann es im stärker sauren Bereich zur Bildung von Jurbanit oder Basaluminit kommen (Nordstrom 1982). In Abbildung 6-9 sind entsprechende Stabilitätsdiagramme zusammengestellt. Die Al-Konzentrationen in den Batch-Experimenten scheinen noch zu niedrig zu sein, um die Löslichkeitsprodukte solcher Al-Hydroxy-Sulfate oder Al-Hydroxide überschritten zu haben. Geeignete Bedingungen könnten sich in Mikromilieus ausbilden, was durch die ständige Durchmischung der Suspension aber weitgehend ausgeschlossen werden kann. 
(a)

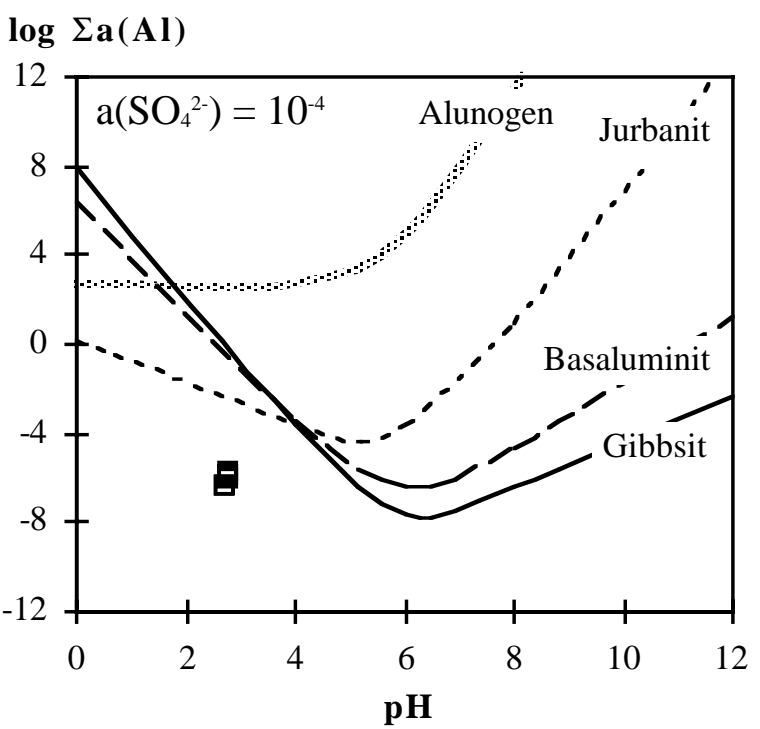

(b)

$\log \Sigma \mathbf{a}(\mathrm{Al})$

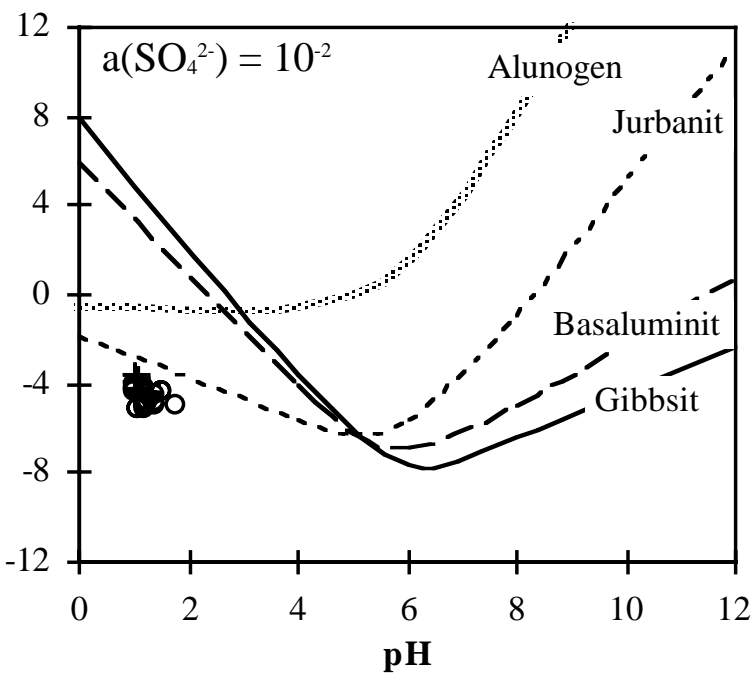

Abb. 6-9: Stabilitätskurven für Alunogen $\left(\mathrm{Al}_{2}\left(\mathrm{SO}_{4}\right)_{3} \bullet 17 \mathrm{H}_{2} \mathrm{O}\right)$, Jurbanit $\left(\mathrm{AlOHSO}_{4}\right)$, Basaluminit $\left(\mathrm{Al}_{4}(\mathrm{OH})_{10} \mathrm{SO}_{4}\right)$ und Gibbsit $\left(\mathrm{Al}(\mathrm{OH})_{3}\right)$ nach Nordstrom (1882) für eine $\mathrm{SO}_{4}{ }^{2-}$ Aktivität von $10^{-4}$ (a) bzw. $10^{-2}(b)$ mol/l. Die Datenpunkte repräsentieren die Versuche $3($ a) bzw. 1 und $7(b)$.

Welchen Einfluß derartige Mineralausfällungen z.B. von Gibbsit oder Jurbanit auf den Partikeloberflächen auf den Auflösungsprozeß haben, muß noch eingehender untersucht werden (Arias et al. 1995). 


\subsection{Montmorillonit}

Die Montmorillonite gehören zu den Dreischichtmineralen. Sie besitzen eine deutlich geringere negative Schichtladung als die Glimmer. Sie beruht vor allem auf Substitution von $\mathrm{Al}^{3+}$ durch $\mathrm{Mg}^{2+}$ in den Oktaederpositionen, Substitution in der Tetraederschicht ist nur gering. Der Ladungsausgleich erfolgt durch hydratisierte Kationen, die auf den Zwischenschichtpositionen sitzen und gegen andere Kationen ausgetauscht werden können. Diese Eigenschaft des Kationenaustausches verbunden mit der großen Oberfläche (> $600 \mathrm{~m}^{2} / \mathrm{gTon}$ ) verleiht den Montmorilloniten eine sehr hohe Kationenaustauschkapazität. Sie liegt bei 70 - 120 meq/100 g Montmorillonit im Vergleich zu 1 - 10 meq/100 g Kaolinit (Jasmund \& Lagaly 1993). Das bedeutet, die $\mathrm{pH}$ unabhängige Oberflächenladung ist relativ groß.

Bei der hier verwendeten Montmorillonitprobe handelt es sich um sehr feinkörniges Material $(<0,63 \mu \mathrm{m} \emptyset)$ mit überwiegend $\mathrm{Na}^{+}$-Ionen auf den Zwischenschichtplätzen. Die FEMAufnahme (Abb. 6-10) zeigt gelartige Strukturen, die keine Beschreibung der Oberflächenmorphologie zulassen. Neben den gelartigen Strukturen sind in der FEM-Aufnahme noch kleine Partikel zu erkennen (Abb. 6-10, Pfeile).

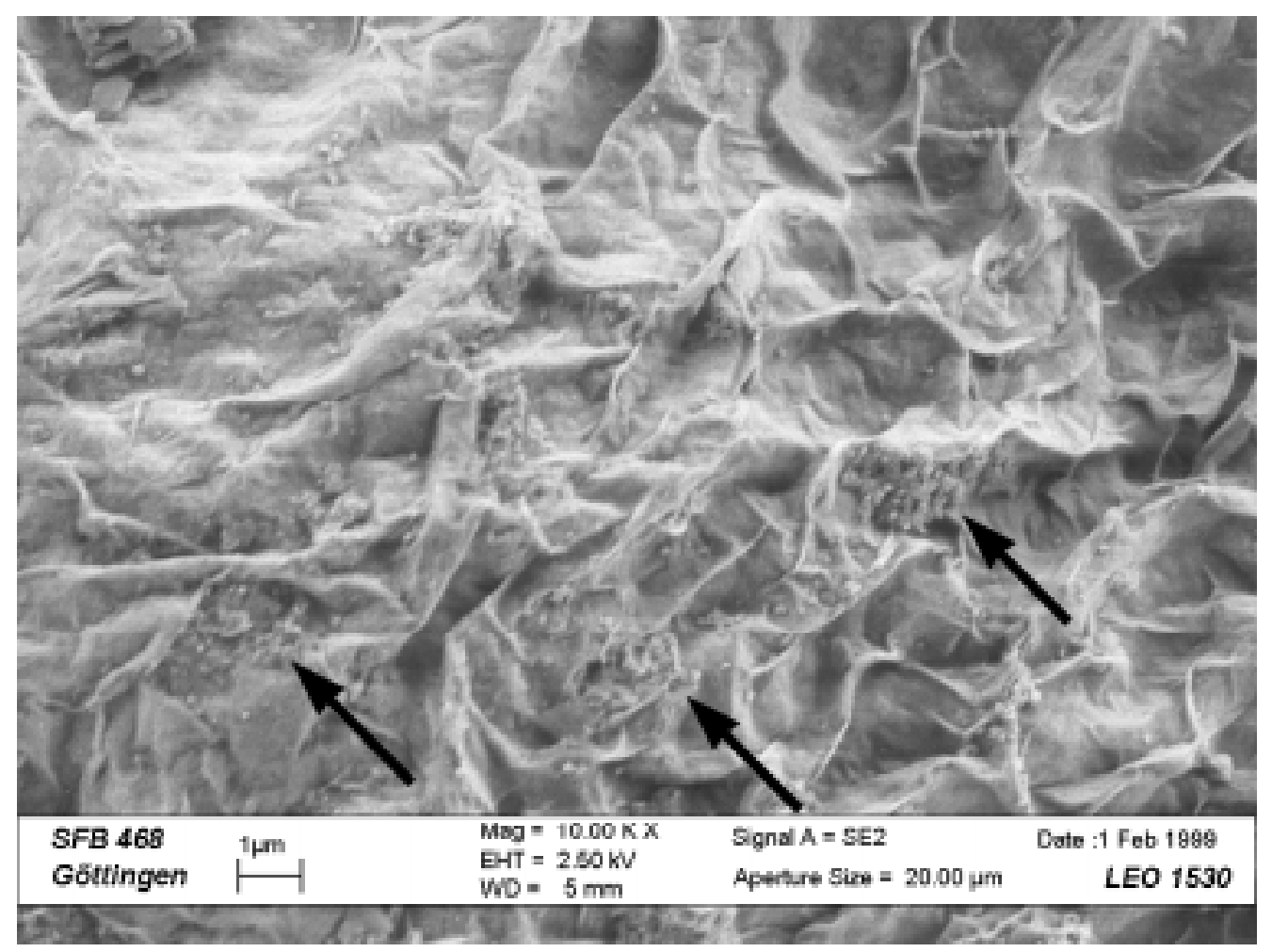

Abb. 6-10: FEM-Aufnahme des unbehandelten Montmorillonites. Bereiche mit einer Häufung sehr kleiner Partikel sind mit Pfeilen gekennzeichnet. 
In Abb. 6-11 ist die zeitliche Entwicklung eines Durchfluß-Experimentes mit dem Montmorillonit in $\mathrm{H}_{2} \mathrm{SO}_{4}$-saurer Lösung bei pH 1 dargestellt. Bereits nach ca. 20 Tagen hat sich quasi ein Gleichgewicht eingestellt. Das im Bereich von 60 - 80 Tagen in der Lösung gemessene mittlere Si/Al-Molverhältnis ist mit einem Wert von 2,45 identisch mit dem des Festkörpers $(\approx 2,44)$. Fe und $\mathrm{Mg}$ nehmen zunächst deutlich ab, was eventuell durch die Auflösung sehr feiner Partikel bedingt ist. Sie sind aber bis zum Ende des Experimentes in den Output-Lösungen nachweisbar. Mit Werten von $\mathrm{Si} / \mathrm{Fe} \approx 18,8$ und $\mathrm{Si} / \mathrm{Mg} \approx 16,0$ in der Lösung liegen im Bereich des „steady state” etwas geringere Werte vor als im Festkörper (Festkörper: $\mathrm{Si} / \mathrm{Fe} \approx 21,73 ; \mathrm{Si} / \mathrm{Mg} \approx 17,00)$. Man wird trotzdem von stöchiometrischer Auflösung des Montmorillonites sprechen können.

Das Na aus der Zwischenschicht wird bereits innerhalb der ersten 20 Tage vollständig gegen $\mathrm{H}^{+}$-Ionen ausgetauscht und ist dann in den Output-Lösungen nicht mehr nachweisbar.

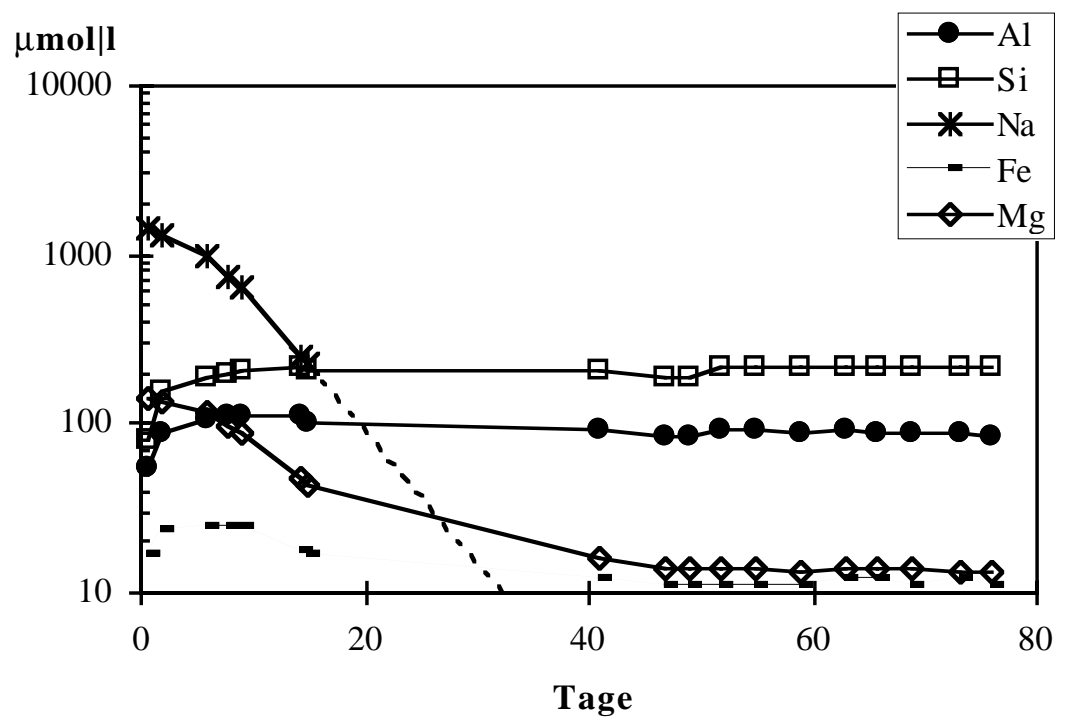

Abb. 6-11: Auflösung von Montmorillonit im Durchfluß-Experiment mit $\mathrm{H}_{2} \mathrm{SO}_{4}$ bei pH 1 (vgl. Versuch $\operatorname{VIII}(1))$.

Bei pH 2 und pH 3 sind ähnliche Versuchsverläufe zu beobachten mit kongruenter Auflösung im ,steady state”. Natürlich nimmt mit steigendem pH-Wert die Auflösungsgeschwindigkeit ab (Tab. 6-9). Man kann also im pH-Bereich 1 - 3 in $\mathrm{H}_{2} \mathrm{SO}_{4}$-sauren Lösungen von stöchiometrischer Auflösung des Montmorillonites sprechen. Auch bei Verwendung von $\mathrm{C}_{6} \mathrm{H}_{8} \mathrm{O}_{7}$ ist der Versuchsablauf vergleichbar, also kongruente Auflösung im „steady state” bei $\mathrm{pH} 2,5$. 
Tab. 6-9: Auflösungsraten $R$ und mittlere Mol-Verhältnisse im „steady state” für DurchflußExperimente mit Montmorillonit in $\mathrm{H}_{2} \mathrm{SO}_{4}$-saurer Lösung bei pH 1, 2 und 3 (vgl. Versuche $\operatorname{VIII}(1)$, VIII(2) und $\operatorname{VIII}(3))$.

\begin{tabular}{|c|c|c|c|c|c|c|c|}
\hline \multirow[b]{2}{*}{$\mathrm{H}_{2} \mathrm{SO}_{4}$} & \multicolumn{4}{|c|}{$\begin{array}{c}50 \mathrm{mg} \text { Montmorillonit / } 250 \mathrm{ml} \\
\mathrm{R} \text { in }[\mathrm{mol} /(\mathrm{g} \bullet \mathrm{h})]\end{array}$} & \multicolumn{3}{|c|}{$\begin{array}{c}\text { mittlere Mol-Verhältnisse im } \\
\text { Bereich des „steady state” }\end{array}$} \\
\hline & $\mathrm{R}_{\mathrm{Si}}$ & $\mathrm{R}_{\mathrm{Al}}$ & $\mathrm{R}_{\mathrm{Fe}}$ & $\mathrm{R}_{\mathrm{Mg}}$ & $\mathrm{Si} / \mathrm{Al}$ & $\mathrm{Si} / \mathrm{Fe}$ & $\mathrm{Si} / \mathrm{Mg}$ \\
\hline pH 1 & $1,0 \cdot 10^{-6}$ & $4,1 \cdot 10^{-7}$ & $5,3 \cdot 10^{-8}$ & $6,3 \cdot 10^{-8}$ & 2,45 & 18,8 & 16,0 \\
\hline pH 2 & $3,5 \cdot 10^{-7}$ & $1,5 \cdot 10^{-7}$ & $1,6 \bullet 10^{-8}$ & $1,8 \cdot 10^{-8}$ & 2,35 & 22,5 & 19,0 \\
\hline pH 3 & $1,3 \cdot 10^{-7}$ & $5,1 \cdot 10^{-8}$ & $7,4 \cdot 10^{-9}$ & $1,4 \cdot 10^{-8}$ & 2,53 & 17,5 & 9,4 \\
\hline
\end{tabular}

Vergleicht man die aus den Durchfluß-Experimenten ermittelten Informationen, also kongruente Auflösung des Montmorillonites im pH-Bereich 1 - 3, mit den Ergebnissen der BatchVersuche, so ergeben sich deutliche Unterschiede. Die Reaktionsabläufe der Auflösung von Montmorillonit in $\mathrm{H}_{2} \mathrm{SO}_{4}$-saurer Lösung bei $\mathrm{pH} 1$ und $\mathrm{pH} 2$ sowie in $\mathrm{C}_{6} \mathrm{H}_{8} \mathrm{O}_{7}$-saurer Lösung bei pH 2,5 sind in Abbildung 6-12 nebeneinander dargestellt.

(a)

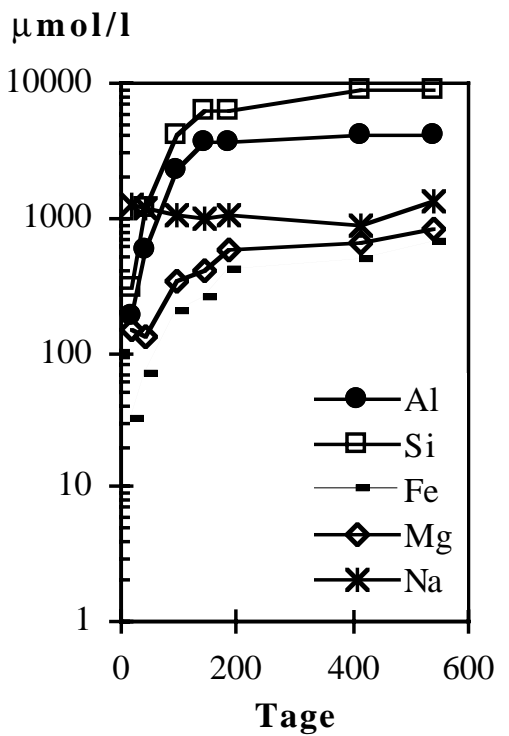

(b)

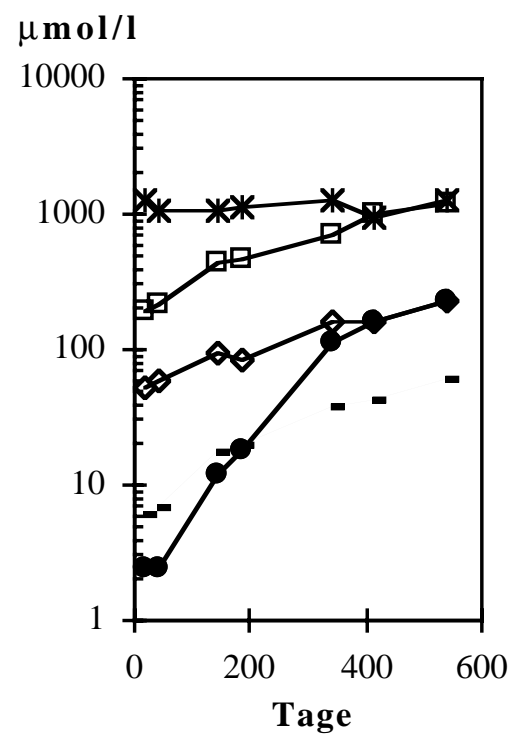

(c)

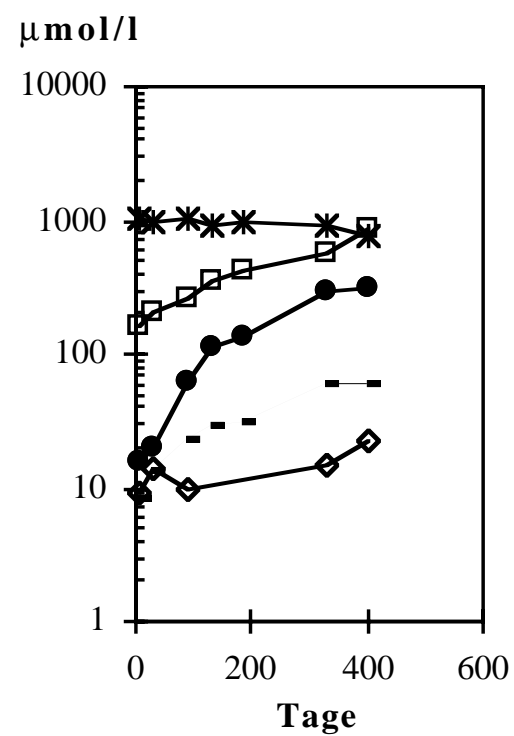

Abb. 6-12: Reaktionsabläufe von Batch-Versuchen mit Montmorillonit in $\mathrm{H}_{2} \mathrm{SO}_{4}$ ( und 2 (b)) sowie $\mathrm{C}_{6} \mathrm{H}_{8} \mathrm{O}_{7}$-sauren (c) Lösungen (vgl. Versuche 16, 17 und 26). 
Während bei den Durchfluß-Experimenten das $\mathrm{Na}$ bereits nach kurzer Versuchsdauer weitgehend aus dem System entfernt worden war, beobachtet man hier gleich zu Beginn der Versuche hohe Na-Konzentrationen in der Lösung. Der Austausch der $\mathrm{Na}^{+}$-Ionen aus dem Zwischenschichtraum gegen $\mathrm{H}^{+}$-Ionen geschieht sehr rasch.

Bei pH 1 erfolgt die Auflösung scheinbar kongruent (Abb. 6-12a), was sich aus den zu bestimmten Zeiten in der Lösung ermittelten Molverhältnissen $\mathrm{Si} / \mathrm{Al}$ sowie $\mathrm{Si} / \mathrm{Mg}$ und $\mathrm{Si} / \mathrm{Fe}$ ergibt (Tab. 6-10). Bemerkenswert ist, daß der Kurvenverlauf nach einer Versuchsdauer von $\approx$ 150 Tagen stark abflacht. Die Si- und Al-Kurven verlaufen dann fast parallel zur Abzisse. Der Auflösungsprozeß ist also stark verlangsamt (Tab. 6-10), die Elementkonzentrationen in der Lösung scheinen sich dem Sättigungswert in bezug auf Montmorillonit zu nähern (Furrer et al. 1993).

Tab. 6-10: Molverhältnisse und Auflösungsraten $R$ für ein Batch-Experiment mit Montmorillonit in $\mathrm{H}_{2} \mathrm{SO}_{4}$-saurer Lösung bei pH 1 (vgl. Versuch 16).

\begin{tabular}{cccccc}
\hline & \multicolumn{3}{c}{ Molverhältnisse } & \multicolumn{2}{c}{$\mathrm{R}$ in $[\mathrm{mol} /(\mathrm{g} \bullet \mathrm{h})]$} \\
Tage & $\mathrm{Si} / \mathrm{Al}$ & $\mathrm{Si} / \mathrm{Fe}$ & $\mathrm{Si} / \mathrm{Mg}$ & $\mathrm{R}_{\mathrm{Si}}$ & $\mathrm{R}_{\mathrm{Al}}$ \\
\hline \hline Montmorillonit & 2,4 & 22 & 17 & & \\
\hline bis 142 & 1,7 & 24 & 15 & $1,3 \bullet 10^{-6}$ & $7,9 \bullet 10^{-7}$ \\
$142-416$ & 2,1 & 18 & 14 & $2,7 \bullet 10^{-7}$ & $5,0 \bullet 10^{-8}$ \\
$416-539$ & 2,1 & 14 & 10 & $4,0 \bullet 10^{-8}$ & $1,3 \cdot 10^{-9}$ \\
\hline
\end{tabular}

Phasenneubildung als konzentrationskontrollierender Prozeß ist weitgehend auszuschließen. Aufgrund der Löslichkeitsdaten von Jurbanit (Abb. 6-9) wäre die Ausfällung dieser AlHydroxy-Sulfate möglich (Ball et al. 1982). Dies würde aber nur zu einer Reduzierung der AlKonzentration in der Lösung führen, die Si-, Fe- und Mg-Konzentrationen müßten weiter ansteigen.

Bei pH 2 deutet der Kurvenverlauf der Si- und Al-Konzentrationen auf einen anderen Reaktionsverlauf hin (Abb. 6-12b). Die Si-Konzentration steigt stetig an, während die AlKonzentration über den Versuchszeitraum zwar auch ansteigt, aber deutlich langsamer. Besonders ausgeprägt ist dies in den ersten 200 Tagen mit einem Si/Al-Molverhältnis in der Lösung > 20. Erst nach ca. 300 Tagen steigt die Al-Konzentration deutlicher an und es stellt sich nach 540 Tagen ein Si/Al-Molverhältnis von $\approx 5$ ein. Ein ähnlicher Trend war auch bei Untersuchungen mit anderen Montmorilloniten bei $\mathrm{pH} 3$ beobachtet und dort mit Adsorptionseffekten erklärt worden (Heydemann 1966). 
Nimmt man in Anlehnung an den Versuch bei pH 1 auch bei pH 2 kongruente Auflösung des Montmorillonites an, so kann man für die einzelnen Zeitabschnitte berechnen, wie groß der Anteil der adsorbierten $\mathrm{Al}^{3+}$-Ionen in bezug auf die insgesamt in Lösung gegangene Al-Menge ist (Tab. 6-11). Statt von Adsorption sollte man in diesem Zusammenhang vielleicht besser von einem Ionenaustausch von $\mathrm{H}^{+}$- gegen $\mathrm{Al}^{3+}$-Ionen sprechen.

Tab. 6-11: Auflösungsvorgang von Montmorillonit in $\mathrm{H}_{2} \mathrm{SO}_{4}$-saurer Lösung bei pH 2 im Batch-Experiment (vgl. Versuch 17).

\begin{tabular}{cccccccc}
\hline & & {$[\mu \mathrm{mol} / \mathrm{l}]$} & & \multicolumn{4}{c}{ Konzentrationen in $[\mu \mathrm{mol}]$ bezogen auf } \\
Tage & {$[\mathrm{Si}]_{\text {gel }}$} & {$[\mathrm{Al}]_{\text {theor }}$} & {$[\mathrm{Al}]_{\text {gel }}$} & {$[\mathrm{Si}]_{\mathrm{gel}}$} & {$[\mathrm{Al}]_{\text {theor }}$} & {$[\mathrm{Al}]_{\text {gel }}$} & {$[\mathrm{Al}]_{\text {ads }}$} \\
\hline \hline 19 & 188 & 75 & 2,4 & 18,8 & 7,5 & 0,2 & 7,3 \\
40 & 212 & 85 & 2,4 & 21,2 & 8,5 & 0,2 & 8,3 \\
142 & 435 & 174 & 12 & 43,5 & 17,4 & 1,2 & 16,2 \\
184 & 466 & 186 & 18 & 46,6 & 18,6 & 1,8 & 16,8 \\
341 & 693 & 277 & 110 & 69,3 & 27,7 & 11,0 & 16,7 \\
416 & 1025 & 410 & 158 & 102,5 & 41,0 & 15,8 & 25,2 \\
539 & 1218 & 487 & 224 & 121,8 & 48,7 & 22,4 & 26,3 \\
\hline gel: gelöst & \multicolumn{2}{c}{ theor: Al-Konzentration bei ungestörter kongruenter Auflösung } & ads: adsorbiert \\
\hline
\end{tabular}

Aus dem Ionenumtauschprozeß $\mathrm{Na}^{+}$gegen $\mathrm{H}^{+}$in der Anfangsphase des Versuches 17 kann man Rückschlüsse ziehen auf die Ionenaustauschkapazität der eingesetzten $150 \mathrm{mg}$ Montmorillonit, also der für den Austausch zur Verfügung stehenden permanenten Ladungspositionen. Es wurden $125 \mu \mathrm{mol} \mathrm{Na}{ }^{+}$von den $150 \mathrm{mg}$ Montmorillonit freigesetzt (Versuch 17, Tab. A-23). Das entspricht einer Ionenaustauschkapazität von $\approx 85 \mathrm{meq} / 100 \mathrm{~g}$ für diese Montmorillonitprobe. Sie liegt im Rahmen der bereits weiter oben angegebenen Literaturwerte für Smektite.

Es steht also bei Versuch 17 eine Ionenaustauschkapazität von ca. $125 \mu$ eq / $150 \mathrm{mg}$ Montmorillonit zur Verfügung. Nach 19 Tagen sind $\approx 22 \mu$ eq durch $\mathrm{Al}^{3+}$ besetzt, nach 539 Tagen $\approx 80 \mu$ eq. Bei diesem Ionenaustauschproze $\beta$ kommt es ständig zu neuer Gleichgewichtseinstellung zwischen gelösten und am Festkörper adsorbierten $\mathrm{Al}^{3+}$-Ionen. Mit steigender Al-Konzentration in der Lösung nimmt der Anteil adsorbierter Al ${ }^{3+}$-Ionen zu. Sind 
alle verfügbaren Austauschplätze mit $\mathrm{Al}^{3+}$ belegt, müßte der Auflösungsprozeß kongruent fortschreiten, bis der Sättigungszustand erreicht ist. Dieses Stadium ist bei dem Versuch 17 nicht erreicht worden. Die beschriebene Adsorption von $\mathrm{Al}^{3+}$-Ionen auf Kationenaustauschplätzen vor allem in den Zwischenschichten bildet vermutlich den ersten Schritt in Richtung pedogene Chloritbildung (Aluminiumchlorit), während Adsorption, z.B. an den Kanten, zur Verlangsamung des Auflösungsprozesses führen kann (Zysset \& Schindler 1996).

Der Versuchsablauf von Montmorillonit in $\mathrm{C}_{6} \mathrm{H}_{8} \mathrm{O}_{7}$-saurer Lösung (Abb. 6-12c) zeigt wieder in der Anfangsphase einen kompletten Austausch von $\mathrm{Na}^{+}$- gegen $\mathrm{H}^{+}$-Ionen. Der Auflösungsprozeß beginnt inkongruent (Tab. 6-12). Das Si/Al-Molverhältnis in der Lösung liegt zunächst bei 10, sinkt dann langsam und erreicht nach 405 Tagen einen Wert von 2,7 der mit dem Wert im Festkörper nahezu identisch ist (vgl. Tab. 5-1). Nach 405 Tagen scheint sich der Montmorillonit kongruent aufzulösen, wenn man nur das Si/Al-Molverhältnis in der Lösung betrachtet. Der Reaktionsablauf wird bei diesem Experiment stark beeinflußt durch die komplexierenden Eigenschaften von $\mathrm{C}_{6} \mathrm{H}_{8} \mathrm{O}_{7}$ auf die in der Lösung befindlichen $\mathrm{Al}^{3+}$-Ionen (Stone 1997). Sie stehen unter diesen Bedingungen kaum für den Ionenaustausch bzw. Adsorptionsprozesse zur Verfügung. Der Auflösungsprozeß ist also vor allem ligandenkontrolliert. Die Schwankungen in den Werten für die $\mathrm{Si} / \mathrm{Fe}$ - und die $\mathrm{Si} / \mathrm{Mg}$ Molverhältnisse, die im Verlauf des Auflöungsprozesses gemessen wurden, sind schwer zu interpretieren. Wahrscheinlich spielen auch hier Adsorptions- und Komplexierungsvorgänge eine Rolle (Scheffer \& Schachtschabel 1992, Stone 1997).

In $\mathrm{HNO}_{3}$-saurer Lösung laufen die Auflösungsprozesse im Batch-Experiment bei pH 1 und pH 2 ähnlich wie im $\mathrm{H}_{2} \mathrm{SO}_{4}$-sauren Milieu ab (Abb. 6-13).

Tab. 6-12: Auflösungsvorgang von Montmorillonit in $\mathrm{C}_{6} \mathrm{H}_{8} \mathrm{O}_{7}$-saurer Lösung im pH-Bereich 2,5 - 3,2 im Batch-Experimnent (vgl. Versuch 26).

\begin{tabular}{cccccc}
\hline \multicolumn{5}{c}{ Konzentrationen in $[\mu \mathrm{mol}]$} & \multicolumn{4}{l}{ bezogen auf $150 \mathrm{mg}$ Montmorillonit / } & $100 \mathrm{ml}$ Lösung \\
\hline \hline Tage & $\mathrm{Si} / \mathrm{Al} l_{\mathrm{Lsg} .}$ & {$[\mathrm{Si}]_{\text {gel }}$} & {$[\mathrm{Al}]_{\text {theor }}$} & {$[\mathrm{Al}]_{\mathrm{gel}}$} & {$[\mathrm{Al}]_{\mathrm{ads}}$} \\
8 & 10 & 16,1 & 6,4 & 1,6 & 4,8 \\
90 & 4,2 & 26,4 & 10,6 & 6,3 & 4,3 \\
405 & 2,7 & 84,4 & 33,8 & 31,6 & 2,2 \\
\hline \multirow{2}{*}{ gel: gelöst } & \multicolumn{2}{c}{ theor: Al-Konzentration bei ungestörter kongruenter Auflösung } & ads: adsorbiert \\
\hline
\end{tabular}


(a)

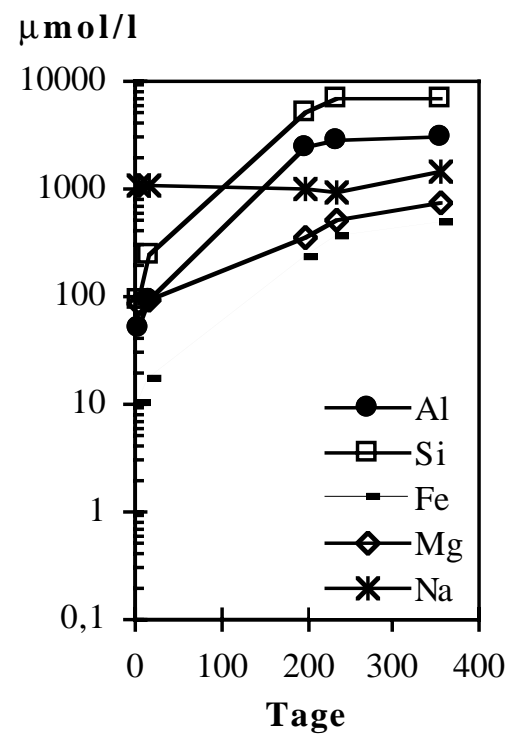

(b)

$\mu \mathbf{m o l} / \mathbf{l}$

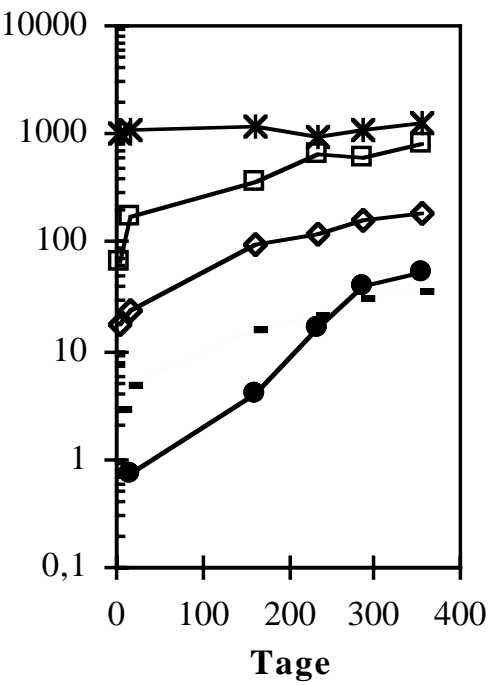

(c)

$\mu \mathbf{m o l} / \mathbf{l}$

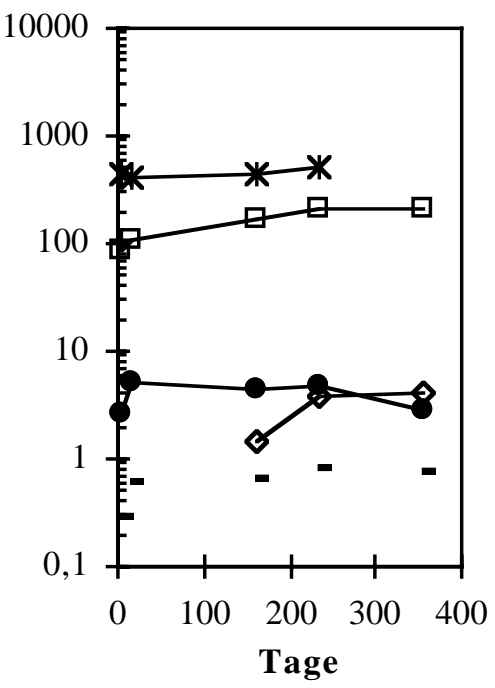

Abb. 6-13: Reaktionsabläufe von Batch-Versuchen mit Montmorillonit in $\mathrm{HNO}_{3}$-sauren Lösungen bei pH 1 (a), 2 (b) und im pH-Bereich 3 - 5 (c) (vgl. Versuche 37, 38 und 39).

Auch im $\mathrm{HNO}_{3}$-sauren Milieu beobachtet man kongruente Auflösung bei $\mathrm{pH} 1$ mit Erreichen des Sättigungswertes in bezug auf Montmorillonit nach $\approx 250$ Tagen. Die Si- und AlKonzentrationen steigen dann in der Lösung kaum noch an, die Auflösungsgeschwindigkeiten $\mathrm{R}_{\mathrm{Si}}$ und $\mathrm{R}_{\mathrm{Al}}$ tendieren gegen null. Bei $\mathrm{pH} 2$ ist der starke Einfluß von Adsorptions- bzw. Ionenaustauschprozessen auf die Al-Konzentrationen in den Lösungen zu beobachten. $\mathrm{Im} \mathrm{pH}-$ Bereich 3 - 5 (Abb. 6-13c) bemerkt man zunächst, daß wegen der geringeren $\mathrm{H}^{+}$ Ionenkonzentration in der Lösung nur ein teilweiser Austausch von $\mathrm{Na}^{+}$-Ionen gegen $\mathrm{H}^{+}$-Ionen stattfindet. Die Si-Konzentration steigt zu Beginn des Versuches rasch an, dann nur noch langsam. Die Lösungsgeschwindigkeit $\mathrm{R}_{\mathrm{Si}}$ nimmt also im Verlauf des Versuches ab (Abb. 613c, Tab. A-26). Die Konzentrationen von Al, Fe und Mg stagnieren dagegen von Beginn an auf niedrigem Niveau. Die entsprechenden Molverhältnisse bezogen auf Si liegen weit über den Werten im Festkörper. Neben dem Auflösungsprozeß müssen also andere Reaktionen parallel ablaufen. Die Mg-Konzentration in der Lösung wird sicher überwiegend durch Adsorptions/Ionenaustauschprozesse kontrolliert sein. Beim Al und Fe werden neben den Adsorptions/Ionenaustauschprozessen im $\mathrm{pH}$-Bereich $\geq 4$ auch Ausfällungsreaktionen der entsprechenden Hydroxide eine wesentliche Rolle spielen. 
Tab. 6-13: Auflösung von Montmorillonit in $\mathrm{HNO}_{3}$-saurer Lösung bei pH 2 (Versuch 38) und im pH-Bereich 3 - 5 (Versuch 39).

\section{Konzentrationen in $[\mu \mathrm{mol}]$ bezogen auf \\ $150 \mathrm{mg}$ Montmorillonit / $100 \mathrm{ml}$}

\section{Lösung}

Versuch Tage $\mathrm{pH} \quad \mathrm{Si} / \mathrm{Al} l_{\mathrm{Lsg} .} \quad[\mathrm{Si}]_{\text {gel }} \quad[\mathrm{Al}]_{\text {theor }} \quad[\mathrm{Al}]_{\text {gel }} \quad[\mathrm{Al}]_{\text {ads }}$ adsorbierter Anteil

\begin{tabular}{ccccccccc}
\multicolumn{1}{c}{38} & \multicolumn{1}{c}{0} & & \multicolumn{2}{c}{$\begin{array}{c}\text { des in Lösung } \\
\text { gegangenen Al [\% ] }\end{array}$} \\
& 2 & 2,0 & $>100$ & 6,8 & 2,7 & $<0,1$ & 2,7 & 100 \\
& 17 & 2,1 & $>100$ & 17,5 & 7,0 & $<0,1$ & 7,0 & 100 \\
& 160 & 2,1 & 95 & 37,2 & 14,9 & 0,4 & 14,5 & 97 \\
& 235 & 2,1 & 40 & 63,4 & 25,4 & 1,6 & 23,8 & 94 \\
& 288 & 2,0 & 16 & 62,4 & 25,0 & 3,9 & 21,1 & 84 \\
& 357 & 2,0 & 15 & 79,9 & 32,0 & 5,2 & 26,8 & 84 \\
& 2 & 3,6 & 33 & 8,7 & 3,5 & 0,3 & 3,2 & 91 \\
& 17 & 4,3 & 21 & 10,7 & 4,3 & 0,5 & 3,8 & 88 \\
& 160 & 3,1 & 37 & 16,3 & 6,5 & 0,4 & 6,1 & 94 \\
& 235 & 5,0 & 43 & 21,3 & 8,5 & 0,5 & 8,0 & 94 \\
357 & 4,5 & 75 & 21,8 & 8,7 & 0,3 & 8,4 & 97 \\
\hline
\end{tabular}

gel: gelöst theor: Al-Konzentration bei ungestörter kongruenter Auflösung ads: adsorbiert Lsg.: Lösung

Tab. 6-14: Zusammenstellung von $R_{S i}$-Daten für die Auflösung von Montmorillonit in Batchund Durchfluß-Experimenten im $p H$-Bereich 1 - 3 für verschiedene Säuren.

\begin{tabular}{cccccc}
\hline Typ & Versuch & $\mathrm{pH}$ & Säure & Zeitraum & $\mathrm{R}_{\mathrm{Si}}[\mathrm{mol} /(\mathrm{g} \bullet \mathrm{h})]$ \\
\hline \hline Batch & 16 & 1 & $\mathrm{H}_{2} \mathrm{SO}_{4}$ & $20-142$ Tage & $1,3 \cdot 10^{-6}$ \\
& & & & $416-539$ Tage & $4,0 \bullet 10^{-8}$ \\
Durchfluß & VIII(1) & 1 & $\mathrm{H}_{2} \mathrm{SO}_{4}$ & steady state & $1,0 \bullet 10^{-6}$ \\
Batch & 37 & 1 & $\mathrm{HNO}_{3}$ & $17-198$ Tage & $7,3 \cdot 10^{-7}$ \\
& & & & $235-357$ Tage & $6,6 \cdot 10^{-8}$ \\
Batch & 17 & 2 & $\mathrm{H}_{2} \mathrm{SO}_{4}$ & $20-539$ Tage & $5,5 \cdot 10^{-8}$ \\
Durchfluß & VIII(2) & 2 & $\mathrm{H}_{2} \mathrm{SO}_{4}$ & steady state & $3,5 \cdot 10^{-7}$ \\
Batch & 38 & 2 & $\mathrm{HNO}_{3}$ & $17-357$ Tage & $5,2 \cdot 10^{-8}$ \\
Batch & 26 & 2,5 & $\mathrm{C}_{6} \mathrm{H}_{8} \mathrm{O}_{7}$ & $8-405$ Tage & $4,4 \cdot 10^{-8}$ \\
Durchfluß & XIV & 2,5 & $\mathrm{C}_{6} \mathrm{H}_{8} \mathrm{O}_{7}$ & steady state & $5,9 \cdot 10^{-8}$ \\
Batch & 30 & 3 & $\mathrm{H}_{2} \mathrm{SO}_{4}$ & $17-357$ Tage & $1,3 \cdot 10^{-8}$ \\
Durchfluß & VIII(3) & 3 & $\mathrm{H}_{2} \mathrm{SO}_{4}$ & steady state & $1,3 \cdot 10^{-7}$ \\
Batch & 39 & 3 & $\mathrm{HNO}_{3}$ & $17-235$ Tage & $1,3 \cdot 10^{-8}$ \\
\hline
\end{tabular}


Betrachtet man die Lösungsgeschwindigkeiten $\mathrm{R}_{\mathrm{Si}}$ bei den verschiedenen Experimenten, so beobachtet man auch hier, daß sie mit steigendem pH-Wert abnehmen (Tab. 6-14, Abb. 6-13). Bei den $\mathrm{R}_{\mathrm{Al}}$-Werten ist dies wegen der zum Teil mit der Auflösung simultan ablaufenden Adsorptionsvorgänge nicht so eindeutig nachweisbar. Es sei hier nochmals darauf hingewiesen, daß sich bei den pH-1-Batch-Versuchen die Lösungsgeschwindigkeiten mit der Zeit erniedrigen, d.h. eine Annäherung an den Sättigungswert in bezug auf Montmorillonit erfolgt.

Zusammenfassend läßt sich über das Auflösungsverhalten von Montmorillonit sagen:

- Wird mit stärker sauren Lösungen ohne Elektrolytzusatz gearbeitet, so beobachtet man in der Anfangsphase der Experimente den Austausch der im Zwischenschichtraum sitzenden Kationen gegen $\mathrm{H}^{+}$-Ionen. Bei den Versuchen mit Na-Montmorillonit kann aus der Menge der bei $\mathrm{pH} 1$ und $\mathrm{pH} 2$ ausgetauschten $\mathrm{Na}^{+}$-Ionen die Kationenaustauschkapazität ermittelt werden.

- Bei protonenkontrollierten Auflösungsversuchen:

- Im Durchfluß-Experiment geht Montmorillonit im pH-Bereich 1 - 3 kongruent in Lösung.

- In Batch-Experimenten geht der Montmorillonit bei pH 1 ebenfalls kongruent in Lösung. Im Versuchsverlauf kann es zur Verminderung der Auflösungsgeschwindigkeit kommen, wenn durch Akkumulation die Elementkonzentrationen in der Lösung so hoch geworden sind, daß es zur Annäherung an den Sättigungswert in bezug auf Montmorillonit gekommen ist.

Im pH-Bereich 2 - 3 ist die kongruente Auflösung des Montmorillonits gekoppelt mit Adsorptionsvorgängen bzw. Ionenaustauschprozessen. Das Si/Al-Molverhältnis in der Lösung liegt weit über dem Wert des Festkörpers. Die in Lösung gegangenen $\mathrm{Al}^{3+}{ }^{+}$Ionen werden hier abhängig vom $\mathrm{pH}-$ Wert und ihrer Konzentration in der Lösung gegen $\mathrm{H}^{+}$ Ionen der Zwischenschicht ausgetauscht und zum Teil auch adsorbiert. Die AlKonzentration in der Lösung wird also kontrolliert durch die Gleichgewichtseinstellung zwischen ausgetauschten/adsorbierten und in Lösung befindlichen $\mathrm{Al}^{3+}$-Ionen.

- Werden die Auflösungsversuche mit Elektrolytzusatz durchgeführt, so sind die Ionenaustausch- und Adsorptionsprozesse weitgehend eingeschränkt.

- Bei ligandenkontrollierter Auflösung wird die Adsorption/der Ionenaustausch durch die Komplexierungsreaktionen weitestgehend unterbunden.

- Die Lösungsgeschwindigkeiten nehmen im allgemeinen mit steigendem pH-Wert ab. 
Ein Vergleich der hier ermittelten Untersuchungsergebnisse mit Literaturdaten ist im allgemeinen schwierig. Huang \& Keller (1971) finden in ihren ebenfalls bei geringer Ionenstärke durchgeführten Untersuchungen an zwei Montmorilloniten sowohl unter Verwendung von Wasser als auch unter Verwendung verschiedener organischer Säuren nichtstöchiometrisches ( $\mathrm{Si}>\mathrm{Al}$ ) Auflösungsverhalten. Metz \& Ganor (1999) konnten in ihren Versuchen mit $\mathrm{HClO}_{4}$-haltigen Lösungen stöchiometrische Auflösung beobachten. Gleiches gilt auch für Furrer et al. (1993) und Zysset \& Schindler (1996), die mit Lösungen relativ hoher Ionenstärke (0,03 - $1 \mathrm{M} \mathrm{KCl})$ noch bei $\mathrm{pH}$-Werten von 3,5 kongruente Auflösung beobachten konnten. Vermutlich sind bei den Versuchen von Metz \& Ganor (1999) wieder die bereits beim Kaolinit angesprochenen komplexierenden Eigenschaften von $\mathrm{HClO}_{4}$ verantwortlich bzw. bei den Versuchen von Zysset \& Schindler (1996) die Belegung der Adsorptionsplätze durch Ionen des Elektrolytzusatzes in den Input-Lösungen.

(a)

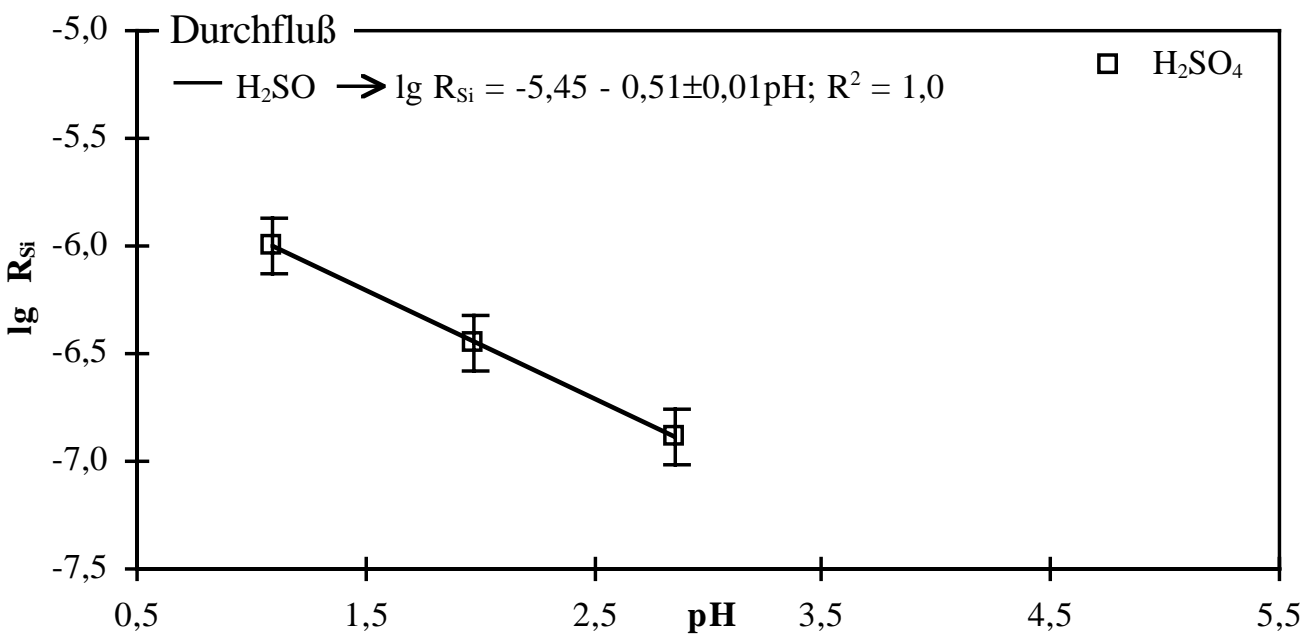

(b)

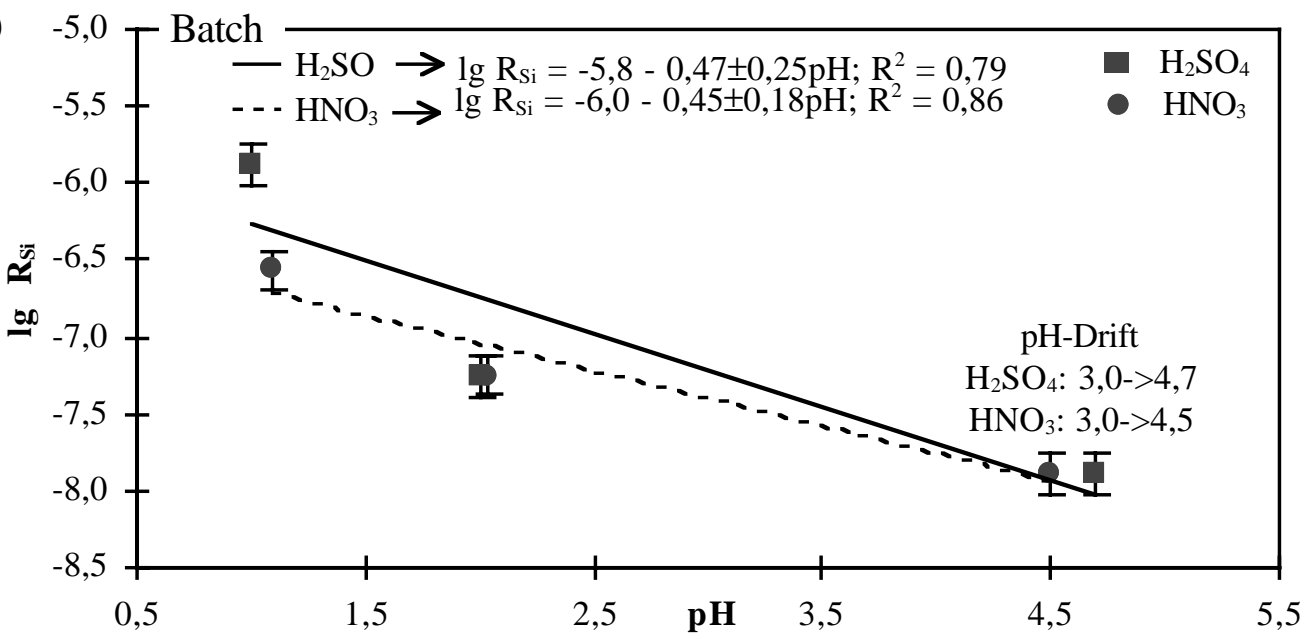

Abb. 6-14: Logarithmus der Auflösungsrate von Si $\left(R_{S i}\right)$ in Abhängigkeit vom pH-Wert für Batch- und Durchfluß-Experimente. Aus der Steigung der Geraden ergibt sich jeweils die Reaktionsordnung $n,(a)$ : Durchflu $\beta,(b)$ : Batch. 
In Abbildung 6-14 ist der Logarithmus der Auflösungsrate bezogen auf $\mathrm{Si}\left(\mathrm{R}_{\mathrm{Si}}\right)$ in Abhängigkeit vom pH-Wert aufgetragen. Aus der dargestellten Abhängigkeit läßt sich in Form der Steigung die Reaktionsordnung $n$ ablesen. Die durch das Erreichen der Sättigungskonzentration abnehmenden Auflösungsraten wurden hierbei nicht mit einbezogen.

In Abbildung 6-15 findet sich ein Vergleich der in dieser Arbeit ermittelten Reaktionsordnungen mit denen anderer Autoren. Es ergibt sich meistens eine recht gute Übereinstimmung der Werte. Eine Ausnahme bilden die von Zysset \&Schindler (1996) ermittelten höheren Werte (Tab. 615). In diesem Zusammenhang sollte erwähnt werden, daß sich wahrscheinlich Art und Dauer der Vorbehandlung des eingesetzten Tonmaterials auf die Auflösungsraten auswirken kann. Furrer et al. (1993) und Zysset \& Schindler (1996) benutzten für ihre Experimente beide den Na-Montmorillonit von Crook County, der vor den Versuchen in die K-Form überführt worden war. Beide Gruppen haben mit vergleichbaren Lösungen und $\mathrm{pH}-$ Werten gearbeitet, die unterschiedlichen Reaktionsordnungen sind vermutlich durch die jeweiligen Vorbehandlungen bedingt (Furrer et al. 1993).

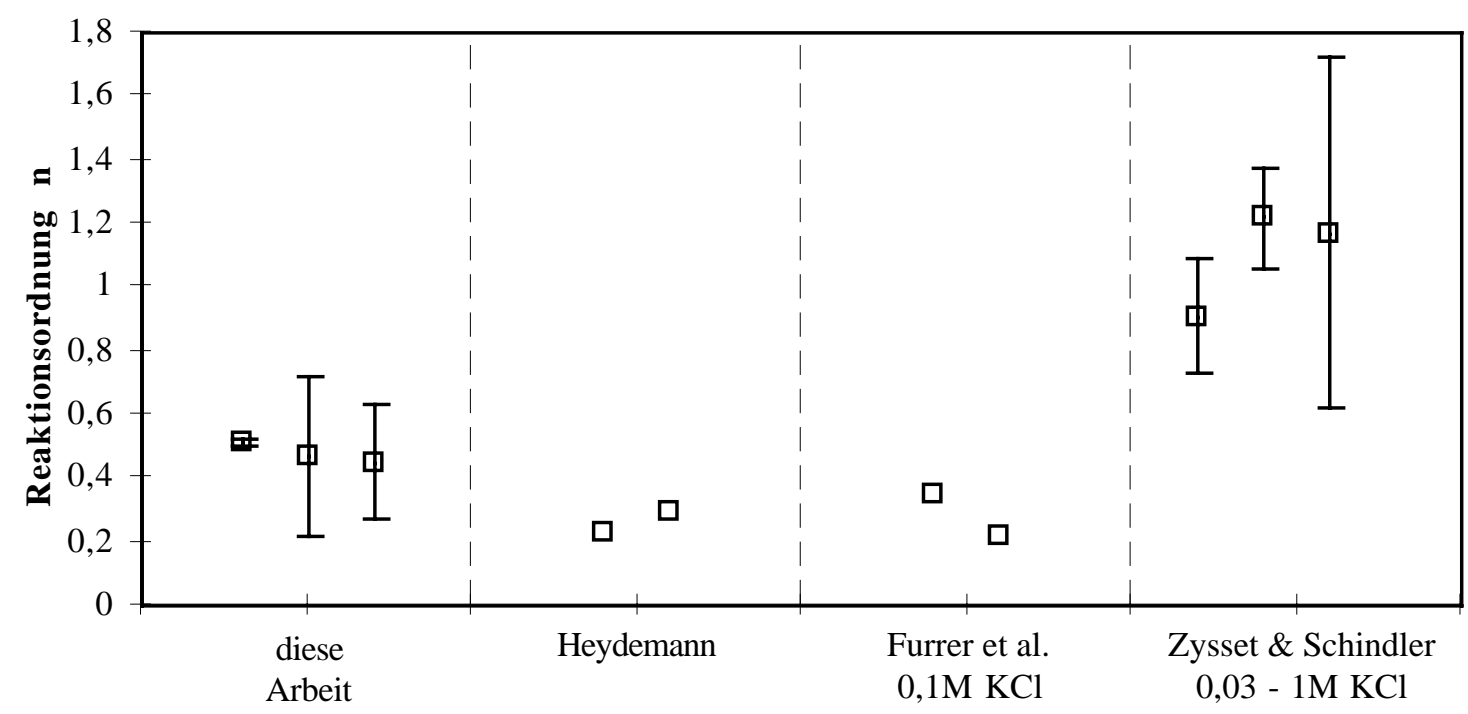

Abb. 6-15: Vergleich der ermittelten Reaktionsordnungen $n$ mit Daten aus der Literatur (vgl. auch Tab. 6-15). 
Tab. 6-15: Reaktionsordnungen $n$ für protonenkontrollierte Auflösung von Montmorillonit (a: $n$ basiert auf 2 Datenpunkten; k.A.: keine Angabe).

\begin{tabular}{lccccl}
\hline \multicolumn{1}{c}{ Lösung } & Methode & $\begin{array}{c}\text { pH- } \\
\text { Bereich }\end{array}$ & n & $\begin{array}{c}\text { Fehler (n) } \\
( \pm)\end{array}$ & Autor \\
\hline \hline $\mathrm{H}_{2} \mathrm{SO}_{4}$ & Durchfluß & $1-3$ & 0,51 & 0,01 & diese Arbeit \\
$\mathrm{H}_{2} \mathrm{SO}_{4}$ & Batch & $1-3$ & 0,47 & 0,25 & diese Arbeit \\
$\mathrm{HNO}_{3}$ & Batch & $1-3$ & 0,45 & 0,18 & diese Arbeit \\
$\mathrm{H}_{2} \mathrm{SO}_{4}$ & Mixed flow & $3-6$ & 0,23 & $\mathrm{a}$ & Heydemann (1966) \\
$\mathrm{H}_{2} \mathrm{SO}_{4}$ & Mixed flow & $3-6$ & 0,30 & a & Heydemann (1966) \\
$0,1 \mathrm{M} \mathrm{KCl} / \mathrm{HCl}$ & Batch & $1-4$ & 0,35 & k.A. & Furrer et al. (1993) \\
$0,1 \mathrm{M} \mathrm{KCl} / \mathrm{HCl}$ & Mixed-flow & $1-4$ & 0,22 & k.A. & Furrer et al. (1993) \\
$0,03 \mathrm{M} \mathrm{KCl} / \mathrm{HCl}$ & Batch & $1-4$ & 0,91 & 0,18 & Zysset \& Schindler (1996) \\
$0,10 \mathrm{M} \mathrm{KCl} / \mathrm{HCl}$ & Batch & $1-4$ & 1,22 & 0,16 & Zysset \& Schindler (1996) \\
$0,10 \mathrm{M} \mathrm{KCl} / \mathrm{HCl}$ & Batch & $1-4$ & 1,17 & 0,55 & Zysset \& Schindler (1996) \\
$1,0 \mathrm{M} \mathrm{KCl} / \mathrm{HCl}$ & Batch & $1-4$ & 0,85 & 0,04 & Zysset \& Schindler (1996) \\
\hline
\end{tabular}




\subsection{Serizit und Illit}

Als Vertreter der Glimmerminerale wurde ein sehr feinkörniger Muskovit ausgewählt, hier als Serizit bezeichnet. Die glimmerähnlichen Minerale werden durch zwei Illitproben vertreten, wobei die Illitprobe H-35 polymineralisch ist, also eher einer Bodenprobe entspricht, mit Illit als Hauptkomponente. Eine detaillierte Übersicht über die wichtigsten mineralogischen und chemischen Daten der Mineralproben ist in Tabelle 5-1 gegeben.

In Abbildung 6-16 ist der Auflösungsvorgang von Serizit (a), dem Illit OECD (b) und dem Illit H-35 (c) in $\mathrm{H}_{2} \mathrm{SO}_{4}$-saurer Lösung bei pH $1 \mathrm{im}$ Durchfluß-Experiment dargestellt. Für alle drei Mineralproben zeigen neben den $\mathrm{Si}$ - und Al-Konzentrationen auch die übrigen Gitterbausteine - Fe, Mg und K - einen prinzipiell ähnlichen Verlauf in der Lösung. Dieser ist gekennzeichnet durch abnehmende Elementkonzentrationen mit zunehmender Experimentdauer und der Einstellung des ,steady state” nach ca. 50 Tagen. Die im Vergleich zum Serizit deutlich höheren Elementkonzentrationen bei den Illit-Versuchen sind mit Sicherheit auf die unterschiedlichen Kornfraktionen zurückzuführen. Die beiden Illitproben sind im Gegensatz zum Serizit sehr feinkörnig (Abb. 6-17). Daraus resultiert eine größere Gesamtoberfläche und damit eine relativ große Kantenfläche. Beim Serizit zeigen die Mineralblättchen Durchmesser bis zu $50 \mu \mathrm{m}$ und sind sehr dünn. Der Anteil der Kantenflächen an der nicht sehr großen Gesamtoberfläche wird damit gering sein. Die in der Anfangsphase der Versuche in allen drei Proben zu beobachtenden höheren Konzentrationen resultieren vor allem aus der bevorzugten Auflösung feinster Partikel bzw. von Kornüberzügen.

Betrachtet man nun die Auflösungsprozesse im einzelnen, so muß man feststellen, daß sich der Serizit bei pH 1 annähernd kongruent auflöst. Die Werte für das Si/Al-Molverhältnis liegen im „steady state” bei 1,3 - 1,6, was etwa dem Wert im Serizit-Festkörper entspricht ( $\mathrm{Si} / \mathrm{Al} \approx 1,4$; vgl. Tab. 5-1 ). Auch das Si/Mg-Molverhältnis mit einem Wert von 9 -10 (im Festkörper $\approx 11$, vgl. Tab. 5-1) im ,steady state” deutet auf kongruente Auflösung hin. Der relativ hohe Anteil an Quarz in der Probe scheint kaum zur Si-Konzentration in der Lösung beizutragen. Das läßt sich dadurch erklären, daß unter gleichen Versuchsbedingungen im sauren $\mathrm{pH}$-Bereich die Lösungsraten für Quarz sehr niedrig sind (Heydemann 1966). Die Si/K-Molverhältnisse in der Lösung liegen im „steady state” mit 2,6 - 3,0 etwas zu niedrig verglichen mit dem Festkörperwert von 3,3 (vgl. Tab. 5-1).

Die Ursache für solche etwas zu niedrigen Si/K-Molverhältnisse in der Lösung hängt wohl mit dem Bautyp der Glimmer zusammen. Die Kalium-Kationen sitzen ja im Zwischenschichtraum. Sie sind über innersphärische Bindungen an die Schichtpakete gebunden. Anders als für Al und Si müssen für das Freisetzen aus dem Schichtpaket keine kovalenten Bindungen aufgebrochen werden. Die Freisetzung erfolgt vielmehr durch eine Art Ionenaustauschreaktion. Da das Aufbrechen solcher Bindungen wesentlich geringere Aktivierungsenergien benötigt, kann K also leichter aus dem Schichtverband freigesetzt werden (Huang \& Keller 1971). 


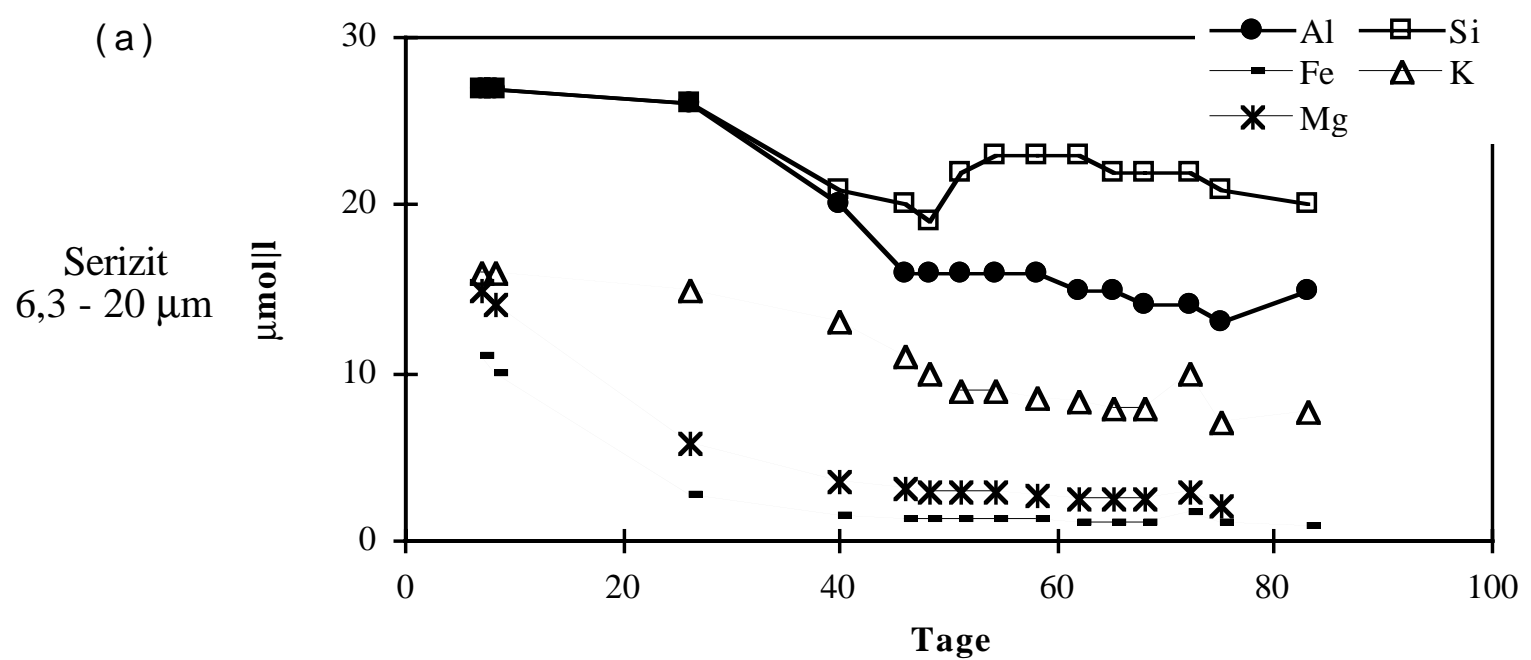

(b)

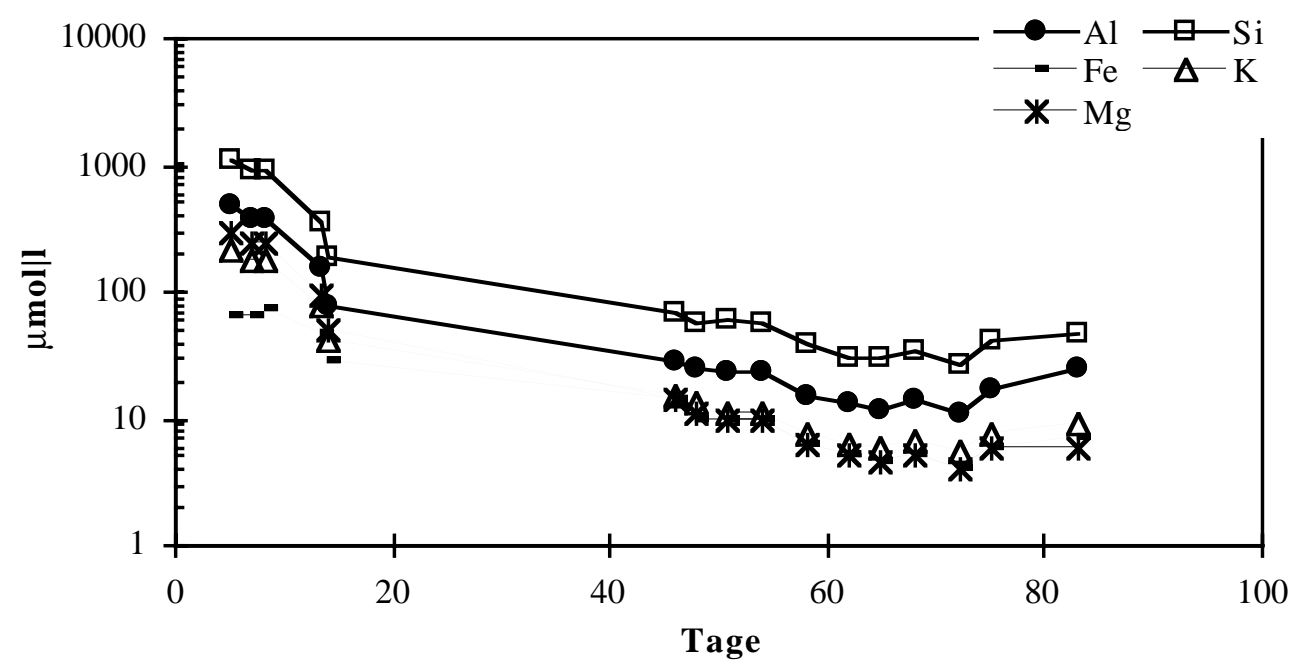

(c)

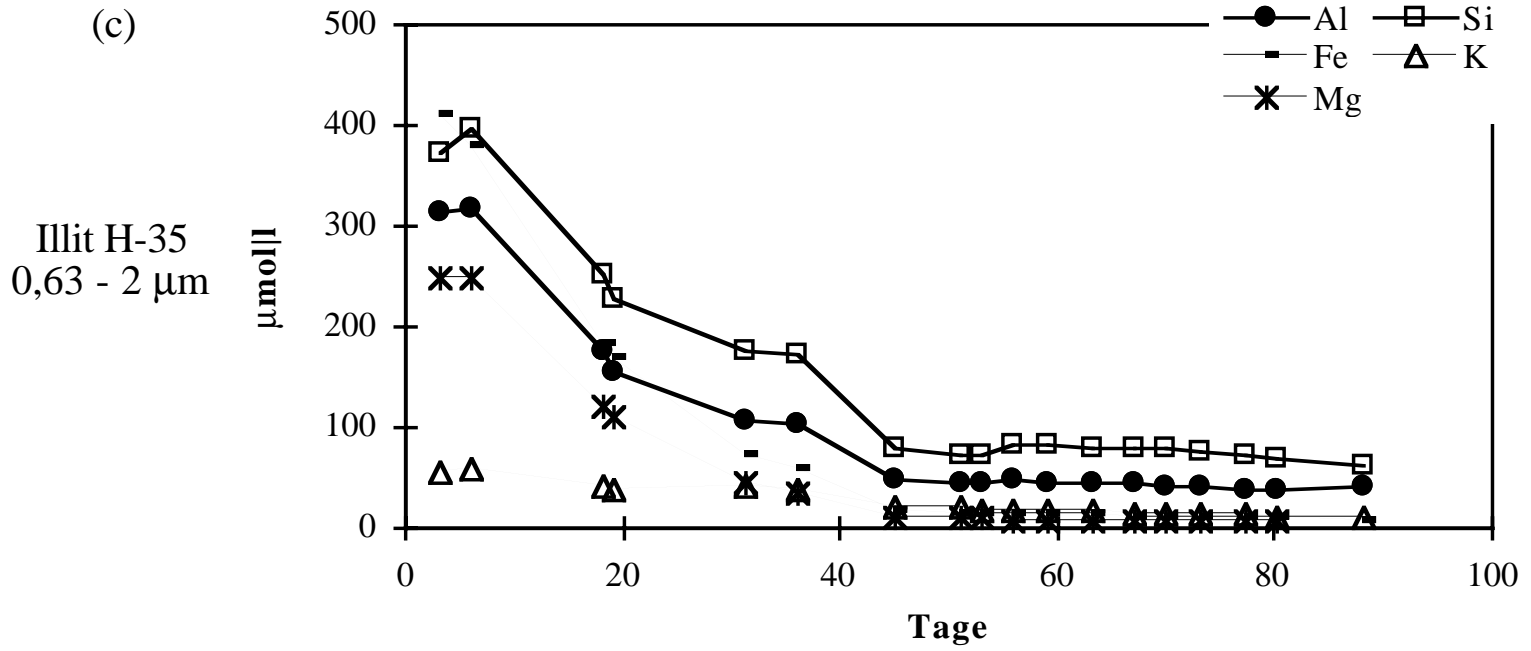

Abb. 6-16: Auflösung von Serizit (a), Illit OECD (b) und Illit H-35 (c) in DurchflußExperimenten in $\mathrm{H}_{2} \mathrm{SO}_{4}$-sauren Lösungen bei $\mathrm{pH}$ 1. Wegen der deutlich höheren Konzentrationen für den Illit OECD sind diese logarithmisch aufgetragen (vgl. Versuche (a): $\operatorname{IX}(1),(b): X I I(1),(c): \operatorname{VII}(1))$. 
Ergebnisse und Diskussion
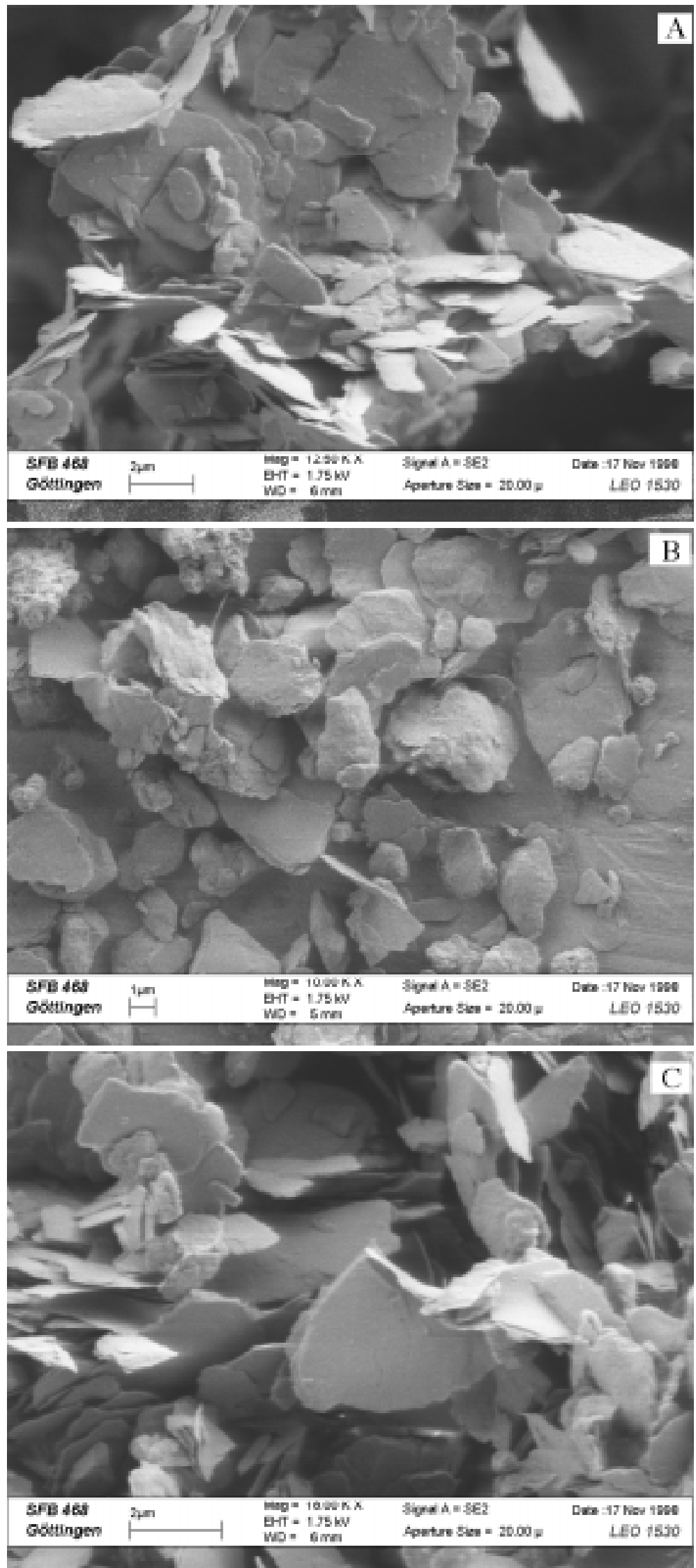

Abb. 6-17: FEM-Aufnahmen des unbehandelten Serizit (A), Illit OECD (B) und Illit H-35 (C). 
Nickel (1973) hatte dieses bevorzugte Herauslösen von K bei seinen Experimenten mit Muskovit ebenfalls beobachtet. Kalinowski \& Schweda (1996) beobachteten bei Untersuchungen zur Auflösung von Muskovit-Blättchen bei pH 1 kongruente Auflösung auch in bezug auf K, im pH-Bereich 2 - 4 jedoch auch bevorzugtes Herauslösen von K. Gleichzeitig von ihnen untersuchte Phlogopite und Biotite zeigten im gesamten $\mathrm{pH}$-Bereich bevorzugtes Herauslösen von $\mathrm{K}$.

Bei pH 2 liegen die Si/Al-Molverhältnisse in der Lösung bei > 2. Geht man auch in diesem Fall von kongruenter Auflösung aus, so lassen sich die Versuchsergebnisse nur interpretieren durch simultan mit der Auflösung ablaufende Reaktionen, wie z.B. der Adsorption von in Lösung gegangenen Al-Ionen. Die Konzentrationen der anderen Gitterbausteine Fe, Mg und $\mathrm{K}$ liegen wegen der abnehmenden Lösungsgeschwindigkeit mit steigendem $\mathrm{pH}$ schon an der unteren Grenze der jeweiligen Meßbereiche.

Die Veränderungen der Oberflächenmorphologie zeichnen sich beim Serizit durch die Bildung von Lösungsspuren und Ätzgruben aus (Abb. 6-18). Deutliche Rundungseffekte der Kanten sind hingegen nicht zu beobachten. Für den Serizit kann also ein nicht nur über die Mineralkanten erfolgender Auflösungsmechanismus beobachtet werden, der aber auch überwiegend zu kongruenter Auflösung führt. Die Bildung von Ätzgruben ist in der Regel an Defektstrukturen im Mineralgitter gebunden. Diese Strukturen weisen Überschußenergien auf und fungieren als Nukleationspunkte. Durch den Energieüberschuß an solchen Positionen reicht zum Herauslösen der Elemente aus der Gitterstruktur bereits eine geringere Aktivierungsenergie aus (Hochella \& Banfield 1995). 


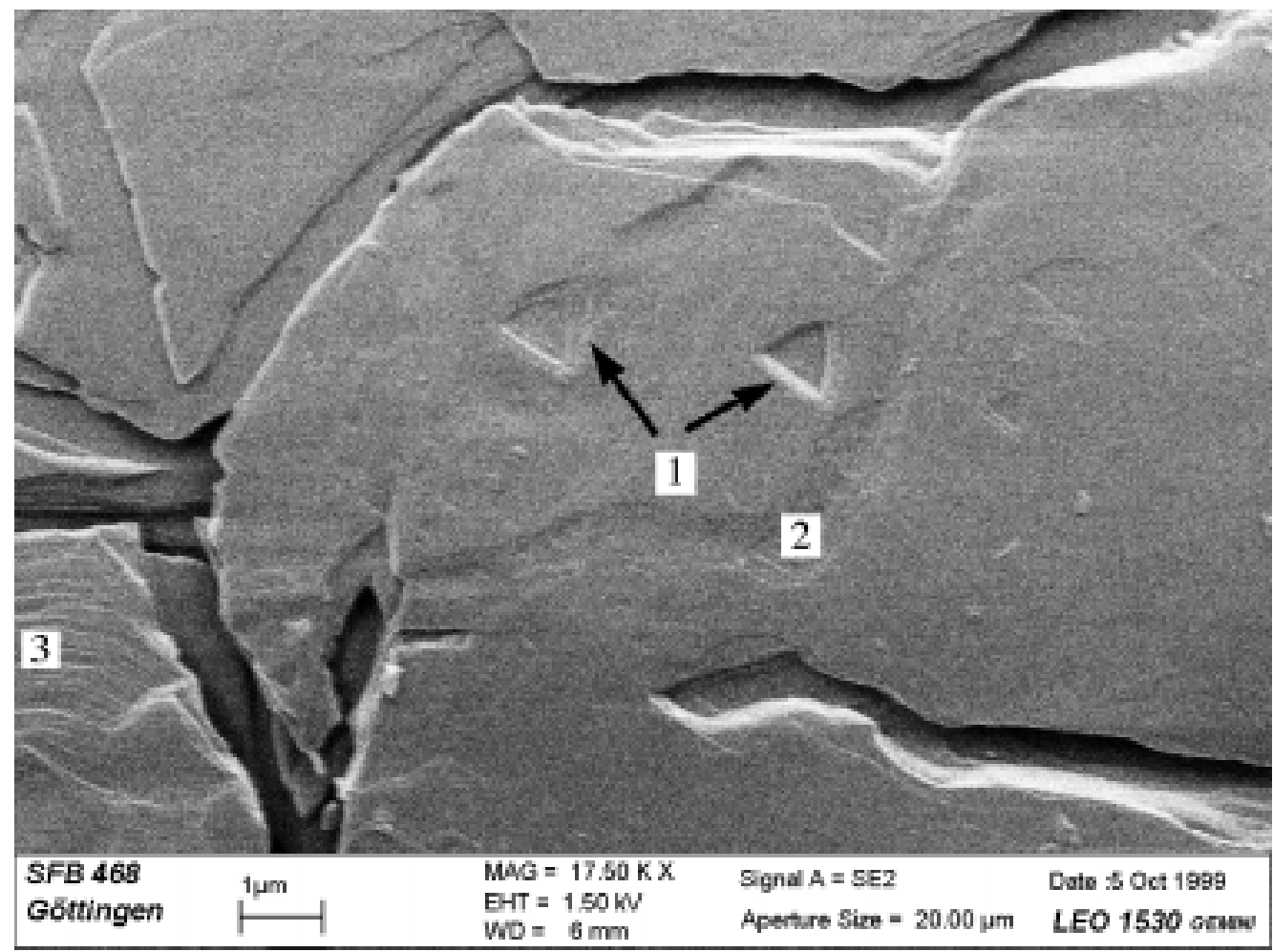

Abb. 6-18: FEM-Aufnahme von Serizit nach Behandlung mit $\mathrm{H}_{2} \mathrm{SO}_{4}$-saurer Lösung im Durchfluß-Experiment (1: Ätzgruben, 2: Lösungsspuren, 3: Stufen).

Auch beim Illit OECD erfolgt die Auflösung bei $\mathrm{pH} 1$ scheinbar kongruent, wenn man nur das Si/Al-Molverhältnis in der Lösung betrachtet (Tab. 6-16). Dieses liegt mit $\approx 2,5$ nahe dem Wert im Festkörper $(\approx 2,3$; vgl. Tab. 5-1). Bemerkenswert sind aber die zu niedrigen $\mathrm{Si} / \mathrm{Fe}-$ und $\mathrm{Si} / \mathrm{Mg}-$ Molverhältnisse in der Lösung. Feigenbaum \& Shainberg (1975) geben zwei Mechanismen an, die für das bevorzugte Herauslösen von $\mathrm{Mg}$ und $\mathrm{Fe}$ aus dem Illit verantwortlich sein können:

1) Die isomorphe Substitution in der Oktaederschicht eines Tonminerals schwächt die chemische Stabilität des Minerals. Die relativ großen $\mathrm{Mg}^{2+}$ - und $\mathrm{Fe}^{3+} / \mathrm{Fe}^{2+}$-Ionen passen nicht optimal in die Oktaederposition, es kommt zu Verzerrungen, die dann eine Schwächung der Struktur zur Folge haben.

2) Das Aufbrechen der Al-O Bindungen in der Al-O-Si Verknüpfung erfordert mehr Energie als das Aufbrechen der Mg-O (oder Fe-O) Bindungen in den $\mathrm{Mg}-\mathrm{O}-\mathrm{Si}$ oder $\mathrm{Mg}-\mathrm{O}-\mathrm{Al}$ Verknüpfungen. Die Mg-O (oder Fe-O) Bindungen werden also zuerst von Protonen aktiviert, womit bevorzugt $\mathrm{Mg}^{2+}$ - oder $\mathrm{Fe}^{3+} / \mathrm{Fe}^{2+}$-Ionen in die Lösung abgegeben werden. 
Die Tatsache, daß bestimmte oktaedrisch gebundene Ionen bevorzugt aus dem Illitgitter in die Lösung gelangen, ist ein deutlicher Hinweis, daß Auflösung des Illitgitters der dominierende Mechanismus sein muß. Diffusionsprozesse spielen keine Rolle. Auch das leicht bevorzugte Herauslösen von $\mathrm{K}$, das ja zu den etwas zu niedrig liegenden Si/K-Molverhältnissen in der Lösung führt (Tab. 6-16), ist auf diese Auflösungsmechanismen zurückzuführen.

Tab. 6-16: Element-Molverhältnisse aus der Auflösung von Illit OECD in $\mathrm{H}_{2} \mathrm{SO}_{4}$-saurer Lösung bei pH 1 im Durchfluß-Experiment (vgl. Versuch XII(1)).

\begin{tabular}{lcccc}
\hline & $\mathrm{Si} / \mathrm{Al}$ & $\mathrm{Si} / \mathrm{Fe}$ & $\mathrm{Si} / \mathrm{Mg}$ & $\mathrm{Si} / \mathrm{K}$ \\
\hline \hline Illit OECD & 2,3 & 10 & 10 & 5,7 \\
\hline am 51. Tag gelöst & 2,5 & 6,1 & 6,1 & 5,5 \\
am 65. -"- & 2,5 & 6,5 & 6,5 & 5,2 \\
am 75. -"- & 2,5 & 7,1 & 7,2 & 5,5 \\
\hline
\end{tabular}

Bei pH 2 scheint sich der Illit OECD auch kongruent aufzulösen, wenn man nur das Si/AlMolverhältnis in der Lösung betrachtet. Aufgrund der niedrigen Auflösungsgeschwindigkeit bei pH 2 liegen die Konzentrationen der anderen Kationen an der unteren Grenze des Meßbereiches. Es kann also nichts ausgesagt werden über ein möglicherweise auch unter diesen Bedingungen bevorzugtes Herauslösen von Mg- oder Fe-Ionen.

Bei der illitreichen Probe H-35 sind bei pH 1 hohe Fe-Konzentrationen in der Lösung zu Beginn des Versuchs zu registrieren (Abb. 6-16c). Sie stammen mit Sicherheit aus der Auflösung der Fe-Oxidhydrat-Überzüge (vgl. Tab. 5-1). Die zunächst $<2$ liegenden Si/AlMolverhältnisse in der Lösung deuten entweder auf die Auflösung Al-reicher Phasen in der Anfangsphase hin oder einem zunächst leicht bevorzugten Herauslösen von Al-Ionen aus der Illit-Komponente zusammen mit den anderen oktaedrisch gebundenen Ionen (Tab. 6-17). Im „steady state” nähern sich die Si/Al-, Si/Mg- und Si/K-Molverhältnisse in der Lösung denen in der Hauptkomponente Illit an. Man kann hier wohl kongruente Auflösung der Illitkomponente annehmen. 
Tab. 6-17: Element-Molverhältnisse aus der Auflösung der Illitprobe $\mathrm{H}$-35 in $\mathrm{H}_{2} \mathrm{SO}_{4}$-saurer Lösung bei pH 1 im Durchfluß-Experiment (vgl. Versuch VII(1)).

\begin{tabular}{|c|c|c|c|c|}
\hline & $\mathrm{Si} / \mathrm{Al}$ & $\mathrm{Si} / \mathrm{Fe}$ & $\mathrm{Si} / \mathrm{Mg}$ & $\mathrm{Si} / \mathrm{K}$ \\
\hline Illit & 2,1 & 18 & 11 & 5,7 \\
\hline Gesamtprobe & 2,4 & 13 & 18 & 9,4 \\
\hline am 18. Tag gelöst & 1,4 & 1,4 & 2,1 & 6,3 \\
\hline am 56. -"- & 1,8 & 5,9 & 9,9 & 4,9 \\
\hline am 70. - -"- & 1,8 & 6,6 & 11 & 5,3 \\
\hline am 80. -"- & 1,9 & 7,1 & 11 & 5,8 \\
\hline
\end{tabular}

Ähnlich sind die Versuchsergebnisse bei pH 2 und pH 3 zu interpretieren. Auch hier ist zu bemerken, daß wegen der Abnahme der Auflösungsgeschwindigkeiten die Kationen $\mathrm{Fe}, \mathrm{Mg}$ und $\mathrm{K}$ in zu geringen Konzentrationen vorliegen, um bei der Interpretation berücksichtigt werden zu können.

Bei den Batch-Versuchen beobachtet man ähnliche Reaktionsprozesse wie bei den DurchflußExperimenten. In Abbildung 6-19 sind die Versuchsabläufe der Auflösung von Serizit in $\mathrm{H}_{2} \mathrm{SO}_{4}$-saurer Lösung bei pH 1 und $\mathrm{pH} 2$ dargestellt.

(a)

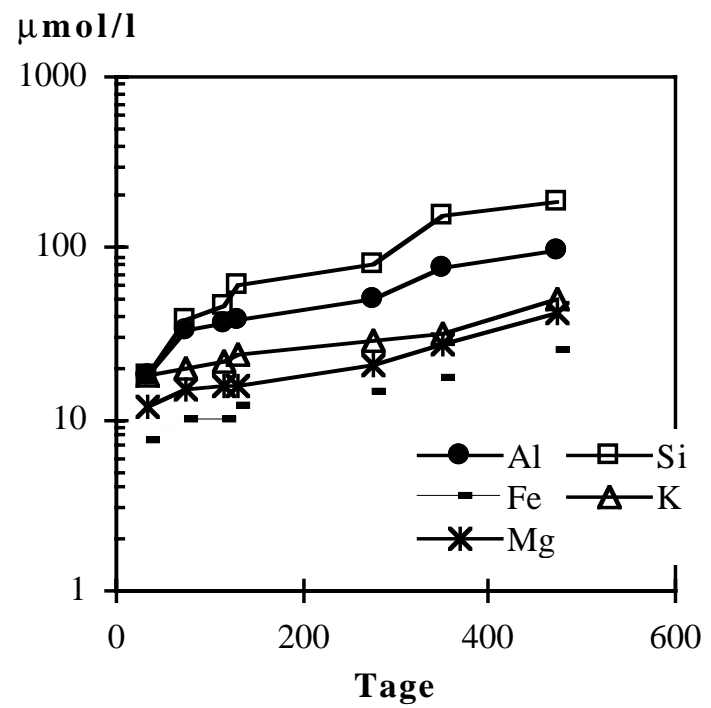

(b)

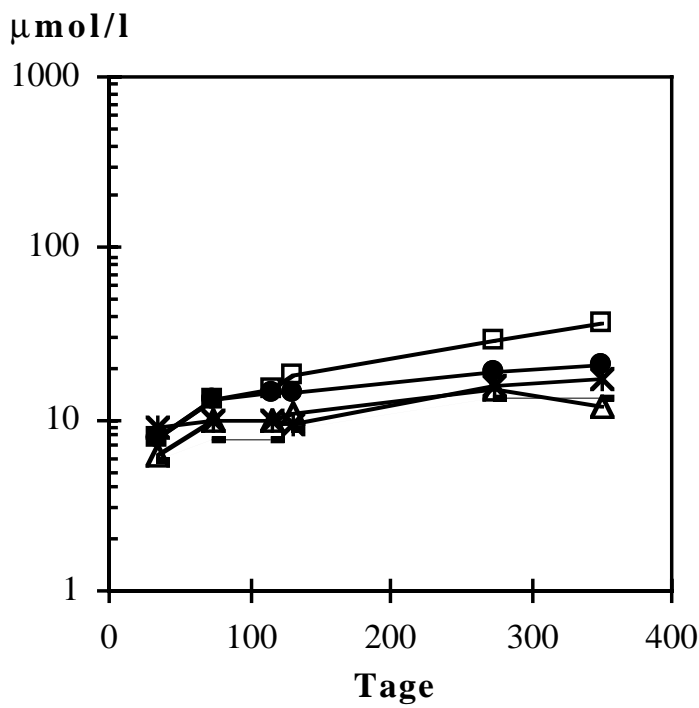

Abb. 6-19: Auflösung von Serizit in $\mathrm{H}_{2} \mathrm{SO}_{4}$-saurer Lösung bei pH 1 (a) und pH 2 (b) im Batch-Experiment (vgl. Versuche 21 und 24). 
In der Anfangsphase kommt es zunächst zur Auflösung sehr feinkörniger, möglicherweise auch amorpher Komponenten. Dann aber spiegeln die Kurvenverläufe die Auflösung des Serizits wider. Die Auflösung des Quarzes wird bei diesen Batch-Versuchen möglicherweise auch zur Si-Konzentration in der Lösung beitragen, da die Versuche über sehr lange Zeiträume durchgeführt wurden. Deutlich erkennbar ist die abnehmende Lösungsgeschwindigkeit mit steigendem $\mathrm{pH}-$ Wert.

In Abbildung 6-20 sind die Auflösungsvorgänge des Illits OECD in $\mathrm{H}_{2} \mathrm{SO}_{4}$-saurer Lösung bei pH 1 (a), pH 2 (b) und pH 3 (c) in den Batch-Versuchen zusammen dargestellt.

(a)

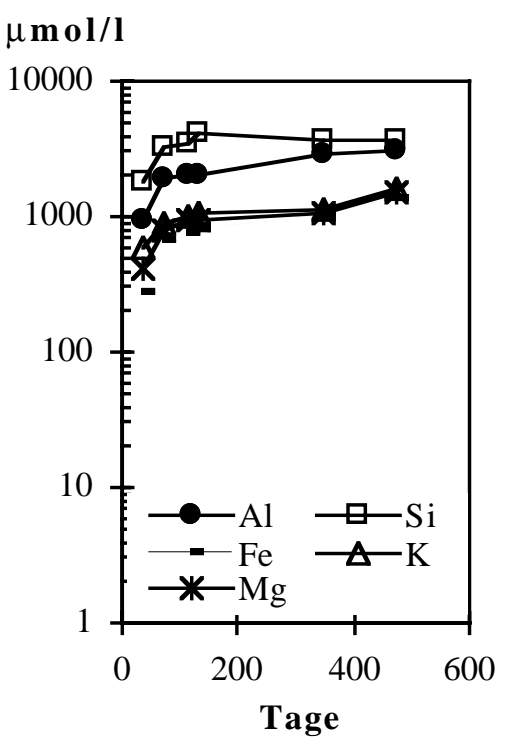

(b)

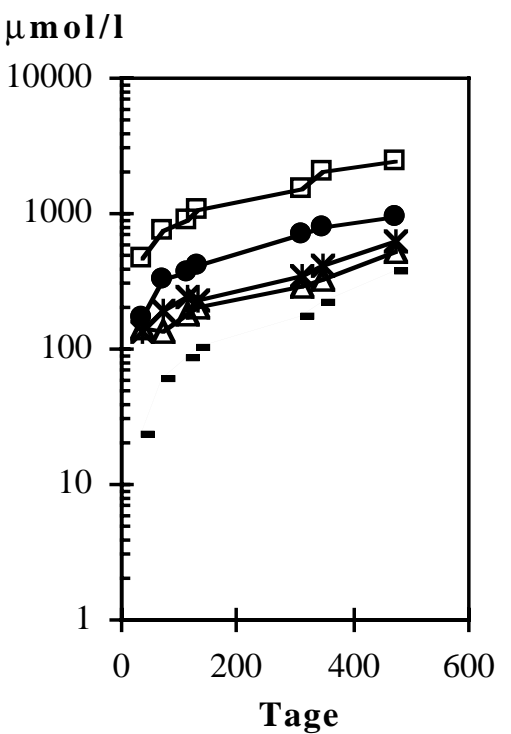

(c)

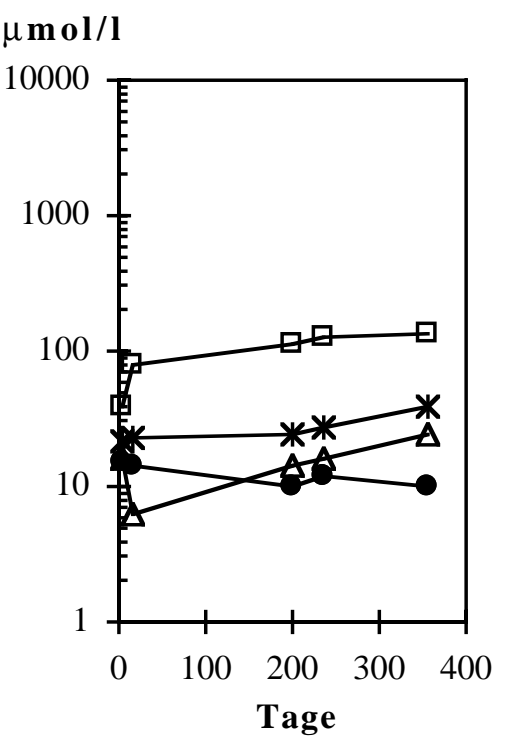

Abb. 6-20: Auflösung von Illit OECD in $\mathrm{H}_{2} \mathrm{SO}_{4}$-saurer Lösung bei pH 1 (a), pH 2 (b) und pH 3 (c) im Batch-Experiment ( vgl. Versuche 19, 22 und 29).

Der Illit OECD geht bei pH 1 sehr rasch in Lösung, die Lösungsgeschwindigkeit von $\mathrm{R}_{\mathrm{Si}}$ liegt in der Anfangsphase bei $\approx 1 \bullet 10^{-6}[\mathrm{~mol} /(\mathrm{g} \bullet \mathrm{h})]$. Man wird von annähernd kongruenter Auflösung sprechen können, muß aber im Auge behalten, daß die Molverhältnisse Si/Fe und $\mathrm{Si} / \mathrm{Mg}$ in der Lösung auf bevorzugtes Herauslösen dieser Ionen aus den Oktaederpositionen hindeuten. Bereits nach 75 Tagen nimmt die Lösungsgeschwindigkeit stark $\mathrm{ab}$, der $\mathrm{R}_{\mathrm{Si}}$-Wert sinkt im Zeitraum von 75 - 132 Tagen auf $\approx 3 \cdot 10^{-7}[\mathrm{~mol} /(\mathrm{g} \bullet \mathrm{h})]$ ab. Die Kurvenverläufe werden immer flacher (Abb. 6-20a). Höchstwahrscheinlich wird dies verursacht durch die langsame Annäherung an den Sättigungswert in bezug auf Illit. Ähnliches war schon beim Montmorillonit beobachtet worden. Bei pH 2 geht der Illit nach einer raschen Anfangsphase gleichmäßig in Lösung, das Si/Al-Molverhältnis schwankt zwischen 2 und 3. Alle anderen wesentlichen Gitterbausteine sind in der Lösung nachweisbar, das Molverhältniss Si/Mg läßt leicht 
bevorzugtes Herauslösen von $\mathrm{Mg}$ vermuten, beim $\mathrm{K}$ und $\mathrm{Fe}$ ist dies nicht genau zu ermittlen. Bei dem Versuch im pH-Bereich 3 - 4 zeigt die Si-Konzentration einen langsamen Anstieg, die Lösungsgeschwindigkeit $\mathrm{R}_{\mathrm{Si}}$ liegt bei $\approx 7 \bullet 10^{-9}[\mathrm{~mol} /(\mathrm{g} \bullet \mathrm{h})]$. Die Al-Konzentration scheint dagegen bei einem sehr niedrigen Wert von $\approx 10 \mu \mathrm{mol} \mathrm{Al} / \mathrm{l} \mathrm{zu}$ stagnieren. Bei ungestörter kongruenter Auflösung müßte die Al-Konzentration deutlich höher liegen. Simultan einsetzende Adsorptionsvorgänge könnten die Al-Konzentration kontrollieren.

Die Auflösungsversuche im $\mathrm{HNO}_{3}$-sauren Milieu sind in Abbildung 6-21 dargestellt.

(a)

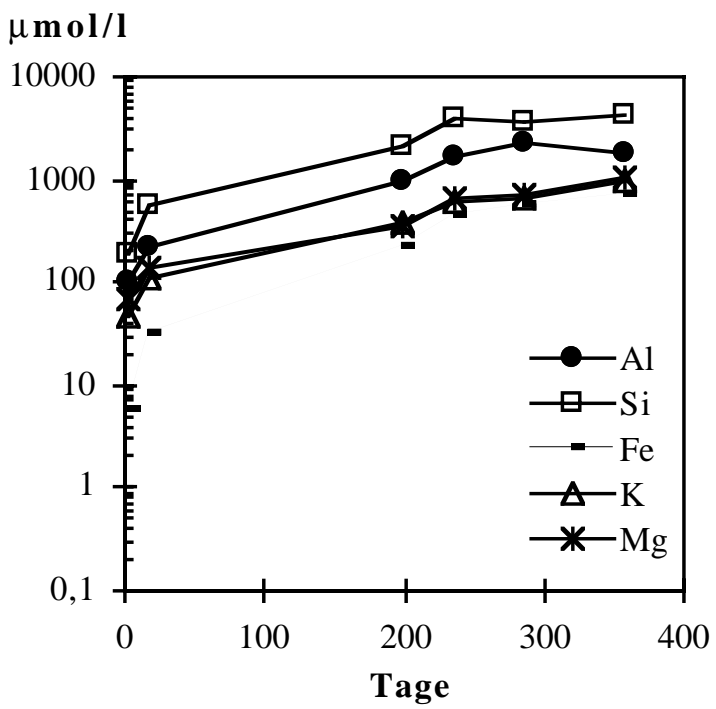

(b)

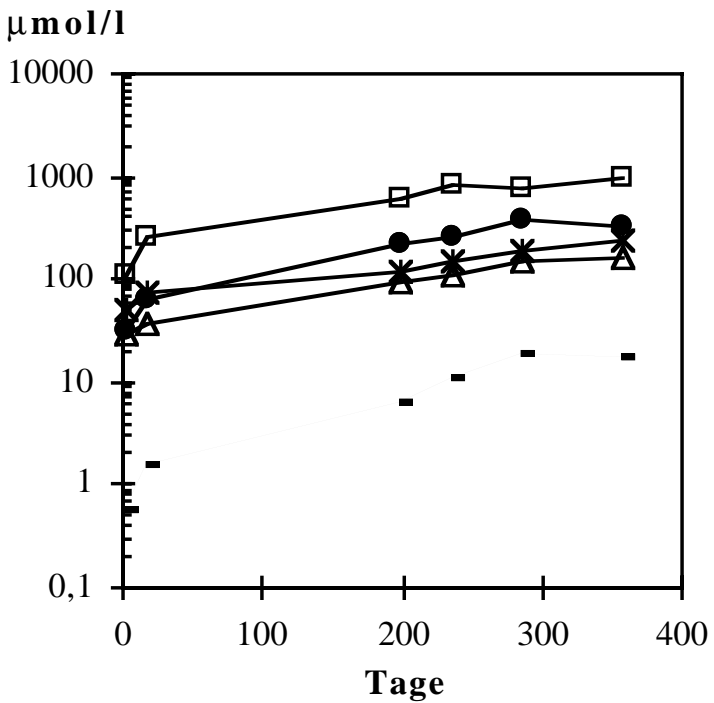

Abb. 6-21: Auflösung von Illit OECD in $\mathrm{HNO}_{3}$-saurer Lösung bei pH 1 (a) und pH 2 (b) im Batch-Experiment (vgl. Versuche 34 und 35).

In den Versuchen mit $\mathrm{HNO}_{3}$ sind die Auflösungsgeschwindigkeiten etwas geringer als bei den Versuchen in $\mathrm{H}_{2} \mathrm{SO}_{4}$-saurer Lösung. So wird bei $\mathrm{pH} 1$ auch nach einer Versuchszeit von 350 Tagen noch nicht der Konzentrationsbereich erreicht, in dem die Kurvenverläufe stark abflachen, sich die Konzentrationen in der Lösung also dem Sättigungswert in bezug auf Illit nähern. Die einzelnen Elemente zeigen stetigen, fast parallel verlaufenden Anstieg. Dies läßt eine annähernd kongruente Auflösung vermuten. Ähnlich ist der Kurvenverlauf bei pH 2 zu interpretieren.

Interessant ist der Reaktionsverlauf in $\mathrm{C}_{6} \mathrm{H}_{8} \mathrm{O}_{7}$-saurem Milieu (Abb. 6-22). Bei diesem Versuch begann der $\mathrm{pH}$ langsam zu höheren $\mathrm{pH}$-Werten zu driften. Während im $\mathrm{pH}$-Bereich 2,5 - 3,2 die Konzentration der einzelnen Ionen stetig zunahm, kam es im pH-Bereich 4,9 - 5,4 beim Al und $\mathrm{Fe}$ zu einer Absenkung der Konzentrationen. Die Konzentration der anderen Elemente stagnierte in diesem hohen $\mathrm{pH}$-Bereich, was sicher mit der stark abnehmenden Auflösungsgeschwindigkeit mit steigendem pH-Wert zusammenhängt. 


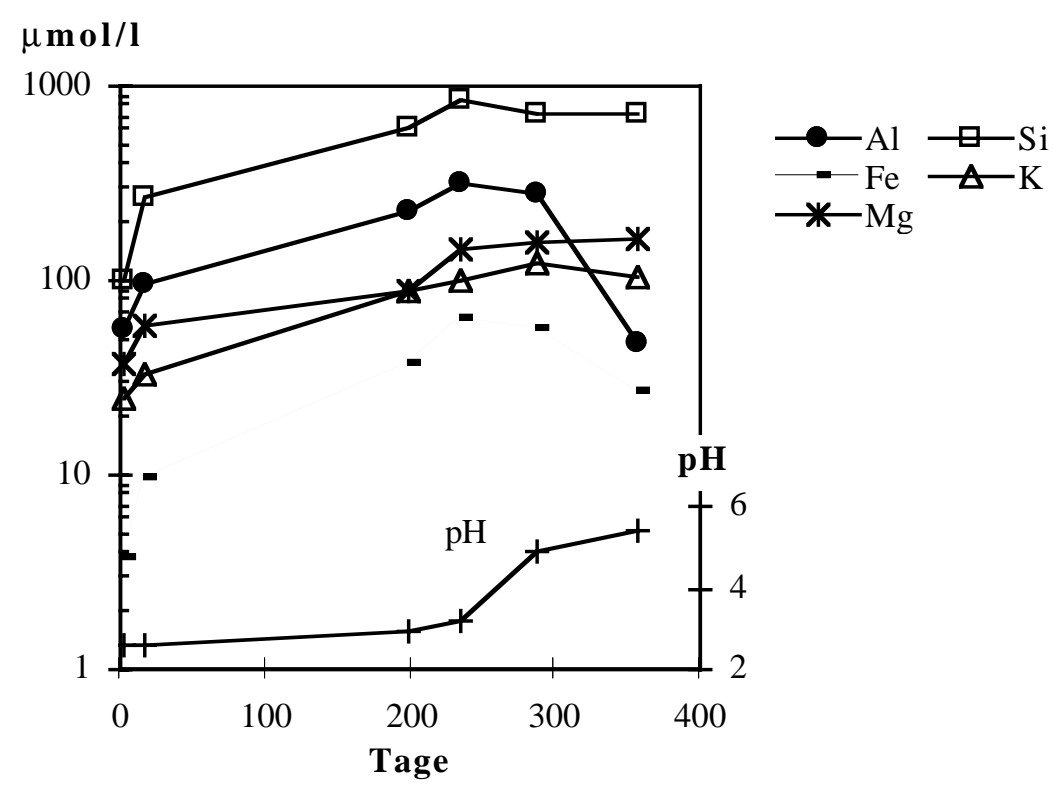

Abb. 6-22: Auflösung von Illit OECD in $\mathrm{C}_{6} \mathrm{H}_{8} \mathrm{O}_{7}$-saurer Lösung im Batch-Experiment (vgl. Versuch 43).

Der deutliche Konzentrationsabfall im pH-Bereich 4,9 - 5,4 für Al und Fe weist in Richtung Ausfällung von Oxidhydraten oder Hydroxiden. Als Vergleich sind in Tabelle 6-18 entsprechende Daten zusammengestellt.

Tab. 6-18: Zusammenstellung der Sättigungskonzentrationen einiger Fe- und Al-Hydroxide und Oxidhydrate bei ausgewählten pH-Werten. Formeln für Sättigungskonzentrationen nach Lindsay (1979), 1: nach Morel \& Hering (1993).

\begin{tabular}{cccc}
\hline $\mathrm{pH}$ & $\begin{array}{c}\mathrm{Al}(\mathrm{OH})_{3} \text { Gibbsit } \\
\lg \left(\mathrm{Al}^{3+}\right)=8,04-3 \bullet p H\end{array}$ & $\begin{array}{c}\mathrm{Al}(\mathrm{OH})_{3} \text { amorph } \\
\lg \left(\mathrm{Al}^{3+}\right)=9,66-3 \bullet p H\end{array}$ & $\begin{array}{c}\mathrm{Fe}(\mathrm{OH})_{3} \text { amorph } \\
\sum \mathrm{Fe}^{1}\end{array}$ \\
\hline \hline & $\mathrm{Al}[\mathrm{mol} / \mathrm{l}]$ & $\mathrm{Al}[\mathrm{mol} / \mathrm{l}]$ & $\mathrm{Fe}[\mathrm{mol} / \mathrm{l}]$ \\
\hline 2,6 & 1,74 & 1,86 & $9,6 \bullet 10^{-5}$ \\
2,9 & 0,22 & 0,96 & $2,3 \bullet 10^{-5}$ \\
3,2 & 0,028 & 0,06 & $6,4 \bullet 10^{-6}$ \\
4,9 & $2,2 \bullet 10^{-7}$ & $9,1 \bullet 10^{-6}$ & $4,1 \bullet 10^{-8}$ \\
5,4 & $6,9 \bullet 10^{-9}$ & $2,9 \bullet 10^{-7}$ & $1,3 \bullet 10^{-8}$ \\
\hline
\end{tabular}

Bei der Illitprobe H-35 erkennt man in allen Batch-Experimenten, vor allem bei $\mathrm{pH} 1$ und $\mathrm{pH} 2$, die rasche Auflösung der Fe-Oxidhydrat-Überzüge in der Anfangsphase (Abb. 6-23). Die Kurvenverläufe auch der anderen Elemente scheinen dann überwiegend durch die Auflösung des Illites kontrolliert zu werden, der ja als Hauptkomonente in dieser multimineralischen Probe vorliegt. Wie schon beim Illit OECD erwähnt, weisen die, verglichen mit dem Festkörper, zu 
(a)

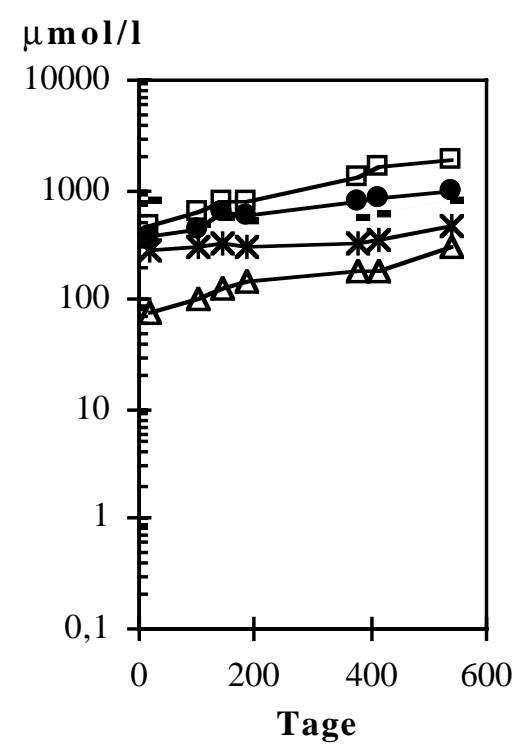

(d)

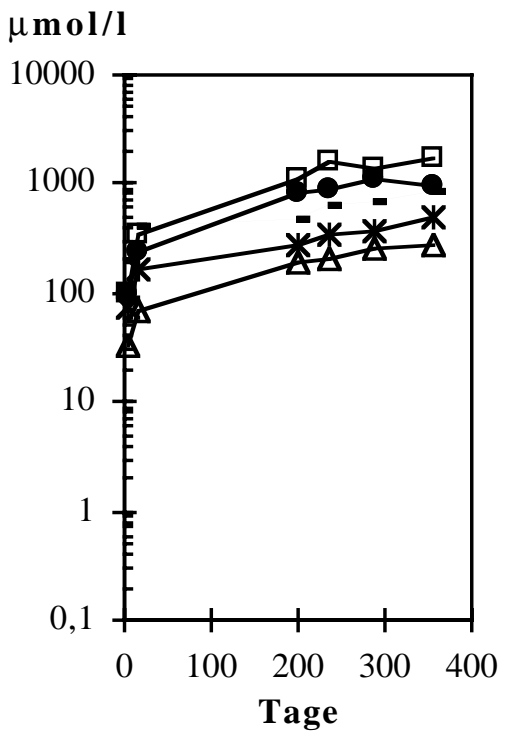

(b)

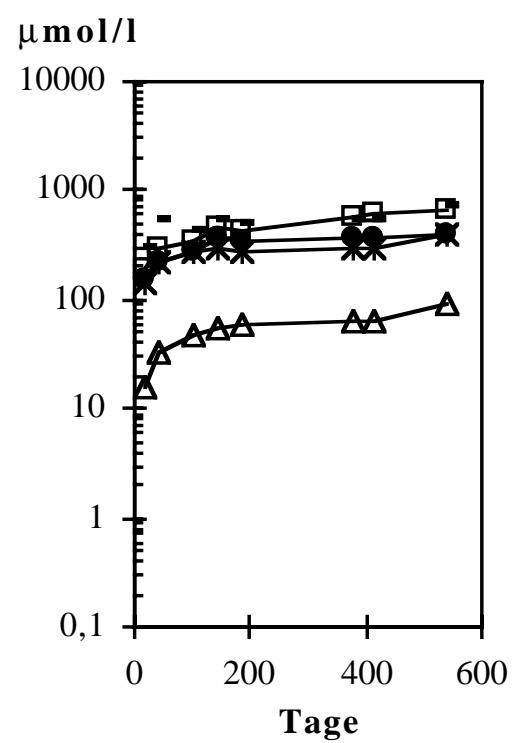

(e)

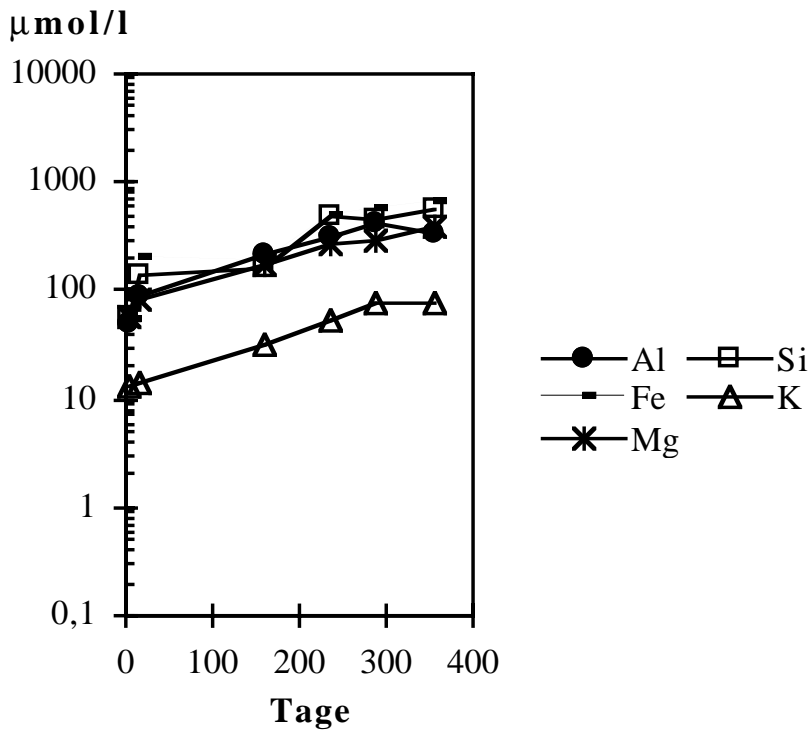

Abb. 6-23: Auflösung von Illit $\mathrm{H}$-35 in $\mathrm{H}_{2} \mathrm{SO}_{4}$-saurer Lösung bei pH 1 (a), pH 2 (b) und pH 3

(c) sowie in $\mathrm{HNO}_{3}$-saurer Lösung bei $\mathrm{pH} \mathrm{l}(\mathrm{d})$ und $\mathrm{pH} 2$ (e) in Batch-Experimenten (vgl. Versuche 10, 11, 28, 31 und 32). 
niedrigen Molverhältnisse in den Lösungen wohl vor allem auf bevorzugtes Herauslösen der Gitterbausteine Fe und Mg hin (Tab. 6-19). Feigenbaum \& Shainberg (1975) beschreiben dieses Phänomen schon bei ihren Auflösungsversuchen mit „Fithian illite”. Leider lassen sich die Untersuchungsergebnisse wegen der sehr unterschiedlichen Versuchsbedingungen nicht direkt miteinander vergleichen. Bei dem Versuch bei pH 3 scheinen Adsorptionseffekte die niedrigen Al-Konzentrationen zu kontrollieren.

Tab. 6-19: Element-Molverhältnisse aus der Auflösung der Illitprobe $\mathrm{H}$-35 in $\mathrm{H}_{2} \mathrm{SO}_{4}$ - und $\mathrm{HNO}_{3}$-saurer Lösung im Batch-Experiment (vgl. Versuch 10, 11, 28, 31 und 32).

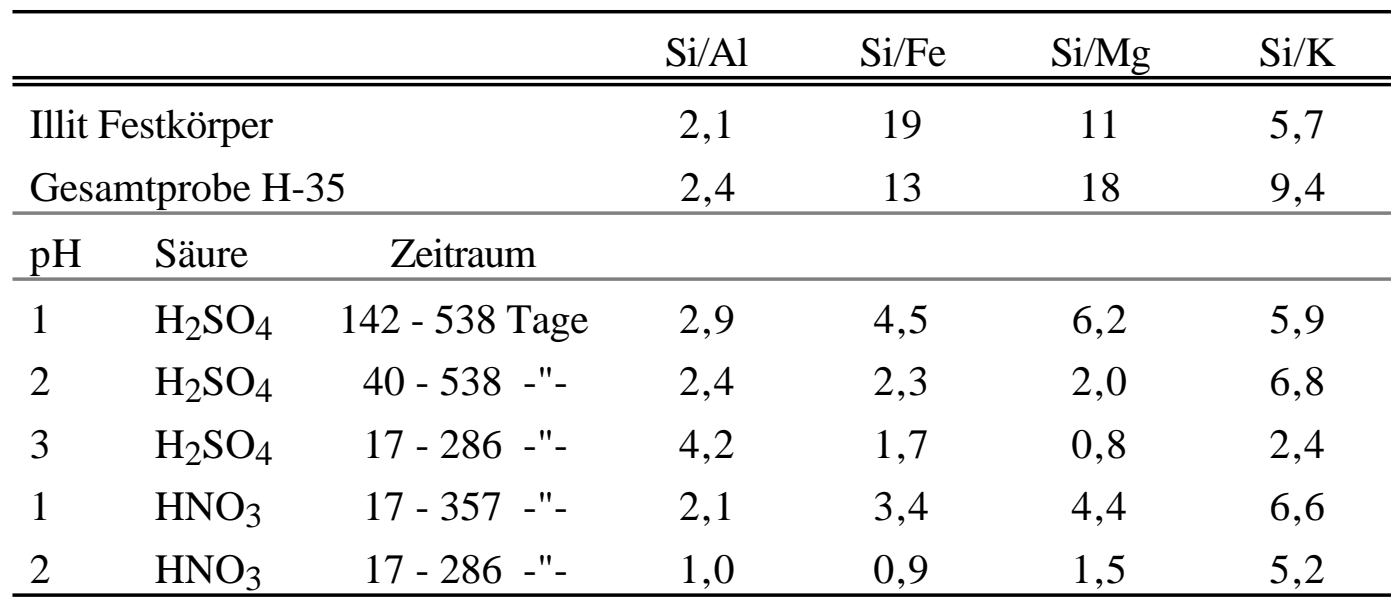

Beim Illit H-35 können ähnlich wie beim Kaolinit zunehmende Kantenrundungen, Oberflächenrauhigkeiten und Verringerung der Partikelgröße beobachtet werden (Abb. 6-24).

Daß bei den Auflösungsversuchen auch andere Mineralkomponenten der Probe Illit H-35 mit in Lösung gehen können, ist in Abbildung 6-25 zu erkennen. Bei dem in der FEM-Aufnahme gezeigten Mineralkorn handelt es sich nicht um Illit, sondern vermutlich um einen Feldspat, der deutliche Korrosionsspuren zeigt. Die Ausbildung dieser Strukturen scheint für Feldspäte typisch zu sein und ist u.a. bei Hochella \& Banfield (1995) dokumentiert. 


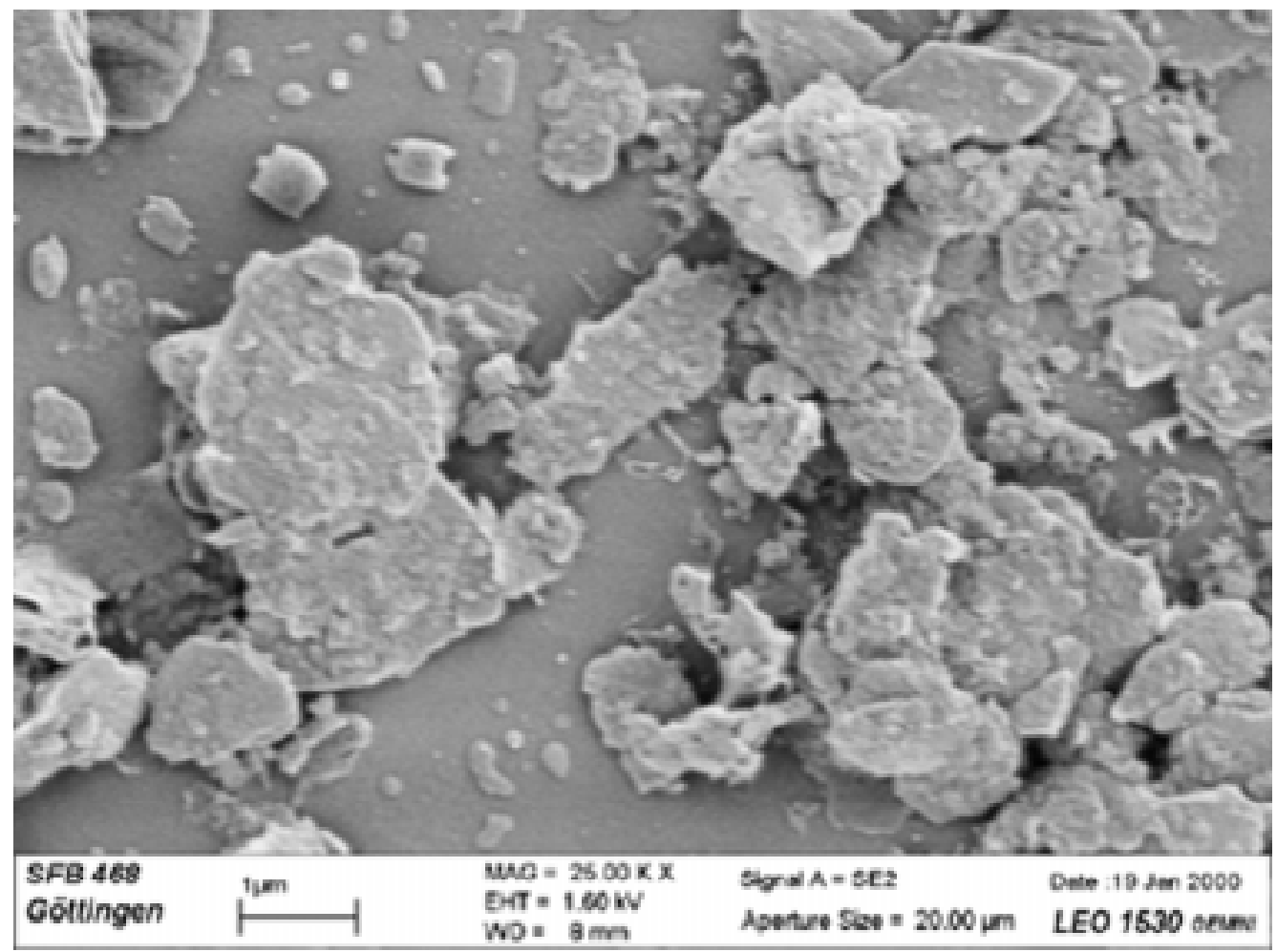

Abb. 6-24: FEM-Aufnahme der Illitprobe $\mathrm{H}$-35 nach Behandlung mit $\mathrm{H}_{2} \mathrm{SO}_{4}$-saurer Lösung bei pH 1 im Batch-Experiment.

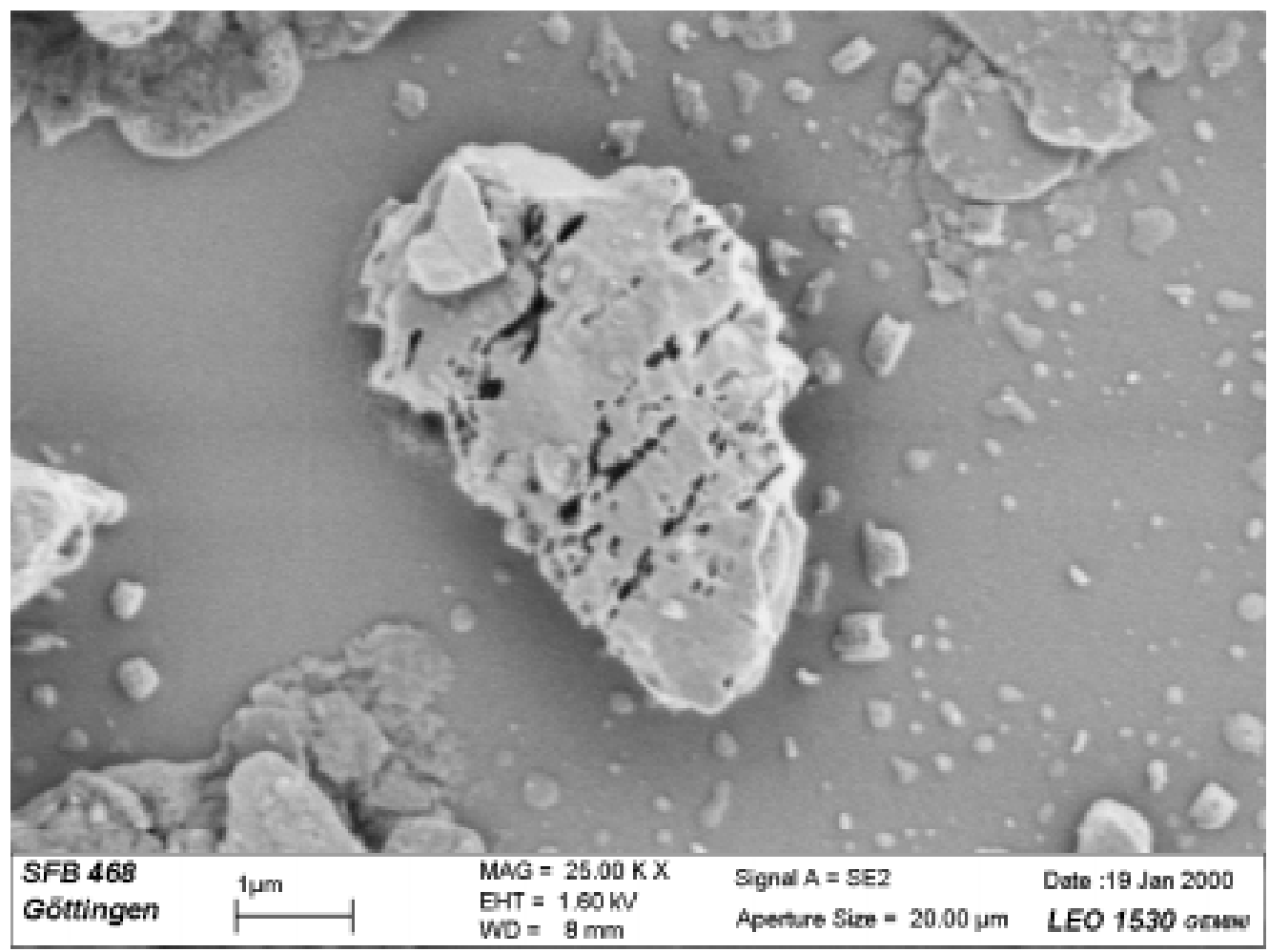

Abb. 6-25: FEM-Aufnahme von Lösungsspuren -vermutlich an einem Feldspat- der Illitprobe H-35 nach Behandlung mit $\mathrm{H}_{2} \mathrm{SO}_{4}$-saurer Lösung bei $\mathrm{pH} 1 \mathrm{im}$ Batch-Experiment. 
Zusammenfassend läßt sich zum Auflösungsverhalten des Serizits und der Illite folgendes sagen:

- Sie gehen bei pH 1 und pH 2 annähernd kongruent in Lösung, wenn man das Si/AlMolverhältnis in der Lösung betrachtet. Die anderen Gitterbausteine $\mathrm{Fe}, \mathrm{Mg}$ und $\mathrm{K}$ gehen im allgemeinen leicht bevorzugt in Lösung. Dies ist erkennbar an den zum Teil zu niedrigen Molverhältnissen in der Lösung im Vergleich zu den Werten im Festkörper.

- Bei pH 3 scheinen Adsorptionsvorgänge die Al-Konzentration in der Lösung zu beeinflussen.

- Bei $\mathrm{pH} \geq 4$ sind Ausfällungsreaktionen für die Al- und Fe-Konzentrationen verantwortlich. Die Löslichkeiten von Al- und Fe-Hydroxiden werden überschritten.

- Die Auflösungsgeschwindigkeiten, vor allem bezogen auf $\mathrm{R}_{\mathrm{Si}}$, nehmen mit steigendem $\mathrm{pH}$ Wert deutlich ab. Aus dieser Beziehung lassen sich Reaktionsordnungen ermitteln.

Die aus den Auflösungsraten $\mathrm{R}_{\mathrm{Si}}$ der Durchfluß-Experimente abgeleiteten Reaktionsordnungen sind in Abbildung 6-26 zusammengefaßt. Bei den Batch-Experimenten ist die Ermittlung von Reaktionsordnungen aus den $\mathrm{R}_{\mathrm{Si}}$-Werten erschwert, da die Auflösungsgeschwindigkeiten sich zum Teil im Verlauf der Versuche änderten.

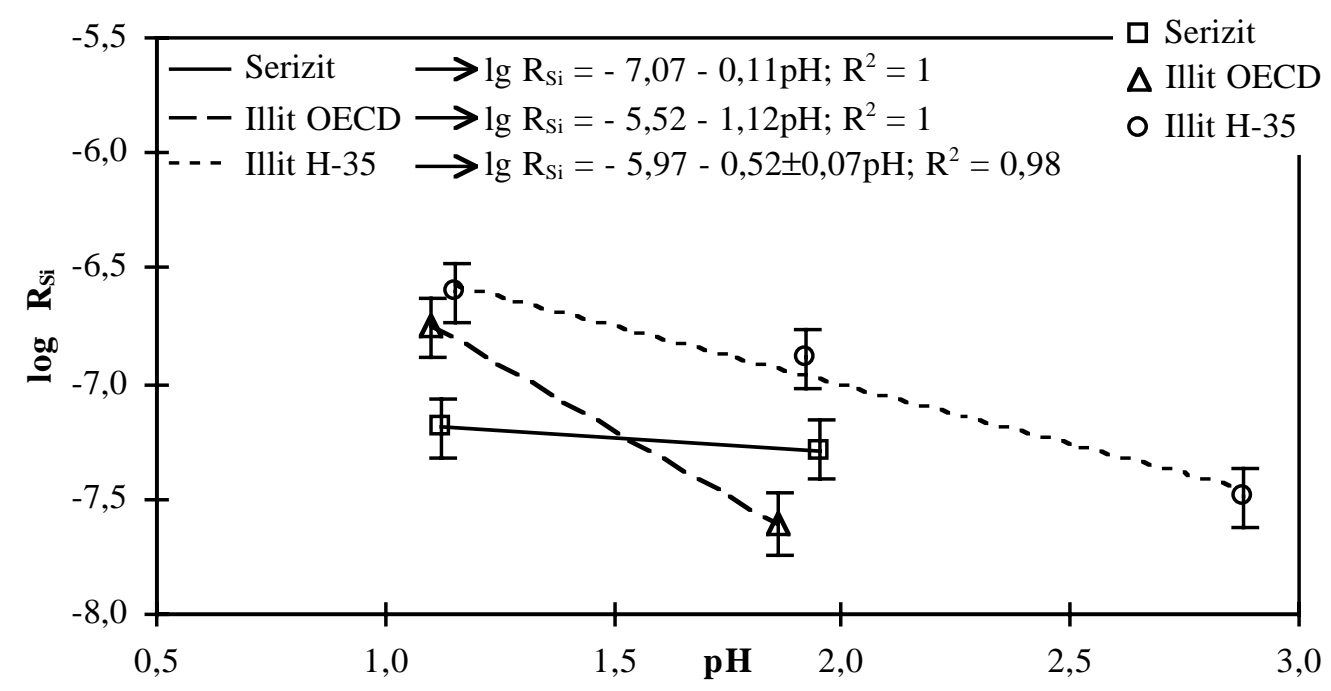

Abb. 6-26: Logarithmus der Auflösungsrate von Si $\left(R_{S i}\right)$ in Abhängigkeit vom pH-Wert für Durchfluß-Experimente mit Serizit, Illit OECD und Illit $\mathrm{H}$-35 in $\mathrm{H}_{2} \mathrm{SO}_{4}$-saurer Lösung. Aus der Steigung der Geraden ergibt sich jeweils die Reaktionsordnung $n$. 


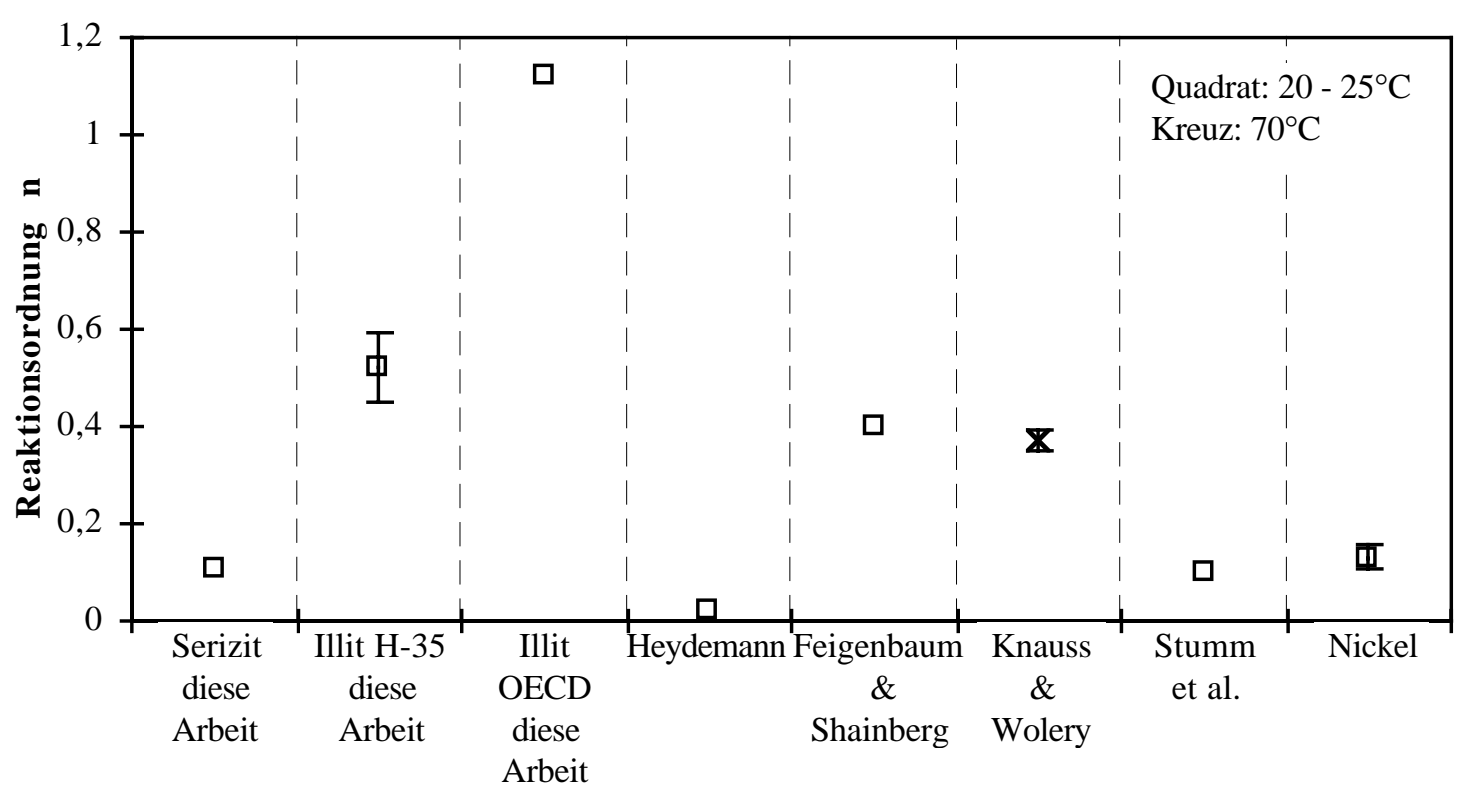

Abb. 6-27: Vergleich der ermittelten Reaktionsordnungen $n$ mit Daten aus der Literatur (vgl. auch Tab. 6-20).

Die aus den Auflösungsraten $\mathrm{R}_{\mathrm{Si}}$ abgeleiteten Reaktionsordnungen $\mathrm{n}$ zeigen für die in dieser Arbeit untersuchten Minerale eine große Streuung. Läßt man bei einem Vergleich mit Literaturdaten den recht hohen Wert für den Illit OECD außer acht, so zeigt sich eine gute Übereinstimmung der Reaktionsordnung für den Illit H-35 mit denen für Illit von Feigenbaum \& Shainberg (1975) bzw. für einen Muskovit bei $70^{\circ} \mathrm{C}$ von Knauss \& Wolery (1989). Die in dieser Arbeit ermittelte Reaktionsordnung für Serizit ist vergleichbar mit der von Stumm et al. (1987) bzw. Nickel (1973) für Muskovit und der von Heydemann (1966) für Illit.

Tab. 6-20: Reaktionsordnungen n für protonenkontrollierte Auflösung von Serizit, Illit und Muskovit (a: $n$ basiert auf 2 Datenpunkten).

\begin{tabular}{llcccccl}
\hline Mineral & Lösung & Methode & $\begin{array}{c}\mathrm{pH}- \\
\text { Bereich }\end{array}$ & $\begin{array}{c}\text { Fehler (n) } \\
( \pm)\end{array}$ & $\begin{array}{c}\text { Temp. } \\
{\left[{ }^{\circ} \mathrm{C}\right]}\end{array}$ & Autor \\
\hline \hline Serizit & $\mathrm{H}_{2} \mathrm{SO}_{4}$ & Durchfluß & $1-2$ & 0,11 & $\mathrm{a}$ & 25 & diese Arbeit \\
Illit $\mathrm{H}-35$ & $\mathrm{H}_{2} \mathrm{SO}_{4}$ & Durchfluß & $1-3$ & 0,52 & 0,07 & 25 & diese Arbeit \\
Illit OECD & $\mathrm{H}_{2} \mathrm{SO}_{4}$ & Durchfluß & $1-2$ & 1,12 & $\mathrm{a}$ & 25 & diese Arbeit \\
Illit & $\mathrm{H}_{2} \mathrm{SO}_{4}$ & Mixed flow & $3-6$ & 0,02 & $\mathrm{a}$ & 20 & Heydemann (1966) \\
Illit & $\mathrm{HCl} / \mathrm{CaCl}_{2}$ & Batch & $3-7,5$ & 0,4 & $\mathrm{a}$ & 25 & Feigenbaum \& \\
& & & & & & & Shainberg (1975) \\
Muskovit & $\mathrm{Puffer}$ & Durchfluß & $2,1-5,0$ & 0,37 & 0,02 & 70 & Knauss \& \\
& & & & & & & Wolery (1989) \\
Muskovit & $0,1 \mathrm{M} \mathrm{NaNO}_{3}$ & Batch & $3-5$ & 0,1 & $\mathrm{a}$ & 25 & Stumm et al. (1987) \\
Muskovit & $\mathrm{HCl}_{1} \mathrm{CO}_{2}$ & Stirred flow & $0,2-5,6$ & 0,13 & 0,02 & 25 & Nickel (1973) \\
\hline
\end{tabular}




\subsection{Talk}

Talk wurde als Modellsubstanz für trioktaedrische Dreischichtminerale mit $\mathrm{Mg}$ in den Oktaederpositionen ausgewählt. Im $\mathrm{pH}$-Bereich $\geq 4$ sollte es beim Auflösungsprozeß des Talks im Gegensatz zu Al-haltigen Schichtsilikaten zu keinen Ausfällungen von Mg-Hydroxiden kommen, da unter den gegebenen Bedingungen die entsprechenden Sättigungskonzentrationen nicht überschritten werden.

Die chemischen und mineralogischen Daten der Talkprobe sind aus Tabelle 5-1 zu ersehen. Das Probenmaterial lag in feingemahlener Form vor. Durch den Mahlprozeß waren viele größere Talkblättchen zerbrochen worden, wodurch es in dem Probenmaterial Bruchflächen und Defektstellen an den Partikeloberflächen gab. Trotz einer Korngrößentrennung durch Sedimentation enthält die hier verwendete Fraktion von 2 - $63 \mu \mathrm{m} \varnothing$ noch viele feinere Partikel, die an den gröberen anhaften. Gut zu erkennen ist dies in der Abbildung 6-28A. In Abbildung 6-28B sind als ein weiteres Merkmal deutliche Stufen im Kantenbereich der Partikel zu erkennen.

Exemplarisch für die Vorgänge, die bei der Auflösung des Talks zu beobachten sind, werden hier nur die Ergebnisse der beiden Durchfluß-Experimente gezeigt (Abb. 6-29). Bei dieser Versuchsanordnung werden die in der Anfangsphase in Lösung gehenden Elemente fortlaufend aus dem System entfernt und können nicht wie in den Batch-Experimenten akkumulieren.

Deutlich erkennbar in beiden Versuchen sind die zunächst sehr hohen Konzentrationen von Si und $\mathrm{Mg}$ in der Lösung. Sie resultieren aus der Auflösung der vielen winzigen Partikel und der an den Bruchflächen erzeugten Defektstellen. Im Versuch mit $\mathrm{HNO}_{3}$-saurer Lösung liegen die in der Lösung gemessenen Mg-Konzentrationen in der Anfangsphase sogar höher als die SiKonzentrationen, während im Festkörper das Si/Mg-Molverhältnis bei $\approx 1,4$ liegt. Nach Entfernen dieser leichter löslichen Partikel und/oder Phasen erreicht man in beiden Versuchen nach 50 - 100 Tagen ,steady state"-Bedingungen. Im Versuch mit $\mathrm{H}_{2} \mathrm{SO}_{4}$-saurer Lösung deutet das Si/Mg-Molverhältnis in der Lösung mit $\approx 2,5$ auf annähernd kongruente Auflösung hin. Bei dem Versuch mit $\mathrm{HNO}_{3}$-saurer Lösung liegt das Si/Mg-Molverhältnis in der Lösung bei $\approx 10$. Noch ist keine überzeugende Erklärung für dieses Phänomen gefunden worden. 
Ergebnisse und Diskussion
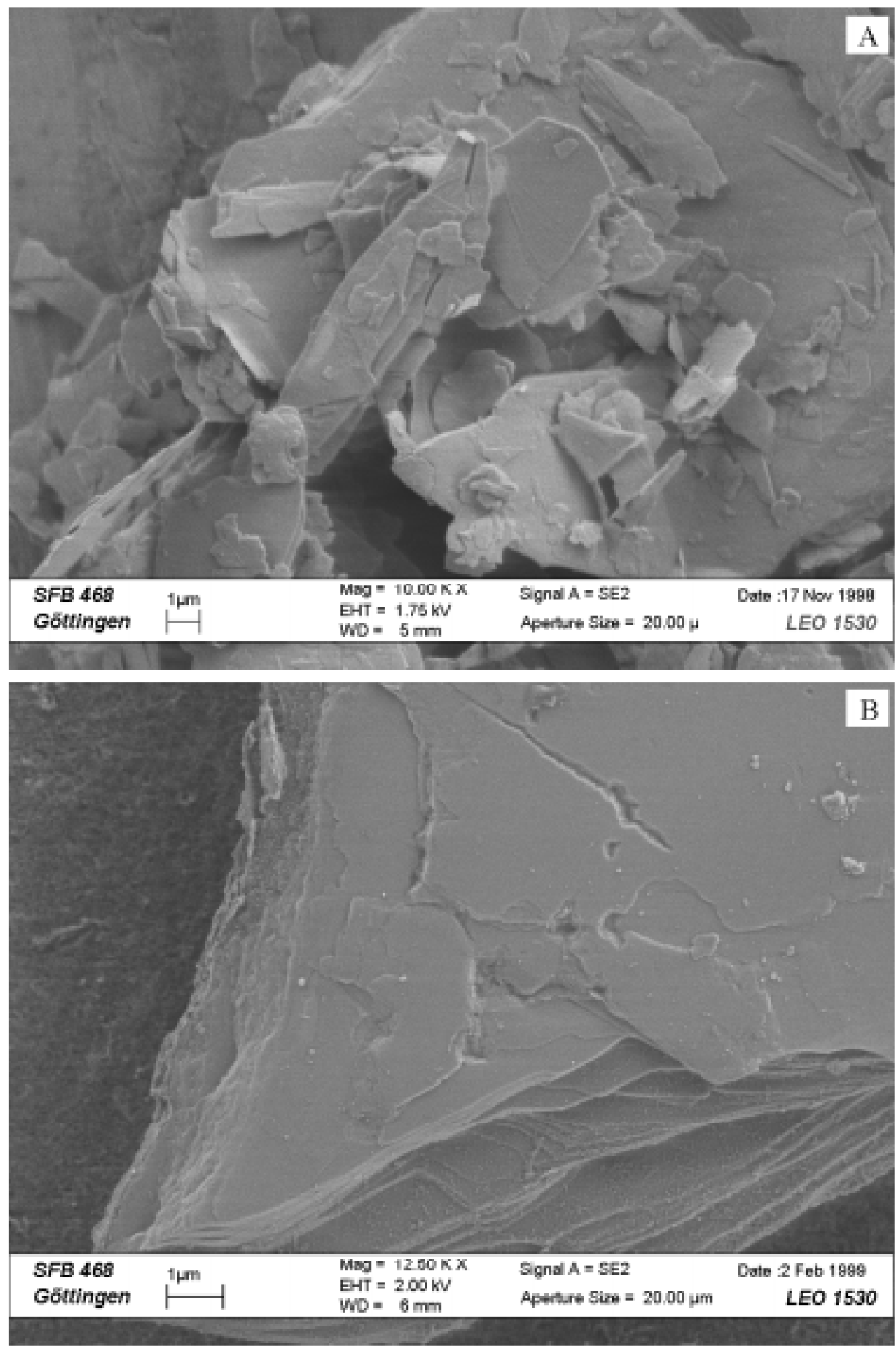

Abb. 6-28: FEM-Aufnahme des unbehandelten Talks. 
(a)

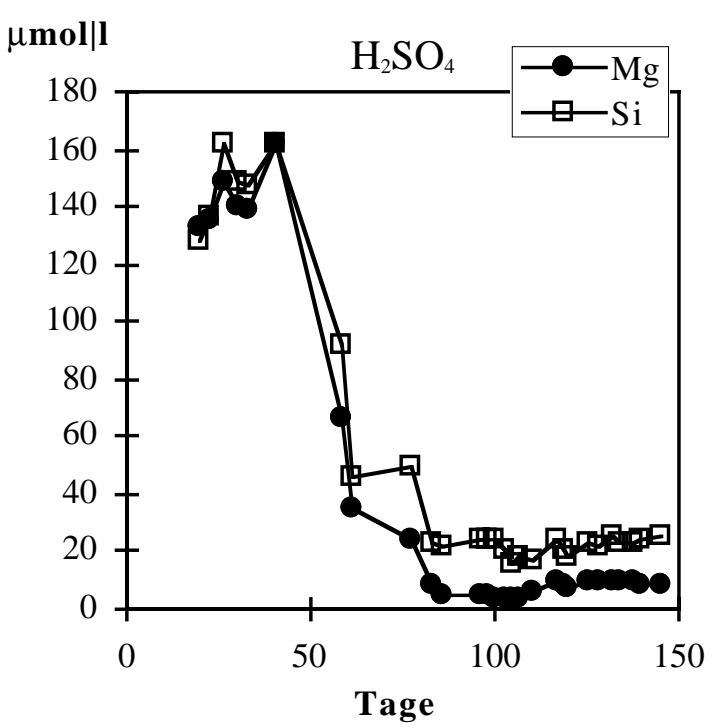

(b)

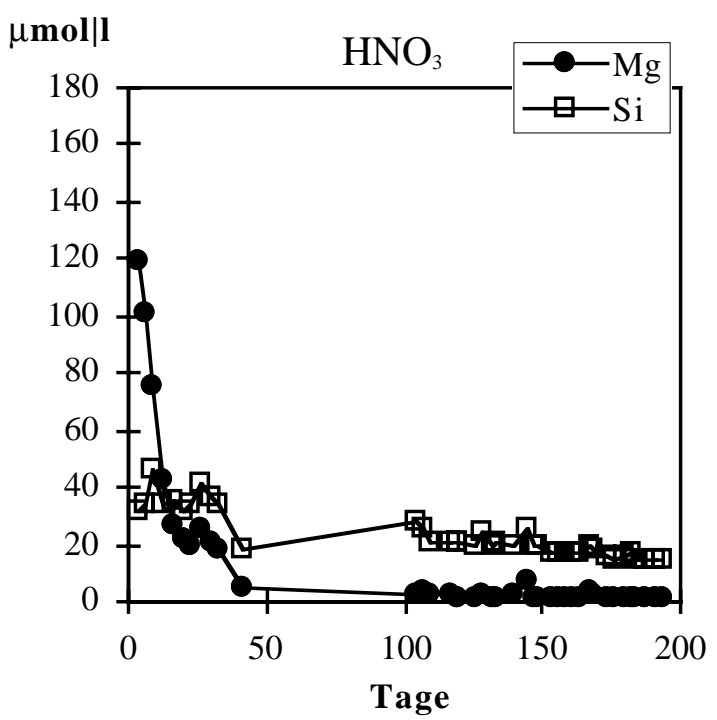

Abb. 6-29: Auflösung von Talk in $\mathrm{H}_{2} \mathrm{SO}_{4^{-}}$(a) und $\mathrm{HNO}_{3}$-saurer Lösung (b) bei pH 1 im Durchfluß-Experiment (vgl. Versuche XI (a), XIII (b)).

In Abbildung 6-30 sind Talkpartikel nach Behandlung in dem Durchfluß-Experiment mit $\mathrm{HNO}_{3}$-saurer Lösung gezeigt. Weiterhin deutlich zu erkennen sind die Stufenstrukturen im Kantenbereich der Partikel. Darüber hinaus scheint die Partikelgröße tendenziell kleiner geworden zu sein.

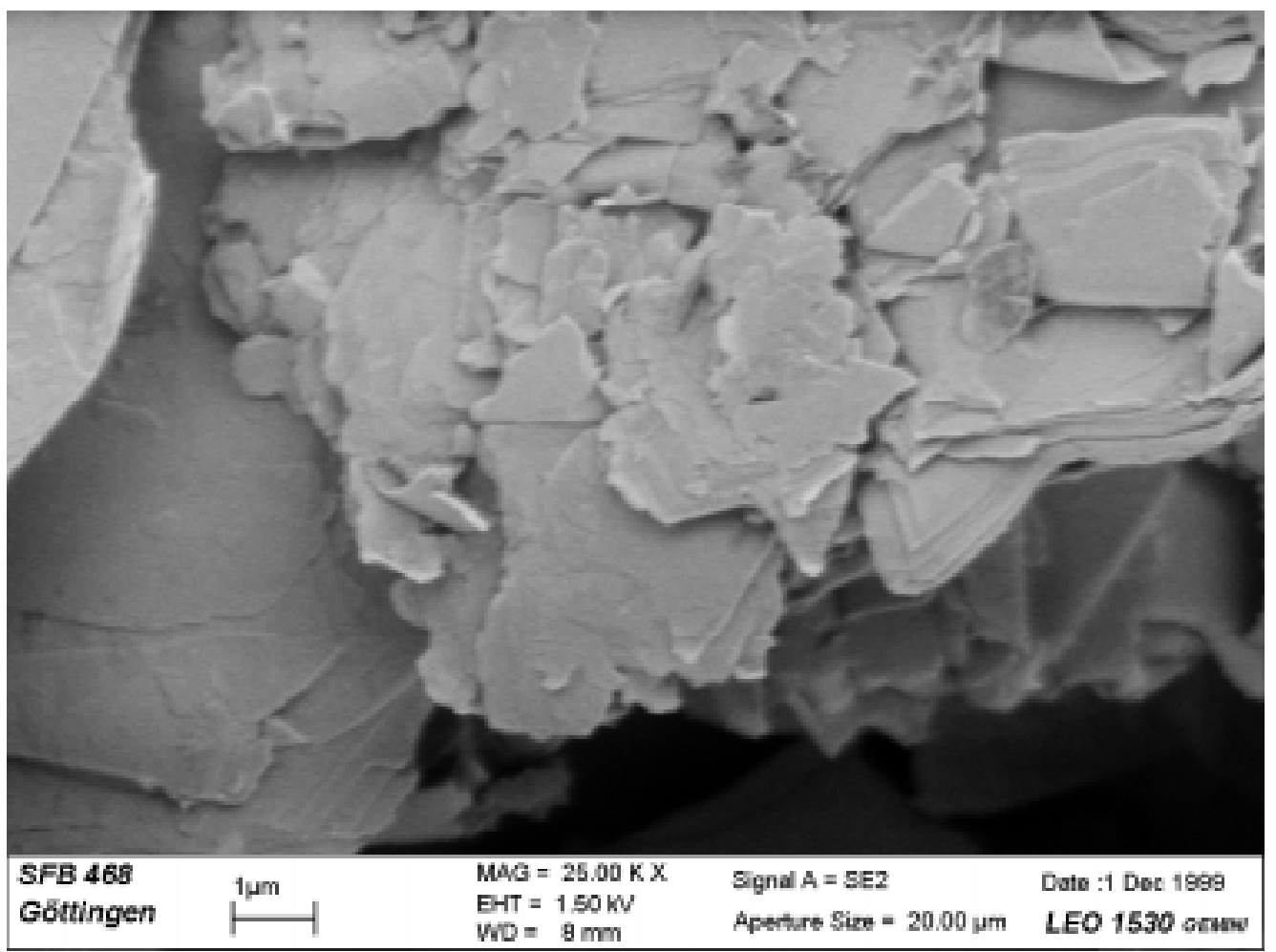

Abb. 6-30: FEM-Aufnahme des Talks nach Behandlung mit $\mathrm{HNO}_{3}$-saurer Lösung bei pH $1 \mathrm{im}$ Durchfluß-Experiment. 
Die Anzahl der mit dem Talk durchgeführten Experimente ist verglichen mit den anderen Mineralen sehr gering. Als sich die durch den Mahlprozeß beschriebenen Probleme abzeichneten, wurde zugunsten der anderen Minerale auf die Durchführung zahlreicher Experimente verzichtet. Die Interpretierbarkeit der Befunde wird durch die Art der Vorbehandlung deutlich erschwert. 


\subsection{Tonminerale und Porenlösung}

Es wurden noch eine Reihe von Versuchen durchgeführt, die Hinweise über Reaktionsprozesse zwischen Tonmineralen und Porenlösung liefern sollten. Als Porenlösung stand die Porenlösung-I von einem Bodenprofil unter Buche aus dem Solling vom Tiefenbereich 0 - 15 cm zur Verfügung (Tab. A-2).

Bei allen Versuchen kam es zu einer Drift des Anfangs-pH-Wertes von 3,5 zu höheren pHWerten. Die Auflösungsreaktionen wurden im Verlauf der Versuche durch andere Prozesse überlagert, die nur unter höheren $\mathrm{pH}$-Bedingungen stattfinden können.

Bei dem Versuch Porenlösung-I-Kaolinit sollten durch Auflösung des Minerals nur die Si- und Al-Konzentrationen in der Lösung ansteigen. Mit zunehmender Versuchsdauer beobachtet man auch eine leichte Zunahme der Si-Konzentration von 362 auf $429 \mu \mathrm{mol} \mathrm{Si} / 1$ (Abb. 6-31a), also tatsächlich einen Hinweis auf die Auflösung von Kaolinitpartikeln. Die Al- und FeKonzentrationen nehmen aber in der Lösung mit steigendem pH-Wert von 3,5 bis 5,7 ab und zwar von 20 auf $2 \mu \mathrm{mol} \mathrm{Al/1} \mathrm{bzw.} \mathrm{von} \mathrm{6,3} \mathrm{auf} \mathrm{1,5} \mu \mathrm{mol} \mathrm{Fe/l.} \mathrm{Dies} \mathrm{muß} \mathrm{auf} \mathrm{die} \mathrm{Ausfällung} \mathrm{von}$ Al- und Fe-Hydroxiden zurückgeführt werden. Allein die Löslichkeitsprodukte dieser Phasen kontrollieren in diesem pH-Bereich die Al- und Fe-Konzentration (vgl. Tab. 6-18).

Der Reaktionsablauf bei dem Versuch Porenlösung-I-Montmorillonit ist aus Abbildung 6-31b zu ersehen. Der leichte Anstieg der Si-Konzentration von 362 auf $465 \mu \mathrm{mol} \mathrm{Si} / 1$ deutet daraufhin, daß Montmorillonit in Lösung geht. Der zu Beginn beobachtete Anstieg der NaKonzentration in der Lösung resultiert aus Ionenaustauschreaktionen zwischen $\mathrm{Na}^{+}$im Zwischenschichtraum des Montmorillonits und $\mathrm{H}^{+}$-Ionen aus der zunächst schwach sauren Lösung. Nur etwa die Hälfte des Na aus dem Zwischenschichtraum wurde dabei ausgetauscht. Das Verhalten der Elemente $\mathrm{Al}, \mathrm{Fe}$ und Mg deutet wieder auf Prozesse hin, die parallel zum Auflösungsvorgang stattfinden. Die erste starke Konzentrationserniedrigung aller drei Elemente ist sicher auf Adsorptions-/Ionenaustauschreaktionen zurückzuführen. Die maximale Ionenaustauschkapazität der eingesetzten Montmorillonitprobe $(\approx 58 \mu \mathrm{mol} \mathrm{M}+/ 69 \mathrm{mg})$ reicht aus, um die aus der Porenlösung-I stammenden Ionen und die durch den Auflösungsprozeß zusätzlich in die Lösung gelangten zwei- und dreiwertigen Kationen zu fixieren. Ob die Al- und Fe- Konzentrationen außerdem noch beeinflußt werden durch Ausfällungsmechanismen ist schwer zu entscheiden. 
(a)

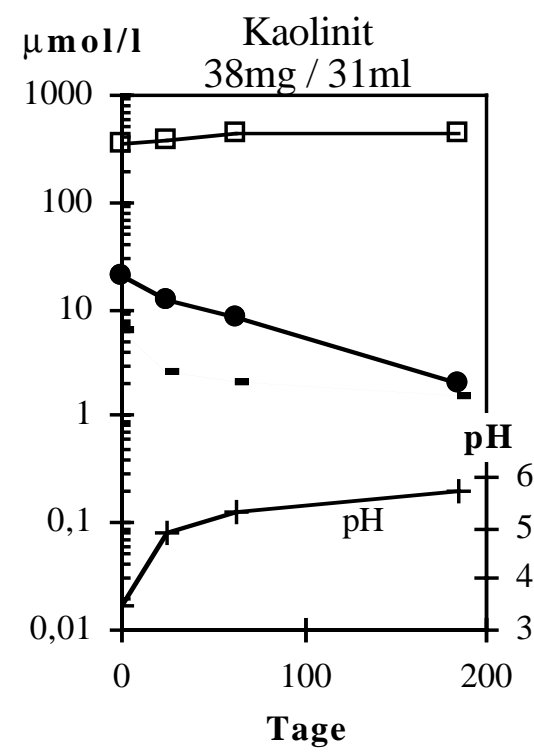

(b)

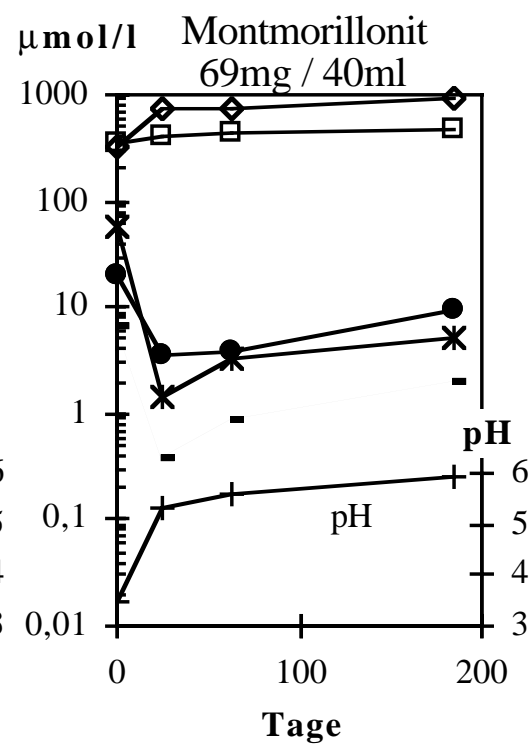

(c)

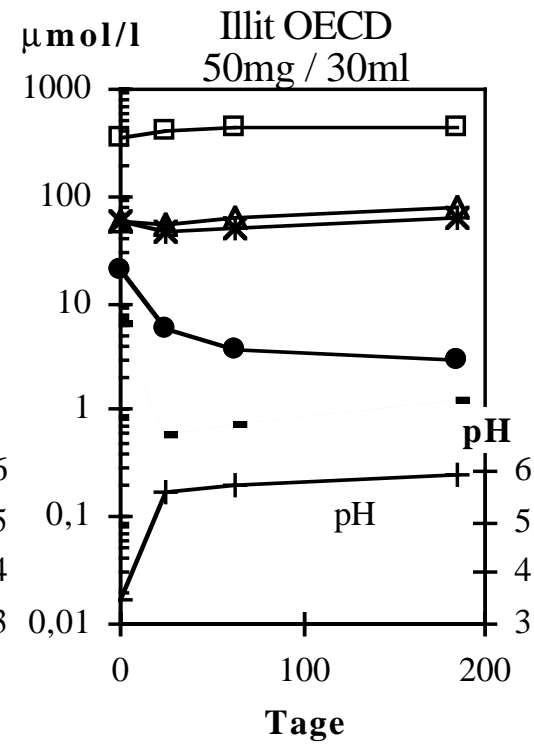

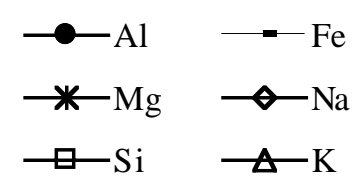

Abb. 6-31: Auflösung von Kaolinit (a), Montmorillonit (b) und Illit OECD (c) in Porenlösung-I vom Solling (vgl. Versuche 47, 45 und 48).

Der Reaktionsablauf beim Versuch Porenlösung-I-Illit OECD ist aus Abbildung 6-31c abzuleiten. Der leichte Anstieg der Si- und K-Konzentrationen deutet auf Auflösung von Illitpartikeln hin. Die Al- und Fe-Konzentrationen nehmen ab, obwohl durch den Auflösungsprozeß auch $\mathrm{Al}$ und $\mathrm{Fe}$ in die Lösung abgegeben worden sein müßten. Also auch bei diesem Versuch treten Reaktionen auf, die parallel zum Auflösungsprozeß ablaufen. Hier werden es vor allem Ausfällungsprozesse von Al- und Fe-Hydroxiden sein, die die Al- und FeKonzentration kontrollieren.

Die bei den einzelnen Mineralen besprochenen Reaktionsmechanismen, die sich in zeitlicher Abfolge mit steigendem $\mathrm{pH}$ abspielten (Abb. 6-31), könnten auch Modellcharakter haben für Reaktionsabläufe in Bodenprofilen. Die chemische Zusammensetzung einer Porenlösung wird ja durch verschiedene Einflüsse geprägt, u.a. durch Einträge aus der Atmosphäre, den Chemismus der Festkörper und die pH-Werte der Lösungen. Weiterhin können noch zahlreiche Reaktionen wie Sorptionsreaktionen an Mineraloberflächen, Komplexierungen durch organische Substanzen, Fällungs- oder Lösungsreaktionen ablaufen und sich auf den Chemismus von Porenlösungen auswirken (Barnekow 1996, Schlabach 1996). Viele dieser Reaktionen laufen in den Bodenprofilen simultan ab, denn es handelt sich bei Böden ja um polymineralische Festkörper. Die einzelnen Reaktionen lassen sich also schlecht separat beobachten. 
Im folgenden soll versucht werden, Reaktionsabläufe in einem Bodenprofil durch Vergleich der Änderung von Porenlösungszusammensetzungen mit Meßergebnissen aus Laboruntersuchungen $\mathrm{zu}$ interpretieren. Hierzu ist in Abbildung 6-32a die Tiefenverteilung der in abgepreßten Porenlösungen gemessenen Hauptelementkonzentrationen von einem Quarzit-TonFließerden-Profil aus dem Harz (Schlabach 1996) dargestellt und Meßergebnissen eines BatchVersuches Porenlösung-I-Illit H-35 (Abb. 6-32b) gegenübergestellt. Das Bodenprofil setzt sich zusammen aus einem $\approx 27 \mathrm{~cm}$ mächtigen Torfhorizont mit darunter folgenden Ton-Fließerden mit Quarzitgesteinsbruchstücken. Der tonige Feinboden besteht aus Quarz, Illit, Kaolinit, Chlorit, Feldspat und Goethit/Lepidokrokit (Böttcher 1992).

(a)

Quarzit-Ton-Fließerden-Profil (b)

Illit H-35 mit Porenlösung-I (Batch) DOC: konstant 39,5 $\mathrm{mgC} / 1$

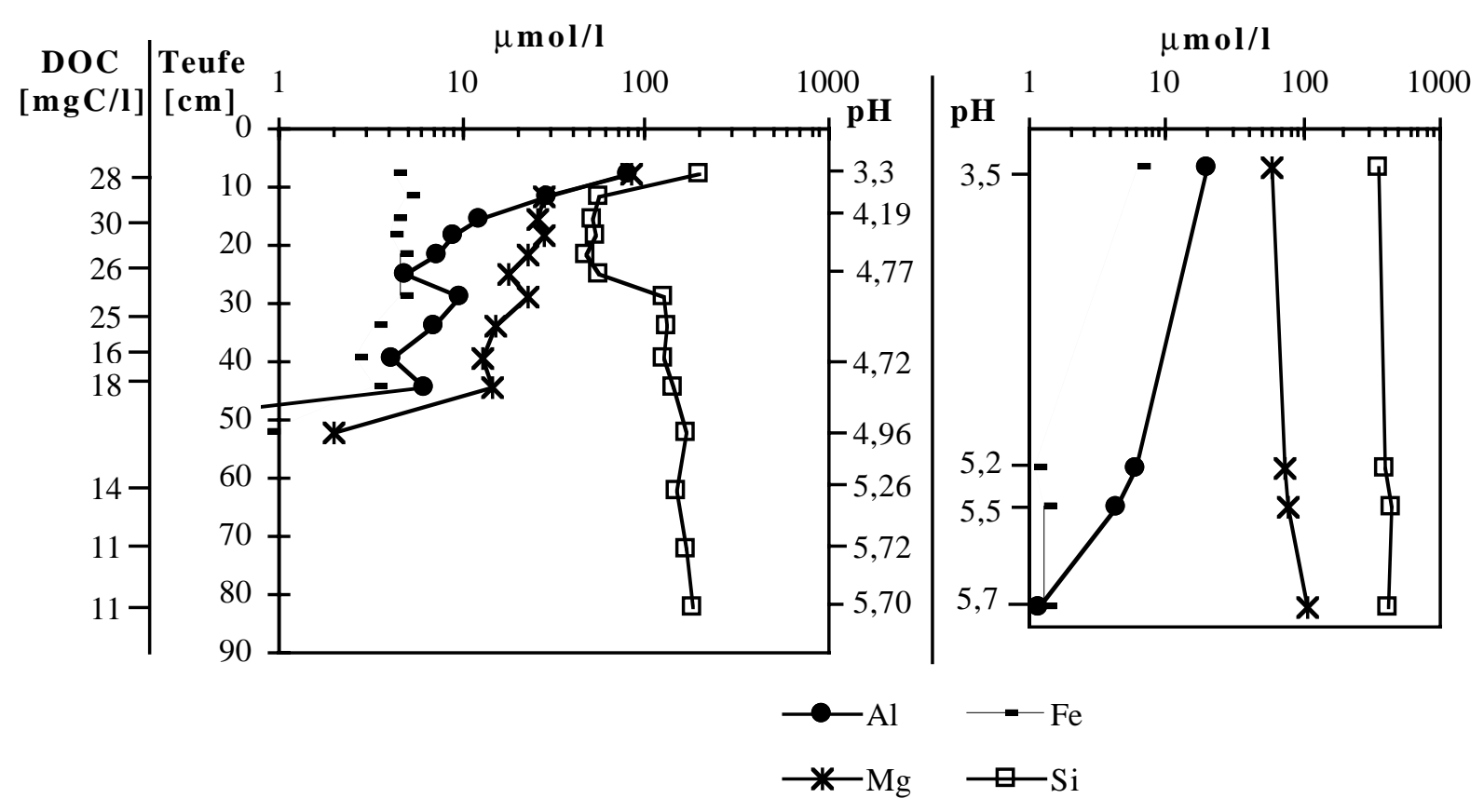

Abb. 6-32: Elementzusammensetzung einer abgepreßten Porenlösungen von einem QuarzitTon-Fließerden-Profil (a; Daten Schlabach 1996) und in einem Batch-Experiment Porenlösung-I-Illit H-35 (b).

Die Porenlösungen des Bodenprofils (Abb. 6-32a) zeichnen sich durch starke Änderungen des pH-Wertes der Lösungen aus. Die Werte steigen von $\approx 3,3$ in den obersten Horizonten auf $\approx 5,7$ in $70-80 \mathrm{~cm}$ Teufe an. Der hohe Anteil organischer Substanz in den oberen $27 \mathrm{~cm}$ des Profils ist sicher verantwortlich für die hohen Al-Gehalte in den Porenlösungen dieses Profilabschnitts. Eine starke Abnahme der Al-, Mg- und Fe-Konzentrationen ist dann bei einem $\mathrm{pH}$-Wert von $\approx 4,7$ in 50 - $60 \mathrm{~cm}$ Teufe zu beobachten, obwohl die entsprechenden Gehalte im Festkörper nahezu konstant bleiben (Barnekow 1996). Die Abnahme der DOC-Werte bei 
gleichzeitig steigendem $\mathrm{pH}$ deuten auf zunehmenden Einfluß von Adsorptions-/Ionenaustauschprozessen und Ausfällungsreaktionen hin.

In Abbildung 6-32b sind die Versuchsergebnisse eines Batch-Versuches von Illit H-35 mit der Porenlösung-I dargestellt. Bei diesem Versuch war es zu einer pH-Drift von anfangs 3,5 auf 5,7 nach 188 Tagen gekommen. Abweichend von der bisherigen Darstellungsweise wurden hier die Konzentrationsänderungen gegen den $\mathrm{pH}-$ Wert der Lösung aufgetragen und zwar gekoppelt an die pH-Verteilung im Bodenprofil. Die leichte Erhöhung der Si- und MgKonzentrationen in der Lösung im Verlauf des Versuches deutet auf Auflösung vor allem der illitischen Hauptkomponente in Probe H-35 hin. Daß die Al- und Fe-Konzentrationen aber nicht ebenfalls ansteigen sondern stark abnehmen, hängt mit dem steigenden pH-Wert zusammen. Die Löslichkeit der Al- und Fe-Hydroxide wird überschritten, und es kommt zur Ausfällung der zunächst wohl noch meist amorphen Phasen.

Im Bodenprofil kontrollieren sicher auch Ausfällungen von Al- und Fe-Hydroxiden im $\mathrm{pH}$ Bereich $\geq 4,7$ die Al- und Fe-Konzentrationen in der Porenlösung. Der im Vergleich mit dem Batch-Experiment geringere DOC-Gehalt in der Porenlösung wird verantwortlich dafür sein, daß im Bodenprofil die Fällungsreaktionen schon bei $\mathrm{pH} \approx 4,7$ einsetzen, bei dem BatchExperiment aber erst bei Werten $\geq 5,2$. Die rasche Abnahme der Mg-Konzentration in der Porenlösung läßt sich vergleichen mit dem Verhalten des Mg bei dem Versuch MontmorillonitPorenlösung-I (Abb. 6-31b), bei dem Adsorptions-/Ionenaustauscheffekte als Erklärung herangezogen wurden.

In einem zweiten Beispiel soll demonstriert werden, daß bei Laborexperimenten Lösungszusammensetzungen erzeugt werden können, die natürlichen Porenlösungen in etwa entsprechen. In Abbildung 6-33 sind Versuchsergebnisse eines Batch-Experimentes mit Illit H35 und $\mathrm{C}_{6} \mathrm{H}_{8} \mathrm{O}_{7}$ der chemischen Zusammensetzung einer Porenlösung in $15 \mathrm{~cm}$ Tiefe von einem Bodenprofil unter Fichte im Solling (Schlabach 1996) gegenübergestellt. Abgesehen von den Fe-Gehalten liegen die Konzentrationen der anderen Elemente nach einer Versuchszeit von 188 Tagen in gleicher Größenordnung wie in der Porenlösung. Die hohen Fe-Gehalte in der Versuchslösung stammen aus der Auflösung der Fe-Hydroxidüberzüge, die sich bei der Probe H-35 auf vielen Partikeln befinden. Die mineralogische Zusammensetzung der Illitprobe H-35 entspricht etwa der Zusammensetzung des Bodenprofils in der betrachteten Tiefe. 


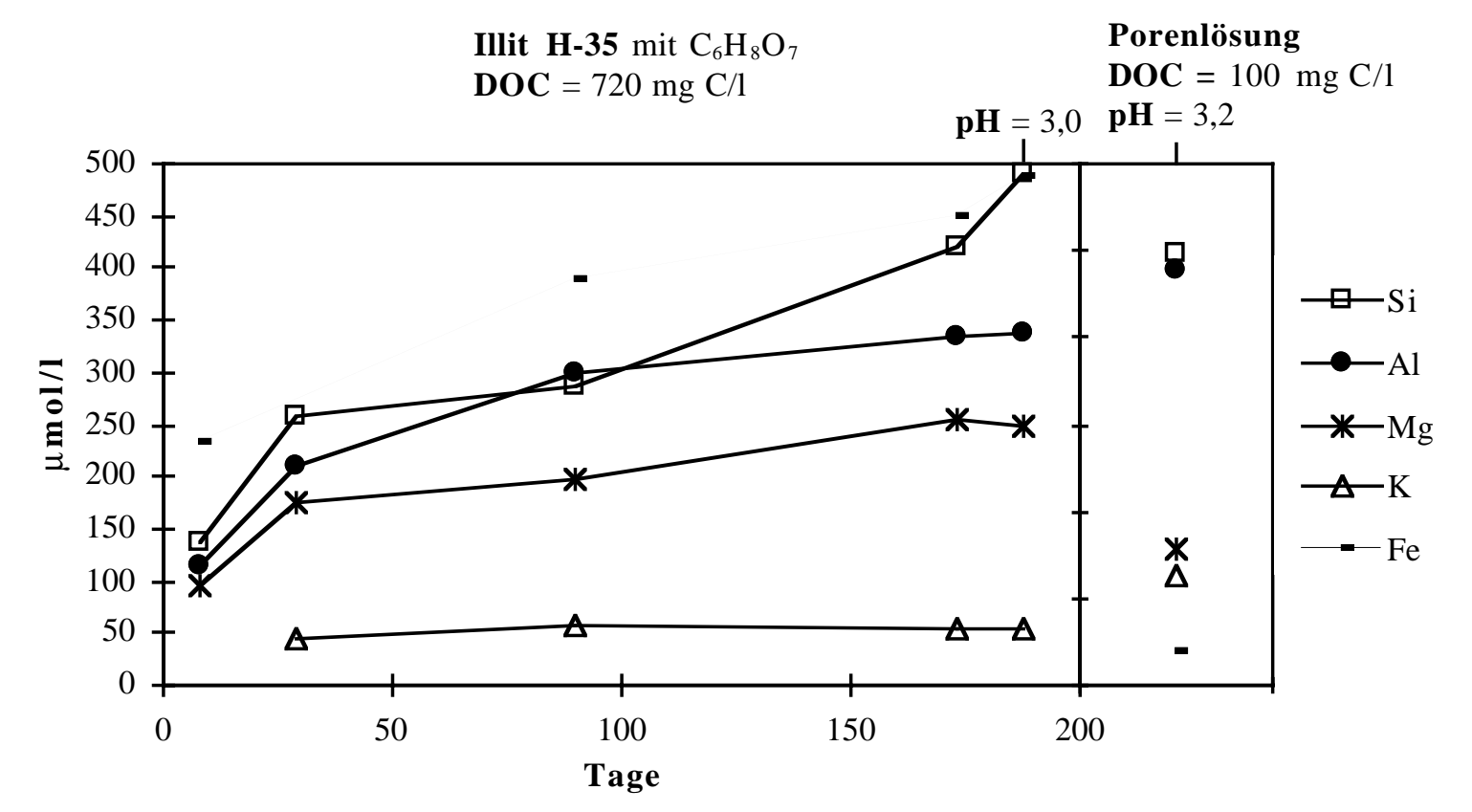

Abb. 6-33: Auflösung von Illit $\mathrm{H}$-35 in $\mathrm{C}_{6} \mathrm{H}_{8} \mathrm{O}_{7}$-saurer Lösung im Batch-Experiment und Elementzusammensetzung einer Porenlösung unter Fichte im Solling bei pH 3,2 in $15 \mathrm{~cm}$ Profiltiefe (vgl. Versuch 25 und Tab. A-2). 


\section{Zusammenfassung}

Die Auflösung der untersuchten Schichtsilikate in sauren Lösungen ist gekennzeichnet durch abnehmende Lösungsgeschwindigkeiten mit steigendem pH-Wert. Die Konzentrationen der in Lösung gegangenen Ionen werden durch verschiedene Prozesse beeinflußt.

Bei den protonenkontrollierten Prozessen können, je nach $\mathrm{pH}-$ Bereich, verschiedene Phänomene beobachtet werden. So liegt bei pH-Werten von 1 - 2 überwiegend kongruente Auflösung der untersuchten Minerale vor, wenn man das Si/Al-Molverhältnis in den Lösungen betrachtet. Im pH-Bereich > 2 und vor allem > 3 laufen simultan mit der Auflösung der Minerale Adsorptionsprozesse und zum Teil auch Ionenaustauschprozesse ab. Besonders deutlich wirken sich diese auf die Al- und Fe-, aber auch auf die Mg-Konzentrationen in den Lösungen aus. Bei weiter steigenden pH-Werten um 4 - 5 ist die Ausfällung von Al- und FeOxidhydroxiden möglich. Deutliche Hinweise für die Bildung solcher Phasen finden sich in einzelnen Experimenten mit den beiden Illiten (Abb. 6-22). Die Bildung von Al-HydroxoSulfaten konnte nicht nachgewiesen werden, obwohl die Ausfällung von Jurbanit aufgrund der Löslichkeitsdaten in einzelnen Experimenten möglich wäre.

Auf zwei Prozesse muß noch hingewiesen werden. Bei den Experimenten mit Montmorillonit beobachtete man bei niedrigen $\mathrm{pH}-$ Werten einen sehr raschen Austausch von Na-Ionen aus dem Zwischenschichtbereich gegen $\mathrm{H}^{+}$-Ionen aus der Lösung (Abb. 6-12, 6-13). Diese $\mathrm{H}^{+}$-Ionen sind nur außersphärisch gebunden und können leicht gegen andere Ionen ausgetauscht werden. Es stellt sich entsprechend der Lösungszusammensetzung ein Gleichgewicht ein. Für die Experimente im pH-Bereich 1 - 2 konnte aus der Menge der ausgetauschten $\mathrm{Na}^{+}$-Ionen die Ionenaustauschkapazität des Montmorillonites abgeschätzt werden. Diese Ionenaustauschkapazität konnte in Beziehung zu den adsorbierten bzw. durch Ionenaustausch gebundenen AlIonen gesetzt werden (Tab. 6-11, 6-12, 6-13).

Liegt für die Zwischenschichtkationen eine innersphärische Bindung vor wie es für das K beim Serizit und den Illiten der Fall ist, so kann das K bei Auflösungsversuchen zwar bevorzugt in Lösung gehen (Tab. 6-17), wird aber nicht schnell und komplett ausgetauscht. Ein solches bevorzugtes Herauslösen von Ionen aus dem Gitter beobachtet man unter bestimmten Bedingungen auch bei einigen Kationen, die auf Oktaederpositionen sitzen. Dies ist vor allem dann der Fall, wenn die Schichtstruktur durch relativ große Ionen, wie Mg und Fe, verzerrt und dadurch destabilisiert wird. Beobachtet werden kann dies insbesondere in den Experimenten mit Montmorillonit (Tab. 6-10), Serizit und den beiden Illiten (Tab. 6-17).

Die Auflösung der Minerale in Anwesenheit von Liganden $\left(\mathrm{C}_{6} \mathrm{H}_{8} \mathrm{O}_{7}, \mathrm{DOC}, \mathrm{HClO}_{4}\right)$ zeichnet sich durch stöchiometrische Lösungszusammensetzungen auch bei pH-Werten > 3 aus. Liganden sind also in der Lage, vor allem Al- und Fe-Ionen in der Lösung zu komplexieren. Adsorptions- und Ionenaustauschprozesse sind weitgehend unterbunden. 
Werden die Auflösungsversuche in Gegenwart von Elektrolytzusatz durchgeführt, so werden auch in diesem Fall die Adsorptions- und Ionenaustauschprozesse stark eingeschränkt. Die in höheren Konzentrationen vorliegenden Ionen des Elektrolytzusatzes besetzen die Positionen der Oberflächenladungen der Minerale. Bei der Gleichgewichtseinstellung zwischen den Ionen in der Lösung und den an der Mineraloberfläche fixierten Ionen spielen die niedrigen Konzentrationen der beim Auflösungsprozeß in Lösung gehenden Ionen kaum eine Rolle.

Neben den pH-Werten scheinen auch die Säure-Anionen einen Einfluß auf das Auflösungsverhalten der Minerale zu haben. So konnte beim Kaolinit in Anwesenheit von $\mathrm{HNO}_{3}$ und $\mathrm{HCl}$ auch bei $\mathrm{pH}-$ Werten um 1 nichtstöchiometrische Auflösung mit $\mathrm{R}_{\mathrm{Si}}>\mathrm{R}_{\mathrm{Al}}$ beobachtet werden.

Der Abbau der in dieser Arbeit betrachteten Minerale erfolgt überwiegend über die Mineralkanten, was dem in Kapitel 2 theoretisch beschriebenen Prozeß entspricht. Sichtbares Merkmal sind die zunehmenden Kantenrundungen. Für den Serizit konnte außerdem die Bildung von Lösungsspuren und Ätzgruben beobachtet werden (Abb. 6-18), die vermutlich auf Defektstellen in der Mineralstruktur zurückzuführen sind. Trotz dieses abweichenden Befundes in der Oberflächenmorphologie konnte für den Serizit bei niedrigen $\mathrm{pH}$-Werten stöchiometrische Auflösung (bezogen auf das Si/Al-Verhältnis in der Lösung) beobachtet werden.

Möglicherweise hat, wie von Sutheimer et al. (1999) vermutet, die Struktur der Minerale, d.h. das Verhältnis von Kanten- zu Basalflächen, den größten Einfluß auf die Auflösung der Minerale. Dieses Verhältnis ist abhängig von der Korngröße und der Kristallinität der Partikel und kann, wie bereits in Kapitel 2 erwähnt, stark variieren. Bei Kaoliniten liegt der Anteil der Seitenflächen an der Gesamtoberfläche beispielsweise zwischen 20 und 47\% (Nagy 1995).

Die untersuchten Minerale lösen sich also in sauren Lösungen überwiegend kongruent auf. Nichtstöchiometrische Lösungszusammensetzungen sind auf simultan mit der Auflösung ablaufende Prozesse zurückzuführen. Den geringsten Einfluß haben derartige Prozesse auf das gelöste Si, womit die Si-Konzentrationen die Mineralauflösung am besten widerspiegeln.

Für die Zusammensetzung einer Bodenlösung ist nicht allein die Geschwindigkeit der Mineralauflösung von Bedeutung. Wichtige Faktoren sind die auftretenden sekundären Prozesse, die zu einem großen Teil von der Zusammensetzung der Lösungen abhängen. So können verschiedene Säure-Anionen, die über atmosphärische Depositionen in das System Boden eingetragen werden, unterschiedlich auf die Mineralverwitterung wirken. Besondere Bedeutung kommt den stark komplexierend wirkenden organischen Liganden aus der Biomasse zu, die großen Einfluß auf die Al- und Fe-Konzentrationen in den Lösungen haben. Auch sind die Auflösungsgeschwindigkeiten in ligandengesteuerten Systemen größer als in rein protonengesteuerten. Interessant wären daher Experimente mit natürlich vorkommenden DOC- 
Zusammensetzungen. Die in dieser Arbeit in einzelnen Experimenten verwendeten abgepreßten Porenlösungen bzw. der Vergleich von experimentell erzeugten Lösungszusammensetzungen mit Porenlösungszusammensetzungen stellen einen ersten Schritt in diese Richtung dar. Die schwierige chemische Charakterisierung von DOC und die bereits hohen Ionenkonzentrationen in den Porenlösungen erschweren die Interpretation der Ergebnisse. Des weiteren können die natürlich vorkommenden DOC-Zusammensetzungen auf kleinstem Raum sehr variabel sein. Für zukünftige Untersuchungen ist daher die Verwendung einzelner organischer Verbindungen mit definierten funktionellen Gruppen als Modellsubstanzen für DOC eine sinnvolle Alternative. Generell kann man sagen, daß experimentelle Untersuchungen geeignet sind, den maximalen Elementbeitrag in Bodenlösungen aus der Verwitterung der Minerale zu betrachten. Parameter, wie unterschiedliche $\mathrm{pH}$-Werte, Temperaturen und Lösungszusammensetzungen können leicht variiert werden. Aus den experimentell gewonnenen Auflösungsgeschwindigkeiten können Rückschlüsse auf Reaktionsmechanismen von Verwitterungsabläufen gezogen werden. Die Informationen eignen sich zur Modellierung von Verwitterungsprozessen. 


\section{Literatur}

Amacher M C (1991) Methods of Obtaining and Analyzing Kinetic Data.- In: Rates of Soil Chemical Processes (eds. D L Sparks, D L Suarez) Soil Science Society of America, Special Publication No 27, Madison, U.S.A., pp. 19-59.

Anbeek C (1993) The effect of natural weathering on dissolution rates. Geochimica et Cosmochimica Acta 57: 4963-4975.

Arias M, Barral M T, Diaz-Fierros F (1995) Effects of iron and aluminium oxides on the colloidal and surface properties of kaolin.- Clays and Clay Minerals 43: 406-416.

Ball J W, Nordstrom D K, Jenne E A (1980) Additional and reversed thermochemical data and computer code for WATEQ2- a computerized chemical model for trace and major element speciation and mineral equilibria of natural waters.- U.S. Geological Survey WaterResources Investigation 78-116: 101-109.

Barnekow P (1996) Der Einfluß der Silikatverwitterung auf den Chemismus abgepreßter Porenlösungen von versauerten Bodenprofilen (Harz, Solling).- Diplomarbeit am Geochemischen Istitut der Universität Göttingen, pp. 94.

Bloom P R, Nater E A (1991) Kinetics of dissolution of oxide and primary silicate minerals.In: Rates of Soil Chemical Processes (eds. D L Sparks, D L Suarez) Soil Science Society of America, Special Publication No 27, Madison, U.S.A., pp. 151-189.

Böttcher G (1992) Wechselwirkungen zwischen Festphasen und Lösungen (z.B. unter Bildung basischer Aluminiumsulfate) in 5 Bodenprofilen über verschiedenartigen Gesteinen in der Sösemulde (Oberharz).- Berichte des Forschungszentrums Waldökosysteme Göttingen A93: pp. 84.

Böttcher G, Brumsack H-J, Heinrichs H, Pohlmann M (1997) A new high-pressure squeezing technique for pore fluid extraction from terrestrial soils.- Water, Air and Soil Pollution 94: 289-296.

Boss C B, Fredeen K J (1997) Concepts, Instrumentation, and Techniques in Inductively Coupled Plasma Optical Emission Spectrometry. The Perkin Elmer Corporation, Norwalk, CT, pp 101.

Breemen N van, Driscoll C T, Mulder J (1984) Acidic deposition and internal proton sources in acidification of soils and water.- Nature 307: 599-604.

Caroll S A, Walther J V (1990) Kaolinite dissolution at $25^{\circ}, 60^{\circ}$ and $80^{\circ} \mathrm{C}$.- American Journal of Science 290: 797-810.

Carroll-Webb S A, Walther J V (1988) A surface complex reaction model for the $\mathrm{pH}$ dependence of corundum and kaolinite dissolution rates.- Geochimica et Cosmochimica Acta 52: 2609-2623.

Casey W H, Banfield J F, Westrich H R, McLaughlin L (1993) What do dissolution experiments tell us about natural weathering?- Chemical Geology 105: 1-15.

Deutschmann G (1994) Zustand und Entwicklung der Versauerung des Bodens und des oberflächennahen Buntsandsteinuntergrundes eines Waldökosystems im Solling.- Berichte des Forschungszentrums Waldökosysteme/Waldsterben Göttingen 16: pp. 118.

Dietzel M (1998) Gelöste polymere und monomere Kieselsäuren und die Wechselwirkungen mit Gibbsit und Fe-O-OH-Festphasen.- Habilitationsschrift, Fakultät für Geowissenschaften, Georg-August-Universität Göttingen, pp. 240. 
Drever J I (1973) The Preperation of Oriented Clay Mineral Specimens for X-ray Diffraction Analysis by Filter-Membrane Peel Technique.- American Mineralogist 58: 553-554.

Drever J I (1997) Catchment mass balance. In: Geochemical Processes, Weathering and Groundwater Recharge in Catchments (eds. O M Saether, P de Caritat) A.A. Balkema, Rotterdam, Brookfield, pp. 241-261.

Drever J I, Clow D W (1995) Weathering rates in catchments. In: Chemical weathering rates of silicate minerals (eds. A F White \& S L Brantley) Reviews in Mineralogy 31: 463-483.

Feigenbaum S, Shainberg I (1975) Dissolution of illite - a possible mechanism of potassium release.- Soil Science Society of America Proceedings 39: 985-990

Flehmig W, Kurze R (1973) Die quantitative infrarotspektroskopische Phasenanalyse von Mineralmengen.- Neues Jahrbuch der Mineralogie, Abhandlungen 119: 101-112.

Frei M, Bielert U, Heinrichs H (2000) Effects of pH, alkalinity and bedrock chemistry on metal concentrations of springs in an acidified catchment (Ecker Dam, Harz Mountains, FRG).Chemical Geology 170: 221-242.

Furrer G, Zysset M, Schindler P W (1993) Weathering kinetics of montmorillonite: Investigations in batch and mixed-flow reactors. In: Geochemistry of Clay-Pore Fluid Interactions (eds. D A C Manning, P L Hall \& C R Hughes) Mineralogical Society Series 4, Chapman \& Hall, London, pp. 243-262.

Galan E, Carretero M I, Fernandez-Caliani J C (1999) Effects of acid mine drainage on clay minerals suspended in the Tinto River (Rio Tinto, Spain). An experimental approach.- Clay Minerals 34: 99-108.

Ganor J, Mogollón J L, Lasaga A C (1995) The effect of pH on kaolinite dissolution rates and on activation energy.- Geochimica et Cosmochimica Acta 59: 1037-1052.

Hartmann G (1994) Late-medieval glass manufacture in the Eichsfeld Region (Thuringia, Germany).- Chemie der Erde 54: 103-128.

Heinrichs H, Herrmann A G (1990) Praktikum der Analytischen Geochemie.- 1. Auflage, Springer, Berlin, pp 669.

Heinrichs H, Wachtendorf B, Wedepohl K H, Rössner B, Schwedt G (1986) Hydrogeochemie der Quellen und kleineren Zuflüsse der Sösetalsperre (Harz).- Neues Jahrbuch für Mineralogie, Abhandlungen 156:23-62.

Heydemann A (1966) Über die chemische Verwitterung von Tonmineralen (Experimentelle Untersuchungen).- Geochimica et Cosmochimica Acta 30: 995-1035.

Hochella M F, Banfield J F (1995) Chemical weathering of silicates in nature: a microscopic perspective with theoretical considerations. In: Chemical weathering rates of silicate minerals (eds. A F White \& S L Brantley) Reviews in Mineralogy 31: 353-406.

Hower J, Mowatt T C (1966) The mineralogy of illites and mixed-layer illite/montmorillonites.American Mineralogist 51: 825-854.

Huang W H, Keller W D (1971) Dissolution of clay minerals in dilute organic acids at room temperature.- American Mineralogist 56: 1082-1095.

Huertas F J, Chou L, Wollast R (1998) Mechanism of kaolinite dissolution at room temperature and pressure: Part 1. Surface speciation.- Geochimica et Cosmochimica Acta 62: 417-431. 
Jasmund K, Lagaly G [Hrsg.] (1993) Tonminerale und Tone. Struktur, Eigenschaften, Anwendungen und Einsatz in Industrie und Umwelt.- Steinkopff, Darmstadt, pp. 490.

Kalinowski B E, Schweda P (1996) Kinetics of muscovite, phlogopite, and biotite dissolution and alteration at $\mathrm{pH}$ 1-4, room temperature.- Geochimica et Cosmochimica Acta 60: 367385 .

Knauss K G, Wolery T J (1989) Muscovite dissolution kinetics as a function of $\mathrm{pH}$ and time at 70 C.- Geochimica et Cosmochimica Acta 53: 1493-1501.

Kuntze H, Herms U (1986) Bedeutung geogener und pedogener Faktoren für die weitere Belastung der Böden mit Schwermetallen.- Naturwissenschaften 73: 195-204.

Lasaga A C (1981) Rate laws of chemical reactions. In: Kinetics of geochemical processes (eds. A C Lasaga \& R J Kirkpatrick) Reviews in Mineralogy 8: pp. 1-68.

Lindsay W L (1979) Chemical equilibria in soils.- 1. Auflage, Wiley \& Sons, Inc., New York, pp. 449.

Malessa V (1993) Depositionsbedingte Tiefengradienten der Bodenversauerung in der Sösemulde (Westharz).- Berichte des Forschungszentrums Waldökosysteme Göttingen A98: pp. 233.

Malessa V, Andreae H (1990) Lage und Ausdehnung der Versauerungsfront in der Verwitterungsdecke von Diabas, Grauwacke/Tonschiefer und Quarzit an Beispielen aus der Sösemulde.- Berichte des Forschungszentrums Waldökosysteme Göttingen B19: pp. 52.

Matschullat J, Heinrichs H, Schneider J, Siewers U (1990) Schwermetalle und Gewässerversauerung - Untersuchungen zum Verhalten von Schadstoffen in bewaldeten Ökosystemen im Harz.- Zeitschrift der deutschen geologischen Gesellschaft 141: 139-150.

Metz V, Ganor J (1998): Auflösungsgeschwindigkeit von Kaolinit in Abhängigkeit vom Suspensionsgrad.- Berichte der Deutschen Mineralogischen Gesellschaft, Beihefte zum European Journal of Mineralogy 10: 192.

Metz V, Ganor J (1999) Congruent dissolution of smectite in a flow-through system. In: Mineral/Water Interactions Close to Equilibrium: Workshop of the Forschungszentrum Karlsruhe held at Speyer 25th and 26th of March 1999 (eds. W Schüßler \& A Bauer) Wissenschaftliche Berichte FZKA 6291, Forschungszentrum Karlsruhe, Technik und Umwelt, pp. 87-91.

Morel F M M, Hering J G (1993) Principles and applications of aquatic chemistry.- John Wiley \& Sons, Inc., New York, pp. 588.

Nagy K L (1995) Dissolution and precipitation kinetics of sheet silicates. In: Chemical weathering rates of silicate minerals (eds. A F White \& S L Brantley) Reviews in Mineralogy 31: 173-233.

Nagy K L, Sturchio N C (1997) Growth kinetics of gibbsite in kaolinite powders.- 11th International Clay Conference Ottawa, Program Abstracts: A54.

Nickel E (1973) Experimental dissolution of light and heavy minerals in comparison with weathering and intrastratal solution.- Contributions to Sedimentology 1: 1-68.

Nordstrom D K (1982) The effect of sulfate on aluminum concentrations in natural waters: some stability relations in the system $\mathrm{Al}_{2} \mathrm{O}_{3}-\mathrm{SO}_{3}-\mathrm{H}_{2} \mathrm{O}$ at $298 \mathrm{~K}$.- Geochimica et Comochimica Acta 46: 681-692. 
Pleßow A, Bielert U, Heinrichs H, Steiner I (1997) Problematik der Grundwasserversauerung und das Lösungsverhalten von Spurenstoffen. In: Geochemie und Umwelt. Relevante Prozesse in Atmo-, Pedo- und Hydrosphäre (Hrsg. J Matschullat, H J Tobschall \& H-J Voigt) Springer, pp. 395-408.

Raupach M (1960) Aluminium Ions in Aluminium Hydroxide, Phosphate and Soil-Water Systems.- Nature 188: 1049-1050.

Reuss J O, Cosby B J, Wright RF (1987) Chemical processes governing soil and water acidification.- Nature 329: 27-32.

Scheffer F, Schachtschabel P (1992) Lehrbuch der Bodenkunde.- 13. durchgesehene Auflage, Enke, Stuttgart, pp. 491.

Scheffer F, Schachtschabel P (1998) Lehrbuch der Bodenkunde.- 14. neu bearbeitete und erweiterte Auflage, Enke, Stuttgart, pp. 494.

Schindler D W (1988) Effects of acid rain on freshwater ecosystems.- Science 239: 149-157.

Schlabach S (1996) Konzentrationsänderungen natürlicher und anthropogener Inhaltsstoffe in abgepreßten Porenlösungen mit dem $\mathrm{pH}-$ Wert und der Tiefe auf versauerten Waldstandorten (Harz, Solling).- Diplomarbeit am Geochemischen Istitut der Universität Göttingen, pp. 99.

Schnoor J L (1990) Kinetics of chemical weathering: A comparison of laboratory and field weathering rates. In: Aquatic Chemical Kinetics (ed. W Stumm) Wiley-Interscience, New York, pp. 475-504.

Schroth B K, Sposito G (1997) Surface charge properties of kaolinite.- Clays and Clay Minerals 45: 85-91.

Sposito G (1998) Bodenchemie.- Übersetzt von J Niemeyer und Andrea Eifert, Enke, Stuttgart, pp. 230.

Stone A T (1997) Reactions of extracellular organic ligands with dissolved metal ions and mineral surfaces. In: Geomicrobiology: interactions between microbes and minerals (eds. J F Banfield \& K H Nealson) Reviews in Mineralogy 35: pp. 309-344.

Stumm W (1992) Chemistry of the solid-water interface. Processes at the mineral-water and particle-water interface in natural systems.- Wiley \& Sons, Inc., New York, pp. 428.

Stumm W, Morgan J J (1996) Aquatic chemistry. Chemical equilibria and rates in natural waters.- 3. Edition, Wiley \& Sons, Inc., New York, pp. 1022.

Stumm W, Wehrli B, Wieland E (1987) Surface complexation and its impact on geochemical kinetics.- Croatica Chemica Acta 60: 265-276.

Sutheimer S H, Maurice P A, Zhou Q (1999) Dissolution of well and poorly crystallized kaolinites: Al speciation and effects of surface characteristics.- American Mineralogist 84: 620-628.

Sverdrup H U, Warfvinge P (1988) Weathering of primary silicate minerals in the natural soil environment in relation to a chemical weathering model.- Water, Air and Soil Pollution 38: 387-408.

Swoboda-Colberg N G, Drever J I (1993) Mineral dissolution rates in plot-scale field and laboratory experiments.- Chemical Geology 105: 51-69.

Velbel M A (1993) Constancy of silicate-mineral weathering-rate ratios between natural and experimental weathering: implications for hydrologic control of differences in absolute rates.- Chemical Geology 105: 89-99. 
Ward D B, Brady P V (1998) Effect of Al and organic acids on the surface chemistry of kaolinite.- Clays and Clay Minerals 46: 453-465.

Wieland E, Stumm W (1992) Dissolution kinetics of kaolinite in acidic aqueous solutions at $25^{\circ} \mathrm{C}$.- Geochimica et Cosmochimica Acta 56: 3339-3355.

Wieland E, Wehrli B, Stumm W (1988) The coordination chemistry of weathering: III. A generalization on the dissolution rates of minerals.- Geochimica et Cosmochimica Acta 52:1969-1981.

Wilson M J [Hrsg.] (1987) A handbook of determinative methods in clay mineralogy.- 1. Edition, Blackie, London, pp. 308.

Zysset M, Schindler P W (1996) The proton promoted dissolution kinetics of Kmontmorillonite.- Geochimica et Cosmochimica Acta 60: 921-931. 


\section{Anhang}

\subsection{Abbildungs- und Tabellenverzeichnis des Anhangs}

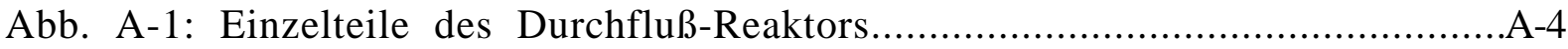

Abb. A-2: Beschreibung der Reaktorteile für die Durchfluß-Experimente................... 5

Abb. A-3: Schematische Darstellung der Sedimentpresse nach Böttcher et al. (1997). .......A-6

Tab. A-1: Charakterisierung der in den Experimenten benutzten abgepreßten Poren-Lösung-I von einem Bodenprofil unter Buche aus dem Solling vom Tiefenbereich 0 -15 cm..................................................................

Tab. A-2: Charakterisierung der als Vergleich dienenden abgepreßten Poren-Lösung von einem Tonstein-Bodenprofil unter Fichte aus dem Solling.

Tab. A-3: Charakterisierung der als Vergleich dienenden abgepreßten Poren-Lösung von einem Quarzit-Ton-Fließerden-Bodenprofil aus dem Harz ....................

Tab. A-4: Chemische Charakterisierung der Mineralproben

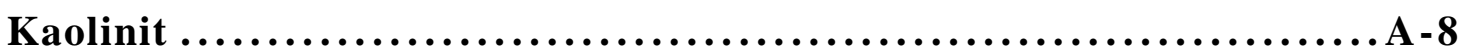

Tab. A-5: Batch-Experimente mit Kaolinit Versuche 1 und 2. .......................... 8

Tab. A-6: Batch-Experimente mit Kaolinit Versuche 3 und 4. .........................

Tab. A-7: Batch-Experimente mit Kaolinit Versuche 5 und 7. ....................... A-10

Tab. A-8: Batch-Experimente mit Kaolinit Versuche 8 und $18 \ldots \ldots \ldots \ldots \ldots \ldots \ldots \ldots \ldots \ldots \ldots \ldots \ldots \ldots$

Tab. A-9: Batch-Experimente mit Kaolinit Versuch 47........................................ A-11

Tab. A-10: Durchfluß-Experimente mit Kaolinit Versuch II......................... A-12

Tab. A-11: Durchfluß-Experimente mit Kaolinit Versuch IV90(1) .................... A-13

Tab. A-12: Durchfluß-Experimente mit Kaolinit Versuch IV90(2). ................... A-14

Tab. A-13: Durchfluß-Experimente mit Kaolinit Versuch IV250(1)................... A-15

Tab. A-14: Durchfluß-Experimente mit Kaolinit Versuch IV250(2).................... A-16

Tab. A-15: Durchfluß-Experimente mit Kaolinit Versuch IV250(4).................... A-17

Tab. A-16: Durchfluß-Experimente mit Kaolinit Versuch V90.......................... A-18

Tab. A-17: Durchfluß-Experimente mit Kaolinit Versuch V250(2)..................... A-19

Tab. A-18: Durchfluß-Experimente mit Kaolinit Versuch VI90....................... A-20

Tab. A-19: Durchfluß-Experimente mit Kaolinit Versuch VI250(1)....................A-21

Tab. A-20: Durchfluß-Experimente mit Kaolinit Versuche VI250(2) und VI250(3).......... A-22

Tab. A-21: Durchfluß-Experimente mit Kaolinit Versuche VI250(4) und X(1)............ A-23

Tab. A-22: Durchfluß-Experimente mit Kaolinit Versuch X(2) ....................... A-24 
Montmorillonit A-25

Tab. A-23: Batch-Experimente mit Montmorillonit Versuche 16 und 17 A-25

Tab. A-24: Batch-Experimente mit Montmorillonit Versuche 26 und 30. A-26

Tab. A-25: Batch-Experimente mit Montmorillonit Versuche 37 und 38 A-27

Tab. A-26: Batch-Experimente mit Montmorillonit Versuche 39 und 45 A-28

Tab. A-27: Batch-Experimente mit Montmorillonit Versuch 49. A-29

Tab. A-28: Durchfluß-Experimente mit Montmorillonit Versuch VIII(1). A-30

Tab. A-29: Durchfluß-Experimente mit Montmorillonit Versuch VIII(2). A-31

Tab. A-30: Durchfluß-Experimente mit Montmorillonit Versuch VIII(3). A-32

Tab. A-31: Durchfluß-Experimente mit Montmorillonit Versuch XIV. A-33

Serizit $. A-34$

Tab. A-32: Batch-Experimente mit Serizit Versuche 21 und 24. A-34

Tab. A-33: Batch-Experimente mit Serizit Versuche 44 und 50. A-35

Tab. A-34: Batch-Experimente mit Serizit Versuche 51 und 52. A-36

Tab. A-35: Batch-Experimente mit Serizit Versuche 53 und 54. A-37

Tab. A-36: Batch-Experimente mit Serizit Versuch 55. A-38

Tab. A-37: Durchfluß-Experimente mit Serizit Versuche IX(1)und IX(2)........ A-39

Tab. A-38: Durchfluß-Experimente mit Serizit Versuch XV. A-40

Illit H-35. A - 41

Tab. A-39: Batch-Experimente mit Illit H-35 Versuche 10 und 11. A-41

Tab. A-40: Batch-Experimente mit Illit H-35 Versuche 25 und 28. A-42

Tab. A-41: Batch-Experimente mit Illit H-35 Versuche 31 und 32. A-43

Tab. A-42: Batch-Experimente mit Illit H-35 Versuche 33 und 46. A-44

Tab. A-43: Durchfluß-Experimente mit Illit H-35 Versuch VII(1). A- -45

Tab. A-44: Durchfluß-Experimente mit Illit H-35 Versuch VII(2). A-46

Tab. A-45: Durchfluß-Experimente mit Illit H-35 Versuch VII(3). A-47

Illit OECD A-48

Tab. A-46: Batch-Experimente mit Illit OECD Versuche 19 und $22 \ldots \ldots \ldots \ldots \ldots \ldots \ldots \ldots$ A-48

Tab. A-47: Batch-Experimente mit Illit OECD Versuche 29 und $34 \ldots \ldots \ldots \ldots \ldots \ldots \ldots . \ldots . \ldots . \ldots$

Tab. A-48: Batch-Experimente mit Illit OECD Versuche 35 und $36 \ldots \ldots \ldots \ldots \ldots \ldots \ldots . . \ldots$ A-50

Tab. A-49: Batch-Experimente mit Illit OECD Versuche 43 und 48..................... A-51

Tab. A-50: Durchfluß-Experimente mit Illit OECD Versuch XII(1) ..................... A-52

Tab. A-51: Durchfluß-Experimente mit Illit OECD Versuch XII(2).................... A-53 
Talk.............................................................A-54

Tab. A-52: Batch-Experimente mit Talk Versuche 20 und 23...................... A-54

Tab. A-53: Durchfluß-Experimente mit Talk Versuch XI........................... A-55

Tab. A-54: Durchfluß-Experimente mit Talk Versuch XIII........................ A-56 


\subsection{Abbildungen}

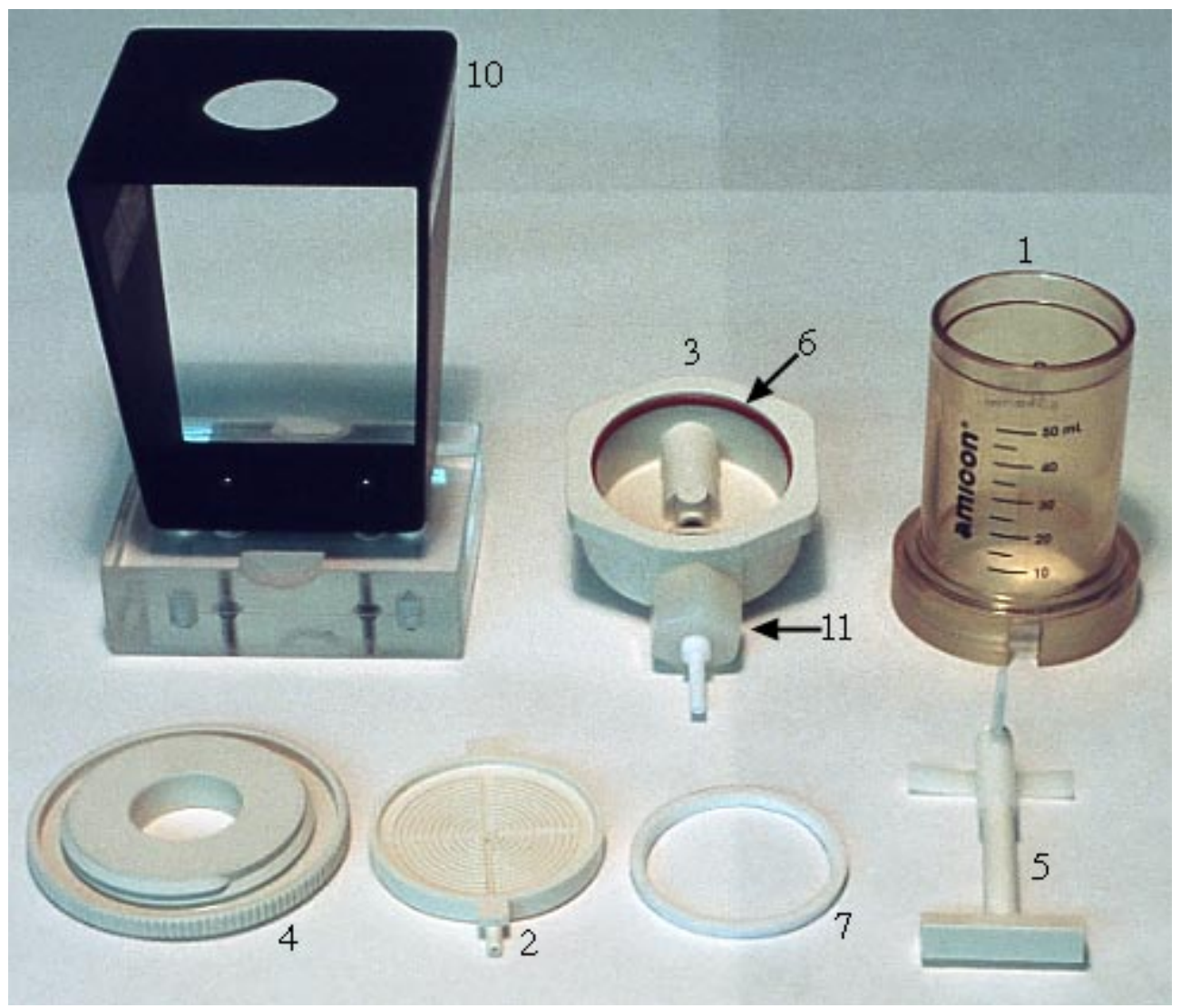

Abb. A-1: Einzelteile des Durchfluß-Reaktors. Beschreibung der Teile s. Abb. A-2 


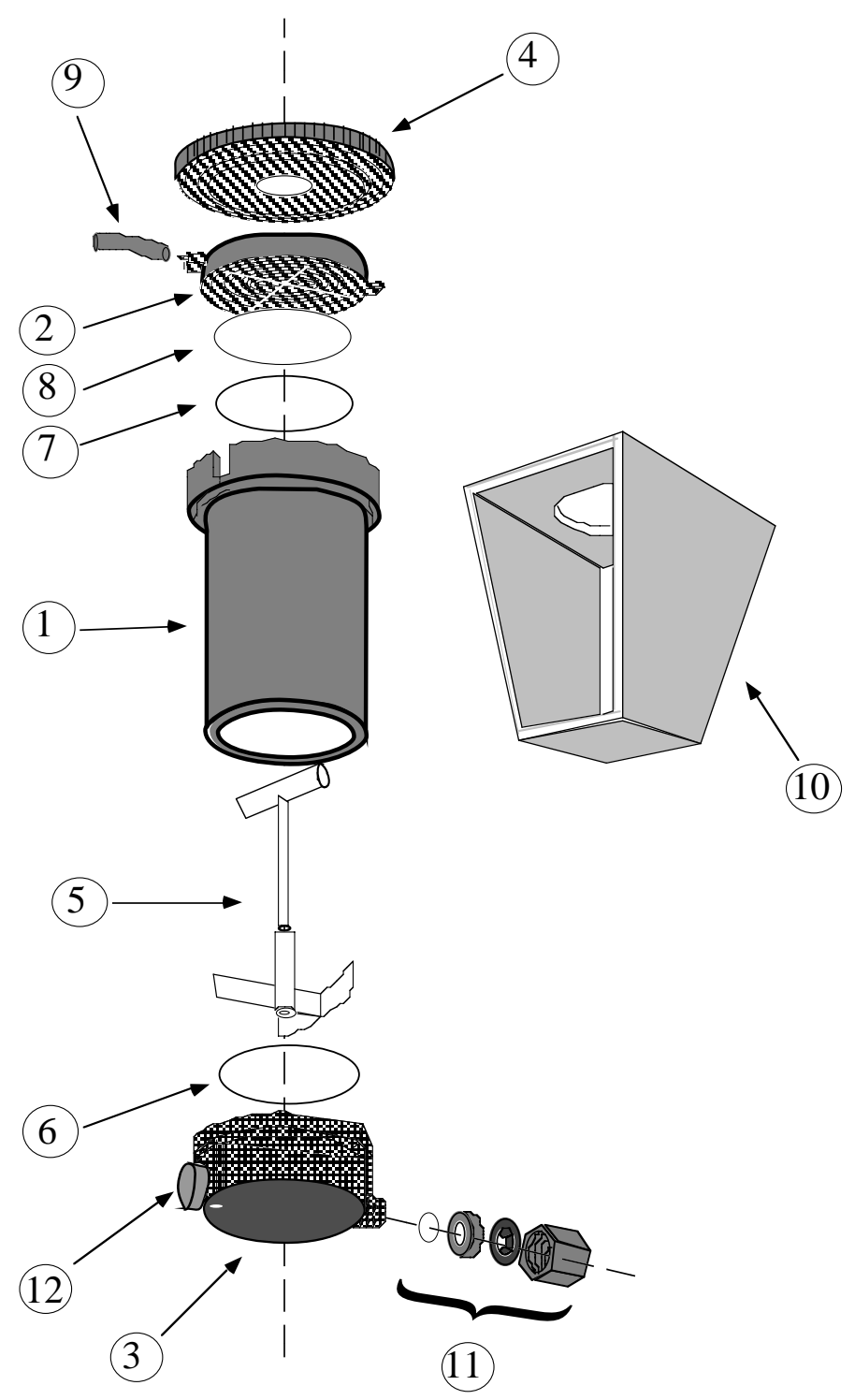

(1) Behälter -> Polysulphon

(2) Membran - Halter -> Polysulphon

(3) Deckel -> Nylon

(4) Basisplatte -> Polysulphon

5 Rührer -> Acetal, Polysulphon

6 O - Ring -> Silikon
(7) O - Ring -> PTFE bzw. Viton ${ }^{\circledR}$

(8) Filtermembran -> PTFE

9 Schlauch -> Tygon $®$

(10) Halter $->$ Aluminium

(11) Schlauchanschluß - Ventil -> Nylon

(12) Überdruck-Ventil

Abb. A-2: Beschreibung der Reaktorteile für die Durchfluß-Experimente. Der unter 6 aufgeführte O-Ring aus Silikon steht nicht in Kontakt mit der Lösung. 


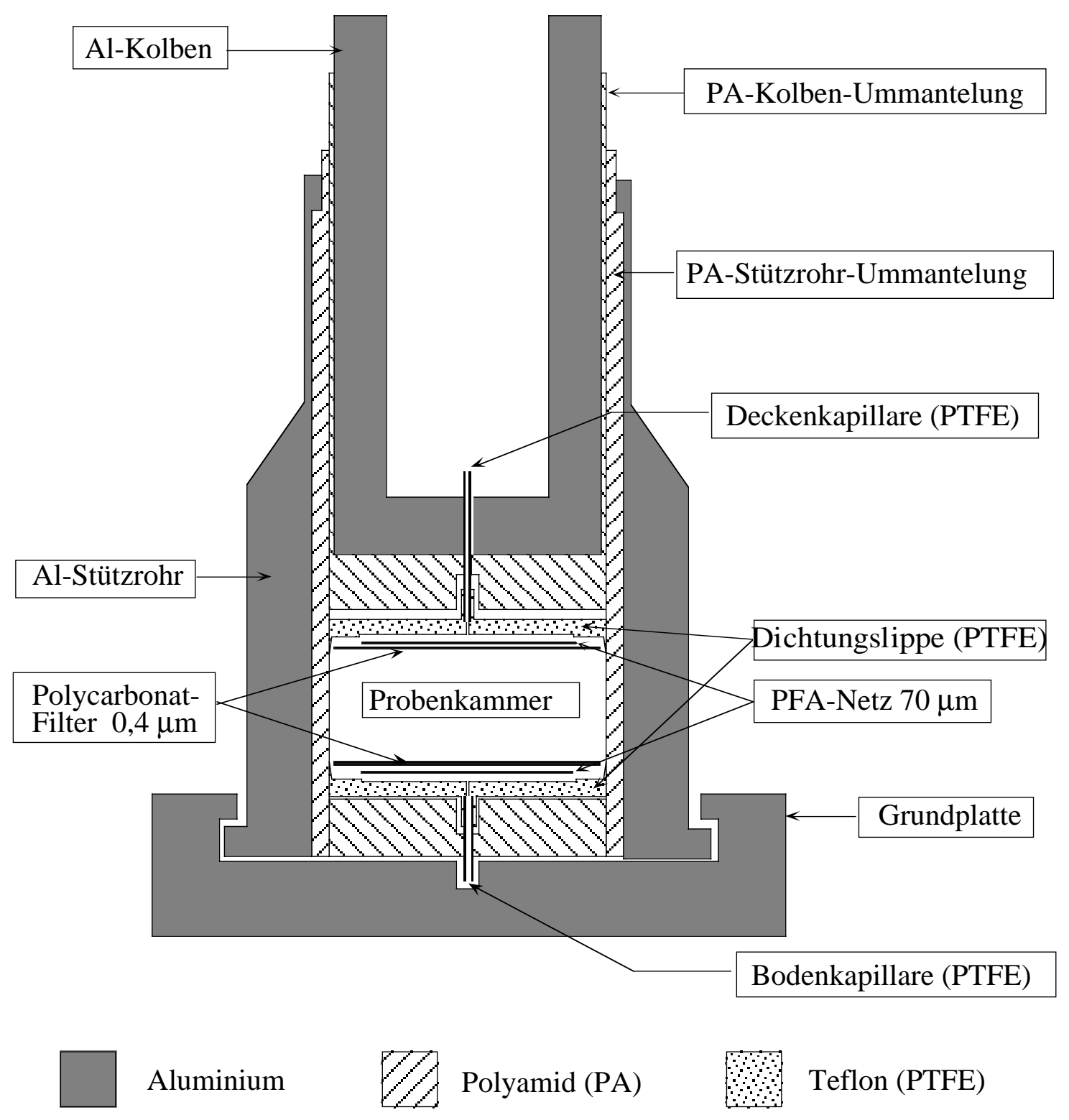

Abb. A-3: Schematische Darstellung der Sedimentpresse nach Böttcher et al. (1997). 


\subsection{Tabellen}

Die für die Bestimmung der Auflösungsraten R benutzten Daten sind bei den DurchflußExperimenten grau hinterlegt bzw. durch die Angabe des Zeitraumes kenntlich gemacht. Bei den Batch-Experimenten wurden meist die Daten des gesamten Zeitraumes benutzt. Bei Abweichungen von dieser Regel ist dies ebenfalls durch Markieren der benutzten Daten hervorgehoben. Die Abkürzung „n.b.” steht für „nicht bestimmt”.

Tab. A-1: Charakterisierung der in den Experimenten benutzten abgepreßten Poren-Lösung-I von einem Bodenprofil unter Buche aus dem Solling vom Tiefenbereich $0-15 \mathrm{~cm}$.

\begin{tabular}{|c|ccccccc|}
\hline & $\mu \mathrm{mol} / \mathrm{l}$ & & & & & & $\mathrm{mg} / \mathrm{l} \mathrm{C}$ \\
\hline $\mathbf{p H}$ & $\mathbf{A ~ l}$ & $\mathbf{F e}$ & $\mathbf{K}$ & $\mathbf{M g}$ & $\mathbf{N a}$ & $\mathbf{S ~ i}$ & $\mathbf{D O C}$ \\
3,5 & 20 & 6,3 & 58 & 59 & 328 & 362 & 39 \\
\hline
\end{tabular}

Tab. A-2: Charakterisierung der als Vergleich dienenden abgepreßten Poren-Lösung von einem Tonstein-Bodenprofil unter Fichte aus dem Solling (Daten aus Schlabach 1996).

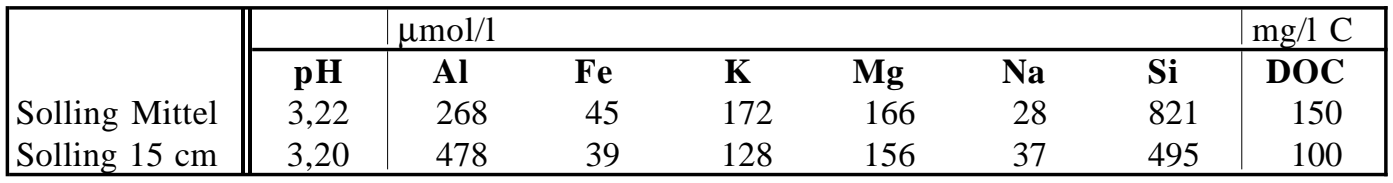

Tab. A-3: Charakterisierung der als Vergleich dienenden abgepreßten Poren-Lösung von einem Quarzit-Ton-Fließerden-Bodenprofil aus dem Harz (Daten aus Schlabach 1996).

\begin{tabular}{|c||cccccc|}
\hline \multicolumn{1}{|c||}{} & & $\mu \mathrm{mol} / \mathbf{l}$ & & & & $\mathrm{mg} / \mathrm{l} \mathrm{C}$ \\
\hline Teufe [cm] & $\mathbf{p H}$ & $\mathbf{A l}$ & $\mathbf{F e}$ & $\mathbf{M g}$ & $\mathbf{S i}$ & $\mathbf{D O C}$ \\
7,5 & 3,33 & 82 & 4,3 & 86 & 196 & n.b. \\
11,5 & 4,21 & 30 & 5,0 & 28 & 55 & 28 \\
15,5 & 4,19 & 12 & 4,3 & 26 & 51 & 37 \\
18,5 & 4,00 & 8,9 & 4,1 & 28 & 54 & 30 \\
21,5 & 4,78 & 7,4 & 4,7 & 23 & 48 & 29 \\
25 & 4,77 & 4,8 & 4,7 & 18 & 57 & 26 \\
29 & 4,48 & 9,6 & 4,7 & 23 & 125 & 30 \\
34 & 4,49 & 7,0 & 3,4 & 15 & 132 & 25 \\
39,5 & 4,72 & 4,1 & 2,7 & 13 & 128 & 16 \\
44,5 & 4,64 & 6,3 & 3,4 & 15 & 142 & 18 \\
52 & 4,96 & $<1,85$ & 0,90 & 2,0 & 167 & 23 \\
\hline
\end{tabular}

Tab. A-4: Chemische Charakterisierung der Mineralproben.

\begin{tabular}{|l||c|c|c|c|c|c|c|c|c|c|}
\hline \multirow{2}{*}{ Probe } & \multicolumn{1}{|c|}{ Gew.- \% } & $\mathbf{N a}_{2}$ & $\mathbf{S i O}_{2} \mathbf{O}_{3}$ & $\mathbf{M n O}$ & $\mathbf{M g O}$ & $\mathbf{C a O}$ & $\mathbf{N a}_{2} \mathbf{O}$ & $\mathbf{K}_{2} \mathbf{O}$ & $\mathbf{P}_{2} \mathbf{O}_{\mathbf{5}}$ \\
\hline \hline Kaolinit & 46,3 & 0,202 & 37,03 & 0,74 & 0,008 & 0,29 & 0,14 & 0,40 & 0,99 & 0,046 \\
Montmorillonit & 60,3 & 0,127 & 21,0 & 3,69 & 0,001 & 2,38 & 0,33 & 2,23 & 0,04 & 0,03 \\
Serizit & 64,7 & 0,649 & 19,8 & 2,75 & 0,01 & 2,34 & 0,21 & 0,27 & 7,76 & 0,13 \\
Illit H-35 & 56,9 & 1,260 & 20,0 & 5,69 & 0,03 & 2,08 & 0,71 & 0,49 & 4,72 & 0,19 \\
Illit OECD & 53,3 & 0,681 & 19,8 & 7,38 & 0,04 & 4,18 & 1,31 & 0,21 & 6,74 & 0,48 \\
Talk & 63,7 & 0,089 & 0,8 & 0,89 & 0,01 & 30,4 & 0,43 & 0,01 & 0,02 & 0,16 \\
\hline
\end{tabular}


In den folgenden Tabellen werden für die Rubrik „Tage” immer zwei Dezimalstellen angegeben.

Das entsricht einer Genauigkeit der Angabe auf 15 Minuten (0,01 Tag $\approx 15$ Minuten).

\section{Kaolinit}

Tab. A-5: Batch-Experimente mit Kaolinit Versuche 1 und 2.

1

Kaolinit

$\emptyset=0,63-2 \mu \mathrm{m}$

Einwaage Mineral [g]: 0,0502

Einwaage Lösung [g]: 100,0333

Lösung: $\mathbf{H}_{2} \mathrm{SO}_{4}, \mathbf{p H}=\mathbf{1}$

\begin{tabular}{|l|r|r|r|r|}
\hline & & \multicolumn{3}{|c|}{$\mu \mathrm{mol} / 1$} \\
\hline \hline $1-1$ & Tage & pH & $\mathbf{A ~ l}$ & $\mathbf{S ~ i}$ \\
\hline $1-2$ & 7,22 & 1,1 & 7,0 & $<0,36$ \\
\hline $1-3$ & 12,99 & 1,2 & 6,7 & 3,1 \\
\hline $1-4$ & 21,17 & 1,1 & 8,2 & 5,1 \\
\hline $1-5$ & 28,16 & 1,2 & 9,9 & 6,8 \\
\hline $1-6$ & 35,31 & 1,4 & 11 & 8,2 \\
\hline $1-7$ & 42,22 & 1,8 & 12 & 9,3 \\
\hline $1-8$ & 49,07 & 1,3 & 13 & 11 \\
\hline $1-9$ & 56,03 & 1,4 & 13 & 12 \\
\hline $1-10$ & 84,02 & 1,2 & 17 & 18 \\
\hline $1-11$ & 91,02 & 1,2 & 17 & 17 \\
\hline $1-12$ & 224,11 & 1,4 & 33 & 38 \\
\hline $1-13$ & 329,20 & 1,5 & 49 & 56 \\
\hline $1-14$ & 349,20 & 1,5 & 45 & 56 \\
\hline $1-15$ & 407,10 & 1,0 & 44 & 49 \\
\hline $1-16$ & 452,16 & 1,2 & 64 & 67 \\
\hline $1-17$ & 494,05 & 1,0 & 65 & 66 \\
\hline & 646,03 & 1,0 & 85 & 83 \\
\hline Rate [mol/g•h)] & & & & \\
\hline
\end{tabular}

2

Kaolinit

$\varnothing=0,63-2 \mu \mathrm{m}$

Einwaage Mineral [g]: 0,0504

Einwaage Lösung [g]: 100,0635

Lösung: $\mathrm{H}_{2} \mathrm{SO}_{4}, \mathbf{p H}=\mathbf{2}$

\begin{tabular}{|c|c|c|c|c|}
\hline \multirow[b]{2}{*}{ Probe } & \multirow[b]{2}{*}{ Tage } & \multirow[b]{2}{*}{ pH } & \multicolumn{2}{|l|}{$\mu \mathrm{mol} / \mathrm{l}$} \\
\hline & & & Al & $\mathbf{S}$ i \\
\hline $2-1$ & $\overline{7,67}$ & $\overline{2,0}$ & $2<0,37$ & $\overline{c<0,36}$ \\
\hline $2-2$ & 13,43 & 2,1 & 0,67 & $<0,36$ \\
\hline $2-3$ & 21,61 & 2,0 & $<0,37$ & 0,67 \\
\hline $2-4$ & 28,61 & 2,0 & $<0,37$ & 1,1 \\
\hline $2-5$ & 35,76 & 2,0 & 0,96 & 1,7 \\
\hline $2-6$ & 42,67 & 2,1 & 0,46 & 1,9 \\
\hline $2-7$ & 49,52 & 2,3 & 1,3 & 2,8 \\
\hline $2-8$ & 56,48 & 2,1 & 0,85 & 3,5 \\
\hline $2-9$ & 84,47 & 2,2 & 1,8 & 7,0 \\
\hline $2-10$ & 91,47 & 1,9 & 2,0 & 7,1 \\
\hline $2-11$ & 224,56 & 2,2 & 4,2 & 15 \\
\hline $2-12$ & 329,65 & 2,2 & 7,2 & 18 \\
\hline $2-13$ & 349,65 & 2,0 & 12 & 20 \\
\hline $2-14$ & 407,55 & 2,0 & 8,5 & 18 \\
\hline $2-15$ & 452,60 & 2,0 & 13 & 24 \\
\hline $2-16$ & 494,50 & 1,9 & 15 & 24 \\
\hline $2-17$ & 646,48 & 2,0 & 28 & 42 \\
\hline \multicolumn{2}{|c|}{ Rate $[\mathrm{mol} /(\mathrm{g} \bullet \mathrm{h})]$} & & $3,3 \cdot 10^{-9}$ & $4,6 \cdot 10^{-9}$ \\
\hline
\end{tabular}


Tab. A-6: Batch-Experimente mit Kaolinit Versuche 3 und 4.

3

\section{Kaolinit}

$\varnothing=0,63-2 \mu \mathrm{m}$

Einwaage Mineral [g]: 0,0504

Einwaage Lösung [g]: 100,0080

Lösung: $\mathbf{H}_{2} \mathrm{SO}_{4}, \mathbf{p H}=\mathbf{3}$

\begin{tabular}{|c|c|c|c|c|}
\hline & & & $\mu \mathrm{mol} / 1$ & \\
\hline Probe & Tage & pH & Al & $\mathbf{S i}$ \\
\hline $3-1$ & 7,22 & $\overline{2,9}$ & \begin{tabular}{c|}
$<0,37$ \\
\end{tabular} & $<0,36$ \\
\hline $3-2$ & 12,99 & 3,0 & $<0,37$ & $<0,36$ \\
\hline $3-3$ & 21,17 & 3,0 & $<0,37$ & $<0,36$ \\
\hline $3-4$ & 28,16 & 3,0 & $<0,37$ & $<0,36$ \\
\hline $3-5$ & 35,31 & 2,9 & $<0,37$ & $<0,36$ \\
\hline $3-6$ & 42,22 & 3,0 & $<0,37$ & $<0,36$ \\
\hline $3-7$ & 49,07 & 3,1 & $<0,37$ & $<0,36$ \\
\hline $3-8$ & 56,03 & 3,0 & $<0,37$ & 0,62 \\
\hline $3-9$ & 84,02 & 2,9 & $<0,37$ & 2,1 \\
\hline $3-10$ & 91,02 & 2,9 & $<0,37$ & 1,3 \\
\hline $3-11$ & 224,11 & 3,1 & $<0,37$ & 7,2 \\
\hline $3-12$ & 329,20 & 2,9 & $<0,37$ & 7,9 \\
\hline $3-13$ & 349,20 & 2,7 & $<0,37$ & 8,4 \\
\hline $3-14$ & 407,10 & 2,8 & $<0,37$ & 7,3 \\
\hline $3-15$ & 452,16 & 2,7 & 0,39 & 9,5 \\
\hline $3-16$ & 494,05 & 2,7 & 0,48 & 9,2 \\
\hline $3-17$ & 646,03 & 2,8 & 0,90 & 17 \\
\hline $3-18$ & 726,09 & 2,8 & 1,7 & 19 \\
\hline 3-19 & 849,00 & 2,8 & 1,4 & 20 \\
\hline \multicolumn{2}{|c|}{ Rate $[\mathrm{mol} /(\mathrm{g} \bullet \mathrm{h})]$} & & $2,6 \cdot 10^{-10}$ & $2,0 \cdot 10^{-9}$ \\
\hline
\end{tabular}

4

\section{Kaolinit}

$\varnothing=0,63-2 \mu \mathrm{m}$

Einwaage Mineral [g]: 0,0501

Einwaage Lösung [g]: 100,0209

Lösung: $\mathrm{H}_{2} \mathrm{SO}_{4}, \mathbf{p H}=\mathbf{4}$

\begin{tabular}{|c|c|c|c|c|}
\hline & & & $\mu \mathrm{mol} / 1$ & \\
\hline Probe & Tage & $\mathbf{p H}$ & Al & $\mathbf{S i}$ \\
\hline $4-1$ & 7,22 & $\overline{3,9}$ & $<0,37$ & 1,9 \\
\hline $4-2$ & 12,99 & 4,0 & $<0,37$ & 3,4 \\
\hline $4-3$ & 21,17 & 4,0 & $<0,37$ & 5,1 \\
\hline $4-4$ & 28,16 & 3,9 & $<0,37$ & 3,3 \\
\hline $4-5$ & 35,31 & 3,8 & $<0,37$ & 3,2 \\
\hline $4-6$ & 42,22 & 4,0 & $<0,37$ & 2,6 \\
\hline 4-7 & 49,07 & 4,0 & $<0,37$ & 3,3 \\
\hline $4-8$ & 56,03 & 3,9 & $<0,37$ & 3,3 \\
\hline $4-9$ & 224,11 & 3,9 & $<0,37$ & 3,8 \\
\hline $4-10$ & 329,20 & 3,6 & $<0,37$ & 3,7 \\
\hline $4-11$ & 349,20 & 3,4 & $<0,37$ & 3,9 \\
\hline $4-12$ & 407,10 & 3,4 & $<0,37$ & 3,3 \\
\hline 4-13 & 452,16 & 3,3 & $<0,37$ & 4,5 \\
\hline 4-14 & 494,05 & 3,3 & $<0,37$ & 4,5 \\
\hline 4-15 & 646,04 & 3,7 & $<0,37$ & 10 \\
\hline 4-16 & 726,09 & 3,4 & 1,0 & 11 \\
\hline 4-17 & 849,00 & 3,6 & $<0,37$ & 10 \\
\hline \multicolumn{2}{|c|}{ Rate $[\mathrm{mol} /(\mathrm{g} \bullet \mathrm{h})]$} & & & $1,3 \cdot 10^{-9}$ \\
\hline
\end{tabular}


Tab. A-7: Batch-Experimente mit Kaolinit Versuche 5 und 7.

5

\section{Kaolinit}

$\varnothing=0,63-2 \mu \mathrm{m}$

Einwaage Mineral [g]: 0,0503

Einwaage Lösung [g]: 100,0251

Lösung: $\mathbf{H}_{2} \mathrm{O}^{* * * * *}, \mathbf{p H}>\mathbf{4}$

\begin{tabular}{|c|c|c|c|c|}
\hline & & & $\mu \mathrm{mol} / \mathrm{l}$ & \\
\hline Probe & Tage & pH & Al & Si \\
\hline $5-1$ & $\overline{7,22}$ & $\overline{4,4}$ & $<0,37$ & $\overline{1,0}$ \\
\hline $5-2$ & 12,99 & 4,9 & $<0,37$ & 1,5 \\
\hline $5-3$ & 21,17 & 4,9 & $<0,37$ & 4,2 \\
\hline $5-4$ & 28,16 & 4,6 & $<0,37$ & 2,5 \\
\hline $5-5$ & 35,31 & 4,6 & $<0,37$ & 2,0 \\
\hline $5-6$ & 42,22 & 4,3 & $<0,37$ & 1,8 \\
\hline $5-7$ & 49,07 & 4,5 & 0,71 & 2,5 \\
\hline $5-8$ & 56,03 & 4,5 & $<0,37$ & 2,4 \\
\hline $5-9$ & 224,11 & 4,4 & $<0,37$ & 2,7 \\
\hline $5-10$ & 349,20 & 3,7 & $<0,37$ & 3,2 \\
\hline $5-11$ & 407,10 & 3,9 & $<0,37$ & 2,4 \\
\hline $5-12$ & 452,16 & 3,9 & 1,1 & 4,2 \\
\hline $5-13$ & 494,05 & 4,0 & 1,5 & 4,5 \\
\hline $5-14$ & 646,04 & 4,0 & 0,78 & 8,9 \\
\hline $5-15$ & 726,09 & 4,3 & 1,0 & 9,1 \\
\hline \multicolumn{2}{|c|}{ Rate $[\mathrm{mol} /(\mathrm{g} \bullet \mathrm{h})]$} & & $2,3 \cdot 10^{-10}$ & $1,7 \cdot 10^{-9}$ \\
\hline
\end{tabular}

7

\section{Kaolinit}

$\varnothing=0,63-2 \mu \mathrm{m}$

Einwaage Mineral [g]: 0,1507

Einwaage Lösung [g]: 100,4055

Lösung: $\mathbf{H}_{2} \mathbf{S O}_{4}, \mathbf{p H}=\mathbf{1}$

\begin{tabular}{|c|c|c|c|c|}
\hline & & & $\mu \mathrm{mol} / 1$ & \\
\hline Probe & Tage & $\mathbf{p H}$ & Al & $\mathbf{S i}$ \\
\hline $7-1$ & $\overline{19,19}$ & $\overline{1,3}$ & 26 & 29 \\
\hline $7-2$ & 40,14 & 1,3 & 39 & 47 \\
\hline $7-3$ & 100,09 & 1,0 & 63 & 72 \\
\hline $7-4$ & 142,18 & 1,3 & 103 & 111 \\
\hline $7-5$ & 184,05 & 1,0 & 115 & 123 \\
\hline $7-6$ & 336,07 & 1,1 & 181 & 185 \\
\hline $7-7$ & 416,06 & 1,0 & 236 & 312 \\
\hline $7-8$ & 538,98 & 1,0 & 295 & 385 \\
\hline \multicolumn{2}{|c|}{ Rate $[\mathrm{mol} /(\mathrm{g} \cdot \mathrm{h})$} & & $1,4 \cdot 10^{-8}$ & $1,9 \cdot 10^{-8}$ \\
\hline
\end{tabular}


Tab. A-8: Batch-Experimente mit Kaolinit Versuche 8 und 18.

8

\section{Kaolinit}

$\varnothing=0,63-2 \mu \mathrm{m}$

Einwaage Mineral [g]: 0,1508

Einwaage Lösung [g]: 100,1168

Lösung: $\mathrm{H}_{2} \mathrm{SO}_{4}, \mathbf{p H}=\mathbf{2}$

\begin{tabular}{|c|c|c|c|c|}
\hline \multirow[b]{2}{*}{ Probe } & \multirow[b]{2}{*}{ Tage } & \multirow[b]{2}{*}{ pH } & \multicolumn{2}{|l|}{$\mu \mathrm{mol} / 1$} \\
\hline & & & Al & $\mathbf{S}$ i \\
\hline $8-1$ & 19,09 & 2,0 & 1,4 & 15 \\
\hline $8-2$ & 40,05 & 1,9 & 2,4 & 21 \\
\hline $8-3$ & 99,99 & 1,9 & 5,8 & 28 \\
\hline $8-4$ & 142,08 & 1,9 & 11 & 39 \\
\hline $8-5$ & 183,95 & 1,9 & 14 & 40 \\
\hline $8-6$ & 335,97 & 1,9 & 30 & 64 \\
\hline $8-7$ & 415,96 & 1,9 & 36 & 88 \\
\hline $8-8$ & 538,88 & 1,9 & 46 & 105 \\
\hline \multicolumn{2}{|c|}{ Rate $[\mathrm{mol} /(\mathrm{g} \bullet \mathrm{h})]$} & & $2,5 \cdot 10^{-9}$ & $4,7 \cdot 10^{-9}$ \\
\hline
\end{tabular}

18

Kaolinit

$\varnothing=0,63-2 \mu \mathrm{m}$

Einwaage Mineral [g]: 0,1504

Einwaage Lösung [g]: 100,0049

Lösung: $\mathrm{C}_{6} \mathrm{H}_{8} \mathrm{O}_{7} \bullet \mathrm{H}_{2} \mathrm{O}, \mathbf{M}=\mathbf{0 , 0 1}$

\begin{tabular}{|c|c|c|c|c|}
\hline \multirow[b]{2}{*}{ Probe } & \multirow[b]{2}{*}{ Tage } & \multirow[b]{2}{*}{ pH } & \multicolumn{2}{|l|}{$\mu \mathrm{mol} / 1$} \\
\hline & & & Al & $\mathbf{S} \mathbf{i}$ \\
\hline $18-1$ & 8,08 & 2,4 & 1,2 & 11 \\
\hline $18-2$ & 29,01 & 2,3 & 2,3 & 18 \\
\hline $18-3$ & 86,01 & 2,7 & 5,9 & 24 \\
\hline $18-4$ & 131,01 & 2,6 & 11 & 35 \\
\hline $18-5$ & 172,90 & 2,6 & 13 & 37 \\
\hline $18-6$ & 187,83 & 2,5 & 15 & 45 \\
\hline $18-7$ & 329,98 & 2,9 & 25 & 51 \\
\hline \multicolumn{2}{|c|}{ Rate $[\mathrm{mol} /(\mathrm{g} \bullet \mathrm{h})]$} & & $2,1 \cdot 10^{-9}$ & $3,5 \cdot 10^{-9}$ \\
\hline
\end{tabular}

Tab. A-9: Batch-Experimente mit Kaolinit Versuch 47. IN kennzeichnet die Input-Lösung.

\section{7}

\section{Kaolinit}

$\varnothing=0,63-2 \mu \mathrm{m}$

Einwaage Mineral [g]: 0,0380

Einwaage Lösung [g]: 30,6407

Lösung: abgepreßte Poren-Lösung Solling

$$
\begin{aligned}
& \text { 0-15 cm Tiefe } \\
& \text { pH }=3,5 \\
& \text { DOC }=39,15 \mathrm{mg} / \mathrm{l}
\end{aligned}
$$

\begin{tabular}{|c|r|r|r|r|r|}
\hline & & \multicolumn{4}{l|}{$\mu \mathrm{mol} / 1$} \\
Probe & Tage & \multicolumn{1}{c|}{$\mathbf{p H}$} & \multicolumn{1}{c|}{ A l } & \multicolumn{1}{c|}{ F e } & \multicolumn{1}{c|}{ S i } \\
\hline \hline IN & 0 & 3,5 & 20 & 6,3 & 362 \\
\hline $47-1$ & 24,99 & 4,9 & 12 & 2,6 & 392 \\
$47-2$ & 62,01 & 5,3 & 8,2 & 2,0 & 429 \\
\hline $47-3$ & 184,03 & 5,7 & 2,0 & 1,5 & 429 \\
\hline
\end{tabular}


Tab. A-10: Durchfluß-Experimente mit Kaolinit Versuch II.

II

\section{Kaolinit}

$\emptyset=0,63-2 \mu \mathrm{m}$

Einwaage Mineral [g]: 0,1806

Einwaage Lösung [g]: 89,0

Lösung: $\mathbf{H}_{2} \mathrm{SO}_{4}, \mathbf{p H}=\mathbf{2}$

\begin{tabular}{|c|c|c|c|c|c|}
\hline & & & & $\mu \mathrm{mol} / \mathrm{l}$ & \\
\hline Probe & Tage & $\mathrm{g} / \mathrm{h}$ & pH & Al & $\mathbf{S i}$ \\
\hline II-1 & 0,13 & 1,12 & 2,1 & 0,55 & 8,4 \\
\hline II-2 & 0,25 & 1,10 & 2,1 & 0,42 & 13 \\
\hline III-3 & 0,90 & 0,61 & 2,1 & $<0,37$ & 18 \\
\hline III-4 & 0,99 & 0,86 & 2,1 & $<0,37$ & 19 \\
\hline II-5 & 1,07 & 1,20 & 2,1 & $<0,37$ & 19 \\
\hline II-6 & 1,15 & 1,09 & 2,1 & $<0,37$ & 19 \\
\hline III-7 & 1,24 & 1,09 & 2,1 & 0,39 & 19 \\
\hline II-8 & 1,90 & 1,08 & 2,1 & $<0,37$ & 19 \\
\hline II-9 & 1,99 & 1,10 & 2,1 & 0,38 & 19 \\
\hline II-10 & 2,07 & 1,09 & 2,1 & $<0,37$ & 18 \\
\hline II-11 & 2,15 & 1,09 & 2,1 & $<0,37$ & 17 \\
\hline II-12 & 2,97 & 1,08 & 2,1 & 0,38 & 19 \\
\hline II-13 & 3,10 & 1,09 & 2,1 & 0,49 & 23 \\
\hline II-14 & 3,97 & 1,08 & 2,1 & 0,44 & 22 \\
\hline II-15 & 4,09 & 1,10 & 2,1 & 0,54 & 21 \\
\hline II-16 & 4,93 & 1,08 & 2,1 & 0,51 & 21 \\
\hline II-17 & 5,07 & 1,09 & 2,1 & 0,52 & 19 \\
\hline II-18 & 5,20 & 1,08 & 2,1 & 0,52 & 19 \\
\hline II-19 & 5,92 & 1,07 & 2,1 & 0,51 & 18 \\
\hline III-20 & 6,11 & 1,09 & 2,1 & 0,56 & 18 \\
\hline II-21 & 6,34 & 1,08 & 2,1 & 0,53 & 15 \\
\hline III-22 & 6,97 & 1,08 & 2,1 & 0,56 & 14 \\
\hline III-23 & 7,13 & 1,08 & 2,1 & 0,59 & 14 \\
\hline III-24 & 7,31 & 1,09 & 2,1 & 0,57 & 14 \\
\hline III-25 & 7,99 & 1,07 & 2,1 & 0,59 & 13 \\
\hline II-26 & 8,28 & 1,08 & 2,1 & 0,60 & 12 \\
\hline II-27 & 8,93 & 1,08 & 2,1 & 0,67 & 11 \\
\hline II-28 & 9,22 & 1,08 & 2,1 & 0,65 & 11 \\
\hline II-29 & 10,31 & 1,08 & 2,1 & 0,76 & 12 \\
\hline III-30 & 11,21 & 1,07 & 2,1 & 0,83 & 11 \\
\hline II-31 & 11,96 & 1,07 & 2,1 & 0,91 & 9,8 \\
\hline III-32 & 12,29 & 1,07 & 2,1 & 0,97 & 9,4 \\
\hline II-33 & 13,01 & 1,07 & 2,1 & 1,0 & 10 \\
\hline III-34 & 13,20 & 1,07 & 2,1 & 1,1 & 9,5 \\
\hline II-35 & 13,97 & 1,07 & 2,1 & 1,2 & 9,3 \\
\hline II-36 & 14,17 & 1,09 & 2,1 & 1,2 & 8,2 \\
\hline II-37 & 14,94 & 1,07 & 2,1 & 1,3 & 7,9 \\
\hline II-38 & 15,19 & 1,07 & 2,1 & 1,4 & 8,7 \\
\hline II-39 & 16,07 & 1,07 & 2,0 & 1,5 & 8,0 \\
\hline II-40 & 16,19 & 1,07 & 2,0 & 1,6 & 6,5 \\
\hline
\end{tabular}

\begin{tabular}{|c|c|c|c|c|c|}
\hline & & & & $\mu \mathrm{mol} / \mathrm{l}$ & \\
\hline Probe & Tage & $\mathrm{g} / \mathrm{h}$ & pH & $\overline{A l}$ & $\mathbf{S i}$ \\
\hline II-41 & 17,15 & 1,07 & 2,0 & 1,7 & 6,3 \\
\hline II-42 & 18,92 & 1,07 & 2,0 & 1,9 & 5,8 \\
\hline II-43 & 19,28 & 1,07 & 2,0 & 2,0 & 5,5 \\
\hline II-44 & 20,21 & 1,07 & 2,0 & 2,2 & 5,2 \\
\hline II-45 & 21,95 & 1,07 & 2,0 & 2,2 & 5,6 \\
\hline II-46 & 23,30 & 1,06 & 2,0 & 2,3 & 5,3 \\
\hline II-47 & 24,11 & 1,05 & 2,0 & 2,3 & 5,5 \\
\hline II-48 & 25,93 & 1,05 & 2,0 & 2,3 & 5,1 \\
\hline II-49 & 27,21 & 1,05 & 2,0 & 2,3 & 4,5 \\
\hline II-50 & 28,19 & 1,05 & 2,0 & 2,3 & 4,6 \\
\hline II-51 & 30,25 & 1,04 & 2,0 & 2,4 & 4,2 \\
\hline II-52 & 32,91 & 0,64 & 2,0 & 2,3 & 4,5 \\
\hline II-53 & 33,96 & 1,04 & 2,0 & 2,4 & 5,1 \\
\hline II-54 & 42,08 & 1,04 & 2,0 & 2,1 & 3,8 \\
\hline II-55 & 44,06 & 0,20 & 2,0 & 2,1 & 3,1 \\
\hline II-56 & 46,93 & 0,59 & 2,0 & 3,1 & 3,3 \\
\hline II-57 & 48,96 & 0,94 & 2,0 & 2,3 & 3,3 \\
\hline II-58 & 51,06 & 0,98 & 2,1 & 2,4 & 4,0 \\
\hline II-59 & 53,06 & 0,98 & 2,1 & 2,5 & 4,2 \\
\hline II-60 & 55,20 & 0,97 & 2,0 & 2,8 & 3,9 \\
\hline II-61 & 57,21 & 0,98 & 2,1 & 3,3 & 3,7 \\
\hline II-62 & 59,22 & 0,99 & 2,0 & 3,8 & 5,5 \\
\hline II-63 & 61,23 & 1,04 & 1,9 & 4,0 & 5,2 \\
\hline II-64 & 63,39 & 1,02 & 1,9 & 4,3 & 3,7 \\
\hline II-65 & 65,15 & 1,03 & 2,1 & 4,1 & 5,4 \\
\hline II-66 & 67,21 & 1,02 & 2,0 & 4,2 & 5,6 \\
\hline II-67 & 69,14 & 1,05 & 2,1 & 3,9 & 5,1 \\
\hline II-68 & 71,28 & 1,17 & 2,1 & 3,6 & 4,8 \\
\hline II-69 & 73,02 & 1,05 & 2,1 & 3,5 & 4,1 \\
\hline II-70 & 75,12 & 1,06 & 2,1 & 3,5 & 4,6 \\
\hline II-71 & 77,20 & 1,05 & 2,1 & 3,1 & 3,8 \\
\hline \multicolumn{2}{|c|}{ Rate $[\mathrm{mol} /(\mathrm{g} \bullet \mathrm{h})]$} & & & $2,2 \cdot 10^{-8}$ & $2,8 \cdot 10^{-8}$ \\
\hline
\end{tabular}


Tab. A-11: Durchfluß-Experimente mit Kaolinit Versuch IV90(1).

\section{IV90(1)}

\section{Kaolinit}

$\varnothing=0,63-2 \mu \mathrm{m}$

Einwaage Mineral [g]: 0,2704

Einwaage Lösung [g]: 90,7

Lösung: $\mathrm{H}_{2} \mathrm{SO}_{4}, \mathbf{p H}=\mathbf{1}$

\begin{tabular}{|l|r|r|r|r|r|}
\hline & & & \multicolumn{1}{|c|}{$\boldsymbol{\mu m o l} / \mathbf{l}$} \\
\hline Probe & Tage & g/h & pH & Al & Si \\
\hline \hline IV90(1)-1 & 1,08 & 0,92 & 1,1 & 14 & 13 \\
\hline IV90(1)-2 & 3,12 & 0,91 & 1,1 & 19 & 20 \\
\hline IV90(1)-3 & 4,08 & 0,90 & 0,8 & 20 & 23 \\
\hline IV90(1)-4 & 4,99 & 0,91 & 0,8 & 21 & 23 \\
\hline IV90(1)-5 & 6,14 & 0,90 & 0,8 & 21 & 23 \\
\hline IV90(1)-6 & 7,18 & 0,90 & 0,8 & 20 & 23 \\
\hline IV90(1)-7 & 8,16 & 0,90 & 0,8 & 21 & 23 \\
\hline IV90(1)-8 & 9,06 & 0,91 & 0,9 & 21 & 23 \\
\hline IV90(1)-9 & 10,16 & 0,91 & 0,9 & 21 & 23 \\
\hline IV90(1)-10 & 11,07 & 0,91 & 0,8 & 20 & 23 \\
\hline IV90(1)-11 & 11,97 & 0,91 & 0,9 & 21 & 23 \\
\hline IV90(1)-12 & 13,11 & 0,91 & 0,9 & 20 & 22 \\
\hline IV90(1)-13 & 14,16 & 0,91 & 0,9 & 21 & 23 \\
\hline IV90(1)-14 & 15,00 & 0,92 & 0,9 & 21 & 23 \\
\hline IV90(1)-15 & 16,05 & 0,92 & 0,8 & 21 & 22 \\
\hline IV90(1)-16 & 17,00 & 0,91 & 0,8 & 21 & 22 \\
\hline IV90(1)-17 & 17,86 & 0,91 & 0,8 & 21 & 22 \\
\hline IV90(1)-18 & 20,14 & 0,91 & 0,8 & 21 & 22 \\
\hline IV90(1)-19 & 21,13 & 0,90 & 0,8 & 21 & 22 \\
\hline IV90(1)-20 & 22,04 & 0,91 & 0,8 & 21 & 22 \\
\hline IV90(1)-21 & 23,03 & 0,91 & 0,8 & 22 & 23 \\
\hline IV90(1)-22 & 23,98 & 0,91 & 1,1 & 22 & 23 \\
\hline IV90(1)-23 & 24,85 & 0,91 & 1,1 & 22 & 24 \\
\hline IV90(1)-24 & 25,88 & 0,91 & 1,1 & 23 & 24 \\
\hline IV90(1)-25 & 26,88 & 0,91 & 1,1 & 24 & 26 \\
\hline IV90(1)-26 & 27,88 & 0,91 & 1,1 & 26 & 26 \\
\hline IV90(1)-27 & 28,98 & 0,88 & 1,0 & 25 & 25 \\
\hline IV90(1)-28 & 29,89 & 0,94 & 1,0 & 26 & 27 \\
\hline IV90(1)-29 & 30,88 & 0,94 & 1,0 & 26 & 27 \\
\hline IV90(1)-30 & 31,84 & 0,94 & 1,0 & 26 & 27 \\
\hline IV90(1)-31 & 32,87 & 0,94 & 1,0 & 25 & 26 \\
\hline IV90(1)-32 & 33,91 & 0,92 & 1,0 & 25 & 26 \\
\hline IV90(1)-33 & 34,90 & 0,93 & 1,0 & 26 & 26 \\
\hline IV90(1)-34 & 35,88 & 0,94 & 0,9 & 26 & 27 \\
\hline IV90(1)-35 & 36,88 & 0,95 & 0,9 & 27 & 28 \\
\hline IV90(1)-36 & 37,83 & 0,93 & 1,0 & 28 & 29 \\
\hline IV90(1)-37 & 42,94 & 0,93 & 1,0 & 29 & 30 \\
\hline IV90(1)-38 & 43,89 & 0,93 & 1,0 & 30 & 31 \\
\hline IV90(1)-39 & 44,90 & 0,93 & 1,0 & 30 & 31 \\
\hline IV90(1)-40 & 45,89 & 0,93 & 1,0 & 29 & 31 \\
\hline
\end{tabular}

\begin{tabular}{|c|r|r|r|r|r|}
\hline & & & \multicolumn{1}{|c|}{$\mu \mathrm{mol} / 1$} \\
\hline Probe & Tage & g/h & \multicolumn{1}{|c|}{$\mathbf{p H}$} & \multicolumn{1}{c|}{ Al } & \multicolumn{1}{c|}{$\mathbf{S i}$} \\
\hline \hline IV90(1)-41 & 46,87 & 0,93 & 1,0 & 28 & 31 \\
\hline IV90(1)-42 & 47,88 & 0,93 & 1,0 & 29 & 31 \\
\hline IV90(1)-43 & 48,84 & 0,96 & 1,0 & 29 & 31 \\
\hline IV90(1)-44 & 49,87 & 0,94 & 1,0 & 29 & 32 \\
\hline IV90(1)-45 & 50,87 & 0,91 & 1,0 & 30 & 32 \\
\hline IV90(1)-46 & 51,88 & 0,92 & 1,0 & 30 & 32 \\
\hline IV90(1)-47 & 52,88 & 0,91 & 1,0 & 28 & 31 \\
\hline IV90(1)-48 & 55,01 & 0,77 & 1,0 & 29 & 32 \\
\hline IV90(1)-49 & 69,92 & 0,74 & 1,1 & 27 & 29 \\
\hline & & & & & \\
\hline \multicolumn{2}{|l|}{ Rate [mol/(g•h)] } & & & $9,5 \bullet 10^{-8}$ & $1,0 \bullet 10^{-7}$ \\
\hline
\end{tabular}


Tab. A-12: Durchfluß-Experimente mit Kaolinit Versuch IV90(2).

\section{IV90(2)}

\section{Kaolinit}

$\varnothing=0,63-2 \mu \mathrm{m}$

Einwaage Mineral [g]: 0,2704

Einwaage Lösung [g]: 90,7

Lösung: $\mathrm{H}_{2} \mathrm{SO}_{4}, \mathbf{p H}=\mathbf{2}$

\begin{tabular}{|l|r|r|r|r|r|}
\hline & & & \multicolumn{1}{|c|}{$\boldsymbol{\mu m o l} / 1$} \\
\hline Probe & Tage & g/h & pH & Al & Si \\
\hline \hline IV90(2)-1 & 25,02 & 0,52 & 2,0 & 9,8 & 27 \\
\hline IV90(2)-2 & 26,14 & 0,52 & 2,0 & 9,3 & 27 \\
\hline IV90(2)-3 & 26,98 & 0,51 & 2,0 & 9,3 & 26 \\
\hline IV90(2)-4 & 28,07 & 0,51 & 2,1 & 9,2 & 26 \\
\hline IV90(2)-5 & 29,06 & 0,51 & 2,1 & 9,1 & 25 \\
\hline IV90(2)-6 & 30,17 & 0,51 & 2,1 & 9,1 & 25 \\
\hline IV90(2)-7 & 47,09 & 0,22 & 2,1 & 19 & 30 \\
\hline IV90(2)-8 & 49,07 & 0,09 & 2,0 & 19 & 30 \\
\hline IV90(2)-9 & 50,06 & 0,21 & 2,1 & 20 & 30 \\
\hline IV90(2)-10 & 51,78 & 0,29 & 2,1 & 20 & 28 \\
\hline IV90(2)-11 & 52,97 & 0,15 & 2,1 & 20 & 28 \\
\hline IV90(2)-12 & 54,19 & 0,16 & 2,1 & 20 & 27 \\
\hline IV90(2)-13 & 56,09 & 0,23 & 2,1 & 20 & 27 \\
\hline IV90(2)-14 & 57,93 & 0,30 & 2,1 & 19 & 26 \\
\hline IV90(2)-15 & 59,98 & 0,30 & 2,1 & 19 & 24 \\
\hline IV90(2)-16 & 61,25 & 0,24 & 2,1 & 19 & 24 \\
\hline IV90(2)-17 & 61,96 & 0,11 & 2,1 & 18 & 25 \\
\hline IV90(2)-18 & 63,19 & 0,22 & 2,1 & 19 & 24 \\
\hline IV90(2)-19 & 64,10 & 0,16 & 2,0 & 19 & 24 \\
\hline IV90(2)-20 & 65,15 & 0,22 & 2,1 & 18 & 23 \\
\hline IV90(2)-21 & 66,77 & 0,26 & 2,1 & 18 & 23 \\
\hline IV90(2)-22 & 68,91 & 0,29 & 2,1 & 17 & 23 \\
\hline IV90(2)-23 & 70,77 & 0,31 & 2,1 & 17 & 22 \\
\hline IV90(2)-24 & 72,80 & 0,35 & 2,1 & 17 & 23 \\
\hline IV90(2)-25 & 74,12 & 0,49 & 2,1 & 17 & 23 \\
\hline IV90(2)-26 & 75,88 & 0,48 & 2,1 & 18 & 23 \\
\hline IV90(2)-27 & 77,15 & 0,48 & 2,1 & 19 & 23 \\
\hline IV90(2)-28 & 78,08 & 0,48 & 2,1 & 19 & 23 \\
\hline IV90(2)-29 & 81,93 & 0,19 & 2,1 & 22 & 27 \\
\hline IV90(2)-30 & 84,03 & 0,37 & 2,1 & 25 & 30 \\
\hline IV90(2)-31 & 85,94 & 0,39 & 2,1 & 25 & 30 \\
\hline IV90(2)-32 & 87,98 & 0,40 & 2,1 & 25 & 29 \\
\hline IV90(2)-33 & 89,90 & 0,39 & 2,1 & 25 & 29 \\
\hline IV90(2)-34 & 91,81 & 0,41 & 2,0 & 25 & 29 \\
\hline IV90(2)-35 & 93,89 & 0,45 & 2,0 & 26 & 29 \\
\hline IV90(2)-36 & 95,93 & 0,46 & 2,1 & 23 & 29 \\
\hline IV90(2)-37 & 97,84 & 0,47 & 2,0 & 24 & 29 \\
\hline IV90(2)-38 & 99,90 & 0,48 & 2,0 & 21 & 26 \\
\hline IV90(2)-39 & 101,81 & 0,43 & 2,0 & 23 & 28 \\
\hline IV90(2)-40 & 103,92 & 0,39 & 2,0 & 23 & 28 \\
\hline
\end{tabular}

\begin{tabular}{|c|c|c|c|c|c|}
\hline & & & & $\mu \mathrm{mol} / 1$ & \\
\hline Probe & Tage & $\mathrm{g} / \mathrm{h}$ & $\mathbf{p H}$ & $\mathbf{A l}$ & $\mathbf{S i}$ \\
\hline IV90(2)-41 & 105,82 & 0,33 & 2,0 & 24 & 29 \\
\hline IV90(2)-42 & 107,83 & 0,35 & 2,0 & 24 & 30 \\
\hline IV90(2)-43 & 109,03 & 0,31 & 2,0 & 25 & 30 \\
\hline IV90(2)-44 & 119,89 & 0,34 & 2,0 & 24 & 31 \\
\hline IV90(2)-45 & 121,16 & 0,38 & 2,1 & 23 & 30 \\
\hline IV90(2)-46 & 141,07 & 0,39 & 2,1 & 21 & 29 \\
\hline IV90(2)-47 & 142,84 & 0,41 & 2,1 & 21 & 26 \\
\hline IV90(2)-48 & 144,99 & 0,38 & 2,1 & 21 & 27 \\
\hline IV90(2)-49 & 146,92 & 0,38 & 2,1 & 22 & 27 \\
\hline IV90(2)-50 & 148,86 & 0,34 & 2,1 & 22 & 27 \\
\hline IV90(2)-51 & 151,00 & 0,33 & 2,0 & 22 & 27 \\
\hline IV90(2)-52 & 152,83 & 0,37 & 2,0 & 23 & 28 \\
\hline IV90(2)-53 & 154,90 & 0,39 & 2,0 & 23 & 28 \\
\hline IV90(2)-54 & 156,98 & 0,39 & 2,0 & 22 & 28 \\
\hline IV90(2)-55 & 159,01 & 0,34 & 2,0 & 23 & 28 \\
\hline IV90(2)-56 & 161,02 & 0,31 & 2,0 & 23 & 28 \\
\hline IV90(2)-57 & 163,11 & 0,32 & 2,1 & 23 & 28 \\
\hline IV90(2)-58 & 165,00 & 0,33 & 2,1 & 24 & 28 \\
\hline IV90(2)-59 & 167,21 & 0,38 & 2,1 & 24 & 28 \\
\hline IV90(2)-60 & 171,19 & 0,29 & 2,1 & 24 & 28 \\
\hline IV90(2)-61 & 173,19 & 0,32 & 2,1 & 24 & 28 \\
\hline IV90(2)-62 & 175,14 & 0,33 & 2,1 & 24 & 28 \\
\hline IV90(2)-63 & 177,23 & 0,34 & 2,1 & 24 & 28 \\
\hline \multicolumn{2}{|c|}{ Rate $[\mathrm{mol} /(\mathrm{g} \bullet \mathrm{h})]$} & & & $2,9 \cdot 10^{-8}$ & $3,5 \cdot 10^{-8}$ \\
\hline
\end{tabular}


Tab. A-13: Durchfluß-Experimente mit Kaolinit Versuch IV250(1).

\section{IV250(1)}

\section{Kaolinit}

$\varnothing=0,63-2 \mu \mathrm{m}$

Einwaage Mineral [g]: 0,7506

Einwaage Lösung [g]: 249,3

Lösung: $\mathrm{H}_{2} \mathrm{SO}_{4}, \mathbf{p H}=\mathbf{1}$

\begin{tabular}{|c|r|r|r|r|r|}
\hline & & & \multicolumn{2}{|c|}{$\boldsymbol{\mu m o l} / \mathbf{l}$} \\
\hline Probe & Tage & g/h & pH & Al & Si \\
\hline \hline IV250(1)-1 & 1,08 & 0,96 & 1,1 & 16 & 15 \\
\hline IV250(1)-2 & 3,12 & 0,93 & 1,1 & 23 & 25 \\
\hline IV250(1)-3 & 4,08 & 0,93 & 0,8 & 27 & 30 \\
\hline IV250(1)-4 & 4,99 & 0,94 & 0,8 & 28 & 32 \\
\hline IV250(1)-5 & 6,14 & 0,93 & 0,8 & 30 & 34 \\
\hline IV250(1)-6 & 7,18 & 0,93 & 0,8 & 31 & 36 \\
\hline IV250(1)-7 & 8,16 & 0,94 & 0,8 & 32 & 37 \\
\hline IV250(1)-8 & 9,06 & 0,96 & 0,8 & 33 & 38 \\
\hline IV250(1)-9 & 10,16 & 0,95 & 0,8 & 34 & 39 \\
\hline IV250(1)-10 & 11,07 & 0,95 & 0,9 & 35 & 40 \\
\hline IV250(1)-11 & 11,97 & 0,96 & 0,9 & 35 & 40 \\
\hline IV250(1)-12 & 13,11 & 0,95 & 0,9 & 36 & 41 \\
\hline IV250(1)-13 & 14,16 & 0,96 & 0,9 & 36 & 41 \\
\hline IV250(1)-14 & 15,00 & 0,98 & 0,9 & 38 & 43 \\
\hline IV250(1)-15 & 16,05 & 0,97 & 0,9 & 40 & 45 \\
\hline IV250(1)-16 & 17,00 & 0,94 & 0,9 & 40 & 45 \\
\hline IV250(1)-17 & 17,86 & 0,96 & 0,9 & 41 & 46 \\
\hline IV250(1)-18 & 20,14 & 0,95 & 0,9 & 42 & 47 \\
\hline IV250(1)-19 & 21,13 & 0,94 & 0,9 & 43 & 48 \\
\hline IV250(1)-20 & 22,04 & 0,95 & 0,9 & 43 & 48 \\
\hline IV250(1)-21 & 23,03 & 0,96 & 0,9 & 44 & 48 \\
\hline IV250(1)-22 & 23,98 & 0,96 & 1,1 & 45 & 49 \\
\hline IV250(1)-23 & 24,85 & 0,96 & 1,1 & 45 & 50 \\
\hline IV250(1)-24 & 25,88 & 0,95 & 1,1 & 45 & 49 \\
\hline IV250(1)-25 & 26,88 & 0,96 & 1,1 & 46 & 50 \\
\hline IV250(1)-26 & 27,88 & 0,97 & 1,1 & 49 & 52 \\
\hline IV250(1)-27 & 28,98 & 0,88 & 1,0 & 44 & 46 \\
\hline IV250(1)-28 & 29,89 & 0,99 & 1,0 & 45 & 47 \\
\hline IV250(1)-29 & 30,88 & 0,98 & 1,0 & 45 & 48 \\
\hline IV250(1)-30 & 31,84 & 0,99 & 1,0 & 45 & 48 \\
\hline IV250(1)-31 & 32,87 & 0,94 & 1,0 & 46 & 48 \\
\hline IV250(1)-32 & 33,91 & 0,92 & 1,0 & 45 & 48 \\
\hline IV250(1)-33 & 34,90 & 0,98 & 1,0 & 46 & 48 \\
\hline IV250(1)-34 & 35,88 & 0,98 & 0,9 & 47 & 49 \\
\hline IV250(1)-35 & 36,88 & 0,98 & 0,9 & 47 & 49 \\
\hline IV250(1)-36 & 37,83 & 0,98 & 1,0 & 48 & 49 \\
\hline IV250(1)-37 & 42,94 & 0,98 & 1,0 & 48 & 50 \\
\hline IV250(1)-38 & 43,89 & 0,98 & 1,0 & 49 & 51 \\
\hline IV250(1)-39 & 44,90 & 0,95 & 1,0 & 49 & 41 \\
\hline
\end{tabular}

\begin{tabular}{|c|r|r|r|r|r|}
\hline & & & \multicolumn{1}{|c|}{$\mu \mathrm{mol} / 1$} \\
\hline Probe & Tage & g/h & pH & Al & \multicolumn{1}{c|}{ Si } \\
\hline \hline IV250(1)-41 & 46,87 & 0,97 & 1,0 & 50 & 51 \\
\hline IV250(1)-42 & 47,88 & 0,95 & 1,0 & 50 & 51 \\
\hline IV250(1)-43 & 48,84 & 1,00 & 1,0 & 50 & 51 \\
\hline IV250(1)-44 & 49,87 & 0,94 & 1,0 & 50 & 51 \\
\hline IV250(1)-45 & 50,87 & 0,96 & 1,0 & 51 & 52 \\
\hline IV250(1)-46 & 51,88 & 0,82 & 1,0 & 56 & 57 \\
\hline IV250(1)-47 & 52,88 & 0,94 & 1,0 & 50 & 51 \\
\hline IV250(1)-48 & 55,01 & 1,03 & 1,0 & 50 & 51 \\
\hline IV250(1)-49 & 56,87 & 1,01 & 1,0 & 50 & 52 \\
\hline IV250(1)-50 & 58,90 & 1,01 & 1,0 & 50 & 52 \\
\hline IV250(1)-51 & 69,92 & 0,94 & 1,1 & 50 & 51 \\
\hline \multicolumn{2}{|r|}{} & & & & \\
\hline Rate [mol/(g•h)] & & & $6,5 \bullet 10^{-8}$ & $6,6 \bullet 10^{-8}$ \\
\hline
\end{tabular}


Tab. A-14: Durchfluß-Experimente mit Kaolinit Versuch IV250(2).

\section{IV250(2)}

\section{Kaolinit}

$\varnothing=0,63-2 \mu \mathrm{m}$

Einwaage Mineral [g]: 0,7506

Einwaage Lösung [g]: 249,3

Lösung: $\mathrm{H}_{2} \mathrm{SO}_{4}, \mathbf{p H}=\mathbf{2}$

\begin{tabular}{|l|r|r|r|r|r|}
\hline & & & \multicolumn{1}{|l|}{$\mu$ mol/l } \\
\hline Probe & Tage & g/h & pH & Al & Si \\
\hline \hline IV250(2)-1 & 0,95 & 0,12 & 2,0 & 66 & 92 \\
\hline IV250(2)-2 & 1,91 & 0,07 & 1,9 & 69 & 96 \\
\hline IV250(2)-3 & 2,90 & 0,23 & 1,9 & 71 & 97 \\
\hline IV250(2)-4 & 4,63 & 0,37 & 2,0 & 70 & 96 \\
\hline IV250(2)-5 & 5,81 & 0,21 & 2,0 & 72 & 98 \\
\hline IV250(2)-6 & 7,03 & 0,23 & 2,0 & 72 & 97 \\
\hline IV250(2)-7 & 8,93 & 0,30 & 2,0 & 72 & 97 \\
\hline IV250(2)-8 & 10,77 & 0,38 & 2,0 & 72 & 96 \\
\hline IV250(2)-9 & 12,82 & 0,34 & 2,0 & 72 & 95 \\
\hline IV250(2)-10 & 14,09 & 0,30 & 2,0 & 71 & 94 \\
\hline IV250(2)-11 & 14,80 & 0,14 & 2,0 & 71 & 94 \\
\hline IV250(2)-12 & 16,03 & 0,26 & 2,0 & 71 & 93 \\
\hline IV250(2)-13 & 16,94 & 0,20 & 2,0 & 71 & 93 \\
\hline IV250(2)-14 & 17,99 & 0,24 & 2,0 & 70 & 91 \\
\hline IV250(2)-15 & 19,61 & 0,31 & 2,0 & 69 & 90 \\
\hline IV250(2)-16 & 21,75 & 0,35 & 2,0 & 70 & 91 \\
\hline IV250(2)-17 & 23,61 & 0,38 & 2,0 & 69 & 89 \\
\hline IV250(2)-18 & 25,64 & 0,34 & 2,0 & 71 & 91 \\
\hline IV250(2)-19 & 26,96 & 0,55 & 2,0 & 78 & 99 \\
\hline IV250(2)-20 & 28,72 & 0,51 & 2,0 & 80 & 100 \\
\hline IV250(2)-21 & 29,99 & 0,55 & 2,0 & 78 & 98 \\
\hline IV250(2)-22 & 30,92 & 0,54 & 2,0 & 76 & 95 \\
\hline IV250(2)-23 & 34,77 & 0,21 & 2,0 & 77 & 97 \\
\hline IV250(2)-24 & 36,87 & 0,41 & 2,0 & 76 & 95 \\
\hline IV250(2)-25 & 38,78 & 0,43 & 2,0 & 73 & 91 \\
\hline IV250(2)-26 & 40,82 & 0,45 & 2,0 & 70 & 88 \\
\hline IV250(2)-27 & 42,74 & 0,44 & 2,0 & 68 & 85 \\
\hline IV250(2)-28 & 44,65 & 0,46 & 2,0 & 67 & 83 \\
\hline IV250(2)-29 & 46,73 & 0,48 & 2,0 & 64 & 80 \\
\hline IV250(2)-30 & 48,77 & 0,48 & 2,0 & 59 & 76 \\
\hline IV250(2)-31 & 50,68 & 0,53 & 2,0 & 57 & 73 \\
\hline IV250(2)-32 & 52,74 & 0,48 & 2,0 & 47 & 60 \\
\hline IV250(2)-33 & 54,65 & 0,48 & 2,0 & 48 & 61 \\
\hline IV250(2)-34 & 56,76 & 0,43 & 2,0 & 48 & 61 \\
\hline IV250(2)-35 & 58,66 & 0,37 & 2,0 & 48 & 60 \\
\hline IV250(2)-36 & 60,67 & 0,38 & 2,0 & 48 & 60 \\
\hline IV250(2)-37 & 61,88 & 0,34 & 2,0 & 48 & 60 \\
\hline IV250(2)-38 & 72,73 & 0,38 & 2,0 & 47 & 59 \\
\hline
\end{tabular}

\begin{tabular}{|c|r|r|r|r|r|}
\hline & & & \multicolumn{3}{|c|}{$\mu \mathrm{mol} / 1$} \\
\hline Probe & Tage & g/h & pH & \multicolumn{1}{c|}{ Al } & \multicolumn{1}{c|}{ Si } \\
\hline \hline IV250(2)-41 & 95,68 & 0,45 & 2,1 & 41 & 52 \\
\hline IV250(2)-42 & 97,83 & 0,41 & 2,1 & 42 & 52 \\
\hline IV250(2)-43 & 99,76 & 0,42 & 2,1 & 41 & 51 \\
\hline IV250(2)-44 & 101,70 & 0,38 & 2,1 & 40 & 50 \\
\hline IV250(2)-45 & 103,84 & 0,37 & 2,0 & 41 & 51 \\
\hline IV250(2)-46 & 105,67 & 0,41 & 2,0 & 41 & 50 \\
\hline IV250(2)-47 & 107,74 & 0,43 & 2,0 & 41 & 50 \\
\hline IV250(2)-48 & 109,82 & 0,44 & 2,0 & 41 & 50 \\
\hline IV250(2)-49 & 111,85 & 0,38 & 2,0 & 41 & 50 \\
\hline IV250(2)-50 & 113,86 & 0,34 & 2,0 & 41 & 50 \\
\hline IV250(2)-51 & 115,95 & 0,35 & 2,1 & 41 & 50 \\
\hline IV250(2)-52 & 117,84 & 0,36 & 2,1 & 42 & 50 \\
\hline IV250(2)-53 & 120,05 & 0,42 & 2,1 & 43 & 50 \\
\hline IV250(2)-54 & 122,02 & 0,34 & 2,1 & 42 & 49 \\
\hline IV250(2)-55 & 124,03 & 0,32 & 2,1 & 42 & 49 \\
\hline IV250(2)-56 & 126,03 & 0,35 & 2,1 & 42 & 49 \\
\hline IV250(2)-57 & 127,98 & 0,36 & 2,1 & 42 & 49 \\
\hline IV250(2)-58 & 130,07 & 0,38 & 2,1 & 42 & 49 \\
\hline IV250(2)-59 & 131,99 & 0,39 & 2,0 & 40 & 48 \\
\hline IV250(2)-60 & 133,84 & 0,15 & 2,0 & 39 & 48 \\
\hline IV250(2)-61 & 135,96 & 0,36 & 2,0 & 39 & 47 \\
\hline & & & & & \\
\hline Rate [mol/(g•h)] & & & $1,9 \bullet 10^{-8}$ & $2,2 \bullet 10^{-8}$ \\
\hline
\end{tabular}


Tab. A-15: Durchfluß-Experimente mit Kaolinit Versuch IV250(4).

IV250(4)

Kaolinit

$\varnothing=0,63-2 \mu \mathrm{m}$

Einwaage Mineral [g]: 0,7506

Einwaage Lösung [g]: 249,3

Lösung: $\mathrm{H}_{2} \mathrm{SO}_{4}, \mathbf{p H}=\mathbf{2}$

\begin{tabular}{|c|c|c|c|c|c|}
\hline & & & & \multicolumn{2}{|l|}{$\mu \mathrm{mol} / 1$} \\
\hline Probe & Tage & $\mathrm{g} / \mathrm{h}$ & pH & $\mathbf{A l}$ & $\mathbf{S i}$ \\
\hline$\overline{\text { IV250(4)-1 }}$ & $\overline{7,63}$ & 1,18 & $\overline{2,0}$ & 46 & $\overline{70}$ \\
\hline IV250(4)-2 & 12,62 & 1,16 & 2,0 & 11 & 28 \\
\hline IV250(4)-3 & 19,69 & 1,19 & 2,0 & 11 & 29 \\
\hline IV250(4)-4 & 26,65 & 1,18 & 1,9 & 11 & 27 \\
\hline IV250( & 28,67 & 1,16 & $\overline{1,9}$ & 11 & 26 \\
\hline 250 & 33,69 & 1,18 & 1,9 & 10 & 26 \\
\hline IV250(4)-7 & 40,68 & 1,18 & $\overline{2,0}$ & 11 & 25 \\
\hline IV250(4)-8 & 47,67 & 1,20 & 1,9 & 11 & 25 \\
\hline$\sqrt{250(4)-9}$ & 78,67 & 1,13 & 2,1 & 13 & 28 \\
\hline IV250(4)-10 & 83,66 & 1,24 & 2,0 & 13 & 28 \\
\hline IV250(4)-11 & 91,72 & 1,15 & 2,0 & 13 & 29 \\
\hline IV250(4)-12 & 97,68 & 1,17 & 2,0 & 13 & 27 \\
\hline IV250(4)-13 & 103,67 & 1,17 & 2,0 & 14 & 26 \\
\hline IV250(4)-14 & 112,79 & 1,15 & 2,1 & 11 & 28 \\
\hline IV250(4)-15 & 121,78 & 1,19 & 2,0 & 12 & 27 \\
\hline IV250(4)-16 & 125,78 & 1,12 & 2,0 & 12 & 27 \\
\hline IV250(4)-17 & 131,80 & 1,00 & 2,0 & 13 & 30 \\
\hline IV250 & 138,67 & 1,18 & 2,0 & 13 & 28 \\
\hline IV250(4)-19 & 145,72 & 1,17 & 2,0 & 12 & 27 \\
\hline IV250(4)-20 & 153,64 & 1,03 & 2,0 & 14 & 30 \\
\hline IV250(4)-21 & 159,67 & 1,18 & 2,0 & 13 & 27 \\
\hline IV250(4)-22 & 166,61 & 1,22 & 2,0 & 12 & 27 \\
\hline & & & & $9 \cdot 10^{-8}$ & $4,2 \cdot 10^{\circ}$ \\
\hline
\end{tabular}


Tab. A-16: Durchfluß-Experimente mit Kaolinit Versuch V90.

\section{V90 \\ Kaolinit \\ $\varnothing=0,63-2 \mu \mathrm{m}$ \\ Einwaage Mineral [g]: 0,2702 \\ Einwaage Lösung [g]: 88,8 \\ Lösung: $\mathrm{HNO}_{3}, \mathbf{p H}=1$}

\begin{tabular}{|c|c|c|c|c|c|}
\hline & & & & $\mu \mathrm{mol} / 1$ & \\
\hline Probe & Tage & $\mathrm{g} / \mathrm{h}$ & $\mathbf{p H}$ & Al & $\mathbf{S i}$ \\
\hline V90-1 & 0,97 & 0,58 & 1,2 & 3,9 & $\overline{9,1}$ \\
\hline V90-2 & 1,98 & 0,57 & 1,3 & 6,5 & 15 \\
\hline V90-3 & 3,00 & 0,54 & 1,5 & 7,3 & 19 \\
\hline V90-4 & 3,95 & 0,56 & 1,4 & 8,4 & 21 \\
\hline V90-5 & 6,13 & 0,45 & 1,4 & 9,8 & 24 \\
\hline V90-6 & 7,09 & 0,56 & 1,3 & 11 & 25 \\
\hline V90-7 & 7,93 & 0,55 & 1,2 & 11 & 26 \\
\hline V90-8 & 8,88 & 0,51 & 1,3 & 11 & 26 \\
\hline V90-9 & 10,02 & 0,51 & 1,3 & 12 & 27 \\
\hline V90-10 & 10,88 & 0,51 & 1,4 & 12 & 29 \\
\hline V90-11 & 12,00 & 0,52 & 1,3 & 13 & 29 \\
\hline V90-12 & 12,84 & 0,51 & 1,3 & 14 & 31 \\
\hline V90-13 & 13,93 & 0,51 & 1,3 & 15 & 32 \\
\hline V90-14 & 14,92 & 0,50 & 1,3 & 15 & 32 \\
\hline V90-15 & 16,03 & 0,41 & 1,3 & 16 & 34 \\
\hline V90-16 & 17,08 & 0,39 & 1,3 & 16 & 35 \\
\hline V90-17 & 18,01 & 0,34 & 1,4 & 16 & 31 \\
\hline V90-18 & 20,87 & 0,15 & 1,3 & 21 & 40 \\
\hline V90-19 & 23,99 & 0,12 & 1,2 & 24 & 46 \\
\hline V90-20 & 25,90 & 0,23 & 1,2 & 24 & 46 \\
\hline V90-21 & 27,97 & 0,26 & 1,2 & 27 & 50 \\
\hline V90-22 & 29,01 & 0,31 & 1,2 & 30 & 55 \\
\hline V90-23 & 29,94 & 0,93 & 1,2 & 29 & 51 \\
\hline V90-24 & 30,98 & 0,43 & 1,1 & 28 & 48 \\
\hline V90-25 & 32,95 & 0,35 & 1,1 & 29 & 49 \\
\hline V90-26 & 33,98 & 0,30 & 1,1 & 32 & 52 \\
\hline V90-27 & 34,93 & 0,28 & 1,1 & 32 & 53 \\
\hline V90-28 & 35,92 & 0,45 & 1,1 & 30 & 52 \\
\hline V90-29 & 37,65 & 0,48 & 1,1 & 29 & 49 \\
\hline V90-30 & 38,83 & 0,39 & 1,1 & 29 & 48 \\
\hline V90-31 & 40,06 & 0,41 & 1,1 & 30 & 48 \\
\hline V90-32 & 41,95 & 0,42 & 1,1 & 30 & 48 \\
\hline V90-33 & 43,79 & 0,48 & 1,1 & 29 & 47 \\
\hline V90-34 & 45,84 & 0,42 & 1,1 & 29 & 46 \\
\hline V90-35 & 47,11 & 0,46 & 1,1 & 28 & 45 \\
\hline V90-36 & 47,82 & 0,48 & 1,1 & 27 & 45 \\
\hline V90-37 & 49,05 & 0,46 & 1,1 & 27 & 44 \\
\hline V90-38 & 49,96 & 0,46 & 1,1 & 26 & 43 \\
\hline V90-39 & 51,01 & 0,48 & 1,1 & 25 & 43 \\
\hline V90-40 & 52,63 & 0,45 & 1,1 & 24 & 42 \\
\hline V90-41 & 54,77 & 0,45 & 1,1 & 22 & 40 \\
\hline V90-42 & 56,63 & 0,48 & 1,2 & 23 & 41 \\
\hline
\end{tabular}

\begin{tabular}{|c|c|c|c|c|c|}
\hline & & & & $\mu \mathrm{mol} / \mathrm{l}$ & \\
\hline Probe & Tage & $\mathrm{g} / \mathbf{h}$ & pH & Al & Si \\
\hline V90-43 & |58,66 & 0,50 & $\overline{1,3}$ & 23 & 41 \\
\hline V90-44 & 59,98 & 0,53 & 1,3 & 22 & 39 \\
\hline V90-45 & 61,74 & 0,52 & 1,3 & 21 & 39 \\
\hline V90-46 & 63,01 & 0,53 & 1,3 & 22 & 41 \\
\hline V90-47 & 63,94 & 0,52 & 1,3 & 21 & 40 \\
\hline V90-48 & 69,89 & 0,40 & 1,2 & 25 & 49 \\
\hline V90-49 & 71,81 & 0,43 & 1,2 & 24 & 46 \\
\hline V90-50 & 73,84 & 0,43 & 1,2 & 23 & 44 \\
\hline V90-51 & 75,76 & 0,43 & 1,2 & 22 & 44 \\
\hline V90-52 & 77,67 & 0,45 & 1,2 & 21 & 44 \\
\hline V90-53 & 79,75 & 0,49 & 1,2 & 21 & 42 \\
\hline V90-54 & 81,79 & 0,48 & 1,2 & 20 & 40 \\
\hline V90-55 & 83,70 & 0,50 & 1,3 & 19 & 38 \\
\hline V90-56 & 85,76 & 0,48 & 1,3 & 20 & 38 \\
\hline V90-57 & 87,67 & 0,48 & 1,3 & 22 & 41 \\
\hline V90-58 & 89,78 & 0,44 & 1,3 & 23 & 47 \\
\hline V90-59 & 91,68 & 0,37 & 1,3 & 25 & 50 \\
\hline V90-60 & 93,69 & 0,39 & 1,2 & 25 & 53 \\
\hline V90-61 & 94,90 & 0,34 & 1,2 & 25 & 55 \\
\hline V90-62 & 105,75 & 0,38 & 1,2 & 23 & 62 \\
\hline V90-63 & 126,93 & 0,43 & 1,2 & 18 & 51 \\
\hline V90-64 & 128,70 & 0,45 & 1,3 & 16 & 43 \\
\hline V90-65 & 130,85 & 0,42 & 1,3 & 16 & 43 \\
\hline V90-66 & 132,78 & 0,41 & 1,3 & 16 & 44 \\
\hline V90-67 & 134,73 & 0,37 & 1,3 & 16 & 40 \\
\hline V90-68 & 136,86 & 0,37 & 1,3 & 18 & 45 \\
\hline V90-69 & 138,69 & 0,41 & 1,3 & 20 & 48 \\
\hline V90-70 & 140,76 & 0,42 & 1,1 & 20 & 50 \\
\hline V90-71 & 142,84 & 0,43 & 1,2 & 20 & 49 \\
\hline V90-72 & 144,88 & 0,38 & 1,2 & 20 & 47 \\
\hline V90-73 & 146,88 & 0,33 & 1,2 & 19 & 46 \\
\hline V90-74 & 148,98 & 0,35 & 1,3 & 19 & 45 \\
\hline V90-75 & 150,86 & 0,36 & 1,3 & 19 & 44 \\
\hline V90-76 & 153,07 & 0,42 & 1,4 & 18 & 43 \\
\hline V90-77 & 155,04 & 0,34 & 1,3 & 17 & 41 \\
\hline V90-78 & 157,06 & 0,33 & 1,3 & 17 & 42 \\
\hline V90-79 & 159,06 & 0,35 & 1,3 & 17 & 42 \\
\hline V90-80 & 161,00 & 0,36 & 1,3 & 16 & 41 \\
\hline V90-81 & 163,09 & 0,37 & 1,3 & 17 & 42 \\
\hline V90-82 & 165,01 & 0,37 & 1,3 & 17 & 36 \\
\hline V90-83 & 166,86 & 0,39 & 1,3 & 16 & 41 \\
\hline V90-84 & 168,98 & 0,36 & 1,3 & 16 & 40 \\
\hline \multicolumn{2}{|c|}{ Rate $[\mathrm{mol} /(\mathrm{g} \bullet \mathrm{h})]$} & & & $2,3 \cdot 10^{-8}$ & $5,6 \cdot 10^{-8}$ \\
\hline
\end{tabular}


Tab. A-17: Durchfluß-Experimente mit Kaolinit Versuch V250(2).

V250(2)

\section{Kaolinit}

$\varnothing=0,63-2 \mu \mathrm{m}$

Einwaage Mineral [g]: 0,7504

Einwaage Lösung [g]: 249,5

Lösung: $\mathrm{HNO}_{3}, \mathbf{p H}=1$

\begin{tabular}{|c|r|r|r|r|r|}
\hline & & & & \multicolumn{1}{|c|}{$\mu$ mol/l } \\
\hline Probe & \multicolumn{1}{|c|}{ Tage } & g/h & pH & \multicolumn{1}{|c|}{ Al } & \multicolumn{1}{|c|}{ Si } \\
\hline \hline V250(2)-1 & 1,09 & 1,03 & 1,2 & 139 & 179 \\
\hline V250(2)-2 & 2,81 & 0,42 & 1,2 & 131 & 171 \\
\hline V250(2)-3 & 4,98 & 0,46 & 1,2 & 128 & 167 \\
\hline V250(2)-4 & 6,69 & 0,46 & 1,2 & 124 & 162 \\
\hline V250(2)-5 & 6,99 & 0,47 & 1,2 & 122 & 159 \\
\hline V250(2)-6 & 7,73 & 0,46 & 1,3 & 122 & 159 \\
\hline V250(2)-7 & 8,09 & 0,46 & 1,3 & 120 & 157 \\
\hline V250(2)-8 & 8,73 & 0,47 & 1,2 & 119 & 155 \\
\hline V250(2)-9 & 13,09 & 0,44 & 1,2 & 114 & 149 \\
\hline V250(2)-10 & 13,79 & 0,45 & 1,3 & 112 & 146 \\
\hline V250(2)-11 & 15,78 & 0,47 & 1,3 & 108 & 140 \\
\hline V250(2)-12 & 20,75 & 0,47 & 1,1 & 106 & 128 \\
\hline V250(2)-13 & 23,74 & 0,48 & 1,1 & 104 & 126 \\
\hline V250(2)-14 & 27,79 & 0,46 & 1,1 & 98 & 123 \\
\hline V250(2)-15 & 37,10 & 0,64 & 1,1 & 100 & 126 \\
\hline V250(2)-16 & 43,00 & 0,68 & 1,1 & 82 & 101 \\
\hline V250(2)-17 & 55,10 & 0,95 & 1,1 & 69 & 84 \\
\hline V250(2)-18 & 62,10 & 1,03 & 1,1 & 65 & 79 \\
\hline V250(2)-19 & 68,95 & 1,01 & 1,1 & 61 & 74 \\
\hline V250(2)-20 & 75,74 & 1,01 & 1,1 & 62 & 76 \\
\hline V250(2)-21 & 77,00 & 0,80 & 1,1 & 62 & 76 \\
\hline V250(2)-22 & 80,16 & 0,81 & 1,2 & 66 & 88 \\
\hline V250(2)-23 & 83,09 & 0,85 & 1,2 & 67 & 89 \\
\hline V250(2)-24 & 87,12 & 0,83 & 1,1 & 67 & 89 \\
\hline V250(2)-25 & 90,96 & 0,81 & 1,1 & 71 & 94 \\
\hline V250(2)-26 & 94,24 & 0,82 & 1,1 & 69 & 92 \\
\hline V250(2)-27 & 97,04 & 0,85 & 1,1 & 70 & 92 \\
\hline V250(2)-28 & 101,13 & 0,80 & 1,1 & 69 & 90 \\
\hline V250(2)-29 & 104,13 & 0,86 & 1,1 & 66 & 87 \\
\hline V250(2)-30 & 112,07 & 0,91 & 1,1 & 85 & 94 \\
\hline V250(2)-31 & 171,00 & 0,84 & 1,0 & 58 & 83 \\
\hline V250(2)-32 & 173,89 & 1,28 & 1,0 & 48 & 70 \\
\hline V250(2)-33 & 175,99 & 0,82 & 1,0 & 42 & 60 \\
\hline V250(2)-34 & 178,00 & 0,90 & 1,0 & 38 & 56 \\
\hline V250(2)-35 & 181,10 & 0,93 & 1,0 & 33 & 50 \\
\hline V250(2)-36 & 187,92 & 0,94 & 1,1 & 13 & 23 \\
\hline V250(2)-37 & 189,95 & 0,88 & 1,0 & 26 & 39 \\
\hline V250(2)-38 & 190,97 & 0,92 & 1,0 & 25 & 38 \\
\hline V250(2)-39 & 196,98 & 0,90 & 1,0 & 24 & 37 \\
\hline V250(2)-40 & 198,91 & 1,06 & 1,0 & 23 & 37 \\
\hline
\end{tabular}

\begin{tabular}{|c|c|r|r|r|r|}
\hline & & & \multicolumn{1}{|c|}{$\mu \mathrm{mol} / 1$} \\
\hline Probe & Tage & g/h & pH & \multicolumn{1}{c|}{ Al } & \multicolumn{1}{c|}{$\mathbf{~ S i}$} \\
\hline \hline V250(2)-41 & 203,15 & 0,95 & 1,1 & 22 & 35 \\
\hline V250(2)-42 & 205,06 & 0,93 & 1,1 & 22 & 35 \\
\hline V250(2)-43 & 208,96 & 1,02 & 1,1 & 22 & 36 \\
\hline V250(2)-44 & 210,97 & 0,98 & 1,1 & 22 & 33 \\
\hline V250(2)-45 & 216,06 & 0,96 & 1,1 & 21 & 32 \\
\hline V250(2)-46 & 218,00 & 0,98 & 1,0 & 23 & 39 \\
\hline V250(2)-47 & 220,03 & 0,88 & 1,0 & 22 & 38 \\
\hline V250(2)-48 & 225,03 & 0,80 & 1,0 & 20 & 37 \\
\hline V250(2)-49 & 226,92 & 0,89 & 1,0 & 20 & 38 \\
\hline V250(2)-50 & 230,01 & 0,89 & 1,0 & 21 & 37 \\
\hline V250(2)-51 & 232,08 & 1,30 & 1,0 & 21 & 39 \\
\hline V250(2)-52 & 233,32 & 0,81 & 1,0 & 20 & 33 \\
\hline V250(2)-53 & 237,93 & 0,80 & 1,0 & 28 & 43 \\
\hline V250(2)-54 & 240,07 & 0,93 & 1,0 & 23 & 41 \\
\hline V250(2)-55 & 244,04 & 0,83 & 1,0 & 27 & 49 \\
\hline V250(2)-56 & 246,98 & 0,94 & 1,0 & 11 & 18 \\
\hline V250(2)-57 & 251,02 & 1,06 & 1,0 & 21 & 41 \\
\hline V250(2)-58 & 253,06 & 0,97 & 1,0 & 22 & 42 \\
\hline V250(2)-59 & 254,96 & 0,95 & 1,0 & 21 & 36 \\
\hline V250(2)-60 & 258,95 & 0,97 & 1,1 & 22 & 44 \\
\hline V250(2)-61 & 262,06 & 1,04 & 1,0 & 21 & 40 \\
\hline V250(2)-62 & 265,03 & 0,80 & 1,0 & 21 & 41 \\
\hline & & & & & \\
\hline Rate [mol/(g•h)] & & & $2,7 \bullet 10^{-8}$ & $4,9 \bullet 10^{-8}$ \\
\hline
\end{tabular}


Tab. A-18: Durchfluß-Experimente mit Kaolinit Versuch VI90.

\section{VI90}

\section{Kaolinit}

$\varnothing=0,63-2 \mu \mathrm{m}$

Einwaage Mineral [g]: 0,2702

Einwaage Lösung [g]: 87,8

Lösung: HCl, pH = 1

\begin{tabular}{|l|r|r|r|r|r|}
\hline \multicolumn{1}{|c|}{ Probe } & Tage & g/h & pH & Al & \multicolumn{1}{|c|}{ Si } \\
\hline \hline VI90-1 & 0,97 & 0,65 & 1,1 & 5,7 & 9,5 \\
\hline VI90-2 & 1,98 & 0,63 & 1,2 & 7,4 & 14 \\
\hline VI90-3 & 3,00 & 0,88 & 1,2 & 7,5 & 16 \\
\hline VI90-4 & 3,95 & 0,71 & 1,2 & 7,8 & 16 \\
\hline VI90-5 & 6,13 & 0,45 & 1,2 & 7,4 & 14 \\
\hline VI90-6 & 7,09 & 0,98 & 1,2 & 7,1 & 13 \\
\hline VI90-7 & 7,93 & 1,11 & 1,2 & 5,9 & 11 \\
\hline VI90-8 & 8,88 & 0,50 & 1,2 & 7,0 & 12 \\
\hline VI90-9 & 10,02 & 0,51 & 1,2 & 7,6 & 13 \\
\hline VI90-10 & 10,88 & 0,51 & 1,2 & 8,5 & 15 \\
\hline VI90-11 & 12,00 & 0,51 & 1,2 & 8,7 & 15 \\
\hline VI90-12 & 12,84 & 0,50 & 1,2 & 9,1 & 16 \\
\hline VI90-13 & 13,93 & 0,50 & 1,2 & 9,4 & 17 \\
\hline VI90-14 & 14,92 & 0,50 & 1,2 & 9,8 & 18 \\
\hline VI90-15 & 16,03 & 0,42 & 1,2 & 11 & 19 \\
\hline VI90-16 & 17,08 & 0,40 & 1,2 & 11 & 20 \\
\hline VI90-17 & 18,01 & 0,31 & 1,2 & 12 & 20 \\
\hline VI90-18 & 20,87 & 0,14 & 1,2 & 13 & 22 \\
\hline VI90-19 & 23,99 & 0,12 & 1,1 & 17 & 27 \\
\hline VI90-20 & 25,90 & 0,25 & 1,1 & 16 & 26 \\
\hline VI90-21 & 27,97 & 0,26 & 1,1 & 20 & 31 \\
\hline VI90-22 & 29,01 & 0,52 & 1,1 & 22 & 33 \\
\hline VI90-23 & 29,94 & 0,79 & 1,1 & 21 & 31 \\
\hline VI90-24 & 30,98 & 0,83 & 1,1 & 21 & 30 \\
\hline VI90-25 & 32,95 & 0,38 & 1,1 & 20 & 27 \\
\hline VI90-26 & 33,98 & 0,31 & 1,1 & 26 & 33 \\
\hline VI90-27 & 34,93 & 0,28 & 1,1 & 26 & 35 \\
\hline VI90-28 & 35,92 & 0,46 & 1,1 & 25 & 34 \\
\hline VI90-29 & 37,65 & 0,50 & 1,1 & 24 & 32 \\
\hline VI90-30 & 38,83 & 0,39 & 1,1 & 24 & 32 \\
\hline VI90-31 & 40,06 & 0,40 & 1,1 & 24 & 32 \\
\hline VI90-32 & 41,95 & 0,43 & 1,1 & 23 & 31 \\
\hline VI90-33 & 43,79 & 0,49 & 1,1 & 22 & 29 \\
\hline VI90-34 & 45,84 & 0,46 & 1,1 & 21 & 28 \\
\hline VI90-35 & 47,11 & 0,47 & 1,1 & 20 & 27 \\
\hline VI90-36 & 47,82 & 0,48 & 1,1 & 20 & 26 \\
\hline VI90-37 & 49,05 & 0,47 & 1,1 & 19 & 26 \\
\hline VI90-38 & 49,96 & 0,47 & 1,1 & 19 & 25 \\
\hline VI90-39 & 51,01 & 0,48 & 1,1 & 18 & 25 \\
\hline VI90-40 & 52,63 & 0,46 & 1,1 & 17 & 24 \\
\hline VI90-41 & 54,77 & 0,45 & 1,1 & 16 & 23 \\
\hline
\end{tabular}

\begin{tabular}{|c|c|c|c|c|c|}
\hline & & & & $\mu \mathrm{mol} / 1$ & \\
\hline Probe & Tage & g/h & pH & $\mathrm{Al}$ & $\mathbf{S i}$ \\
\hline VI90-43 & 58,66 & 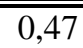 & 1,3 & 14 & 22 \\
\hline VI90-44 & 59,98 & 0,54 & 1,3 & 14 & 21 \\
\hline VI90-45 & 61,74 & 0,53 & 1,3 & 13 & 20 \\
\hline VI90-46 & 63,01 & 0,53 & 1,3 & 13 & 20 \\
\hline VI90-47 & 63,94 & 0,52 & 1,3 & 13 & 19 \\
\hline VI90-48 & 67,80 & 0,21 & 1,3 & 15 & 22 \\
\hline VI90-49 & 69,89 & 0,40 & 1,3 & 17 & 25 \\
\hline VI90-50 & 71,81 & 0,42 & 1,3 & 17 & 25 \\
\hline VI90-51 & 73,84 & 0,43 & 1,3 & 17 & 25 \\
\hline VI90-52 & 75,76 & 0,45 & 1,3 & 18 & 25 \\
\hline VI90-53 & 77,67 & 0,46 & 1,3 & 19 & 24 \\
\hline VI90-54 & 79,75 & 0,48 & 1,3 & 19 & 24 \\
\hline VI90-55 & 81,79 & 0,48 & 1,3 & 19 & 24 \\
\hline VI90-56 & 83,70 & 0,51 & 1,3 & 18 & 24 \\
\hline VI90-57 & 85,76 & 0,48 & 1,3 & 17 & 21 \\
\hline VI90-58 & 87,67 & 0,51 & 1,2 & 17 & 22 \\
\hline VI90-59 & 89,78 & 0,44 & 1,2 & 18 & 23 \\
\hline VI90-60 & 91,68 & 0,37 & 1,2 & 19 & 25 \\
\hline VI90-61 & 93,69 & 0,39 & 1,2 & 19 & 26 \\
\hline VI90-62 & 94,90 & 0,35 & 1,2 & 19 & 27 \\
\hline VI90-63 & 105,75 & 0,38 & 1,2 & 20 & 29 \\
\hline VI90-64 & 107,02 & 0,42 & 1,2 & 20 & 29 \\
\hline VI90-65 & 126,93 & 0,43 & 1,2 & 18 & 29 \\
\hline VI90-66 & 128,70 & 0,46 & 1,3 & 16 & 27 \\
\hline VI90-67 & 130,85 & 0,42 & 1,3 & 16 & 26 \\
\hline VI90-68 & 132,78 & 0,42 & 1,2 & 15 & 25 \\
\hline VI90-69 & 134,73 & 0,37 & 1,2 & 15 & 25 \\
\hline VI90-70 & 136,86 & 0,36 & 1,2 & 15 & 25 \\
\hline VI90-71 & 138,69 & 0,41 & 1,2 & 15 & 25 \\
\hline VI90-72 & 140,76 & 0,42 & 1,2 & 16 & 26 \\
\hline VI90-73 & 142,84 & 0,43 & 1,2 & 15 & 25 \\
\hline VI90-74 & 144,88 & 0,38 & 1,2 & 15 & 24 \\
\hline VI90-75 & 146,88 & 0,33 & 1,2 & 16 & 24 \\
\hline VI90-76 & 148,98 & 0,35 & 1,3 & 15 & 23 \\
\hline VI90-77 & 150,86 & 0,36 & 1,3 & 15 & 24 \\
\hline VI90-78 & 153,07 & 0,41 & 1,3 & 15 & 22 \\
\hline VI90-79 & 155,04 & 0,33 & 1,3 & 14 & 22 \\
\hline VI90-80 & 157,06 & 0,32 & 1,3 & 15 & 21 \\
\hline VI90-81 & 159,06 & 0,35 & 1,3 & 15 & 22 \\
\hline VI90-82 & 161,00 & 0,36 & 1,3 & 16 & 23 \\
\hline VI90-83 & 163,09 & 0,37 & 1,3 & 17 & 24 \\
\hline VI90-84 & 165,01 & 0,39 & 1,3 & 16 & 26 \\
\hline VI90-85 & 166,86 & 0,36 & 1,3 & 17 & 26 \\
\hline VI90-86 & 168,98 & 0,35 & 1,3 & 16 & 24 \\
\hline \multicolumn{2}{|c|}{ Rate $[\mathrm{mol} /(\mathrm{g} \bullet \mathrm{h})]$} & & & $2,1 \cdot 10^{-8}$ & $3,1 \cdot 10^{-8}$ \\
\hline
\end{tabular}


Tab. A-19: Durchfluß-Experimente mit Kaolinit Versuch VI250(1).

\section{VI250(1)}

\section{Kaolinit}

$\emptyset=0,63-2 \mu \mathrm{m}$

Einwaage Mineral [g]: 0,7508

Einwaage Lösung [g]: 248,9

Lösung: HCl, pH = 1

\begin{tabular}{|c|r|r|r|r|r|}
\hline & & & \multicolumn{1}{|c|}{$\boldsymbol{\mu m o l} / \mathbf{l}$} \\
\hline Probe & Tage & g/h & pH & Al & \multicolumn{1}{c|}{ Si } \\
\hline \hline VI250(1)-1 & 0,97 & 0,86 & 1,1 & 5,6 & 9,5 \\
\hline VI250(1)-2 & 1,98 & 0,71 & 1,3 & 8,0 & 15 \\
\hline VI250(1)-3 & 3,00 & 0,70 & 1,2 & 8,8 & 20 \\
\hline VI250(1)-4 & 3,95 & 0,67 & 1,2 & 10 & 23 \\
\hline VI250(1)-5 & 6,13 & 0,46 & 1,2 & 12 & 26 \\
\hline VI250(1)-6 & 7,09 & 0,73 & 1,1 & 13 & 28 \\
\hline VI250(1)-7 & 7,93 & 0,75 & 1,2 & 13 & 29 \\
\hline VI250(1)-8 & 8,88 & 0,50 & 1,1 & 14 & 30 \\
\hline VI250(1)-9 & 10,02 & 0,51 & 1,2 & 15 & 32 \\
\hline VI250(1)-10 & 10,88 & 0,51 & 1,1 & 17 & 35 \\
\hline VI250(1)-11 & 12,00 & 0,51 & 1,2 & 18 & 37 \\
\hline VI250(1)-12 & 12,84 & 0,50 & 1,2 & 20 & 40 \\
\hline VI250(1)-13 & 13,93 & 0,50 & 1,2 & 21 & 43 \\
\hline VI250(1)-14 & 16,03 & 0,43 & 1,1 & 26 & 50 \\
\hline VI250(1)-15 & 17,08 & 0,40 & 1,2 & 27 & 52 \\
\hline VI250(1)-16 & 18,01 & 0,32 & 1,1 & 30 & 52 \\
\hline VI250(1)-17 & 20,87 & 0,14 & 1,1 & 33 & 57 \\
\hline VI250(1)-18 & 23,99 & 0,12 & 1,1 & 39 & 66 \\
\hline VI250(1)-19 & 25,90 & 0,25 & 1,1 & 44 & 73 \\
\hline VI250(1)-20 & 27,97 & 0,27 & 1,1 & 47 & 78 \\
\hline VI250(1)-21 & 29,01 & 0,46 & 1,1 & 52 & 84 \\
\hline VI250(1)-22 & 29,94 & 0,74 & 1,2 & 52 & 81 \\
\hline VI250(1)-23 & 30,98 & 0,84 & 1,1 & 54 & 84 \\
\hline VI250(1)-24 & 32,95 & 0,38 & 1,2 & 49 & 75 \\
\hline VI250(1)-25 & 33,98 & 0,31 & 1,2 & 55 & 81 \\
\hline VI250(1)-26 & 34,93 & 0,28 & 1,1 & 57 & 83 \\
\hline VI250(1)-27 & 35,92 & 0,45 & 1,1 & 58 & 85 \\
\hline VI250(1)-28 & 37,65 & 0,48 & 1,1 & 58 & 85 \\
\hline VI250(1)-29 & 38,83 & 0,38 & 1,1 & 61 & 87 \\
\hline VI250(1)-30 & 40,06 & 0,39 & 1,1 & 63 & 90 \\
\hline VI250(1)-31 & 41,95 & 0,43 & 1,1 & 63 & 90 \\
\hline VI250(1)-32 & 43,79 & 0,48 & 1,1 & 66 & 94 \\
\hline VI250(1)-33 & 45,84 & 0,42 & 1,1 & 67 & 93 \\
\hline VI250(1)-34 & 47,11 & 0,36 & 1,1 & 69 & 96 \\
\hline VI250(1)-35 & 47,82 & 0,48 & 1,1 & 70 & 96 \\
\hline VI250(1)-36 & 49,05 & 0,47 & 1,1 & 71 & 97 \\
\hline VI250(1)-37 & 49,96 & 0,47 & 1,1 & 73 & 100 \\
\hline VI250(1)-38 & 51,01 & 0,48 & 1,1 & 73 & 101 \\
\hline VI250(1)-39 & 52,63 & 0,46 & 1,1 & 74 & 102 \\
\hline
\end{tabular}

\begin{tabular}{|l|r|r|r|r|r|}
\hline & & & \multicolumn{1}{|c|}{$\boldsymbol{\mu m o l} / 1$} \\
\hline Probe & Tage & g/h & pH & \multicolumn{1}{|c|}{ Al } & \multicolumn{1}{c|}{ Si } \\
\hline \hline VI250(1)-43 & 59,98 & 0,53 & 1,3 & 79 & 110 \\
\hline VI250(1)-44 & 61,74 & 0,52 & 1,3 & 80 & 111 \\
\hline VI250(1)-45 & 63,01 & 0,55 & 1,3 & 81 & 113 \\
\hline VI250(1)-46 & 63,94 & 0,99 & 1,3 & 92 & 127 \\
\hline VI250(1)-47 & 67,80 & 0,21 & 1,3 & 117 & 161 \\
\hline VI250(1)-48 & 69,89 & 0,39 & 1,3 & 113 & 156 \\
\hline VI250(1)-49 & 71,81 & 0,42 & 1,3 & 105 & 147 \\
\hline VI250(1)-50 & 73,84 & 0,44 & 1,3 & 100 & 140 \\
\hline VI250(1)-51 & 75,76 & 0,43 & 1,3 & 95 & 133 \\
\hline VI250(1)-52 & 77,67 & 0,45 & 1,3 & 92 & 127 \\
\hline VI250(1)-53 & 79,75 & 0,49 & 1,3 & 88 & 121 \\
\hline VI250(1)-54 & 81,79 & 0,48 & 1,2 & 79 & 113 \\
\hline VI250(1)-55 & 83,70 & 0,49 & 1,2 & 78 & 113 \\
\hline VI250(1)-56 & 85,76 & 0,49 & 1,2 & 62 & 88 \\
\hline VI250(1)-57 & 87,67 & 0,47 & 1,2 & 62 & 88 \\
\hline VI250(1)-58 & 89,78 & 0,42 & 1,2 & 62 & 88 \\
\hline VI250(1)-59 & 91,68 & 0,36 & 1,2 & 66 & 93 \\
\hline VI250(1)-60 & 93,69 & 0,39 & 1,2 & 63 & 90 \\
\hline VI250(1)-61 & 94,90 & 0,34 & 1,1 & 64 & 91 \\
\hline VI250(1)-62 & 105,75 & 0,38 & 1,2 & 64 & 93 \\
\hline VI250(1)-63 & 107,02 & 0,42 & 1,2 & 65 & 95 \\
\hline VI250(1)-64 & 126,93 & 0,43 & 1,2 & 65 & 98 \\
\hline VI250(1)-65 & 128,70 & 0,45 & 1,2 & 66 & 100 \\
\hline VI250(1)-66 & 130,85 & 0,40 & 1,1 & 69 & 104 \\
\hline VI250(1)-67 & 132,78 & 0,41 & 1,2 & 66 & 102 \\
\hline VI250(1)-68 & 134,73 & 0,37 & 1,2 & 66 & 101 \\
\hline VI250(1)-69 & 136,86 & 0,37 & 1,2 & 66 & 102 \\
\hline VI250(1)-70 & 138,69 & 0,41 & 1,2 & 66 & 103 \\
\hline VI250(1)-71 & 140,76 & 0,42 & 1,1 & 66 & 103 \\
\hline VI250(1)-72 & 142,84 & 0,43 & 1,1 & 65 & 101 \\
\hline VI250(1)-73 & 144,88 & 0,37 & 1,2 & 64 & 100 \\
\hline VI250(1)-74 & 146,88 & 0,33 & 1,2 & 65 & 102 \\
\hline VI250(1)-75 & 148,98 & 0,35 & 1,2 & 64 & 102 \\
\hline VI250(1)-76 & 150,86 & 0,36 & 1,3 & 64 & 98 \\
\hline VI250(1)-77 & 153,07 & 0,42 & 1,3 & 64 & 97 \\
\hline VI250(1)-78 & 155,04 & 0,33 & 1,3 & 63 & 96 \\
\hline VI250(1)-79 & 157,06 & 0,32 & 1,3 & 63 & 97 \\
\hline VI250(1)-80 & 159,06 & 0,34 & 1,3 & 64 & 98 \\
\hline VI250(1)-81 & 161,00 & 0,36 & 1,3 & 61 & 94 \\
\hline VI250(1)-82 & 163,09 & 0,37 & 1,3 & 61 & 94 \\
\hline VI250(1)-83 & 165,01 & 0,39 & 1,2 & 58 & 93 \\
\hline VI250(1)-85 & 168,98 & 0,35 & 1,2 & 55 & 90 \\
\hline & & & & $2,9 \bullet 10^{-8}$ & $4,6 \bullet 10$ \\
\hline
\end{tabular}


Tab. A-20: Durchfluß-Experimente mit Kaolinit Versuche VI250(2) und VI250(3).

\section{VI250(2) \\ Kaolinit}

$\varnothing=0,63-2 \mu \mathrm{m}$

Einwaage Mineral [g]: 0,7508

Einwaage Lösung [g]: 248,9

Lösung: HCl, pH = 2

\begin{tabular}{|c|r|r|r|r|r|}
\hline & & & \multicolumn{1}{|c|}{$\mu$ mol/l } \\
\hline Probe & Tage & g/h & pH & \multicolumn{1}{|c|}{ Al } & \multicolumn{1}{|c|}{ Si } \\
\hline \hline VI250(2)-1 & 1,81 & 0,47 & 1,8 & 1,7 & 12 \\
\hline VI250(2)-2 & 3,97 & 0,45 & 1,9 & 1,8 & 13 \\
\hline VI250(2)-3 & 5,69 & 0,51 & 1,9 & 2,0 & 14 \\
\hline VI250(2)-4 & 5,99 & 0,49 & 2,0 & 2,1 & 14 \\
\hline VI250(2)-5 & 6,73 & 0,51 & 2,0 & 2,1 & 14 \\
\hline VI250(2)-6 & 7,08 & 0,51 & 2,0 & 2,2 & 14 \\
\hline VI250(2)-7 & 7,72 & 0,51 & 2,0 & 2,3 & 14 \\
\hline VI250(2)-8 & 12,08 & 0,49 & 2,0 & 2,8 & 15 \\
\hline VI250(2)-9 & 12,78 & 0,49 & 2,0 & 3,2 & 15 \\
\hline VI250(2)-10 & 14,77 & 0,51 & 2,0 & 3,6 & 17 \\
\hline VI250(2)-11 & 19,74 & 0,51 & 2,1 & 6,3 & 18 \\
\hline VI250(2)-12 & 22,73 & 0,53 & 2,1 & 6,9 & 18 \\
\hline VI250(2)-13 & 26,78 & 0,50 & 2,1 & 6,7 & 18 \\
\hline VI250(2)-14 & 29,98 & 0,23 & 2,1 & 7,5 & 19 \\
\hline VI250(2)-15 & 34,88 & 0,15 & 2,1 & 8,1 & 20 \\
\hline VI250(2)-16 & 36,09 & 0,70 & 2,1 & 8,3 & 20 \\
\hline VI250(2)-17 & 41,27 & 0,58 & 2,1 & 7,2 & 15 \\
\hline VI250(2)-18 & 42,00 & 0,89 & 2,0 & 6,8 & 14 \\
\hline VI250(2)-19 & 54,09 & 0,93 & 2,0 & 7,8 & 13 \\
\hline VI250(2)-20 & 61,09 & 1,14 & 2,0 & 7,7 & 12 \\
\hline VI250(2)-21 & 67,94 & 1,14 & 2,0 & 7,0 & 9,8 \\
\hline VI250(2)-22 & 74,73 & 1,14 & 1,9 & 5,5 & 3,4 \\
\hline VI250(2)-23 & 75,99 & 0,90 & 1,8 & 6,4 & 9,1 \\
\hline VI250(2)-24 & 79,15 & 0,91 & 1,9 & 6,4 & 11 \\
\hline VI250(2)-25 & 82,08 & 0,94 & 2,0 & 6,3 & 10 \\
\hline VI250(2)-26 & 86,11 & 0,93 & 2,0 & 6,2 & 10 \\
\hline VI250(2)-27 & 89,95 & 0,94 & 2,1 & 6,1 & 10 \\
\hline VI250(2)-28 & 93,23 & 0,91 & 2,1 & 5,9 & 9,8 \\
\hline VI250(2)-29 & 96,03 & 0,95 & 2,1 & 6,0 & 9,7 \\
\hline VI250(2)-30 & 100,12 & 0,99 & 2,1 & 6,1 & 9,5 \\
\hline VI250(2)-31 & 103,12 & 1,00 & 2,1 & 6,2 & 9,5 \\
\hline VI250(2)-32 & 111,07 & 1,01 & 2,1 & 7,5 & 8,7 \\
\hline VI250(2)-33 & 128,10 & 1,18 & 2,4 & 3,3 & 8,6 \\
\hline VI250(2)-34 & 131,02 & 0,96 & 2,1 & 3,2 & 8,5 \\
\hline VI250(2)-35 & 147,03 & 1,06 & 2,4 & 4,6 & 11 \\
\hline Rate [mo1/(g•h)] & & & $7,3 \bullet 10^{-9}$ & $1,3 \bullet 10$ \\
\hline-8
\end{tabular}

\section{VI250(3)}

Kaolinit

$\varnothing=0,63-2 \mu \mathrm{m}$

Einwaage Mineral [g]: 0,7508

Einwaage Lösung [g]: 248,9

Lösung: $\mathbf{H C l}, \mathbf{p H}=\mathbf{3}$

\begin{tabular}{|c|r|r|r|r|r|}
\hline & & & \multicolumn{1}{|c|}{$\boldsymbol{\mu m o l} / 1$} \\
\hline Probe & Tage & g/h & pH & Al & \multicolumn{1}{c|}{ Si } \\
\hline \hline VI250(3)-1 & 5,97 & 1,01 & 2,6 & 3,6 & 8,8 \\
\hline VI250(3)-2 & 8,93 & 1,04 & 2,6 & 3,5 & 8,1 \\
\hline VI250(3)-3 & 19,01 & 1,01 & 2,6 & 3,4 & 8,4 \\
\hline VI250(3)-4 & 20,96 & 0,90 & 2,6 & 3,3 & 8,1 \\
\hline VI250(3)-5 & 22,95 & 0,98 & 2,7 & 3,1 & 7,9 \\
\hline VI250(3)-6 & 25,85 & 1,46 & 2,7 & 2,9 & 7,3 \\
\hline VI250(3)-7 & 27,95 & 0,94 & 2,7 & 2,0 & 12 \\
\hline VI250(3)-8 & 29,96 & 1,01 & 2,7 & 2,2 & 8,9 \\
\hline VI250(3)-9 & 33,06 & 1,04 & 2,8 & 1,9 & 7,1 \\
\hline VI250(3)-10 & 39,88 & 1,12 & 2,8 & 1,9 & 6,2 \\
\hline VI250(3)-11 & 41,91 & 1,01 & 2,8 & 1,8 & 5,7 \\
\hline VI250(3)-12 & 42,93 & 1,04 & 2,8 & 1,7 & 5,3 \\
\hline VI250(3)-13 & 48,94 & 1,03 & 2,9 & 2,0 & 5,5 \\
\hline VI250(3)-14 & 50,87 & 1,17 & 2,9 & 1,8 & 4,9 \\
\hline VI250(3)-15 & 55,11 & 1,07 & 2,9 & 1,9 & 4,7 \\
\hline VI250(3)-16 & 57,01 & 1,09 & 2,9 & 2,0 & 4,8 \\
\hline VI250(3)-17 & 60,92 & 1,08 & 2,9 & 2,2 & 4,6 \\
\hline VI250(3)-18 & 62,92 & 1,02 & 2,9 & 2,4 & 4,8 \\
\hline VI250(3)-19 & 68,01 & 1,09 & 2,9 & 2,4 & 4,3 \\
\hline VI250(3)-20 & 69,96 & 1,13 & 2,7 & 2,0 & 8,7 \\
\hline VI250(3)-21 & 71,99 & 1,04 & 2,8 & 1,8 & 7,8 \\
\hline VI250(3)-22 & 76,99 & 1,05 & 2,8 & 2,1 & 8,5 \\
\hline VI250(3)-23 & 78,88 & 1,02 & 2,8 & 2,2 & 8,5 \\
\hline VI250(3)-24 & 81,97 & 1,07 & 2,9 & 2,1 & 8,9 \\
\hline VI250(3)-25 & 84,04 & 1,13 & 2,9 & 2,0 & 8,5 \\
\hline VI250(3)-26 & 85,91 & 0,93 & 2,9 & 2,0 & 8,0 \\
\hline VI250(3)-27 & 89,89 & 0,89 & 2,8 & 1,8 & 10 \\
\hline VI250(3)-28 & 92,02 & 1,14 & 2,9 & 2,1 & 10 \\
\hline VI250(3)-29 & 96,00 & 1,20 & 2,9 & 2,0 & 8,0 \\
\hline VI250(3)-30 & 98,93 & 1,10 & 2,9 & 1,1 & 6,7 \\
\hline VI250(3)-31 & 102,98 & 1,21 & 2,9 & 2,0 & 8,7 \\
\hline VI250(3)-32 & 105,01 & 1,13 & 2,9 & 2,0 & 7,1 \\
\hline VI250(3)-33 & 106,92 & 1,14 & 2,9 & 2,2 & 7,0 \\
\hline VI250(3)-34 & 110,91 & 1,11 & 2,9 & 2,1 & 7,3 \\
\hline VI250(3)-35 & 114,02 & 1,19 & 2,9 & 2,1 & 6,6 \\
\hline VI250(3)-36 & 116,99 & 0,77 & 2,8 & 2,5 & 7,2 \\
\hline Rate [mol/g•h)] & & & & \\
\hline
\end{tabular}


Tab. A-21: Durchfluß-Experimente mit Kaolinit Versuche VI250(4) und X(1).

\section{VI250(4) \\ Kaolinit}

$\varnothing=0,63-2 \mu \mathrm{m}$

Einwaage Mineral [g]: 0,7508

Einwaage Lösung [g]: 248,9

Lösung: $\mathbf{H C l}, \mathbf{p H}=\mathbf{3 , 3}$

\begin{tabular}{|c|c|c|c|c|c|}
\hline & & & & \multicolumn{2}{|l|}{$\mu \mathrm{mol} / 1$} \\
\hline Probe & Tage & $\mathbf{g} / \mathbf{h}$ & $\mathrm{pH}$ & $\overline{\mathbf{A l}}$ & $\mathbf{S i}$ \\
\hline \begin{tabular}{|l|} 
VI250(4)-1 \\
\end{tabular} & $\overline{7,63}$ & $\overline{1,12}$ & $\overline{3,4}$ & 0,61 & $\overline{5,8}$ \\
\hline \begin{tabular}{|l|} 
VI250(4)-2 \\
\end{tabular} & 12,62 & 1,14 & 3,4 & 0,75 & $\overline{5,1}$ \\
\hline \begin{tabular}{|l|} 
VI250(4)-3 \\
\end{tabular} & 19,69 & 1,18 & 3,3 & 0,82 & 6,3 \\
\hline VI250(4)-4 & 26,65 & 1,18 & 3,4 & 1,3 & 7,1 \\
\hline VI250(4)-5 & 28,67 & 1,15 & 3,5 & 1,5 & 6,9 \\
\hline VI250(4)-6 & 33,69 & 1,16 & 3,5 & 1,9 & 7,1 \\
\hline VI250(4)-7 & 40,68 & 1,16 & 3,5 & 2,3 & 7,1 \\
\hline VI250(4)-8 & 47,67 & 1,17 & 3,4 & 2,7 & 6,9 \\
\hline \begin{tabular}{|l|} 
VI250(4)-9 \\
\end{tabular} & 112,79 & 1,11 & 3,1 & 2,3 & $\overline{5,1}$ \\
\hline VI250(4)-10 & 121,78 & 1,15 & 3,1 & 2,2 & 5,1 \\
\hline VI250(4)-11 & 125,78 & 1,13 & 3,1 & 2,3 & 5,4 \\
\hline VI250(4)-12 & 131,80 & 1,16 & 3,2 & 1,9 & 5,1 \\
\hline VI250(4)-13 & 138,67 & 1,26 & 3,2 & 1,8 & 4,8 \\
\hline VI250(4)-14 & 145,72 & 1,16 & 3,2 & 1,9 & 4,9 \\
\hline VI250(4)-15 & 153,64 & 1,05 & 3,2 & 2,2 & 5,4 \\
\hline VI250(4)-16 & 159,67 & 1,16 & 3,2 & 2,3 & 5,2 \\
\hline VI250(4)-17 & 166,61 & 1,19 & 3,2 & 2,5 & 5,3 \\
\hline ate $[\mathrm{mol} /(\mathrm{g}$ & & & & $\overline{3 \cdot 10^{-9}}$ & $7,9 \cdot 10^{-9}$ \\
\hline
\end{tabular}

\section{$\mathbf{X}(\mathbf{1})$}

\section{Kaolinit}

$\emptyset=0,63-2 \mu \mathrm{m}$

Einwaage Mineral [g]: 0,3479

Einwaage Lösung [g]: 89,4

Lösung: $\mathrm{C}_{6} \mathbf{H}_{8} \mathrm{O}_{7} \bullet \mathbf{H}_{2} \mathrm{O} ; \mathbf{M}=\mathbf{0 , 0 1}$

\begin{tabular}{|l|r|r|r|r|r|}
\hline & & & \multicolumn{1}{|c|}{$\mu \mathrm{mol} / 1$} \\
\hline \multicolumn{1}{|c|}{ Probe } & Tage & g/h & pH & Al & \multicolumn{1}{c|}{ Si } \\
\hline $\mathrm{X}(1)-1$ & 0,14 & 1,27 & 1,7 & 0,98 & 4,8 \\
\hline $\mathrm{X}(1)-2$ & 0,98 & 1,01 & 2,0 & 1,2 & 13 \\
\hline $\mathrm{X}(1)-3$ & 4,83 & 4,51 & 2,1 & 1,3 & 16 \\
\hline $\mathrm{X}(1)-4$ & 6,94 & 1,80 & 2,1 & 0,82 & 14 \\
\hline $\mathrm{X}(1)-5$ & 8,15 & 1,71 & 2,2 & 0,75 & 12 \\
\hline $\mathrm{X}(1)-6$ & 13,33 & 0,98 & 2,2 & 0,53 & 5,7 \\
\hline $\mathrm{X}(1)-7$ & 14,06 & 1,44 & 2,4 & 0,68 & 6,2 \\
\hline $\mathrm{X}(1)-8$ & 26,15 & 0,72 & 2,4 & 3,7 & 10 \\
\hline $\mathrm{X}(1)-9$ & 31,11 & 3,84 & 2,4 & 7,1 & 19 \\
\hline $\mathrm{X}(1)-10$ & 39,95 & 2,47 & 2,4 & 2,0 & 3,7 \\
\hline $\mathrm{X}(1)-11$ & 45,95 & 2,49 & 2,5 & 2,0 & 3,6 \\
\hline $\mathrm{X}(1)-12$ & 47,97 & 2,14 & 2,5 & 2,0 & 3,6 \\
\hline $\mathrm{X}(1)-13$ & 50,84 & 2,90 & 2,0 & 2,2 & 4,3 \\
\hline $\mathrm{X}(1)-14$ & 54,04 & 2,42 & 2,2 & 2,2 & 4,2 \\
\hline $\mathrm{X}(1)-15$ & 58,05 & 2,33 & 2,2 & 2,2 & 4,2 \\
\hline $\mathrm{X}(1)-16$ & 61,98 & 2,37 & 2,3 & 2,3 & 4,1 \\
\hline $\mathrm{X}(1)-17$ & 64,96 & 2,40 & 2,3 & 2,3 & 3,9 \\
\hline $\mathrm{X}(1)-18$ & 68,01 & 2,36 & 2,4 & 2,3 & 3,8 \\
\hline $\mathrm{X}(1)-19$ & 72,18 & 2,07 & 2,4 & 2,4 & 3,7 \\
\hline $\mathrm{X}(1)-20$ & 74,97 & 2,68 & 2,4 & 2,2 & 3,6 \\
\hline $\mathrm{X}(1)-21$ & 82,98 & 2,41 & 2,5 & 2,1 & 3,3 \\
\hline & & & & & \\
\hline Rate [mol/(g•h)] & & & $1,5 \bullet 10^{-8}$ & $2,6 \bullet 10^{-8}$ \\
\hline
\end{tabular}


Tab. A-22: Durchfluß-Experimente mit Kaolinit Versuch X(2).

$\mathbf{X}(2)$

Kaolinit

$\varnothing=0,63-2 \mu \mathrm{m}$

Einwaage Mineral [g]: 0,3479

Einwaage Lösung [g]: 89,4

Lösung: $\mathrm{C}_{6} \mathbf{H}_{8} \mathrm{O}_{7} \cdot \mathbf{H}_{2} \mathrm{O} ; \mathbf{M}=\mathbf{0 , 0 5}$

\begin{tabular}{|c|c|c|c|c|c|}
\hline & & & & \multicolumn{2}{|l|}{$\mu \mathrm{mol} / \mathrm{l}$} \\
\hline Probe & Tage & $\mathrm{g} / \mathrm{h}$ & pH & $\mathbf{A l}$ & $\mathbf{S i}$ \\
\hline $\mathrm{X}(2)-1$ & 17,06 & 2,30 & 2,2 & 6,3 & 13 \\
\hline $\mathrm{X}(2)-2$ & 20,04 & 2,49 & 2,2 & 3,8 & 6,3 \\
\hline$X(2)-3$ & 35,95 & 2,83 & $\overline{2,2}$ & 4,7 & 8,7 \\
\hline$\overline{X(2)-4}$ & 41,95 & 2,74 & $\overline{2,2}$ & 3,3 & 6,3 \\
\hline $\mathrm{X}(2)-5$ & 44,89 & 2,85 & 2,2 & 3,1 & 6,1 \\
\hline$X(2)-6$ & 54,95 & 2,62 & 2,3 & 3,5 & 8,5 \\
\hline $\mathrm{X}(2)-7$ & 57,08 & 1,94 & $\overline{2,3}$ & 3,7 & 8,9 \\
\hline$X(2)-8$ & 58,97 & 2,71 & 2,3 & 3,3 & 8,7 \\
\hline $\mathrm{X}(2)-9$ & 61,93 & 2,65 & 2,2 & 2,9 & 7,5 \\
\hline $\mathrm{X}(2)-10$ & 63,95 & 2,54 & $\overline{2,2}$ & 1,8 & 4,6 \\
\hline$X(2)-11$ & 86,92 & 2,91 & 2,2 & 1,9 & 4,9 \\
\hline 10 & & & & $20 \cdot 10^{-8}$ & \\
\hline
\end{tabular}




\section{Montmorillonit}

Tab. A-23: Batch-Experimente mit Montmorillonit Versuche 16 und 17 (*: keine Auflösungsrate berechnet, da die Konzentrationen durch eine Ionenaustauschreaktion bedingt sind).

16

Montmorillonit

$\emptyset=<0,63 \mu \mathrm{m}$

Einwaage Mineral [g]: 0,1509

Einwaage Lösung [g]: 100,1309

Lösung: $\mathrm{H}_{2} \mathrm{SO}_{4}, \mathbf{p H}=\mathbf{1}$

\begin{tabular}{|c|c|c|c|c|c|c|c|}
\hline \multirow[b]{2}{*}{ Probe } & \multirow[b]{2}{*}{ Tage } & \multirow[b]{2}{*}{ pH } & \multicolumn{5}{|l|}{$\mu \mathrm{mol} / 1$} \\
\hline & & & Al & $\mathbf{F e}$ & Mg & $\mathbf{N a}$ & $\mathbf{S} \mathbf{i}$ \\
\hline 16-1 & 19,27 & 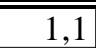 & 186 & 31 & 144 & 1.237 & 305 \\
\hline $16-2$ & 40,20 & 1,3 & 592 & 70 & 129 & 1.160 & 1.179 \\
\hline $16-3$ & 97,20 & 1,0 & 2.242 & 201 & 336 & 1.040 & 4.170 \\
\hline $16-4$ & 142,20 & 1,2 & 3.679 & 251 & 406 & 990 & 6.100 \\
\hline $16-5$ & 184,06 & 0,9 & 3.639 & 411 & 594 & 1.028 & 6.230 \\
\hline $16-6$ & 416,18 & 0,9 & 4.127 & 493 & 635 & 888 & 8.684 \\
\hline $16-7$ & 539,10 & 1,0 & 4.133 & 639 & 846 & 1.312 & 8.860 \\
\hline \multicolumn{3}{|c|}{ Rate $[\mathrm{mol} /(\mathrm{g} \bullet \mathrm{h})]<142$ Tage } & $7,9 \cdot 10^{-7}$ & $5,1 \cdot 10^{-8}$ & $6,7 \cdot 10^{-8}$ & $*$ & $1,3 \cdot 10^{-6}$ \\
\hline \multicolumn{3}{|c|}{ Rate $[\mathrm{mol} /(\mathrm{g} \cdot \mathrm{h})] 142-416$ Tage } & $5,0 \bullet 10^{-8}$ & $1,9 \cdot 10^{-8}$ & $1,7 \cdot 10^{-8}$ & * & $2,7 \cdot 10^{-7}$ \\
\hline \multicolumn{3}{|c|}{ Rate $[\mathrm{mol} /(\mathrm{g} \bullet \mathrm{h})]$ 416-539 Tage } & $1,3 \cdot 10^{-9}$ & $3,3 \cdot 10^{-8}$ & $4,7 \cdot 10^{-8}$ & $*$ & $4,0 \cdot 10^{-8}$ \\
\hline
\end{tabular}

17

Montmorillonit

$\varnothing=<0,63 \mu \mathrm{m}$

Einwaage Mineral [g]: 0,1507

Einwaage Lösung [g]: 100,0277

Lösung: $\mathbf{H}_{2} \mathbf{S O}_{4}, \mathbf{p H}=\mathbf{2}$

\begin{tabular}{|c|c|c|c|c|c|c|c|}
\hline \multirow[b]{2}{*}{ Probe } & \multirow[b]{2}{*}{ Tage } & \multirow[b]{2}{*}{ pH } & \multicolumn{5}{|l|}{$\mu \mathrm{mol} / 1$} \\
\hline & & & Al & $\mathbf{F e}$ & Mg & $\mathbf{N a}$ & $\mathbf{S}$ i \\
\hline $17-1$ & 19,17 & $\overline{1,9}$ & 2,4 & 6,0 & 53 & 1.250 & 188 \\
\hline $17-2$ & 40,11 & 1,8 & 2,4 & 6,6 & 60 & 1.061 & 212 \\
\hline $17-3$ & 142,10 & 2,2 & 12 & 17 & 95 & 1.070 & 435 \\
\hline $17-4$ & 183,97 & 1,9 & 18 & 19 & 86 & 1.126 & 466 \\
\hline $17-5$ & 341,00 & 1,7 & 110 & 37 & 162 & 1.232 & 693 \\
\hline $17-6$ & 416,09 & 2,0 & 158 & 41 & 163 & 929 & 1.025 \\
\hline $17-7$ & 539,01 & 2,0 & 224 & 60 & 230 & 1.243 & 1.218 \\
\hline Rate [mol & & & $1,2 \cdot 10^{-8}$ & $2,8 \cdot 10^{-9}$ & $9,0 \cdot 10^{-9}$ & * & $5,5 \cdot 10^{-8}$ \\
\hline
\end{tabular}


Tab. A-24: Batch-Experimente mit Montmorillonit Versuche 26 und 30 (*: keine Auflösungsrate berechnet, da die Konzentrationen durch eine Ionenaustauschreaktion bedingt sind).

26

\section{Montmorillonit}

$\varnothing=<0,63 \mu \mathrm{m}$

Einwaage Mineral [g]: 0,1501

Einwaage Lösung [g]: 100,0008

Lösung: $\mathrm{C}_{6} \mathbf{H}_{8} \mathbf{O}_{7} \cdot \mathbf{H}_{2} \mathbf{O}, \mathbf{M}=\mathbf{0 , 0 1}$

\begin{tabular}{|c|c|c|c|c|c|c|c|}
\hline \multirow[b]{2}{*}{ Probe } & \multirow[b]{2}{*}{ Tage } & \multirow[b]{2}{*}{$\mathbf{p H}$} & \multicolumn{5}{|l|}{$\mu \mathrm{mol} / 1$} \\
\hline & & & Al & $\mathbf{F e}$ & Mg & $\mathbf{N a}$ & $\mathbf{S} \mathbf{i}$ \\
\hline $26-1$ & 8,08 & 2,6 & 16 & 8,2 & 9,1 & 1.023 & 161 \\
\hline $26-2$ & 29,02 & 2,6 & 20 & 13 & 14 & 976 & 201 \\
\hline $26-3$ & 89,99 & 2,6 & 63 & 22 & 10 & 1.003 & 264 \\
\hline $26-4$ & 131,02 & 2,8 & 114 & 28 & n.b. & 940 & 350 \\
\hline $26-5$ & 187,83 & 2,8 & 137 & 31 & n.b. & 944 & 415 \\
\hline $26-6$ & 330,01 & 3,2 & 286 & 60 & 15 & 890 & 575 \\
\hline $26-7$ & 404,99 & 3,2 & 316 & 57 & 22 & 775 & 844 \\
\hline \multicolumn{2}{|c|}{ Rate $[\mathrm{mol} /(\mathrm{g} \bullet \mathrm{h})]$} & & $2,2 \cdot 10^{-8}$ & $3,7 \cdot 10^{-9}$ & $6,6 \cdot 10^{-10}$ & $*$ & $4,4 \cdot 10^{-8}$ \\
\hline
\end{tabular}

30

Montmorillonit

$\varnothing=<0,63 \mu \mathrm{m}$

Einwaage Mineral [g]: 0,1503

Einwaage Lösung [g]: 100,0036

Lösung: $\mathbf{H}_{2} \mathbf{S O}_{4}, \mathbf{p H}=\mathbf{3}$

\begin{tabular}{|c|c|c|c|c|c|c|c|}
\hline & & & $\mu \mathrm{mol} / 1$ & & & & \\
\hline Probe & Tage & $\mathbf{p H}$ & $\overline{A l}$ & $\mathbf{F e}$ & Mg & $\mathbf{N a}$ & $\mathbf{S i}$ \\
\hline $30-1$ & 2,22 & 4,0 & 2,9 & 3,3 & 5,5 & 450 & 94 \\
\hline $30-2$ & 17,13 & 4,0 & 3,9 & 0,45 & 1,5 & 464 & 110 \\
\hline $30-3$ & 159,32 & 3,5 & $\overline{4,5}$ & 0,66 & 1,7 & 481 & 183 \\
\hline $30-4$ & 286,02 & 4,9 & 6,0 & 7,0 & n.b. & 417 & 238 \\
\hline $30-5$ & 357,22 & 4,7 & 3,9 & 0,96 & 4,4 & 505 & 272 \\
\hline \multicolumn{3}{|c|}{ Rate $[\mathrm{mol} /(\mathrm{g} \bullet \mathrm{h})]$ 17-357 Tage } & $5,9 \cdot 10^{-11}$ & $2,5 \cdot 10^{-10}$ & $2,5 \cdot 10^{-10}$ & $*$ & $1,3 \cdot 10^{-8}$ \\
\hline
\end{tabular}


Tab. A-25: Batch-Experimente mit Montmorillonit Versuche 37 und 38 (*: keine Auflösungsrate berechnet, da die Konzentrationen durch eine Ionenaustauschreaktion bedingt sind).

37

\section{Montmorillonit}

$\varnothing=<0,63 \mu \mathrm{m}$

Einwaage Mineral [g]: 0,1499

Einwaage Lösung [g]: 100,0110

Lösung: $\mathrm{HNO}_{3}, \mathbf{p H}=\mathbf{1}$

\begin{tabular}{|c|c|c|c|c|c|c|c|}
\hline \multirow[b]{2}{*}{ Probe } & \multirow[b]{2}{*}{ Tage } & \multirow[b]{2}{*}{ pH } & \multicolumn{5}{|l|}{$\mu \mathrm{mol} / 1$} \\
\hline & & & A I & $\mathbf{F e}$ & Mg & $\mathbf{N a}$ & $\mathbf{S} \mathbf{i}$ \\
\hline $37-1$ & 2,27 & $\overline{1,0}$ & 51 & 10 & 84 & 1.053 & 93 \\
\hline $37-2$ & 17,16 & 1,0 & 92 & 17 & 91 & 1.054 & 237 \\
\hline $37-3$ & 198,19 & 1,2 & 2.357 & 227 & 341 & 964 & 5.018 \\
\hline $37-4$ & 235,13 & 1,1 & 2.903 & 357 & 514 & 932 & 6.685 \\
\hline $37-5$ & 357,26 & 1,1 & 3.017 & 489 & 721 & 1.440 & 6.975 \\
\hline \multicolumn{3}{|c|}{ Rate $[\mathrm{mol} /(\mathrm{g} \bullet \mathrm{h})]$ 2-17 Tage } & $7,7 \cdot 10^{-8}$ & $1,3 \cdot 10^{-8}$ & $1,3 \cdot 10^{-8}$ & $*$ & $2,7 \cdot 10^{-7}$ \\
\hline \multicolumn{3}{|c|}{ Rate $[\mathrm{mol} /(\mathrm{g} \bullet \mathrm{h})] 17-198$ Tage } & $3,5 \cdot 10^{-7}$ & $3,2 \cdot 10^{-8}$ & $3,8 \cdot 10^{-8}$ & $*$ & $7,3 \cdot 10^{-7}$ \\
\hline \multicolumn{3}{|c|}{ Rate $[\mathrm{mol} /(\mathrm{g} \bullet \mathrm{h})] 198-235$ Tage } & $4,1 \cdot 10^{-7}$ & $9,8 \cdot 10^{-8}$ & $1,3 \cdot 10^{-7}$ & $*$ & $1,3 \cdot 10^{-6}$ \\
\hline \multicolumn{3}{|c|}{ Rate $[\mathrm{mol} /(\mathrm{g} \bullet \mathrm{h})]$ 235-357 Tage } & $2,6 \cdot 10^{-8}$ & $3,0 \cdot 10^{-8}$ & $4,7 \cdot 10^{-8}$ & $*$ & $6,6 \cdot 10^{-8}$ \\
\hline
\end{tabular}

38

Montmorillonit

$\varnothing=<0,63 \mu \mathrm{m}$

Einwaage Mineral [g]: 0,1491

Einwaage Lösung [g]: 100,0028

Lösung: $\mathbf{H N O}_{3}, \mathbf{p H}=\mathbf{2}$

\begin{tabular}{|c|c|c|c|c|c|c|c|}
\hline & & & \multicolumn{5}{|l|}{$\mu \mathrm{mol} / 1$} \\
\hline Probe & Tage & $\mathrm{pH}$ & Al & $\mathbf{F e}$ & Mg & $\mathrm{Na}$ & $\mathbf{S i}$ \\
\hline $38-1$ & 2,27 & 2,0 & $<0,37$ & 2,7 & 17 & 1.049 & 68 \\
\hline $38-2$ & 17,16 & 2,1 & 0,71 & 4,6 & 24 & 1.061 & 175 \\
\hline $38-3$ & 160,13 & 2,1 & 3,9 & 15 & 95 & 1.158 & 372 \\
\hline $38-4$ & 235,13 & 2,1 & 16 & 20 & 119 & 944 & 634 \\
\hline $38-5$ & 357,26 & 2,0 & 52 & 33 & 192 & 1.260 & 799 \\
\hline & & & 4.10 & 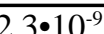 & $15 \cdot 10^{\circ}$ & & $\bullet \prod(1$ \\
\hline
\end{tabular}


Tab. A-26: Batch-Experimente mit Montmorillonit Versuche 39 und 45 (*: keine Auflösungsrate berechnet, da die Konzentrationen durch eine Ionenaustauschreaktion bedingt sind). IN kennzeichnet die Input-Lösung.

39

\section{Montmorillonit}

$\varnothing=<0,63 \mu \mathrm{m}$

Einwaage Mineral [g]: 0,1492

Einwaage Lösung [g]: 100,0026

Lösung: $\mathrm{HNO}_{3}, \mathbf{p H}=3$

\begin{tabular}{|c|c|c|c|c|c|c|c|}
\hline \multirow[b]{2}{*}{ Probe } & \multirow[b]{2}{*}{ Tage } & \multirow[b]{2}{*}{$\mathbf{p H}$} & \multicolumn{5}{|l|}{$\mu \mathrm{mol} / 1$} \\
\hline & & & Al & $\mathbf{F e}$ & Mg & $\mathbf{N a}$ & $\mathbf{S} \mathbf{i}$ \\
\hline $39-1$ & 2,27 & 3,6 & $\overline{2,6}$ & 0,29 & $<0,82$ & 430 & 87 \\
\hline $39-2$ & 17,16 & 4,3 & 5,1 & 0,61 & $<0,82$ & 409 & 107 \\
\hline $39-3$ & 160,13 & 3,1 & 4,4 & 0,65 & 1,5 & 434 & 163 \\
\hline $39-4$ & 235,13 & 5,0 & 4,9 & 0,81 & 3,8 & 504 & 213 \\
\hline $39-5$ & 357,26 & 4,5 & 2,9 & 0,75 & 4,1 & n.b. & 218 \\
\hline \multicolumn{3}{|c|}{ Rate $[\mathrm{mol} /(\mathrm{g} \bullet \mathrm{h})] 17-235$ Tage } & $-4,0 \cdot 10^{-10}$ & $2,3 \cdot 10^{-11}$ & $8,6 \cdot 10^{-10}$ & $*$ & $1,3 \cdot 10^{-8}$ \\
\hline \multicolumn{3}{|c|}{ Rate $[\mathrm{mol} /(\mathrm{g} \bullet \mathrm{h})] 235-357$ Tage } & $-4,6 \cdot 10^{-10}$ & $-1,4 \cdot 10^{-11}$ & $6,9 \cdot 10^{-11}$ & $*$ & $1,1 \cdot 10^{-9}$ \\
\hline
\end{tabular}

\section{5}

\section{Montmorillonit}

$\varnothing=<0,63 \mu \mathrm{m}$

Einwaage Mineral [g]: 0,0690

Einwaage Lösung [g]: 40,0085

Lösung: abgepreßte Porenlösung Solling

0-15 cm Tiefe

$\mathrm{pH}=3,5$

DOC $=39,15 \mathrm{mg} / \mathrm{l}$

\begin{tabular}{|c|r|r|r|r|r|r|r|}
\hline & \multicolumn{9}{|c|}{$\mu \mathrm{mol} / 1$} \\
\hline Probe & \multicolumn{1}{c|}{ Tage } & \multicolumn{1}{c|}{$\mathbf{p H}$} & \multicolumn{1}{c|}{ A l } & \multicolumn{1}{c|}{ Fe } & \multicolumn{1}{c|}{ M g } & \multicolumn{1}{c|}{ Na } & \multicolumn{1}{c|}{$\mathbf{S ~ i ~}$} \\
\hline \hline IN & 0 & 3,5 & 20 & 6,3 & 59 & 328 & 362 \\
\hline $45-1$ & 24,94 & 5,3 & 3,5 & 0,37 & 1,4 & 747 & 409 \\
\hline $45-2$ & 62,01 & 5,6 & 3,9 & 0,83 & 3,4 & 754 & 451 \\
\hline $45-3$ & 184,02 & 5,9 & 9,1 & 2,0 & 5,2 & 931 & 465 \\
\hline
\end{tabular}


Tab. A-27: Batch-Experimente mit Montmorillonit Versuch 49 (*: keine Auflösungsrate berechnet, da die Konzentrationen durch eine Ionenaustauschreaktion bedingt sind).

49

\section{Montmorillonit}

$\varnothing=<0,63 \mu \mathrm{m}$

Einwaage Mineral [g]: 0,1019

Einwaage Lösung [g]: 50,0032

Lösung: $\mathbf{H}_{2} \mathbf{O}^{* * * * *}, \mathbf{p H}>\mathbf{4}$

\begin{tabular}{|c|c|c|c|c|c|c|c|}
\hline & & & $\mu \mathrm{mol} / \mathrm{l}$ & & & & \\
\hline Probe & Tage & pH & Al & $\mathbf{F e}$ & Mg & $\mathbf{N a}$ & $\mathbf{S}$ i \\
\hline $49-1$ & 25,00 & 5,7 & $\overline{8,9}$ & 0,77 & $\overline{2,1}$ & 155 & $\overline{94}$ \\
\hline $49-2$ & 62,01 & 5,6 & 7,0 & 9,0 & 13 & n.b. & 290 \\
\hline $49-3$ & 115,01 & 5,8 & 41 & 8,3 & n.b. & 202 & 300 \\
\hline $49-4$ & 184,03 & 5,8 & 168 & 28 & 38 & 288 & 588 \\
\hline \multicolumn{2}{|c|}{ Rate $[\mathrm{mol} /(\mathrm{g} \bullet \mathrm{h})]$} & & $2,1 \cdot 10^{-8}$ & $3,2 \cdot 10^{-9}$ & $4,5 \cdot 10^{-9}$ & $*$ & $5,8 \cdot 10^{-8}$ \\
\hline
\end{tabular}


Tab. A-28: Durchfluß-Experimente mit Montmorillonit Versuch VIII(1) (*: keine Auflösungsrate berechnet, da die Konzentrationen durch eine Ionenaustauschreaktion bedingt sind).

\section{VIII(1)}

\section{Montmorillonit}

$\varnothing=<0,63 \mu \mathrm{m}$

Einwaage Mineral [g]: 0,4997

Einwaage Lösung [g]: 239,0

Lösung: $\mathrm{H}_{2} \mathrm{SO}_{4}, \mathbf{p H}=1$

\begin{tabular}{|c|c|c|c|c|c|c|c|c|}
\hline & & & & $\mu \mathrm{mol} / \mathrm{l}$ & & & & \\
\hline Probe & Tage & $\mathrm{g} / \mathrm{h}$ & pH & Al & $\mathbf{F e}$ & Mg & $\mathbf{N a}$ & $\underline{\mathrm{Si}}$ \\
\hline VIII(1)-1 & 0,70 & 0,89 & 1,3 & 56 & 17 & 141 & 1.438 & $\overline{79}$ \\
\hline VIII(1)-2 & 1,89 & 1,09 & 1,3 & 90 & 23 & 138 & 1.312 & 153 \\
\hline VIII (1)-3 & 5,74 & 6,08 & 1,3 & 107 & 25 & 115 & 986 & 186 \\
\hline VIII(1)-4 & 7,85 & 1,50 & 1,3 & 111 & 25 & 95 & $\overline{744}$ & 200 \\
\hline VIII(1)-5 & 9,07 & 1,71 & 1,3 & 113 & 25 & 87 & 639 & 207 \\
\hline VIII $(1)-6$ & 14,24 & 1,07 & 1,6 & 111 & 18 & 47 & 254 & 218 \\
\hline VIII(1)-7 & 14,97 & 1,83 & 1,2 & 101 & 17 & 43 & 224 & 202 \\
\hline VIII(1)-8 & 40,86 & 2,38 & 1,2 & 94 & 12 & 16 & $<4,4$ & 206 \\
\hline VIII $(1)-9$ & 46,87 & 2,50 & 1,2 & 85 & 11 & 14 & $<4,4$ & 190 \\
\hline VIII(1)-10 & 48,89 & 2,12 & 1,2 & 86 & 11 & 14 & $<4,4$ & 192 \\
\hline VIII(1)-11 & 51,76 & 2,82 & 1,1 & 91 & 11 & 14 & $<4,4$ & 217 \\
\hline VIII(1)-12 & 54,95 & 2,36 & 1,1 & 92 & 11 & 14 & $<4,4$ & 220 \\
\hline VIII(1)-13 & 58,97 & 2,28 & 1,1 & 87 & 11 & 13 & $<4,4$ & 213 \\
\hline VIII(1)-14 & 62,89 & 2,28 & 1,1 & 91 & 12 & 14 & $<4,4$ & 221 \\
\hline VIII(1)-15 & 65,87 & 2,32 & 1,1 & 90 & 12 & 14 & $<4,4$ & 219 \\
\hline VIII(1)-16 & 68,92 & 2,29 & 1,1 & 89 & 11 & 14 & $<4,4$ & 218 \\
\hline VIII(1)-17 & 73,09 & 2,14 & 1,1 & 87 & 12 & 13 & $<4,4$ & 215 \\
\hline VIII(1)-18 & 75,89 & 2,60 & 1,1 & 86 & 11 & 13 & $<4,4$ & 213 \\
\hline \multicolumn{2}{|c|}{ Rate $[\mathrm{mol} /(\mathrm{g} \bullet \mathrm{h})]$} & & & $4,1 \cdot 10^{-7}$ & $5,3 \cdot 10^{-8}$ & $6,3 \cdot 10^{-8}$ & * & $1,0 \cdot 10^{-6}$ \\
\hline
\end{tabular}


Tab. A-29: Durchfluß-Experimente mit Montmorillonit Versuch VIII(2) (*: keine Auflösungsrate berechnet, da die Konzentrationen durch eine Ionenaustauschreaktion bedingt sind).

VIII(2)

Montmorillonit

$\emptyset=<0,63 \mu \mathrm{m}$

Einwaage Mineral [g]: 0,4997

Einwaage Lösung [g]: 239,0

Lösung: $\mathbf{H}_{2} \mathrm{SO}_{4}, \mathbf{p H}=\mathbf{2}$

\begin{tabular}{|c|c|c|c|c|c|c|c|c|}
\hline & & & & $\mu \mathrm{mol} / 1$ & & & & \\
\hline Probe & Tage & g/h & pH & Al & $\mathbf{F e}$ & Mg & $\mathrm{Na}$ & $\mathbf{S i}$ \\
\hline VIII(2)-1 & 17,06 & 2,27 & 1,9 & 3,2 & 2,5 & $<0,82$ & $\overline{<<4,4}$ & 95 \\
\hline VIII(2)-2 & 20,04 & 2,41 & 2,0 & 1,7 & 2,0 & $<0,82$ & $<4,4$ & 76 \\
\hline VIII(2)-3 & 35,95 & 2,71 & 2,0 & 19 & 7,4 & $<0,82$ & $<4,4$ & 122 \\
\hline VIII(2)-4 & 41,95 & 2,56 & 2,0 & 20 & 4,8 & $<0,82$ & $<4,4$ & 81 \\
\hline VIII(2)-5 & 44,89 & 2,72 & 1,9 & 38 & 5,9 & $<0,82$ & $<4,4$ & 75 \\
\hline VIII(2)-6 & 54,95 & 2,52 & 1,9 & 25 & 4,1 & $<0,82$ & $<4,4$ & 91 \\
\hline VIII(2)-7 & 57,08 & 1,93 & 1,9 & 27 & 4,6 & $<0,82$ & $<4,4$ & 90 \\
\hline VIII(2)-8 & 58,97 & 2,51 & 1,9 & 29 & 4,2 & $<0,82$ & $<4,4$ & 86 \\
\hline VIII(2)-9 & 61,93 & 2,54 & 1,9 & 29 & 3,9 & $<0,82$ & $<4,4$ & 77 \\
\hline VIII(2)-10 & 63,95 & 2,43 & 2,0 & 28 & 3,9 & 4,3 & $<4,4$ & 68 \\
\hline VIII(2)-11 & 66,08 & 1,98 & 2,0 & 29 & 4,4 & 4,2 & $<4,4$ & 67 \\
\hline VIII(2)-12 & 68,99 & 2,58 & 2,0 & 29 & 4,0 & 4,1 & $<4,4$ & 65 \\
\hline VIII(2)-13 & 75,99 & 2,48 & 2,0 & 27 & 3,7 & 3,8 & $<4,4$ & 65 \\
\hline VIII(2)-14 & 78,03 & 2,33 & 2,0 & 27 & 3,7 & 3,9 & $<4,4$ & 66 \\
\hline VIII(2)-15 & 78,97 & 2,56 & 2,0 & 26 & 3,6 & 3,8 & $<4,4$ & 65 \\
\hline VIII(2)-16 & 84,92 & 2,62 & 2,0 & 29 & 3,8 & 4,1 & $<4,4$ & 67 \\
\hline VIII(2)-17 & 86,92 & 2,82 & 2,0 & 29 & 3,8 & 4,1 & $<4,4$ & 69 \\
\hline VIII(2)-18 & 90,88 & 2,62 & 2,0 & 26 & 3,4 & 4,1 & $<4,4$ & 70 \\
\hline VIII(2)-19 & 92,86 & 2,63 & 2,0 & 26 & 3,4 & 4,2 & $<4,4$ & 72 \\
\hline VIII(2)-20 & 96,95 & 2,64 & 2,0 & 30 & 3,7 & 4,0 & $<4,4$ & 67 \\
\hline VIII(2)-21 & 99,01 & 2,57 & 1,9 & 32 & 4,0 & 4,0 & $<4,4$ & 65 \\
\hline VIII(2)-22 & 103,91 & 2,72 & 1,9 & 31 & 3,9 & 3,9 & $<4,4$ & 65 \\
\hline VIII(2)-23 & 105,97 & 2,57 & 1,9 & 33 & 3,6 & 4,0 & $<4,4$ & 76 \\
\hline VIII(2)-24 & 107,89 & 2,15 & 1,9 & 41 & 4,3 & 5,0 & $<4,4$ & 95 \\
\hline VIII(2)-25 & 112,94 & 2,54 & 1,9 & 31 & 3,2 & 3,7 & $<4,4$ & 73 \\
\hline VIII(2)-26 & 114,99 & 2,51 & 2,0 & 30 & 3,1 & 3,7 & $<4,4$ & 71 \\
\hline VIII(2)-27 & 117,96 & 2,71 & 2,0 & 30 & 3,1 & 3,5 & $<4,4$ & 70 \\
\hline VIII(2)-28 & 119,89 & 2,76 & 2,0 & 31 & 3,0 & 3,7 & $<4,4$ & 71 \\
\hline VIII(2)-29 & 122,03 & 2,13 & 1,9 & 35 & 3,5 & 4,1 & $<4,4$ & 78 \\
\hline VIII(2)-30 & 125,90 & 2,02 & 1,8 & 49 & 5,1 & 6,2 & $<4,4$ & 114 \\
\hline VIII(2)-31 & 128,01 & 2,80 & 1,9 & 34 & 3,4 & 4,2 & $<4,4$ & 79 \\
\hline VIII(2)-32 & 131,97 & 2,81 & 1,9 & 36 & 3,7 & 4,2 & $<4,4$ & 78 \\
\hline VIII(2)-33 & 135,05 & 2,68 & 1,9 & 36 & 3,7 & 4,2 & $<4,4$ & 77 \\
\hline VIII(2)-34 & 138,92 & 3,02 & 2,0 & 30 & 3,1 & 3,7 & $<4,4$ & 72 \\
\hline VIII(2)-35 & 141,13 & 2,59 & 2,0 & 29 & 3,2 & 4,0 & $<4,4$ & 75 \\
\hline VIII(2)-36 & 143,00 & 2,69 & 2,0 & 24 & 2,3 & 3,0 & $<4,4$ & 58 \\
\hline VIII(2)-37 & 146,92 & 2,72 & 1,9 & 28 & 3,0 & 3,2 & $<4,4$ & 58 \\
\hline VIII(2)-38 & 150,00 & 2,80 & 1,9 & 27 & 2,8 & 3,0 & $<4,4$ & 58 \\
\hline VIII(2)-39 & 152,89 & 2,79 & 2,0 & 23 & 2,4 & 3,0 & $<4,4$ & 57 \\
\hline \multicolumn{2}{|c|}{ Rate $[\mathrm{mol} /(\mathrm{g} \bullet \mathrm{h})]$} & & & $1,5 \cdot 10^{-7}$ & $1,6 \cdot 10^{-8}$ & $1,8 \cdot 10^{-8}$ & $*$ & $3,5 \cdot 10^{-7}$ \\
\hline
\end{tabular}


Tab. A-30: Durchfluß-Experimente mit Montmorillonit Versuch VIII(3) (*: keine Auflösungsrate berechnet, da die Konzentrationen durch eine Ionenaustauschreaktion bedingt sind).

\section{VIII(3)}

\section{Montmorillonit}

$\varnothing=<0,63 \mu \mathrm{m}$

Einwaage Mineral [g]: 0,4997

Einwaage Lösung [g]: 239,0

Lösung: $\mathbf{H}_{2} \mathrm{SO}_{4}, \mathbf{p H}=\mathbf{3}$

\begin{tabular}{|c|c|c|c|c|c|c|c|c|}
\hline & & & & $\mu \mathrm{mol} / \mathrm{l}$ & & & & \\
\hline Probe & Tage & $\mathrm{g} / \mathrm{h}$ & $\mathrm{pH}$ & Al & $\mathbf{F e}$ & Mg & $\mathrm{Na}$ & Si \\
\hline VIII(3)-1 & 7,50 & 2,88 & 3,1 & 2,1 & $<0,18$ & 3,1 & $<4,4$ & 29 \\
\hline VIII(3)-2 & 12,53 & 2,78 & 3,0 & 5,4 & 0,37 & 3,2 & $<4,4$ & 30 \\
\hline VIII(3)-3 & 19,58 & 2,92 & 3,0 & 9,1 & 0,91 & 2,9 & $<4,4$ & 32 \\
\hline VIII(3)-4 & 26,65 & 2,84 & 3,0 & 11 & $<0,18$ & 5,1 & $<4,4$ & 30 \\
\hline VIII(3)-5 & 33,55 & 2,85 & 3,0 & 11 & $<0,18$ & 4,8 & $<4,4$ & 27 \\
\hline VIII(3)-6 & 40,57 & 2,83 & 2,9 & 10 & $<0,18$ & 4,6 & $<4,4$ & 24 \\
\hline VIII(3)-7 & 47,54 & 2,87 & 3,0 & 10 & $<0,18$ & 4,5 & $<4,4$ & 23 \\
\hline VIII(3)-8 & 78,58 & 2,82 & 3,0 & 13 & 3,5 & 2,5 & $<4,4$ & 22 \\
\hline VIII(3)-9 & 83,62 & 2,78 & 3,0 & 12 & 3,3 & 2,5 & $<4,4$ & 20 \\
\hline VIII(3)-10 & 91,54 & 2,87 & 2,9 & 14 & 3,4 & 2,5 & $<4,4$ & 18 \\
\hline VIII(3)-11 & 97,60 & 2,79 & 2,9 & 15 & 3,4 & 2,4 & $<4,4$ & 19 \\
\hline VIII(3)-12 & 103,64 & 2,82 & 2,9 & 14 & 3,3 & 2,4 & $<4,4$ & 20 \\
\hline VIII(3)-13 & 112,60 & 2,91 & 3,0 & 7,4 & 1,0 & 2,4 & $<4,4$ & 22 \\
\hline VIII(3)-14 & 121,64 & 2,91 & 3,0 & 8,3 & 1,2 & 2,4 & $<4,4$ & 21 \\
\hline VIII(3)-15 & 125,60 & 2,80 & 2,9 & 8,2 & 1,2 & 2,7 & $<4,4$ & 21 \\
\hline VIII(3)-16 & 131,67 & 3,28 & 2,9 & 9,3 & 1,7 & 2,7 & $<4,4$ & 26 \\
\hline VIII(3)-17 & 138,67 & 2,33 & 2,9 & 9,4 & 1,2 & 2,6 & $<4,4$ & 23 \\
\hline VIII(3)-18 & 145,58 & 2,93 & 2,8 & 10 & 1,3 & 2,7 & $<4,4$ & 25 \\
\hline VIII(3)-19 & 153,64 & 2,19 & 2,8 & 10 & 1,4 & 2,2 & $<4,4$ & 24 \\
\hline VIII(3)-20 & 159,55 & 2,82 & 2,8 & 8,7 & 1,3 & 2,5 & $<4,4$ & 23 \\
\hline VIII(3)-21 & 166,61 & 2,59 & 2,9 & 9,8 & 1,4 & 2,8 & $<4,4$ & 24 \\
\hline \multicolumn{2}{|c|}{ Rate $[\mathrm{mol} /(\mathrm{g} \bullet \mathrm{h})]$} & & & $5,1 \cdot 10^{-8}$ & $7,4 \cdot 10^{-9}$ & $1,4 \cdot 10^{-8}$ & $*$ & $1,3 \cdot 10^{-7}$ \\
\hline
\end{tabular}


Tab. A-31: Durchfluß-Experimente mit Montmorillonit Versuch XIV (*: keine Auflösungsrate berechnet, da die Konzentrationen durch eine Ionenaustauschreaktion bedingt sind).

\section{XIV}

\section{Montmorillonit}

$\varnothing=<0,63 \mu \mathrm{m}$

Einwaage Mineral [g]: 0,3516

Einwaage Lösung [g]: 82,9

Lösung: $\mathrm{C}_{6} \mathrm{H}_{8} \mathrm{O}_{7} \cdot \mathbf{H}_{2} \mathrm{O}, \mathbf{M}=\mathbf{0 , 0 1}$

\begin{tabular}{|c|c|c|c|c|c|c|c|c|}
\hline & & & & \multicolumn{5}{|l|}{$\mu \mathrm{mol} / 1$} \\
\hline $\begin{array}{l}\text { Probe } \\
\end{array}$ & Tage & $\mathrm{g} / \mathbf{h}$ & pH & $\overline{\mathrm{Al}}$ & $\mathbf{F e}$ & $\mathbf{M g}$ & $\mathrm{Na}$ & $\overline{\mathrm{Si}}$ \\
\hline XIV-1 & 12,92 & 3,00 & 2,6 & 0,98 & 2,8 & 4 & $<4,4$ & 21 \\
\hline XIV-2 & 17,95 & 2,84 & 2,5 & 0,95 & 2,3 & 4,8 & $<4,4$ & 18 \\
\hline XIV-3 & 25,00 & 2,93 & 2,6 & 1,7 & 2,2 & |5,3 & 10 & 17 \\
\hline XIV-4 & 83,99 & 2,89 & 2,6 & 7,0 & 3,4 & n.b. & 6,6 & 17 \\
\hline XIV-5 & 96,96 & 2,84 & 2,5 & 8,7 & 3,5 & n.b. & $<4,4$ & 17 \\
\hline XIV-6 & 103,02 & 2,83 & 2,6 & 8,8 & 3,3 & n.b. & $<4,4$ & 16 \\
\hline XIV-7 & 109,06 & 2,91 & 2,5 & 9,4 & 3,3 & n.b. & $<4,4$ & 16 \\
\hline XIV-8 & 127,06 & 2,86 & 2,6 & 5,5 & 1,1 & 3,1 & $<4,4$ & 15 \\
\hline XIV-9 & 131,02 & 2,78 & 2,6 & 5,0 & $\overline{0,93}$ & 3,2 & $<4,4$ & 12 \\
\hline XIV-10 & 144,09 & 2,41 & 2,6 & 4,4 & 0,77 & 2,4 & $<4,4$ & 9,6 \\
\hline XIV-11 & 150,99 & 2,91 & 2,6 & 3,5 & 0,58 & 1,8 & $<4,4$ & 7,0 \\
\hline XIV-12 & 159,06 & 1,64 & 2,6 & 3,4 & 0,60 & 1,6 & $<4,4$ & 7,9 \\
\hline XIV-13 & 164,96 & 2,84 & 2,6 & 3,7 & 0,69 & 1,8 & $<4,4$ & 9,2 \\
\hline XIV-14 & 172,03 & 2,59 & 2,6 & 3,8 & 0,66 & 1,4 & $<4,4$ & 8,2 \\
\hline Rate Im & & & & & $47 \cdot 10^{-9}$ & $13 \cdot 10^{-8}$ & & $0^{-8}$ \\
\hline Rate [mo & & & & $2,1 \cdot 10^{\circ}$ & $4,1 \cdot 10^{\prime}$ & $1,3 \bullet 10^{\circ}$ & $*$ & \\
\hline
\end{tabular}




\section{Serizit}

Tab. A-32: Batch-Experimente mit Serizit Versuche 21 und 24.

21

Serizit

$\varnothing=6,3-20 \mu \mathrm{m}$

Einwaage Mineral [g]: 0,1503

Einwaage Lösung [g]: 100,0150

Lösung: $\mathbf{H}_{2} \mathrm{SO}_{4}, \mathbf{p H}=\mathbf{1}$

\begin{tabular}{|c|c|c|c|c|c|c|c|}
\hline \multirow[b]{2}{*}{ Probe } & \multirow[b]{2}{*}{ Tage } & \multirow[b]{2}{*}{$\mathbf{p H}$} & \multicolumn{5}{|l|}{$\mu \mathrm{mol} / 1$} \\
\hline & & & Al & $\mathbf{F e}$ & $\mathbf{K}$ & Mg & $\mathbf{S}$ i \\
\hline $21-1$ & 33,92 & 0,9 & 18 & 7,3 & 18 & 12 & 18 \\
\hline $21-2$ & 74,99 & 1,1 & 33 & 10 & 20 & 15 & 38 \\
\hline $21-3$ & 116,85 & 0,9 & 37 & 10 & 22 & 16 & 47 \\
\hline $21-4$ & 131,80 & 0,9 & 39 & 12 & 24 & 16 & 61 \\
\hline $21-5$ & 273,91 & 1,1 & 51 & 14 & 29 & 21 & 81 \\
\hline $21-6$ & 349,71 & 1,0 & 78 & 17 & 37 & 27 & 153 \\
\hline $21-7$ & 471,90 & 0,9 & 98 & 25 & 50 & 42 & 190 \\
\hline \multicolumn{2}{|c|}{ Rate $[\mathrm{mol} /(\mathrm{g} \bullet \mathrm{h})]$} & & $4,7 \cdot 10^{-9}$ & $9,8 \cdot 10^{-10}$ & $1,9 \cdot 10^{-9}$ & $1,7 \cdot 10^{-9}$ & $1,1 \cdot 10^{-8}$ \\
\hline
\end{tabular}

24

Serizit

$\varnothing=6,3-20 \mu \mathrm{m}$

Einwaage Mineral [g]: 0,1502

Einwaage Lösung [g]: 100,0000

Lösung: $\mathbf{H}_{2} \mathrm{SO}_{4}, \mathbf{p H}=\mathbf{2}$

\begin{tabular}{|c|c|c|c|c|c|c|c|}
\hline \multirow[b]{2}{*}{ Probe } & \multirow[b]{2}{*}{ Tage } & \multirow[b]{2}{*}{$\mathbf{p H}$} & \multicolumn{5}{|l|}{$\mu \mathrm{mol} / 1$} \\
\hline & & & Al & $\mathbf{F e}$ & $\mathbf{K}$ & Mg & $\mathbf{S}$ i \\
\hline $24-1$ & 33,98 & 1,8 & $\overline{7,8}$ & $\overline{5,5}$ & $\overline{\overline{6,1}}$ & 9,1 & $\overline{7,9}$ \\
\hline $24-2$ & 75,00 & 2,0 & 13 & 7,5 & 9,7 & 10 & 13 \\
\hline $24-3$ & 116,88 & 1,8 & 14 & 7,6 & 10 & 9,7 & 15 \\
\hline $24-4$ & 131,81 & 1,8 & 14 & 8,5 & 11 & 9,6 & 18 \\
\hline $24-5$ & 273,96 & 2,0 & 19 & 13 & 15 & 16 & 29 \\
\hline $24-6$ & 349,71 & 1,9 & 21 & 13 & 17 & 17 & 37 \\
\hline \multicolumn{2}{|c|}{ Rate $[\mathrm{mol} /(\mathrm{g} \bullet \mathrm{h})]$} & & $1,0 \bullet 10^{-9}$ & $6,9 \cdot 10^{-10}$ & $8,8 \cdot 10^{-10}$ & $7,8 \cdot 10^{-10}$ & $2,5 \cdot 10^{-9}$ \\
\hline
\end{tabular}


Tab. A-33: Batch-Experimente mit Serizit Versuche 44 und 50.

\section{4}

\section{Serizit}

$\emptyset=6,3-20 \mu \mathrm{m}$

Einwaage Mineral [g]: 0,1027

Einwaage Lösung [g]: 40,0083

Lösung: abgepreßte Porenlösung Solling

$$
\begin{aligned}
& \text { 0-15 cm Tiefe } \\
& \text { pH }=3,5
\end{aligned}
$$

DOC 39,15 mg/l

\begin{tabular}{|c|r|r|r|r|r|r|r|}
\hline & \multicolumn{9}{|c|}{$\mu \mathrm{mol} / 1$} \\
Probe & \multicolumn{1}{|c|}{ Tage } & \multicolumn{1}{c|}{$\mathbf{p H}$} & \multicolumn{1}{c|}{ A l } & \multicolumn{1}{c|}{ F e } & \multicolumn{1}{c|}{ K } & M g & \multicolumn{1}{c|}{ S i } \\
\hline \hline IN & 0 & 3,5 & 20 & 6,3 & 58 & 59 & 362 \\
\hline $44-1$ & 24,94 & 5,0 & 14 & 5,0 & 61 & 63 & 386 \\
\hline $44-2$ & 62,01 & 5,2 & 9,6 & 4,2 & 65 & 65 & 426 \\
\hline $44-3$ & 184,02 & 5,4 & 4,9 & 3,9 & 82 & 89 & 429 \\
\hline
\end{tabular}

\section{0}

\section{Serizit}

$\emptyset=6,3-20 \mu \mathrm{m}$

Einwaage Mineral [g]: 0,1553

Einwaage Lösung [g]: 50,0099

Lösung: $\mathrm{H}_{2} \mathrm{O}^{* * * * *}, \mathbf{p H}>\mathbf{4}$

\begin{tabular}{|l|r|r|r|r|r|r|r|}
\hline \multicolumn{1}{|c|}{ Probe } & \multicolumn{1}{c|}{ Tage } & \multicolumn{1}{l|}{ pH } & \multicolumn{1}{c|}{ A l } & \multicolumn{1}{c|}{ Fe } & \multicolumn{1}{c|}{ K } & \multicolumn{1}{c|}{ M g } & \multicolumn{1}{c|}{ S i } \\
\hline \hline $50-1$ & 25,00 & 4,8 & 1,1 & 0,72 & $<2,6$ & 7,8 & 3,1 \\
\hline $50-2$ & 61,87 & 4,9 & 0,68 & 0,25 & $<2,6$ & 11 & 8,1 \\
\hline $50-3$ & 115,01 & 5,5 & 2,5 & 4,0 & $<2,6$ & n.b. & 19 \\
\hline & & & & & & & \\
\hline
\end{tabular}


Tab. A-34: Batch-Experimente mit Serizit Versuche 51 und 52.

\section{1}

\section{Serizit}

$\emptyset=6,3-20 \mu \mathrm{m}$

Einwaage Mineral [g]: 0,1027

Einwaage Lösung [g]: 50,0093

Lösung: $\mathrm{H}_{2} \mathrm{SO}_{4}, \mathbf{p H}=\mathbf{3}$

\begin{tabular}{|c|c|c|c|c|c|c|c|}
\hline \multirow[b]{2}{*}{ Probe } & \multirow[b]{2}{*}{ Tage } & \multirow[b]{2}{*}{ pH } & \multicolumn{5}{|l|}{$\mu \mathrm{mol} / 1$} \\
\hline & & & Al & $\mathbf{F e}$ & $\mathbf{K}$ & Mg & $\mathbf{S ~ i ~}$ \\
\hline$\overline{51-1}$ & 24,99 & 3,1 & 7,2 & $\overline{5,1}$ & $<2,6$ & $\overline{4,2}$ & 7,1 \\
\hline $51-2$ & 61,85 & 3,1 & 8,7 & 6,8 & 15 & 9,4 & 13 \\
\hline $51-3$ & 115,00 & 3,2 & 12 & 11 & 31 & n.b. & 22 \\
\hline \multicolumn{2}{|c|}{ Rate $[\mathrm{mol} /(\mathrm{g} \bullet \mathrm{h})]$} & & $1,1 \cdot 10^{-9}$ & $1,3 \cdot 10^{-9}$ & $6,1 \cdot 10^{-9}$ & $2,9 \cdot 10^{-9}$ & $3,4 \cdot 10^{-9}$ \\
\hline
\end{tabular}

\section{2}

\section{Serizit}

$\emptyset=6,3-20 \mu \mathrm{m}$

Einwaage Mineral [g]: 0,1044

Einwaage Lösung [g]: 50,5618

Lösung: $\mathrm{HNO}_{3}, \mathbf{p H}=\mathbf{1}$

\begin{tabular}{|c|c|c|c|c|c|c|c|}
\hline & & & $\mu \mathrm{mol} / 1$ & & & & \\
\hline Probe & Tage & pH & Al & $\mathbf{F e}$ & $\mathbf{K}$ & Mg & $\mathbf{S} \mathbf{i}$ \\
\hline $52-1$ & 24,97 & 1,1 & 18 & 9,8 & 8,6 & 19 & 30 \\
\hline $52-2$ & 61,83 & 1,1 & 34 & 14 & 17 & 26 & 69 \\
\hline $52-3$ & 114,98 & 1,1 & 61 & 21 & 28 & n.b. & 90 \\
\hline $52-4$ & 184,00 & 1,1 & 64 & 27 & 33 & 43 & 140 \\
\hline \multicolumn{2}{|c|}{ Rate $[\mathrm{mol} /(\mathrm{g} \bullet \mathrm{h})]$} & & $6,0 \cdot 10^{-9}$ & $2,2 \cdot 10^{-9}$ & $3,1 \cdot 10^{-9}$ & $3,0 \cdot 10^{-9}$ & $1,3 \cdot 10^{-8}$ \\
\hline
\end{tabular}


Tab. A-35: Batch-Experimente mit Serizit Versuche 53 und 54.

\section{3}

\section{Serizit}

$\varnothing=6,3-20 \mu \mathrm{m}$

Einwaage Mineral [g]: 0,1037

Einwaage Lösung [g]: 50,1676

Lösung: $\mathbf{H N O}_{3}, \mathbf{p H}=\mathbf{2}$

\begin{tabular}{|l|r|r|r|r|r|r|r|}
\hline \multicolumn{1}{|c|}{ Probe } & Tage & \multicolumn{1}{c|}{ pH } & \multicolumn{1}{c|}{ A l } & \multicolumn{1}{c|}{ F e } & \multicolumn{1}{c|}{ K } & \multicolumn{1}{c|}{ M g } & \multicolumn{1}{c|}{$\mathbf{S ~ i}$} \\
\hline \hline $53-1$ & 24,97 & 2,0 & 10 & 7,2 & $<2,6$ & 8,4 & 14 \\
\hline $53-2$ & 61,83 & 2,1 & 14 & 11 & 5,9 & 12 & 22 \\
\hline $53-3$ & 114,98 & 2,0 & 21 & 17 & 12 & n.b. & 28 \\
\hline \multicolumn{2}{l|l}{ Rate $[\mathrm{mol} /(\mathrm{g} \bullet \mathrm{h})]$} & & & & & & \\
\hline
\end{tabular}

\section{4}

\section{Serizit}

$\varnothing=6,3-20 \mu \mathrm{m}$

Einwaage Mineral [g]: 0,1057

Einwaage Lösung [g]: 50,4328

Lösung: $\mathrm{HNO}_{3}, \mathbf{p H}=\mathbf{3}$

\begin{tabular}{|c|c|c|c|c|c|c|c|}
\hline \multirow[b]{2}{*}{ Probe } & \multirow[b]{2}{*}{ Tage } & \multirow[b]{2}{*}{ pH } & \multicolumn{5}{|l|}{$\mu \mathrm{mol} / 1$} \\
\hline & & & Al & $\mathbf{F e}$ & $\mathbf{K}$ & Mg & $\mathbf{S} \mathbf{i}$ \\
\hline $54-1$ & 24,97 & 3,2 & 4,4 & 3,2 & $<2,6$ & 4,4 & 5,2 \\
\hline $54-2$ & 61,83 & 3,2 & 5,4 & 4,5 & $<2,6$ & 8,4 & 9,5 \\
\hline $54-3$ & 114,98 & 3,2 & 8,5 & 8,5 & 5,0 & n.b. & 16 \\
\hline \multicolumn{2}{|c|}{ Rate $[\mathrm{mol} /(\mathrm{g} \bullet \mathrm{h})]$} & & $9,2 \cdot 10^{-10}$ & $1,2 \cdot 10^{-9}$ & & $2,2 \cdot 10^{-9}$ & $2,4 \cdot 10^{-9}$ \\
\hline
\end{tabular}


Tab. A-36: Batch-Experimente mit Serizit Versuch 55.

\section{5}

\section{Serizit}

$\varnothing=6,3-20 \mu \mathrm{m}$

Einwaage Mineral [g]: 0,1069

Einwaage Lösung [g]: 50,0368

Lösung: $\mathbf{C}_{6} \mathbf{H}_{8} \mathbf{O}_{7} \cdot \mathbf{H}_{2} \mathbf{O}, \mathbf{M}=\mathbf{0 , 0 1}$

\begin{tabular}{|l|r|r|r|r|r|r|r|}
\hline \multicolumn{1}{|c|}{ Probe } & Tage & \multicolumn{1}{c|}{ pH } & \multicolumn{1}{c|}{ A l } & \multicolumn{1}{c|}{ Fe } & \multicolumn{1}{c|}{ K } & Mg & \multicolumn{1}{c|}{ S i } \\
\hline \hline $55-1$ & 24,99 & 2,6 & 9,4 & 7,2 & $<2,6$ & 5,2 & 11 \\
\hline $55-2$ & 61,85 & 2,6 & 14 & 8,4 & $<2,6$ & 6,8 & 20 \\
\hline $55-3$ & 115,00 & 2,8 & 22 & 12 & $<2,6$ & n.b. & 27 \\
\hline \multicolumn{2}{l|l}{ Rate $[\mathrm{mol} /(\mathrm{g} \bullet \mathrm{h})]$} & & & & & & \\
\hline
\end{tabular}


Tab. A-37: Durchfluß-Experimente mit Serizit Versuche IX(1)und IX(2).

\section{IX(1)}

\section{Serizit}

$\varnothing=6,3-20 \mu \mathrm{m}$

Einwaage Mineral [g]: 0,7799

Einwaage Lösung [g]: 246,2

Lösung: $\mathrm{H}_{2} \mathrm{SO}_{4}, \mathbf{p H}=1$

\begin{tabular}{|c|c|c|c|c|c|c|c|c|}
\hline & & & & $\mu \mathrm{mol} / 1$ & & & & \\
\hline Probe & Tage & g/h & $\mathbf{p H}$ & Al & $\mathbf{F e}$ & $\mathbf{K}$ & $\mathrm{Mg}$ & $\mathbf{S i}$ \\
\hline IX(1)-1 & 7,06 & 1,81 & 1,4 & 27 & 11 & 16 & 15 & 27 \\
\hline IX(1)-2 & 8,28 & 1,67 & 1,4 & 27 & 10 & 16 & 14 & 27 \\
\hline IX(1)-3 & 26,28 & 1,02 & 1,2 & 26 & 2,7 & 15 & 5,7 & 26 \\
\hline IX(1)-4 & 40,08 & 2,40 & 1,2 & 20 & 1,5 & 13 & 3,6 & 21 \\
\hline IX(1)-5 & 46,08 & 2,43 & 1,2 & 16 & 1,3 & 11 & 3,1 & 20 \\
\hline IX(1)-6 & 48,10 & 2,09 & 1,2 & 16 & 1,2 & 9,9 & 2,9 & 19 \\
\hline IX(1)-7 & 50,97 & 2,82 & 1,1 & 16 & 1,3 & 8,9 & 2,8 & 22 \\
\hline IX(1)-8 & 54,17 & 2,34 & 1,1 & 16 & 1,2 & 8,8 & 2,8 & 23 \\
\hline IX(1)-9 & 58,18 & 2,27 & 1,1 & 16 & 1,2 & 8,4 & 2,7 & 23 \\
\hline IX(1)-10 & 62,10 & 2,29 & 1,1 & 15 & 1,1 & 8,2 & 2,5 & 23 \\
\hline IX(1)-11 & 65,08 & 2,32 & 1,1 & 15 & 1,1 & 7,9 & 2,4 & 22 \\
\hline IX(1)-12 & 68,13 & 2,29 & 1,1 & 14 & 1,1 & 7,9 & 2,4 & 22 \\
\hline IX(1)-13 & 75,10 & 2,51 & 1,1 & 13 & 0,94 & 7,1 & 2,1 & 21 \\
\hline IX(1)-14 & 83,10 & 2,43 & 1,2 & 15 & 0,87 & 7,6 & $<0,82$ & 20 \\
\hline \multicolumn{2}{|c|}{ Rate $[\mathrm{mol} /(\mathrm{g} \bullet \mathrm{h})]$} & & & $4,4 \cdot 10^{-8}$ & $3,1 \cdot 10^{-9}$ & $2,3 \cdot 10^{-8}$ & $7,0 \bullet 10^{-9}$ & $6,5 \cdot 10^{-8}$ \\
\hline
\end{tabular}

\section{IX(2)}

\section{Serizit}

$\varnothing=6,3-20 \mu \mathrm{m}$

Einwaage Mineral [g]: 0,7799

Einwaage Lösung [g]: 246,2

Lösung: $\mathbf{H}_{2} \mathrm{SO}_{4}, \mathbf{p H}=\mathbf{2}$

\begin{tabular}{|c|c|c|c|c|c|c|c|c|}
\hline & & & & $\mu \mathrm{mol} / 1$ & & & & \\
\hline Probe & Tage & $\mathrm{g} / \mathrm{h}$ & $\mathrm{pH}$ & $\overline{A l}$ & $\mathbf{F e}$ & $\mathbf{K}$ & Mg & $\mathbf{S i}$ \\
\hline IX(2)-1 & 7,50 & 2,93 & 2,1 & 3,6 & $<0,18$ & $\overline{<2,6}$ & 0,85 & $\overline{6,9}$ \\
\hline IX(2)-2 & 12,53 & 2,75 & 2,0 & 5,2 & $<0,18$ & $<2,6$ & 0,91 & 10 \\
\hline IX(2)-3 & 19,63 & 2,28 & 2,0 & 5,6 & 0,53 & 3,5 & 1,5 & 13 \\
\hline IX(2)-4 & 26,65 & 2,79 & 2,0 & 5,2 & $<0,18$ & $<2,6$ & 3,8 & 14 \\
\hline IX(2)-5 & 33,55 & 2,81 & 1,9 & 5,3 & $<0,18$ & $<2,6$ & 3,9 & 14 \\
\hline IX(2)-6 & 40,57 & 2,79 & 1,9 & 5,5 & $<0,18$ & $<2,6$ & 3,9 & 14 \\
\hline IX(2)-7 & 47,54 & 2,87 & 2,0 & 6,4 & $<0,18$ & $<2,6$ & 4,0 & 15 \\
\hline \multicolumn{2}{|c|}{ Rate $[\mathrm{mol} /(\mathrm{g} \bullet \mathrm{h})]$} & & & $2,0 \cdot 10^{-8}$ & & & $1,4 \cdot 10^{-8}$ & $5,1 \bullet 10^{-8}$ \\
\hline
\end{tabular}


Tab. A-38: Durchfluß-Experimente mit Serizit Versuch XV.

\section{XV}

\section{Serizit}

$\varnothing=6,3-20 \mu \mathrm{m}$

Einwaage Mineral [g]: 0,3501

Einwaage Lösung [g]: 86,3

Lösung: $\mathrm{C}_{6} \mathrm{H}_{8} \mathrm{O}_{7} \bullet \mathrm{H}_{2} \mathrm{O}, \mathrm{M}=\mathbf{0 , 0 1}$

\begin{tabular}{|c|c|c|c|c|c|c|c|c|}
\hline & & & & $\mu \mathrm{mol} / \mathrm{l}$ & & & & \\
\hline Probe & Tage & $\mathrm{g} / \mathrm{h}$ & pH & $\overline{A l}$ & $\mathbf{F e}$ & $K$ & Mg & $\mathbf{S i}$ \\
\hline XV-1 & 13,04 & 2,95 & 2,6 & 4,5 & 0,91 & $<2,6$ & 4,4 & 10 \\
\hline $\mathrm{XV}-2$ & 17,94 & 2,68 & 2,6 & 1,4 & $<0,18$ & $<2,6$ & 1,0 & 2,8 \\
\hline XV-3 & 24,99 & 2,37 & 2,6 & 1,8 & 0,26 & $<2,6$ & 1,3 & 3,9 \\
\hline XV-4 & 32,06 & 2,92 & 2,7 & 2,5 & $<0,18$ & $<2,6$ & 3,9 & 5,1 \\
\hline XV-5 & 38,96 & 2,82 & 2,7 & 2,6 & $<0,18$ & $<2,6$ & 3,8 & 5,3 \\
\hline XV-6 & 45,97 & 2,40 & 2,7 & 2,9 & $<0,18$ & $<2,6$ & 3,8 & 5,1 \\
\hline XV-7 & 52,95 & 2,57 & 2,6 & 2,6 & $<0,18$ & $<2,6$ & 3,6 & 5,2 \\
\hline XV-8 & 118,19 & 1,77 & 2,6 & 1,6 & 0,30 & 2,8 & 1,5 & 2,1 \\
\hline XV-9 & 127,05 & 2,84 & 2,6 & 1,3 & 0,31 & $<2,6$ & 0,84 & 1,7 \\
\hline $\mathrm{XV}-10$ & 131,01 & 2,79 & 2,6 & 1,3 & 0,25 & $<2,6$ & $<0,82$ & 1,5 \\
\hline $\mathrm{XV}-11$ & 144,08 & 2,27 & 2,6 & 1,4 & 0,27 & 2,7 & 0,87 & 1,2 \\
\hline $\mathrm{XV}-12$ & 150,98 & 2,92 & 2,5 & 1,3 & 0,23 & $<2,6$ & 0,96 & 1,1 \\
\hline$X V-13$ & 159,05 & 2,07 & 2,6 & 1,4 & 0,29 & 3,3 & 1,0 & 1,1 \\
\hline $\mathrm{XV}-14$ & 164,95 & 2,86 & 2,6 & 1,3 & 0,24 & $<2,6$ & 1,3 & 1,1 \\
\hline $\mathrm{XV}-15$ & 172,02 & 2,61 & 2,6 & 1,4 & 0,27 & 2,7 & 0,99 & 1,2 \\
\hline \multicolumn{2}{|c|}{ Rate $[\mathrm{mol} /(\mathrm{g} \bullet \mathrm{h})]$} & & & $9,9 \cdot 10^{-9}$ & $1,9 \cdot 10^{-9}$ & $2,1 \cdot 10^{-8}$ & $7,5 \cdot 10^{-9}$ & $8,3 \cdot 10^{-9}$ \\
\hline
\end{tabular}




\section{Illit H-35}

Tab. A-39: Batch-Experimente mit Illit H-35 Versuche 10 und 11.

\section{0}

\section{Illit $\mathbf{H}-35$}

$\varnothing=0,63-2 \mu \mathrm{m}$

Einwaage Mineral [g]: 0,1506

Einwaage Lösung [g]: 100,2539

Lösung: $\mathbf{H}_{2} \mathrm{SO}_{4}, \mathbf{p H}=\mathbf{1}$

\begin{tabular}{|c|c|c|c|c|c|c|c|}
\hline \multirow[b]{2}{*}{ Probe } & \multirow[b]{2}{*}{ Tage } & \multicolumn{3}{|c|}{$\mu \mathrm{mol} / 1$} & \multirow[b]{2}{*}{$\boldsymbol{K}$} & \multirow[b]{2}{*}{ Mg } & \multirow[b]{2}{*}{$S$ i } \\
\hline & & pH & Al & $\mathbf{F e}$ & & & \\
\hline $10-1$ & 19,21 & 1,2 & 388 & 765 & 75 & 287 & 468 \\
\hline $10-2$ & 100,09 & 1,0 & 445 & 469 & 105 & 310 & 608 \\
\hline $10-3$ & 184,04 & 1,0 & 584 & 511 & 142 & 313 & 800 \\
\hline $10-4$ & 380,02 & 1,1 & 807 & 529 & 186 & 327 & 1.262 \\
\hline $10-5$ & 416 , & 1,0 & 844 & 591 & 188 & 351 & 1.561 \\
\hline $10-6$ & 538,98 & 1,0 & 963 & 776 & 301 & 484 & 1.819 \\
\hline Rate [mo & & & $3,2 \cdot 10^{-8}$ & $3,6 \cdot 10^{-9}$ & $1,0 \cdot 10^{-8}$ & $8,1 \cdot 10^{-9}$ & $7,4 \cdot 10^{-8}$ \\
\hline
\end{tabular}

11

Illit H-35

$\varnothing=0,63-2 \mu \mathrm{m}$

Einwaage Mineral [g]: 0,1507

Einwaage Lösung [g]: 100,2522

Lösung: $\mathbf{H}_{2} \mathrm{SO}_{4}, \mathbf{p H}=\mathbf{2}$

\begin{tabular}{|c|c|c|c|c|c|c|c|}
\hline \multirow[b]{2}{*}{ Probe } & \multirow[b]{2}{*}{ Tage } & \multirow[b]{2}{*}{$\mathbf{p H}$} & \multicolumn{5}{|l|}{$\mu \mathrm{mol} / 1$} \\
\hline & & & Al & $\mathbf{F e}$ & $\mathbf{K}$ & Mg & $\mathbf{S} \mathbf{i}$ \\
\hline $11-1$ & 19,11 & 2,1 & 157 & 297 & 16 & 141 & 194 \\
\hline $11-2$ & 40,06 & 1,9 & 229 & 531 & 33 & 217 & 286 \\
\hline $11-3$ & 99,99 & 1,9 & 282 & 436 & 48 & 271 & 352 \\
\hline $11-4$ & 183,94 & 1,9 & 345 & 491 & 60 & 280 & 426 \\
\hline $11-5$ & 379,92 & 2,0 & 378 & 529 & 65 & 302 & 566 \\
\hline $11-6$ & 415,96 & 2,0 & 382 & 542 & 65 & 298 & 628 \\
\hline $11-7$ & 538,88 & 2,0 & 385 & 695 & 89 & 402 & 664 \\
\hline \multicolumn{2}{|c|}{ Rate $[\mathrm{mol} /(\mathrm{g} \bullet \mathrm{h})]$} & & $1,1 \cdot 10^{-8}$ & $1,3 \cdot 10^{-8}$ & $3,0 \cdot 10^{-9}$ & $9,6 \cdot 10^{-9}$ & $2,4 \cdot 10^{-8}$ \\
\hline
\end{tabular}


Tab. A-40: Batch-Experimente mit Illit H-35 Versuche 25 und 28.

25

Illit $\mathbf{H}-\mathbf{3 5}$

$\varnothing=0,63-2 \mu \mathrm{m}$

Einwaage Mineral [g]: 0,1506

Einwaage Lösung [g]: 100,0120

Lösung: $\mathrm{C}_{6} \mathrm{H}_{8} \mathrm{O}_{7} \cdot \mathbf{H}_{2} \mathrm{O}, \mathbf{M}=\mathbf{0 , 0 1}$

\begin{tabular}{|c|c|c|c|c|c|c|c|}
\hline & & & \multicolumn{5}{|l|}{$\mu \mathrm{mol} / \mathrm{l}$} \\
\hline Probe & Tage & $\mathrm{pH}$ & Al & $\mathbf{F e}$ & $\mathrm{K}$ & $\mathrm{Mg}$ & $\mathbf{S i}$ \\
\hline$\overline{25-1}$ & $\overline{8,08}$ & 2,5 & 114 & 233 & n.b. & 95 & 136 \\
\hline $25-2$ & 29,01 & 2,5 & 211 & 519 & 43 & 175 & 257 \\
\hline $25-3$ & 89,99 & 2,6 & 298 & 388 & 57 & 196 & 287 \\
\hline $25-4$ & 172,90 & 3,1 & 334 & 450 & 55 & 254 & 419 \\
\hline $25-5$ & 187,83 & 3,0 & 339 & 487 & 54 & 249 & 490 \\
\hline $25-6$ & 330,01 & 3,5 & 149 & 82 & 51 & 106 & 355 \\
\hline $25-7$ & 368,86 & 3,3 & 64 & 49 & 39 & 224 & 395 \\
\hline $25-8$ & 404,99 & 5,6 & 4,7 & 2,0 & 41 & 225 & 402 \\
\hline $25-9$ & 528,00 & 5,2 & 0,45 & 0,67 & 55 & 303 & 363 \\
\hline Rate [mo & & & $3,0 \cdot 10^{-8}$ & $1.9 \cdot 10^{-8}$ & $1.6 \cdot 10^{-9}$ & $2,0 \cdot 10^{-8}$ & $4,6 \cdot 10^{-8}$ \\
\hline
\end{tabular}

28

Illit H-35

$\varnothing=0,63-2 \mu \mathrm{m}$

Einwaage Mineral [g]: 0,1489

Einwaage Lösung [g]: 100,0034

Lösung: $\mathrm{H}_{2} \mathrm{SO}_{4}, \mathbf{p H}=\mathbf{3}$

\begin{tabular}{|c|c|c|c|c|c|c|c|}
\hline & & & $\mu \mathrm{mol} / 1$ & & & & \\
\hline Probe & Tage & pH & A l & $\mathbf{F e}$ & $\mathbf{K}$ & Mg & $\mathbf{S}$ i \\
\hline $28-1$ & 2,22 & 4,0 & 2,8 & 0,45 & 7,5 & 41 & 25 \\
\hline $28-2$ & 17,13 & 3,7 & 4,0 & 14 & 8,2 & 46 & 45 \\
\hline $28-3$ & 159,32 & 3,4 & 6,8 & 42 & 20 & 73 & 58 \\
\hline $28-4$ & 234,28 & 3,5 & 9,2 & 37 & 17 & 66 & 73 \\
\hline $28-5$ & 286,02 & 3,7 & 14 & 39 & 26 & 102 & 87 \\
\hline \multicolumn{2}{|c|}{ Rate $[\mathrm{mol} /(\mathrm{g} \bullet \mathrm{h})]$} & & $9,4 \cdot 10^{-10}$ & $3,6 \cdot 10^{-9}$ & $1,6 \cdot 10^{-9}$ & $4,9 \cdot 10^{-9}$ & $5,1 \cdot 10^{-9}$ \\
\hline
\end{tabular}


Tab. A-41: Batch-Experimente mit Illit H-35 Versuche 31 und 32.

31

Illit $\mathbf{H}-35$

$\varnothing=0,63-2 \mu \mathrm{m}$

Einwaage Mineral [g]: 0,1500

Einwaage Lösung [g]: 100,0049

Lösung: $\mathrm{HNO}_{3}, \mathbf{p H}=\mathbf{1}$

\begin{tabular}{|l|r|r|r|r|r|r|r|}
\hline \multicolumn{1}{|c|}{ Probe } & Tage & \multicolumn{1}{|c|}{ pH } & \multicolumn{1}{|c|}{ A l } & \multicolumn{1}{c|}{ Fe } & \multicolumn{1}{|c|}{ K } & \multicolumn{1}{c|}{ Mg } & \multicolumn{1}{c|}{ S i } \\
\hline $31-1$ & 2,23 & 1,0 & 102 & 191 & 34 & 71 & 99 \\
\hline $31-2$ & 17,14 & 1,1 & 243 & 398 & 66 & 167 & 332 \\
\hline $31-3$ & 198,17 & 1,2 & 852 & 446 & 195 & 271 & 1.095 \\
\hline $31-4$ & 235,02 & 1,1 & 888 & 602 & 199 & 352 & 1.599 \\
\hline $31-5$ & 357,24 & 1,0 & 925 & 805 & 279 & 489 & 1.736 \\
\hline & & & & & & & \\
\hline
\end{tabular}

\section{2}

\section{Illit $\mathbf{H}-35$}

$\varnothing=0,63-2 \mu \mathrm{m}$

Einwaage Mineral [g]: 0,1500

Einwaage Lösung [g]: 100,0038

Lösung: $\mathrm{HNO}_{3}, \mathbf{p H}=\mathbf{2}$

\begin{tabular}{|l|r|r|r|r|r|r|r|}
\hline \multicolumn{1}{|c|}{ Probe } & Tage & \multicolumn{1}{c|}{ pH } & \multicolumn{1}{c|}{ A l } & \multicolumn{1}{c|}{ F e } & \multicolumn{1}{c|}{ K } & \multicolumn{1}{c|}{ M g } & \multicolumn{1}{c|}{ S i } \\
\hline \hline $32-1$ & 2,23 & 2,0 & 49 & 55 & 13 & 59 & 57 \\
\hline $32-2$ & 17,14 & 2,1 & 92 & 201 & 14 & 80 & 137 \\
\hline $32-3$ & 159,40 & 2,1 & 218 & 192 & 32 & 173 & 159 \\
\hline $32-4$ & 235,02 & 2,2 & 322 & 494 & 53 & 264 & 490 \\
\hline $32-5$ & 357,24 & 2,0 & 343 & 670 & 74 & 383 & 550 \\
\hline & & & & & & & \\
\hline & & & $2,4 \cdot 10^{-8}$ & $4,4 \cdot 10^{-8}$ & $4,8 \cdot 10^{-9}$ & $2,5 \cdot 10^{-8}$ & $3,9 \cdot 10^{-8}$ \\
\hline
\end{tabular}


Tab. A-42: Batch-Experimente mit Illit H-35 Versuche 33 und 46. IN kenzeichnet die InputLösung.

33

Illit $\mathbf{H 3 5}$

$\emptyset=0,63-2 \mu \mathrm{m}$

Einwaage Mineral [g]: 0,1483

Einwaage Lösung [g]: 100,0070

Lösung: $\mathrm{HNO}_{3}, \mathbf{p H}=\mathbf{3}$

\begin{tabular}{|c|c|c|c|c|c|c|c|}
\hline \multirow[b]{2}{*}{ Probe } & \multirow[b]{2}{*}{ Tage } & \multirow[b]{2}{*}{$\mathbf{p H}$} & \multicolumn{5}{|l|}{$\mu \mathrm{mol} / 1$} \\
\hline & & & A l & $\mathbf{F e}$ & $\mathbf{K}$ & Mg & $\mathbf{S}$ i \\
\hline $33-1$ & 2,23 & 3,9 & 2,5 & 0,27 & 6,6 & 40 & 23 \\
\hline $33-2$ & 17,14 & 3,9 & 3,4 & 8,7 & 8,5 & 43 & 40 \\
\hline $33-3$ & 159,40 & 3,2 & 5,3 & 33 & 17 & 66 & 52 \\
\hline $33-4$ & 235,02 & 3,7 & 7,8 & 33 & 17 & 62 & 68 \\
\hline $33-5$ & 286,03 & 3,6 & 13 & 43 & 26 & 95 & 79 \\
\hline $33-6$ & 357,24 & 3,3 & 11 & 54 & 27 & 91 & 80 \\
\hline Rate [mol/ & & & $7,6 \cdot 10^{-10}$ & $3,9 \cdot 10^{-9}$ & $1,6 \cdot 10^{-9}$ & $4,2 \cdot 10^{-9}$ & $4,3 \cdot 10^{-9}$ \\
\hline
\end{tabular}

46

Illit $\mathbf{H 3 5}$

$\varnothing=0,63-2 \mu \mathrm{m}$

Einwaage Mineral [g]: 0,0613

Einwaage Lösung [g]: 39,7198

Lösung: abgepreßte Porenlösung Solling 0-15 cm Tiefe

pH $=3,5$

DOC $=39,15 \mathrm{mg} / \mathrm{l}$

\begin{tabular}{|c|r|r|r|r|r|r|r|}
\hline & \multicolumn{2}{|c|}{$\mu \mathrm{mol} / 1$} \\
Probe & \multicolumn{1}{|c|}{ Tage } & \multicolumn{1}{c|}{$\mathbf{p H}$} & \multicolumn{1}{c|}{ A l } & \multicolumn{1}{c|}{ F e } & \multicolumn{1}{c|}{ K } & \multicolumn{1}{c|}{ M g } & \multicolumn{1}{c|}{ S i } \\
\hline \hline IN & 0 & 3,5 & 20 & 6,3 & 58 & 59 & 362 \\
\hline $46-1$ & 24,94 & 5,2 & 6,0 & 1,1 & 54 & 73 & 387 \\
\hline $46-2$ & 62,01 & 5,5 & 4,4 & 1,3 & 60 & 76 & 432 \\
\hline $46-3$ & 184,02 & 5,7 & 1,2 & 1,3 & 79 & 105 & 422 \\
\hline
\end{tabular}


Tab. A-43: Durchfluß-Experimente mit Illit H-35 Versuch VII(1).

\section{VII(1)}

\section{Illit $\mathbf{H}-\mathbf{3 5}$}

$\varnothing=0,63-2 \mu \mathrm{m}$

Einwaage Mineral [g]: 0,7204

Einwaage Lösung [g]: 249,8

Lösung: $\mathrm{H}_{2} \mathrm{SO}_{4}, \mathbf{p H}=\mathbf{1}$

\begin{tabular}{|c|c|c|c|c|c|c|c|c|}
\hline & & & & $\mu \mathrm{mol} / 1$ & & & & \\
\hline Probe & Tage & g/h & pH & Al & $\mathbf{F e}$ & $\mathbf{K}$ & $\mathrm{Mg}$ & $\mathbf{S i}$ \\
\hline VII(1)-1 & 3,20 & 1,75 & 1,4 & 315 & 410 & 54 & 249 & 374 \\
\hline VII(1)-2 & 6,10 & 0,93 & 1,4 & 317 & 380 & 57 & 249 & 396 \\
\hline VII(1)-3 & 18,24 & 1,79 & 1,4 & 176 & 184 & 40 & 122 & 253 \\
\hline VII(1)-4 & 19,18 & 1,33 & 1,3 & 154 & 169 & 38 & 112 & 226 \\
\hline VII(1)-5 & 31,28 & 0,85 & 1,2 & 108 & 74 & 41 & 44 & 177 \\
\hline VII(1)-6 & 36,24 & 6,11 & 1,2 & 102 & 58 & 37 & 36 & 171 \\
\hline VII(1)-7 & 45,08 & 2,34 & 1,2 & 49 & 18 & 21 & 11 & 79 \\
\hline VII(1)-8 & 51,08 & 2,44 & 1,2 & 44 & 14 & 19 & 9,0 & 72 \\
\hline VII(1)-9 & 53,10 & 2,07 & 1,2 & 44 & 14 & 18 & 8,7 & 72 \\
\hline VII(1)-10 & 55,97 & 2,72 & 1,2 & 47 & 14 & 17 & 8,4 & 83 \\
\hline VII(1)-11 & 59,17 & 2,32 & 1,1 & 46 & 14 & 17 & 8,1 & 82 \\
\hline VII(1)-12 & 63,18 & 2,22 & 1,1 & 45 & 13 & 16 & 7,8 & 81 \\
\hline VII(1)-13 & 67,10 & 2,27 & 1,1 & 44 & 12 & 15 & 7,5 & 80 \\
\hline VII(1)-14 & 70,08 & 2,29 & 1,1 & 43 & 12 & 15 & 7,3 & 79 \\
\hline VII(1)-15 & 73,13 & 2,26 & 1,2 & 42 & 11 & 14 & 7,0 & 77 \\
\hline VII(1)-16 & 77,31 & 2,14 & 1,2 & 39 & 10 & 14 & 6,6 & 73 \\
\hline VII(1)-17 & 80,10 & 2,57 & 1,2 & 37 & 9,8 & 12 & 6,2 & 70 \\
\hline \multicolumn{2}{|c|}{ Rate $[\mathrm{mol} /(\mathrm{g} \bullet \mathrm{h})]$} & & & $1,4 \bullet 10^{-7}$ & $3,9 \cdot 10^{-8}$ & $4,9 \cdot 10^{-8}$ & $2,4 \cdot 10^{-8}$ & $2,5 \cdot 10^{-7}$ \\
\hline
\end{tabular}


Tab. A-44: Durchfluß-Experimente mit Illit H-35 Versuch VII(2).

\section{$\operatorname{VII}(2)$}

\section{Illit H-35}

$\emptyset=0,63-2 \mu \mathrm{m}$

Einwaage Mineral [g]: 0,7204

Einwaage Lösung [g]: 249,8

Lösung: $\mathbf{H}_{2} \mathrm{SO}_{4}, \mathbf{p H}=\mathbf{2}$

\begin{tabular}{|c|c|c|c|c|c|c|c|c|}
\hline & & & & $\mu \mathrm{mol} / 1$ & & & & \\
\hline Probe & Tage & g/h & pH & Al & $\mathbf{F e}$ & $\mathbf{K}$ & Mg & $\mathbf{S i}$ \\
\hline VIII(2)1 & 17,06 & 2,24 & 1,8 & 15 & 6,7 & 6,3 & $<0,82$ & 42 \\
\hline VII $(2) 2$ & 20,04 & 2,38 & 1,9 & 14 & 5,4 & 6,1 & $<0,82$ & 35 \\
\hline VII $(2) 3$ & 35,95 & 2,68 & 1,9 & 32 & 7,6 & 8,7 & $<0,82$ & 53 \\
\hline VII $(2) 4$ & 41,95 & 2,53 & 1,9 & 21 & 4,7 & 5,7 & $<0,82$ & 33 \\
\hline VII $(2) 5$ & 44,89 & 2,67 & 1,9 & 23 & 4,5 & 6,9 & $<0,82$ & 32 \\
\hline VII $(2) 6$ & 54,95 & 2,44 & 2,0 & 17 & 4,6 & 5,5 & $<0,82$ & 37 \\
\hline VII $(2) 7$ & 57,08 & 1,91 & 2,0 & 17 & 5,1 & 9,3 & $<0,82$ & 36 \\
\hline VIII $(2) 8$ & 58,97 & 2,45 & 2,0 & 17 & 4,3 & 5,2 & $<0,82$ & 35 \\
\hline VII $(2) 9$ & 61,93 & 2,47 & 1,9 & 15 & 3,7 & 4,7 & $<0,82$ & 31 \\
\hline VII(2)10 & 63,95 & 2,20 & 2,0 & 16 & 3,8 & 4,8 & 2,4 & 30 \\
\hline VII(2)11 & 66,08 & 1,68 & 2,0 & 17 & 4,5 & 7,3 & 3,1 & 33 \\
\hline VII(2)12 & 68,99 & 2,50 & 2,0 & 15 & 3,5 & 4,5 & 2,2 & 28 \\
\hline VII(2)13 & 75,99 & 2,41 & 2,0 & 14 & 3,3 & 4,4 & 2,1 & 27 \\
\hline VII(2)14 & 78,03 & 2,40 & 2,0 & 14 & 3,3 & 4,4 & 2,1 & 28 \\
\hline VII(2)15 & 78,97 & 2,49 & 2,0 & 13 & 3,3 & 4,4 & 2,1 & 27 \\
\hline VII(2)16 & 84,92 & 2,55 & 2,0 & 14 & 3,3 & 4,6 & 2,1 & 28 \\
\hline VII(2)17 & 86,92 & 2,60 & 2,0 & 15 & 3,6 & 5,0 & 2,4 & 31 \\
\hline VII(2)18 & 90,88 & 2,52 & 2,0 & 14 & 3,4 & 5,0 & 2,3 & 29 \\
\hline VII(2)19 & 92,86 & 2,58 & 2,0 & 14 & 3,3 & 4,6 & 2,3 & 29 \\
\hline VII(2)20 & 96,95 & 2,50 & 2,0 & 15 & 3,4 & 4,5 & 2,2 & 29 \\
\hline VII(2)21 & 99,01 & 2,53 & 2,0 & 15 & 3,3 & 4,4 & 2,1 & 28 \\
\hline VII(2)22 & 103,91 & 2,62 & 2,0 & 15 & 3,4 & 4,4 & 2,1 & 28 \\
\hline $\mathrm{VII}(2) 23$ & 105,97 & 2,62 & 2,0 & 16 & 3,0 & $<2,6$ & 2,2 & 34 \\
\hline VII(2)24 & 107,89 & 2,58 & 2,0 & 17 & 2,9 & $<2,6$ & 2,2 & 35 \\
\hline VII(2)25 & 112,94 & 2,53 & 2,0 & 16 & 2,8 & $<2,6$ & 2,1 & 35 \\
\hline VII(2)26 & 114,99 & 2,53 & 2,0 & 16 & 2,7 & $<2,6$ & 2,1 & 34 \\
\hline VII(2)27 & 117,96 & 2,64 & 2,0 & 16 & 2,8 & $<2,6$ & 2,1 & 34 \\
\hline VII(2)28 & 119,89 & 2,74 & 2,0 & 16 & 2,7 & $<2,6$ & 2,1 & 34 \\
\hline VII(2)29 & 122,03 & 2,20 & 2,0 & 17 & 2,8 & $<2,6$ & 2,2 & 33 \\
\hline VII(2)30 & 125,90 & 2,75 & 1,9 & 18 & 3,2 & $<2,6$ & 2,4 & 38 \\
\hline VII(2)31 & 128,01 & 2,69 & 1,9 & 18 & 3,0 & $<2,6$ & 2,3 & 35 \\
\hline VII(2)32 & 131,97 & 2,81 & 1,9 & 18 & 3,0 & $<2,6$ & 2,3 & 35 \\
\hline VII(2)33 & 135,05 & 2,68 & 1,9 & 18 & 3,0 & $<2,6$ & 2,1 & 35 \\
\hline VII(2)34 & 138,92 & 2,93 & 1,9 & 16 & 2,9 & $<2,6$ & 2,3 & 35 \\
\hline VII(2)35 & 141,13 & 2,62 & 2,0 & 17 & 3,7 & $<2,6$ & 2,4 & 39 \\
\hline VII(2)36 & 143,00 & 2,66 & 1,9 & 14 & 2,4 & $<2,6$ & 1,8 & 28 \\
\hline VII(2)37 & 146,92 & 2,63 & 1,9 & 17 & 2,7 & $<2,6$ & 2,0 & 32 \\
\hline VII(2)38 & 150,00 & 2,72 & 1,9 & 16 & 2,6 & $<2,6$ & 2,0 & 32 \\
\hline VII(2)39 & 152,89 & 2,70 & 2,0 & 16 & 2,6 & $<2,6$ & 2,0 & 33 \\
\hline \multicolumn{2}{|c|}{ Rate $[\mathrm{mol} /(\mathrm{g} \bullet \mathrm{h})]$} & & & $6,3 \cdot 10^{-8}$ & $1,1 \cdot 10^{-8}$ & & $8,2 \cdot 10^{-9}$ & $1,3 \cdot 10^{-7}$ \\
\hline
\end{tabular}


Tab. A-45: Durchfluß-Experimente mit Illit $H$-35 Versuch VII(3).

\section{VII(3)}

Illit H-35

$\varnothing=0,63-2 \mu \mathrm{m}$

Einwaage Mineral [g]: 0,7204

Einwaage Lösung [g]: 249,8

Lösung: $\mathbf{H}_{2} \mathrm{SO}_{4}, \mathbf{p H}=\mathbf{3}$

\begin{tabular}{|c|c|c|c|c|c|c|c|c|}
\hline & & & & $\mu \mathrm{mol} / \mathrm{l}$ & & & & \\
\hline Probe & Tage & $\mathrm{g} / \mathrm{h}$ & pH & $\mathbf{A l}$ & $\mathbf{F e}$ & $\mathbf{K}$ & Mg & Si \\
\hline$\overline{\mid V I I(3)-1 ~}$ & $\overline{7,50}$ & 2,87 & $\overline{3,1}$ & 1,4 & 0,30 & 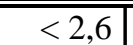 & 1,0 & 9,0 \\
\hline VII(3)-2 & 12,53 & 2,77 & 3,0 & 2,2 & 0,35 & $<2,6$ & 0,84 & 8,1 \\
\hline VII(3)-3 & 19,58 & 2,69 & 3,0 & 2,8 & 0,69 & 3,3 & 1,5 & 9,2 \\
\hline VII(3)-4 & 26,65 & 2,57 & 3,0 & 3,7 & $<0,18$ & $<2,6$ & 3,7 & 10 \\
\hline VII(3)-5 & 33,55 & 2,55 & 3,0 & 4,1 & $<0,18$ & $<2,6$ & 3,8 & 11 \\
\hline VII(3)-6 & 40,57 & 2,53 & 3,0 & 4,5 & $<0,18$ & $<2,6$ & 3,9 & 11 \\
\hline VII(3)-7 & 47,54 & 2,59 & 2,9 & 4,8 & $<0,18$ & $<2,6$ & 3,8 & 11 \\
\hline VII(3)-8 & 112,601 & 2,68 & 3,0 & 2,5 & 0,66 & 2,7 & 2,0 & 8,7 \\
\hline VII(3)-9 & 121,639 & 2,86 & 2,9 & 3,1 & 0,78 & 2,6 & 2,0 & 8,7 \\
\hline VII(3)-10 & 125,604 & 2,70 & 2,9 & 3,2 & 0,76 & 2,7 & 1,9 & 8,4 \\
\hline \begin{tabular}{|l|}
$\mathrm{VII}(3)-11$ \\
\end{tabular} & 131,670 & 2,71 & 2,9 & 3,1 & 0,75 & 2,7 & 1,9 & 8,4 \\
\hline \begin{tabular}{|l|}
$\mathrm{VII}(3)-12$ \\
\end{tabular} & 138,674 & 2,38 & 2,9 & 3,4 & 0,83 & 3,1 & 2,0 & 8,7 \\
\hline VII(3)-13 & 159,545 & 2,66 & 2,8 & 3,5 & 0,81 & 3,0 & 2,0 & 9,3 \\
\hline VII(3)-14 & 166,615 & 2,57 & 2,9 & 3,7 & 0,90 & 3,0 & 1,9 & 9,3 \\
\hline \multirow{2}{*}{\multicolumn{2}{|c|}{$\begin{array}{l}\text { Rate }[\mathrm{mol} /(\mathrm{g} \bullet \mathrm{h})] \\
\end{array}$}} & & & & & & & \\
\hline & & & & $1,2 \cdot 10^{-8}$ & $3,0 \cdot 10^{-9}$ & $1,0 \bullet 10^{-8}$ & $7,2 \cdot 10^{-9}$ & $3,2 \cdot 10^{-8}$ \\
\hline
\end{tabular}




\section{Illit OECD}

Tab. A-46: Batch-Experimente mit Illit OECD Versuche 19 und 22.

19

Illit OECD

$\varnothing=<0,63 \mu \mathrm{m}$

Einwaage Mineral [g]: 0,1505

Einwaage Lösung [g]: 100,0064

Lösung: $\mathrm{H}_{2} \mathrm{SO}_{4}, \mathbf{p H}=\mathbf{1}$

\begin{tabular}{|c|c|c|c|c|c|c|c|}
\hline \multirow[b]{2}{*}{ Probe } & \multirow[b]{2}{*}{ Tage } & \multirow[b]{2}{*}{$\mathbf{p H}$} & \multicolumn{5}{|l|}{$\mu \mathrm{mol} / 1$} \\
\hline & & & Al & $\mathbf{F e}$ & $\mathbf{K}$ & $\mathbf{M g}$ & $\mathbf{S} \mathbf{i}$ \\
\hline $19-1$ & 33,92 & $\overline{1,0}$ & 938 & 271 & $\overline{590}$ & 416 & 1.809 \\
\hline $19-2$ & 74,99 & 1,1 & 1.885 & 665 & 866 & 782 & 3.316 \\
\hline $19-3$ & 116,85 & 0,9 & 1.972 & 725 & 1.027 & 934 & 3.499 \\
\hline $19-4$ & 348,98 & 0,8 & 2.884 & 1.001 & 1.118 & 1.079 & 3.685 \\
\hline $19-5$ & 471,90 & 1,0 & 3.016 & 1.313 & 1.562 & 1.497 & 3.655 \\
\hline \multicolumn{3}{|c|}{ Rate $[\mathrm{mol} /(\mathrm{g} \bullet \mathrm{h})] 34-75$ Tage } & $6,4 \cdot 10^{-7}$ & $2,7 \cdot 10^{-7}$ & $1,9 \cdot 10^{-7}$ & $2,5 \cdot 10^{-7}$ & $1,0 \cdot 10^{-6}$ \\
\hline \multicolumn{3}{|c|}{ Rate $[\mathrm{mol} /(\mathrm{g} \bullet \mathrm{h})] 75-132$ Tage } & $5,8 \cdot 10^{-8}$ & $4,0 \cdot 10^{-8}$ & $1,7 \cdot 10^{-7}$ & $1,0 \bullet 10^{-7}$ & $1,2 \cdot 10^{-7}$ \\
\hline \multicolumn{3}{|c|}{ Rate $[\mathrm{mol} /(\mathrm{g} \bullet \mathrm{h})] 132-472$ Tage } & $8,5 \cdot 10^{-8}$ & $4,4 \cdot 10^{-8}$ & $3,8 \cdot 10^{-8}$ & $4,0 \cdot 10^{-8}$ & $1,3 \cdot 10^{-8}$ \\
\hline
\end{tabular}

\section{2}

\section{Illit OECD}

$\varnothing=<0,63 \mu \mathrm{m}$

Einwaage Mineral [g]: 0,1510

Einwaage Lösung [g]: 100,0022

Lösung: $\mathrm{H}_{2} \mathrm{SO}_{4}, \mathrm{pH}=\mathbf{2}$

\begin{tabular}{|c|c|c|c|c|c|c|c|}
\hline \multirow[b]{2}{*}{ Probe } & \multirow[b]{2}{*}{ Tage } & \multirow[b]{2}{*}{$\mathbf{p H}$} & \multicolumn{5}{|l|}{$\mu \mathrm{mol} / 1$} \\
\hline & & & Al & $\mathbf{F e}$ & $\mathbf{K}$ & Mg & $\mathbf{S}$ i \\
\hline $22-1$ & 33,98 & 1,7 & 172 & 23 & 141 & 136 & 459 \\
\hline $22-2$ & 75,00 & 2,0 & 329 & 59 & 137 & 188 & 758 \\
\hline $22-3$ & 116,88 & 1,9 & 371 & 83 & 182 & 248 & 868 \\
\hline $22-4$ & 131,81 & 1,9 & 412 & 100 & 200 & 253 & 1.083 \\
\hline $22-5$ & 312,84 & 1,9 & 720 & 173 & 298 & 344 & 1.506 \\
\hline $22-6$ & 349,71 & 1,9 & 804 & 215 & 322 & 403 & 1.983 \\
\hline $22-7$ & 471,90 & 1,9 & 941 & 362 & 535 & 638 & 2.372 \\
\hline \multicolumn{2}{|c|}{ Rate $[\mathrm{mol} /(\mathrm{g} \bullet \mathrm{h})]$} & & $4,7 \cdot 10^{-8}$ & $1,9 \cdot 10^{-8}$ & $2,3 \cdot 10^{-8}$ & $2,7 \cdot 10^{-8}$ & $1,1 \cdot 10^{-7}$ \\
\hline
\end{tabular}


Tab. A-47: Batch-Experimente mit Illit OECD Versuche 29 und 34.

29

Illit OECD

$\varnothing=<0,63 \mu \mathrm{m}$

Einwaage Mineral [g]: 0,1500

Einwaage Lösung [g]: 100,0026

Lösung: $\mathrm{H}_{2} \mathrm{SO}_{4}, \mathbf{p H}=\mathbf{3}$

\begin{tabular}{|c|c|c|c|c|c|c|c|}
\hline \multirow[b]{2}{*}{ Probe } & \multirow[b]{2}{*}{ Tage } & \multirow[b]{2}{*}{$\mathbf{p H}$} & \multicolumn{5}{|l|}{$\mu \mathrm{mol} / 1$} \\
\hline & & & A l & $\mathbf{F e}$ & $\mathbf{K}$ & $\mathbf{M g}$ & $\mathbf{S} \mathbf{i}$ \\
\hline $29-1$ & 2,22 & 4,0 & $\overline{15}$ & $<0,18$ & 16 & 22 & 38 \\
\hline $29-2$ & 17,13 & 4,0 & 14 & $<0,18$ & 6,4 & 23 & 79 \\
\hline $29-3$ & 198,16 & 3,4 & 10 & $<0,18$ & 14 & 24 & 112 \\
\hline $29-4$ & 234,28 & 4,2 & 12 & 0,50 & 16 & 27 & 129 \\
\hline $29-5$ & 357,22 & 4,2 & 10 & 0,39 & 24 & 38 & 138 \\
\hline \multicolumn{2}{|c|}{ Rate $[\mathrm{mol} /(\mathrm{g} \bullet \mathrm{h})]$} & & $-3,8 \cdot 10^{-10}$ & $-2,5 \cdot 10^{-11}$ & $8,8 \cdot 10^{-10}$ & $1,0 \cdot 10^{-9}$ & $7,0 \cdot 10^{-9}$ \\
\hline
\end{tabular}

29

Illit OECD

$\varnothing=<0,63 \mu \mathrm{m}$

Einwaage Mineral [g]: 0,1500

Einwaage Lösung [g]: 100,0026

Lösung: $\mathrm{H}_{2} \mathrm{SO}_{4}, \mathbf{p H}=3$

\begin{tabular}{|c|c|c|c|c|c|c|c|}
\hline \multirow[b]{2}{*}{ Probe } & \multirow[b]{2}{*}{ Tage } & \multirow[b]{2}{*}{$\mathbf{p H}$} & \multicolumn{5}{|l|}{$\mu \mathrm{mol} / 1$} \\
\hline & & & Al & $\mathbf{F e}$ & $\mathbf{K}$ & Mg & $\mathbf{S} \mathbf{i}$ \\
\hline $29-1$ & 2,22 & 4,0 & 15 & $<0,18$ & 16 & 22 & 38 \\
\hline $29-2$ & 17,13 & 4,0 & 14 & $<0,18$ & 14 & 23 & 79 \\
\hline $29-3$ & 198,16 & 3,4 & 10 & $<0,18$ & 14 & 24 & 112 \\
\hline $29-4$ & 234,28 & 4,2 & 12 & 0,50 & 16 & 27 & 129 \\
\hline $29-5$ & 357,22 & 4,2 & 10 & 0,39 & 24 & 38 & 138 \\
\hline \multicolumn{2}{|c|}{ Rate $[\mathrm{mol} /(\mathrm{g} \bullet \mathrm{h})]$} & & $-3,8 \cdot 10^{-10}$ & $-2,5 \cdot 10^{-11}$ & $5,4 \cdot 10^{-10}$ & $1,0 \cdot 10^{-9}$ & $7,0 \cdot 10^{-9}$ \\
\hline
\end{tabular}


Tab. A-48: Batch-Experimente mit Illit OECD Versuche 35 und 36.

\section{5}

\section{Illit OECD}

$\emptyset=<0,63 \mu \mathrm{m}$

Einwaage Mineral [g]: 0,1503

Einwaage Lösung [g]: 100,0087

Lösung: $\mathbf{H N O}_{3}, \mathbf{p H}=\mathbf{2}$

\begin{tabular}{|c|c|c|c|c|c|c|c|}
\hline \multirow[b]{2}{*}{ Probe } & \multirow[b]{2}{*}{ Tage } & \multirow[b]{2}{*}{$\mathbf{p H}$} & \multicolumn{5}{|l|}{$\mu \mathrm{mol} / 1$} \\
\hline & & & Al & Fe & $\mathbf{K}$ & Mg & $\mathbf{S} \mathbf{i}$ \\
\hline $35-1$ & 2,25 & $\overline{2,0}$ & 32 & 0,55 & 30 & $\overline{50}$ & 110 \\
\hline $35-2$ & 17,15 & 2,1 & 63 & 1,5 & 37 & 72 & 263 \\
\hline $35-3$ & 198,18 & 2,1 & 217 & 6,4 & 97 & 120 & 629 \\
\hline $35-4$ & 235,03 & 2,2 & 267 & 11 & 109 & 153 & 805 \\
\hline $35-5$ & 286,04 & 2,0 & 271 & 18 & 150 & 186 & 754 \\
\hline $35-6$ & 357,24 & 2,0 & 314 & 17 & 163 & 237 & 945 \\
\hline \multicolumn{2}{|c|}{ Rate $[\mathrm{mol} /(\mathrm{g} \bullet \mathrm{h})]$} & & $2,2 \cdot 10^{-8}$ & $1,4 \cdot 10^{-9}$ & $1,1 \cdot 10^{-8}$ & $1,3 \cdot 10^{-8}$ & $6,2 \cdot 10^{-8}$ \\
\hline
\end{tabular}

\section{6}

\section{Illit OECD}

$\emptyset=<0,63 \mu \mathrm{m}$

Einwaage Mineral [g]: 0,1501

Einwaage Lösung [g]: 100,0018

Lösung: $\mathrm{HNO}_{3}, \mathbf{p H}=\mathbf{3}$

\begin{tabular}{|c|c|c|c|c|c|c|c|}
\hline \multirow[b]{2}{*}{ Probe } & \multirow[b]{2}{*}{ Tage } & \multirow[b]{2}{*}{ pH } & \multicolumn{5}{|l|}{$\mu \mathrm{mol} / 1$} \\
\hline & & & Al & $\mathbf{F e}$ & $\mathbf{K}$ & Mg & $\mathbf{S} \mathbf{i}$ \\
\hline $36-1$ & 2,25 & 3,8 & 14 & $<0,18$ & 16 & 22 & 38 \\
\hline $36-2$ & 17,15 & 4,3 & 11 & $<0,18$ & 11 & 20 & 74 \\
\hline $36-3$ & 159,41 & 3,3 & 8,3 & $<0,18$ & 11 & 15 & 88 \\
\hline $36-4$ & 235,03 & 4,8 & 7,6 & 0,46 & 10 & 18 & 115 \\
\hline $36-5$ & 357,24 & 3,7 & 15 & 0,34 & 24 & 38 & 144 \\
\hline \multicolumn{2}{|c|}{ Rate $[\mathrm{mol} /(\mathrm{g} \bullet \mathrm{h})]$} & & $2,1 \cdot 10^{-11}$ & $-2,7 \cdot 10^{-11}$ & $5,6 \cdot 10^{-10}$ & $9,8 \cdot 10^{-10}$ & $7,1 \cdot 10^{-9}$ \\
\hline
\end{tabular}


Tab. A-49: Batch-Experimente mit Illit OECD Versuche 43 und 48. IN kenzeichnet die InputLösung.

43

Illit OECD

$\varnothing=<0,63 \mu \mathrm{m}$

Einwaage Mineral [g]: 0,1490

Einwaage Lösung [g]: 100,0057

Lösung: $\mathrm{C}_{6} \mathrm{H}_{8} \mathrm{O}_{7} \cdot \mathrm{H}_{2} \mathrm{O}, \mathrm{M}=\mathbf{0 , 0 1}$

\begin{tabular}{|c|c|c|c|c|c|c|c|}
\hline & & & \multicolumn{5}{|l|}{$\mu \mathrm{mol} / 1$} \\
\hline $\begin{array}{l}\text { Probe } \\
\end{array}$ & Tage & $\mathrm{pH}$ & $\overline{\mathrm{Al}}$ & $\mathbf{F e}$ & $\overline{\mathbf{K}}$ & $\mathbf{M g}$ & $\overline{\mathbf{S i}}$ \\
\hline $43-1$ & 2,25 & 2,6 & 56 & 3,8 & 25 & 38 & 99 \\
\hline $43-2$ & 17,14 & 2,6 & 97 & 9,7 & 33 & 58 & 265 \\
\hline $43-3$ & 198,17 & 2,9 & 229 & 37 & 90 & 89 & 601 \\
\hline $43-4$ & 235,11 & 3,2 & 312 & 64 & 100 & 145 & 853 \\
\hline $43-5$ & 288,20 & 4,9 & 278 & 57 & 124 & 158 & 734 \\
\hline $43-6$ & 357,24 & 5,4 & 47 & 27 & 104 & 167 & 732 \\
\hline \multirow{2}{*}{\multicolumn{2}{|c|}{ Rate $[\mathrm{mol} /(\mathrm{g} \bullet \mathrm{h})]$}} & & & & & & \\
\hline & & & $2,7 \cdot 10^{-8}$ & $6,2 \cdot 10^{-9}$ & $8,9 \cdot 10^{-9}$ & $9,9 \cdot 10^{-9}$ & $7,6 \cdot 10^{-8}$ \\
\hline
\end{tabular}

48

Illit OECD

$\varnothing=<0,63 \mu \mathrm{m}$

Einwaage Mineral [g]: 0,0502

Einwaage Lösung [g]: 30,0351

Lösung: abgepreßte Porenlösung Solling 0-15 cm Tiefe

pH $=3,5$

DOC $=39,15 \mathrm{mg} / \mathrm{l}$

\begin{tabular}{|c|r|r|r|r|r|r|r|}
\hline & \multicolumn{7}{|c|}{$\mu \mathrm{mol} / 1$} \\
Probe & \multicolumn{1}{c|}{ Tage } & \multicolumn{1}{c|}{$\mathbf{p H}$} & \multicolumn{1}{c|}{ A l } & \multicolumn{1}{c|}{ F } & \multicolumn{1}{c|}{ K } & M g & \multicolumn{1}{c|}{ S i } \\
\hline \hline IN & 0 & 3,5 & 20 & 6,3 & 58 & 59 & 362 \\
\hline $48-1$ & 24,99 & 5,6 & 5,8 & 0,58 & 55 & 47 & 401 \\
\hline $48-2$ & 62,01 & 5,7 & 3,7 & 0,70 & 61 & 50 & 445 \\
\hline $48-4$ & 184,03 & 5,9 & 2,9 & 1,2 & 76 & 63 & 445 \\
\hline
\end{tabular}


Tab. A-50: Durchfluß-Experimente mit Illit OECD Versuch XII(1).

XII(1)

\section{Illit OECD}

$\varnothing=<0,63 \mu \mathrm{m}$

Einwaage Mineral [g]: 0,4804

Einwaage Lösung [g]: 90,0

Lösung: $\mathrm{H}_{2} \mathrm{SO}_{4}, \mathbf{p H}=1$

\begin{tabular}{|c|c|c|c|c|c|c|c|c|}
\hline & & & & $\mu \mathrm{mol} / 1$ & & & & \\
\hline Probe & Tage & g/h & $\mathbf{p H}$ & Al & $\mathbf{F e}$ & $\mathbf{K}$ & $\mathrm{Mg}$ & Si \\
\hline XII(1)-1 & $\overline{5,10}$ & $\overline{0,63}$ & $\overline{1,4}$ & $\overline{4472}$ & 63 & 218 & 285 & 1.134 \\
\hline XII(1)-2 & 7,07 & 0,95 & 1,4 & 382 & 64 & 176 & 235 & 905 \\
\hline XII(1)-3 & 8,18 & 0,68 & 1,4 & 384 & 71 & 181 & 237 & 928 \\
\hline XII(1)-4 & 13,36 & 0,56 & 1,6 & 158 & 47 & 76 & 92 & 354 \\
\hline XII(1)-5 & 14,09 & 0,54 & 1,3 & 79 & 28 & 41 & 50 & 190 \\
\hline XII(1)-6 & 45,98 & 2,52 & 1,1 & 29 & 14 & 15 & 14 & 70 \\
\hline XII(1)-7 & 48,00 & 2,14 & 1,1 & 25 & 10 & 13 & 11 & 55 \\
\hline XII(1)-8 & 50,87 & 2,88 & 1,1 & 24 & 10 & 11 & 10 & 61 \\
\hline XII(1)-9 & 54,07 & 2,40 & 1,1 & 23 & 9,6 & 11 & 9,5 & 58 \\
\hline XII(1)-10 & 58,08 & 2,32 & 1,1 & 15 & 6,1 & 7,6 & 6,2 & 38 \\
\hline XII(1)-11 & 62,00 & 2,35 & 1,1 & 13 & 5,0 & 6,2 & 5,0 & 31 \\
\hline XII(1)-12 & 64,99 & 2,38 & 1,1 & 12 & 4,6 & 5,8 & 4,6 & 30 \\
\hline XII(1)-13 & 68,03 & 2,34 & 1,1 & 14 & 5,3 & 6,6 & 5,2 & 35 \\
\hline XII(1)-14 & 72,21 & 2,09 & 1,1 & 11 & 3,9 & 5,4 & 3,9 & 27 \\
\hline XII(1)-15 & 75,00 & 2,69 & 1,1 & 17 & 5,8 & 7,4 & 5,7 & 41 \\
\hline XII(1)-16 & 83,01 & 2,44 & 1,1 & 25 & 7,2 & 8,9 & 5,8 & 47 \\
\hline \multicolumn{2}{|c|}{ Rate $[\mathrm{mol} /(\mathrm{g} \bullet \mathrm{h})]$} & & & $7,6 \cdot 10^{-8}$ & $2,7 \cdot 10^{-8}$ & $3,4 \cdot 10^{-8}$ & $2,6 \cdot 10^{-8}$ & $1,8 \cdot 10^{-7}$ \\
\hline
\end{tabular}


Tab. A-51: Durchfluß-Experimente mit Illit OECD Versuch XII(2).

\section{$\operatorname{XII}(2)$}

\section{Illit OECD}

$\emptyset=<0,63 \mu \mathrm{m}$

Einwaage Mineral [g]: 0,4804

Einwaage Lösung [g]: 90,0

Lösung: $\mathbf{H}_{2} \mathrm{SO}_{4}, \mathbf{p H}=\mathbf{2}$

\begin{tabular}{|c|c|c|c|c|c|c|c|c|}
\hline & & & & $\mu \mathrm{mol} / \mathrm{l}$ & & & & \\
\hline Probe & Tage & g/h & pH & Al & $\mathbf{F e}$ & $\mathbf{K}$ & Mg & $\mathbf{S i}$ \\
\hline XII(2)-1 & 17,06 & 2,44 & 2,0 & 11 & 2,6 & 4,1 & $<0,82$ & 16 \\
\hline XII(2)-2 & 20,04 & 2,42 & 2,1 & 2,8 & 1,3 & $<2,6$ & $<0,82$ & 4,6 \\
\hline XII(2)-3 & 35,95 & 2,78 & 2,1 & 1,7 & 0,34 & $<2,6$ & $<0,82$ & 3,9 \\
\hline XII(2)-4 & 41,95 & 2,73 & 1,6 & 3,3 & 0,64 & $<2,6$ & $<0,82$ & 6,0 \\
\hline XII(2)-5 & 44,89 & 2,86 & 1,7 & 2,2 & 0,42 & $<2,6$ & $<0,82$ & 4,4 \\
\hline XII(2)-6 & 54,95 & 2,62 & 1,9 & 1,6 & 0,27 & $<2,6$ & $<0,82$ & 4,0 \\
\hline XII(2)-7 & 57,08 & 1,90 & 1,9 & 1,7 & 0,39 & $<2,6$ & $<0,82$ & 4,2 \\
\hline XII(2)-8 & 58,97 & 2,69 & 1,9 & 1,6 & 0,25 & $<2,6$ & $<0,82$ & 3,7 \\
\hline XII(2)-9 & 61,93 & 2,65 & 1,9 & 1,5 & 0,22 & $<2,6$ & $<0,82$ & 3,3 \\
\hline XII(2)-10 & 68,99 & 2,66 & 2,0 & 1,3 & 0,20 & $<2,6$ & $<0,82$ & 1,0 \\
\hline XII(2)-11 & 75,99 & 2,53 & 2,0 & 1,2 & $<0,18$ & $<2,6$ & $<0,82$ & 0,88 \\
\hline XII(2)-12 & 78,03 & 2,45 & 2,0 & 1,2 & $<0,18$ & $<2,6$ & $<0,82$ & 0,94 \\
\hline XII(2)-13 & 78,97 & 2,66 & 2,0 & 1,1 & $<0,18$ & $<2,6$ & $<0,82$ & 0,95 \\
\hline XII(2)-14 & 84,92 & 2,68 & 2,0 & 1,3 & $<0,18$ & $<2,6$ & $<0,82$ & 1,1 \\
\hline XII(2)-15 & 90,88 & 2,74 & 2,0 & 1,3 & $<0,18$ & $<2,6$ & $<0,82$ & 1,2 \\
\hline XII(2)-16 & 92,86 & 2,74 & 2,0 & 1,2 & $<0,18$ & $<2,6$ & $<0,82$ & 1,1 \\
\hline XII(2)-17 & 96,95 & 2,69 & 2,0 & 1,2 & $<0,18$ & $<2,6$ & $<0,82$ & 1,0 \\
\hline XII(2)-18 & 99,01 & 2,69 & 2,0 & 1,5 & 0,22 & $<2,6$ & $<0,82$ & 1,3 \\
\hline XII(2)-19 & 103,91 & 2,73 & 2,0 & 1,4 & $<0,18$ & $<2,6$ & $<0,82$ & 1,4 \\
\hline XII(2)-20 & 105,97 & 2,77 & 1,9 & 1,3 & 0,21 & $<2,6$ & $<0,82$ & 4,4 \\
\hline XII(2)-21 & 107,89 & 2,75 & 1,9 & 1,3 & $<0,18$ & $<2,6$ & $<0,82$ & 3,8 \\
\hline XII(2)-22 & 112,94 & 2,66 & 1,9 & 1,2 & 0,25 & $<2,6$ & $<0,82$ & 3,8 \\
\hline XII(2)-23 & 114,99 & 2,62 & 1,9 & 1,2 & $<0,18$ & $<2,6$ & $<0,82$ & 3,9 \\
\hline XII(2)-24 & 117,96 & 2,74 & 1,9 & 1,3 & 0,24 & $<2,6$ & $<0,82$ & 3,7 \\
\hline XII(2)-25 & 119,89 & 2,85 & 1,9 & 1,3 & 0,19 & $<2,6$ & $<0,82$ & 3,8 \\
\hline XII(2)-26 & 122,03 & 2,26 & 1,9 & 1,4 & $<0,18$ & $<2,6$ & $<0,82$ & 3,7 \\
\hline XII(2)-27 & 125,90 & 2,31 & 1,8 & 2,2 & 0,33 & $<2,6$ & $<0,82$ & 4,9 \\
\hline XII(2)-28 & 128,01 & 2,85 & 1,9 & 1,5 & 0,23 & $<2,6$ & $<0,82$ & 4,1 \\
\hline XII(2)-29 & 131,97 & 2,91 & 1,9 & 1,5 & 0,21 & $<2,6$ & $<0,82$ & 4,0 \\
\hline XII(2)-30 & 135,05 & 2,75 & 1,8 & 1,6 & 0,22 & $<2,6$ & $<0,82$ & 3,9 \\
\hline XII(2)-31 & 138,92 & 2,92 & 1,9 & 1,7 & 0,27 & $<2,6$ & $<0,82$ & 4,3 \\
\hline XII(2)-32 & 141,13 & 2,61 & 1,9 & 2,9 & 0,54 & $<2,6$ & $<0,82$ & 6,1 \\
\hline XII(2)-33 & 143,00 & 2,69 & 1,9 & 1,6 & 0,22 & $<2,6$ & $<0,82$ & 4,0 \\
\hline XII(2)-34 & 146,92 & 2,74 & 1,8 & 1,5 & 0,20 & $<2,6$ & $<0,82$ & 3,8 \\
\hline XII(2)-35 & 150,00 & 2,81 & 1,8 & 1,6 & 0,21 & $<2,6$ & $<0,82$ & 4,0 \\
\hline XII(2)-36 & 152,89 & 2,81 & 1,9 & 1,5 & 0,22 & $<2,6$ & $<0,82$ & 4,0 \\
\hline \multicolumn{2}{|c|}{ Rate $[\mathrm{mol} /(\mathrm{g} \bullet \mathrm{h})]$} & & & $1,0 \cdot 10^{-8}$ & $1,5 \cdot 10^{-9}$ & & & $2,5 \cdot 10^{-8}$ \\
\hline
\end{tabular}




\section{Talk}

Tab. A-52: Batch-Experimente mit Talk Versuche 20 und 23.

20

Talk OECD

$\varnothing=2-63 \mu \mathrm{m}$

Einwaage Mineral [g]: 0,1506

Einwaage Lösung [g]: 100,0040

Lösung: $\mathbf{H}_{2} \mathbf{S O}_{4}, \mathbf{p H}=\mathbf{1}$

\begin{tabular}{|c|c|c|c|c|c|c|}
\hline \multirow[b]{2}{*}{ Probe } & \multirow[b]{2}{*}{ Tage } & \multirow[b]{2}{*}{ pH } & \multicolumn{4}{|l|}{$\mu \mathrm{mol} / 1$} \\
\hline & & & Al & $\mathbf{F e}$ & Mg & $\mathbf{S}$ i \\
\hline $20-1$ & 33,92 & 0,9 & 19 & 9,6 & 260 & 87 \\
\hline $20-2$ & 74,99 & 1,1 & 35 & 12 & 331 & 155 \\
\hline $20-3$ & 116,85 & 0,9 & 38 & 13 & 403 & 182 \\
\hline $20-4$ & 131,80 & 0,9 & 43 & 14 & 418 & 233 \\
\hline $20-6$ & 312,83 & 0,8 & 71 & 16 & 577 & 467 \\
\hline $20-7$ & 349,71 & 1,1 & 78 & 21 & 644 & 593 \\
\hline $20-8$ & 471,90 & 0,9 & 94 & 31 & 1.106 & 816 \\
\hline \multicolumn{2}{|c|}{ Rate $[\mathrm{mol} /(\mathrm{g} \bullet \mathrm{h})]$} & & $4,5 \cdot 10^{-9}$ & $1,1 \cdot 10^{-9}$ & $4,5 \cdot 10^{-8}$ & $4,5 \cdot 10^{-8}$ \\
\hline
\end{tabular}

\section{3}

Talk OECD

$\varnothing=2-63 \mu \mathrm{m}$

Einwaage Mineral [g]: 0,1510

Einwaage Lösung [g]: 100,0005

Lösung: $\mathrm{H}_{2} \mathrm{SO}_{4}, \mathbf{p H}=\mathbf{2}$

\begin{tabular}{|c|c|c|c|c|c|c|}
\hline \multirow[b]{2}{*}{ Probe } & \multirow[b]{2}{*}{ Tage } & \multirow[b]{2}{*}{ pH } & \multicolumn{4}{|l|}{$\mu \mathrm{mol} / 1$} \\
\hline & & & Al & $\mathbf{F e}$ & Mg & $\mathbf{S}$ i \\
\hline $23-1$ & 33,98 & $\overline{1,8}$ & $\overline{9,4}$ & 7,1 & 200 & 30 \\
\hline $23-2$ & 75,00 & 2,0 & 17 & 9,7 & 247 & 54 \\
\hline $23-3$ & 116,88 & 1,9 & 18 & 9,6 & 282 & 62 \\
\hline $23-4$ & 131,81 & 1,8 & 20 & 10 & 285 & 77 \\
\hline $23-5$ & 273,96 & 2,0 & 30 & 13 & 309 & 102 \\
\hline $23-6$ & 349,71 & 1,9 & 34 & 13 & 340 & 141 \\
\hline $23-7$ & 471,90 & 1,9 & 39 & 18 & 444 & 166 \\
\hline \multicolumn{2}{|c|}{ Rate $[\mathrm{mol} /(\mathrm{g} \bullet \mathrm{h})]$} & & $1,8 \cdot 10^{-9}$ & $5,9 \cdot 10^{-10}$ & $1,3 \cdot 10^{-8}$ & $8,3 \cdot 10^{-9}$ \\
\hline
\end{tabular}


Tab. A-53: Durchfluß-Experimente mit Talk Versuch XI.

XI

Talk OECD

$\emptyset=2-63 \mu \mathrm{m}$

Einwaage Mineral [g]: 0,4007

Einwaage Lösung [g]: 88,1

Lösung: $\mathrm{H}_{2} \mathrm{SO}_{4}, \mathbf{p H}=\mathbf{1}$

\begin{tabular}{|c|c|c|c|c|c|c|c|}
\hline & & & & $\mu \mathrm{mol} / 1$ & & & \\
\hline Probe & Tage & g/h & pH & Al & $\mathbf{F e}$ & $\mathrm{Mg}$ & $\mathbf{S i}$ \\
\hline XI-1 & 19,99 & 2,25 & 1,1 & 6,4 & 2,2 & 133 & 128 \\
\hline $\mathrm{XI}-2$ & 22,97 & 2,28 & 1,1 & 6,4 & 2,2 & 135 & 136 \\
\hline XI-3 & 26,02 & 2,23 & 1,1 & 7,1 & 2,5 & 149 & 162 \\
\hline XI-4 & 30,19 & 2,10 & 1,2 & 6,0 & 2,2 & 140 & 148 \\
\hline XI-5 & 32,99 & 2,22 & 1,2 & 6,1 & 4,6 & 139 & 147 \\
\hline XI-6 & 40,99 & 2,41 & 1,1 & 11 & 2,3 & 162 & 162 \\
\hline XI-7 & 58,05 & 1,76 & 1,0 & 20 & 1,5 & 66 & 92 \\
\hline XI-8 & 61,03 & 1,85 & 1,1 & 9,7 & 0,77 & 35 & 46 \\
\hline XI-9 & 76,95 & 3,00 & 1,1 & 13 & 0,72 & 24 & 49 \\
\hline XI-10 & 82,94 & 2,29 & 1,1 & 7,5 & 0,32 & 8,0 & 23 \\
\hline XI-11 & 85,89 & 2,64 & 1,1 & 7,3 & 0,42 & 5,4 & 22 \\
\hline XI-12 & 95,95 & 2,52 & 1,0 & 6,8 & 0,32 & 5,2 & 24 \\
\hline XI-13 & 98,07 & 1,91 & 1,0 & 6,5 & 0,38 & 4,5 & 24 \\
\hline XI-14 & 99,96 & 2,58 & 1,0 & 6,2 & 0,30 & 4,2 & 24 \\
\hline $\mathrm{XI}-15$ & 102,92 & 2,56 & 1,0 & 5,3 & 0,26 & 3,4 & 21 \\
\hline XI-16 & 104,94 & 2,42 & 1,1 & 4,9 & 0,26 & 4,0 & 16 \\
\hline XI-17 & 107,07 & 1,95 & 1,1 & 5,0 & 0,28 & 4,1 & 18 \\
\hline XI-18 & 109,98 & 2,51 & 1,1 & 4,6 & 0,27 & 5,6 & 17 \\
\hline XI-19 & 116,99 & 2,50 & 1,1 & 4,4 & 0,28 & 10 & 24 \\
\hline XI-20 & 119,02 & 2,48 & 1,1 & 4,5 & 0,27 & 7,9 & 21 \\
\hline $\mathrm{XI}-21$ & 119,96 & 2,58 & 1,1 & 4,9 & 0,29 & 7,7 & 18 \\
\hline $\mathrm{XI}-22$ & 125,92 & 2,60 & 1,1 & 5,2 & 0,29 & 9,6 & 23 \\
\hline $\mathrm{XI}-23$ & 127,91 & 2,85 & 1,1 & 5,8 & 0,35 & 10 & 22 \\
\hline $\mathrm{XI}-24$ & 131,87 & 2,69 & 1,1 & 6,2 & 0,31 & 9,1 & 25 \\
\hline $\mathrm{XI}-25$ & 133,85 & 2,70 & 1,1 & 6,2 & 0,32 & 9,1 & 23 \\
\hline $\mathrm{XI}-26$ & 137,95 & 2,62 & 1,1 & 5,4 & 0,30 & 9,1 & 23 \\
\hline $\mathrm{XI}-27$ & 140,00 & 2,61 & 1,1 & 5,3 & 0,30 & 8,4 & 24 \\
\hline $\mathrm{XI}-28$ & 144,90 & 2,68 & 1,1 & 5,3 & 0,28 & 9,0 & 25 \\
\hline \multicolumn{2}{|c|}{ Rate $[\mathrm{mol} /(\mathrm{g} \bullet \mathrm{h})]$} & & & $3,5 \cdot 10^{-8}$ & $2,0 \cdot 10^{-9}$ & $5,9 \cdot 10^{-8}$ & $1,5 \cdot 10^{-7}$ \\
\hline
\end{tabular}


Tab. A-54: Durchfluß-Experimente mit Talk Versuch XIII.

\section{XIII}

Talk OECD

$\emptyset=2-63 \mu \mathrm{m}$

Einwaage Mineral [g]: 0,3998

Einwaage Lösung [g]: 87,2

Lösung: $\mathbf{H N O}_{3}, \mathbf{p H}=\mathbf{1}$

\begin{tabular}{|c|c|c|c|c|c|c|c|}
\hline & & & & $\mu \mathrm{mol} / 1$ & & & \\
\hline Probe & Tage & g/h & $\mathbf{p H}$ & Al & $\mathbf{F e}$ & $\mathrm{Mg}$ & $\mathbf{S i}$ \\
\hline XIII-1 & 3,97 & 2,57 & $\overline{1,1}$ & 5,1 & 4,0 & 119 & 32 \\
\hline XIII-2 & 5,99 & 2,16 & 1,1 & 4,1 & 3,2 & 101 & 34 \\
\hline XIII-3 & 8,86 & 2,90 & 1,2 & 3,6 & 2,2 & 75 & 46 \\
\hline XIII-4 & 12,06 & 2,40 & 1,2 & 2,2 & 1,2 & 43 & 34 \\
\hline XIII-5 & 16,07 & 2,33 & 1,2 & 1,9 & 0,69 & 27 & 35 \\
\hline XIII-6 & 19,99 & 2,34 & 1,2 & 2,0 & 0,54 & 22 & 32 \\
\hline XIII-7 & 22,97 & 2,39 & 1,2 & 1,9 & 0,44 & 20 & 34 \\
\hline XIII-8 & 26,02 & 2,34 & 1,1 & 2,3 & 0,51 & 25 & 41 \\
\hline XIII-9 & 30,19 & 2,16 & 1,1 & 2,5 & 0,81 & 21 & 36 \\
\hline XIII-10 & 32,99 & 2,79 & 1,2 & 2,7 & 0,47 & 18 & 34 \\
\hline XIII-11 & 40,99 & 2,49 & 1,0 & 2,3 & 0,25 & 4,4 & 18 \\
\hline XIII-12 & 104,94 & 2,46 & 1,1 & 2,4 & 0,30 & 2,5 & 28 \\
\hline XIII-13 & 107,07 & 2,02 & 1,1 & 2,9 & 0,44 & 3,5 & 26 \\
\hline XIII-14 & 109,98 & 2,59 & 1,1 & 2,4 & 0,29 & 2,6 & 21 \\
\hline XIII-15 & 116,99 & 2,49 & 1,1 & 2,4 & 0,26 & 2,0 & 21 \\
\hline XIII-16 & 119,02 & 2,41 & 1,1 & 2,4 & 0,28 & 1,7 & 21 \\
\hline XIII-17 & 119,96 & 2,59 & 1,1 & 2,6 & 0,27 & 1,7 & 21 \\
\hline XIII-18 & 125,92 & 2,60 & 1,1 & 2,6 & 0,24 & 1,6 & 20 \\
\hline XIII-19 & 127,91 & 2,78 & 1,1 & 3,7 & 0,35 & 2,1 & 24 \\
\hline XIII-20 & 131,87 & 2,69 & 1,1 & 2,5 & 0,28 & 1,6 & 19 \\
\hline XIII-21 & 133,85 & 2,70 & 1,1 & 3,3 & 0,28 & 1,8 & 21 \\
\hline XIII-22 & 140,00 & 2,63 & 1,1 & 2,7 & 0,32 & 2,3 & 20 \\
\hline XIII-23 & 144,90 & 2,70 & 1,1 & 4,5 & 0,55 & 7,5 & 25 \\
\hline XIII-24 & 146,96 & 2,74 & 1,1 & 3,0 & 0,28 & 1,7 & 20 \\
\hline XIII-25 & 148,88 & 2,64 & 1,1 & 2,9 & 0,26 & 1,7 & 19 \\
\hline XIII-26 & 153,93 & 2,59 & 1,1 & 2,5 & 0,29 & 1,4 & 17 \\
\hline XIII-27 & 155,99 & 2,61 & 1,1 & 2,4 & 0,33 & 1,2 & 17 \\
\hline XIII-28 & 158,95 & 2,76 & 1,0 & 2,7 & 0,22 & 1,3 & 17 \\
\hline XIII-29 & 160,88 & 2,81 & 1,0 & 2,7 & 0,21 & 1,2 & 17 \\
\hline XIII-30 & 163,02 & 2,28 & 1,0 & 2,8 & 0,22 & 1,3 & 17 \\
\hline XIII-31 & 166,90 & 2,84 & 1,0 & 3,6 & 0,28 & 3,4 & 19 \\
\hline XIII-32 & 169,00 & 2,70 & 1,0 & 3,4 & 0,28 & 2,4 & 18 \\
\hline XIII-33 & 172,96 & 2,84 & 1,0 & 3,1 & 0,32 & 1,8 & 16 \\
\hline XIII-34 & 176,05 & 2,72 & 1,0 & 2,8 & 0,28 & 1,6 & 15 \\
\hline XIII-35 & 179,92 & 2,91 & 1,0 & 3,3 & 0,26 & 1,6 & 16 \\
\hline XIII-36 & 182,13 & 2,59 & 1,0 & 3,6 & 0,49 & 1,7 & 17 \\
\hline XIII-37 & 183,99 & 2,68 & 1,0 & 2,8 & 0,25 & 1,4 & 15 \\
\hline XIII-38 & 187,92 & 2,70 & 1,0 & 2,9 & 0,25 & 1,3 & 14 \\
\hline XIII-39 & 191,00 & 2,78 & 1,0 & 3,1 & 0,22 & 1,3 & 15 \\
\hline XIII-40 & 193,89 & 2,75 & 1,0 & 3,2 & 0,24 & 1,4 & 15 \\
\hline \multicolumn{2}{|c|}{ Rate $[\mathrm{mol} /(\mathrm{g} \bullet \mathrm{h})]$} & & & $2,1 \cdot 10^{-8}$ & $2,0 \cdot 10^{-9}$ & $1,0 \cdot 10^{-8}$ & $1,1 \cdot 10^{-7}$ \\
\hline
\end{tabular}




\section{Danksagung}

Die Möglichkeit zur Bearbeitung dieses spannenden Themas verdanke ich Herrn Prof. Dr. Hartmut Heinrichs. Hierfür und für die gute Betreuung möchte ich mich herzlich bedanken.

Für die Übernahme des Korreferats bedanke ich mich bei PD Dr. Martin Dietzel.

Frau Dr. Annerose Heydemann verdanke ich, daß das Thema bis zum Schluß spannend blieb und selbst in manch stressiger Zeit seinen Reiz nicht verlor. Sie hat durch ihr nicht nachlassendes Interesse, ihre Diskussionsbereitschaft und viele Denkanstöße sehr zum gelingen der vorliegenden Arbeit beigetragen. Dafür vielen Dank.

Eine Vielzahl von Experimenten wäre ohne den findigen und oft „blitzschnellen” Einsatz von Reinhold Skrandies und Heinrich Herborg nicht möglich gewesen. Die von ihnen in liebevoller Kleinarbeit gebaute und regelmäßig gewartete Apparatur hat mir bei der Durchführung der vielen Versuche gute Dienste geleistet.

Für manch organisatorische Unterstützung im Laborbetrieb sei Angelika Reitz und Erwin Schiffczyk gedankt.

Für die fachkundige Betreuung am Feldemissions-REM und die Durchführung einiger AFMUntersuchungen im Institut für Nichtmetallische Werkstoffe in Clausthal-Zellerfeld danke ich Dr. Kirsten Techmer.

Bei Kathrin Brüschke bedanke ich mich für die Übernahme von einzelnen DOC-Messungen. Neben den genannten Mitarbeitern möchte ich mich auch bei allen nicht genannten „Insassen” des Geochemischen Instituts bedanken. Sie alle haben durch ein gutes Arbeitsklima zum gelingen der Arbeit beigetragen.

Für moralische Unterstützung, kritische Diskussionen und die Bereitschaft, sich mit dem Manuskript auseinanderzusetzen, möchte ich mich ganz herzlich bei Peter Barnekow bedanken.

Ein ganz besonderer Dank gilt auch Ute Volland und Dr. Christian Reutel für die unkomplizierte Verwaltung und die immer gewährte Unterstützung in verwaltungstechnischen Fragen.

Ohne die Finanzierung des Sonderforschungsbereiches (SFB 468) „Wechselwirkungen an geologischen Grenzflächen" durch die Deutsche Forschungsgemeinschaft hätte diese Arbeit im Teilprojekt A4 nicht entstehen können. Auch hierfür und für die Möglichkeit, verschiedene Tagungen und Workshops besuchen zu können, möchte ich mich herzlich bedanken. 


\section{Lebenslauf}

Name:

geb. am:

Staatsangehörigkeit:

Schulausbildung:

$1974-1984$

$1988-1991$

Berufsausbildung:

10/1984 - 06/1987

Hochschulausbildung:

10/1991 - 11/1996

15.10.1993

01.11 .1996

Berufstätigkeit:

07/1987 - 10/1991

seit 03/1997
Sabine Schlabach

17.03.1968 in Göttingen

deutsch

Schulausbildung in Göttingen

Erweiterter Sekundarabschluß I: Juli 1984

Abendgymnasium in Göttingen

Allgemeine Hochschulreife Mai 1991

Ausbildung zur Biologielaborantin an der

Niedersächsischen Forstlichen Versuchsanstalt in Göttingen

Studium der Mineralogie an der Georg-August-Universität Göttingen mit den Schwerpunkten Geochemie, Petrologie und Lagerstättenkunde

Vordiplomprüfungen

Thema der Diplomarbeit:

Konzentrationsänderungen natürlicher und anthropogener Inhaltsstoffe in Porenlösungen mit dem $\mathrm{pH}-$ Wert und der Tiefe auf versauerten Waldstandorten (Harz, Solling)

Betreuer: Prof. Dr. Hartmut Heinrichs

Diplomprüfungen

Technische Angestellte an der Niedersächsischen Forstlichen Versuchsanstalt in Göttingen - Abteilung Waldschutz (Mykologisches Labor) einschließlich Sachgebiet Luftbild

Wissenschaftliche Angestellte (Promotionsstelle) im Projekt „Wechselwirkungen zwischen Lösungen und Festkörpern in offenen Systemen und strömenden Medien" im SFB 468 / Geochemisches Institut der Georg-August-Universität Göttingen bei Prof. Dr. Hartmut Heinrichs 